Copyright

by

Thomas Rolf Mazur

2014 
The Dissertation Committee for Thomas Rolf Mazur

certifies that this is the approved version of the following dissertation:

\section{Magnetically Activated and Guided Isotope Separation}

Committee:

Mark G. Raizen, Supervisor

John Keto

John Markert

Greg Sitz

S.V. Sreenivasan 


\title{
Magnetically Activated and Guided Isotope Separation
}

by

Thomas Rolf Mazur, B.S.

\author{
DISSERTATION \\ Presented to the Faculty of the Graduate School of \\ The University of Texas at Austin \\ in Partial Fulfillment \\ of the Requirements \\ for the Degree of \\ DOCTOR OF PHILOSOPHY
}

THE UNIVERSITY OF TEXAS AT AUSTIN

August 2014 


\section{Acknowledgments}

I first would like to thank Mark Raizen. Speaking to Mark about his research initially motivated me to attend UT Austin. I owe Mark immense gratitude for him giving me the opportunity to do research under his supervision despite my limited background in experimental physics. When I began as a research assistant in Mark's lab in the fall of 2008, Mark and my colleagues at the time had recently pioneered two techniques - the "atomic coilgun" and single-photon cooling - that could in principle be applied in tandem toward the trapping and cooling of almost any atom in addition to many molecules. Being present as these ideas materialized and evolved was exciting and motivating. I thoroughly enjoyed working on applying these techniques toward the trapping and cooling of hydrogen atoms.

Mark's ideas evolved into real applications including lithography and isotope separation. What began as "single-photon atomic sorting" ultimately evolved into the topic of this dissertation: Magnetically Activated and Guided Isotope Separation. I am very grateful to Mark for the opportunity to contribute toward realizing MAGIS. Having been able to contribute to both basic research and a very imminent application, I think that my graduate experience has been truly unique. I admire Mark's efforts toward realizing MAGIS as a real tool for producing stable isotopes with diverse and beneficial applications, and I hope that this work will facilitate these efforts. Even while working on pressing research (like MAGIS), Mark afforded me a great deal of creative freedom that benefited me immensely in acquiring expertise in many areas.

Part of my unique experience involved working closely with Bruce Klappauf, a research scientist who co-proposed MAGIS with Mark and laid out the framework for our experiment using lithium. Bruce has very impressive experience and knowledge, and I learned a great deal while working on this experiment with him. I greatly appreciated his patience and willingness to explain things that were unclear to me (notably letting me contribute over his shoulder as he setup most of the laser system that we built). 
Bruce's Python simulations that facilitated the design for our apparatus were extremely impressive. Bruce was responsible for all of the simulations discussed in Chapter 2.

Prior to working on MAGIS, I worked toward trapping and cooling atomic hydrogen mainly with Adam Libson. I have immense respect for Adam's physical intuition: Adam explained many things to me in a simple manner. Due to my respect for him, Adam substantially influenced my way of thinking as a physicist. Building the quadrupole trap for interfacing to our coilgun was one of the most rewarding experiences for me as a graduate student. When we ran into problems, Adam repeatedly derived solutions; he has a penchant for staying calm and methodically tackling problems. I admire all of his work in Mark's lab, including helium slowing using the rotor apparatus and the early magnetic slowing of neon and molecular oxygen.

I also worked closely with Isaac Chavez on the hydrogen trapping project when I started as a graduate student. We started in graduate school at the same time, and took nearly all of the same courses. Isaac became a good friend as we struggled through the courses together. Isaac is a tremendously talented experimentalist who always finds simple solutions for realizing experimental goals. I was thrilled that Isaac returned to graduate school after his hiatus, and I am very confident that he will make large strides toward our interests in trapping and cooling hydrogen.

More recently, I worked together with Kevin Melin on different projects. Kevin made several key contributions toward realizing MAGIS. As an undergraduate, he constructed the prototypes for assembling the curved Halbach array that we used for magnetic separation. As a graduate student, Kevin has worked with Isaac toward hydrogen trapping. Kevin has impressive aptitude at automating aspects of an apparatus. I have admired his recent contributions toward the hydrogen efforts, notably incorporating various motorized assemblies for moving optics via software control. I always enjoy talking to Kevin about both experiments and other projects (like microcontrollers and FPGAs, among other things). Not achieving hydrogen trapping has been my greatest disappointment in graduate school, but I am confident that Isaac and Kevin will succeed where I did not! 
I worked with many other very bright and friendly people in Mark's lab. Simon Kheifets and Akarsh Simha studied Brownian motion at impressively short time-scales on an optical table immediately adjacent to the MAGIS apparatus. Kirsten Viering, David Medellin, and Jianyong Mo made valiant progress toward quantum computation using a demanding degenerate Fermi-gas apparatus adjacent to that table. More recently, Rodrigo Castillo-Garza, Jamie Gardner, and Erik Anciaux have made impressive progress toward high-resolution lithography using supersonic beams of metastable atoms. Charlotte Sanders - who also started at the same time as me and took many courses with Isaac and I - also contributed to efforts in the lab for some time. I think very highly of all of these colleagues, and I think of them all as friends. I particularly owe gratitude to Kirsten as she setup the Toptica laser system that we used heavily in this work.

Several students and post-docs paved the way for much of the ongoing research in the lab. Travis Bannerman and Rob Clark laid the framework for the optical system that will be used for detecting hydrogen. Ed Narevicius led the pioneering efforts for the atomic coilgun. Melissa Jerkins heavily simulated the precursor to MAGIS: single-photon atomic sorting. Tongcang Li built the awe-inspiring apparatus for cooling microspheres (and subsequently measuring Brownian motion at short time-scales). I very briefly overlapped with Gabriel Price and Hrishikesh Kelkar, both of whom I respected a great deal.

Many undergraduate students have worked in the lab while I have been a graduate student. I would particularly like to acknowledge Daniel Raimi-Zlatic. Daniel worked toward building an apparatus for in-situ cleaning of silicon wafers (in a vacuum chamber) followed by hydrogen passivation. Daniel made impressive progress for an undergraduate student: he successfully built an assembly for rapidly heating silicon wafers to over $900{ }^{\circ} \mathrm{C}$, and he also designed an atomic hydrogen source that should provide better performance than an earlier "cracker" that he inherited. I thoroughly enjoyed working together with Daniel. I worked briefly with several other very talented undergraduates notably including Daniel Ellsworth and Francisco Camargo. More recently, Tharon Morrison has continued working on Daniel's apparatus. 
The UT Physics Department has a collection of resources that have been instrumental toward my work. I particularly would like to thank the machine, cryogenics, and electronics shops. Jack Clifford, the supervisor for the student machine shop, assisted me with more projects than I can remember. I am indebted to him for helping me to both machine parts and improve designs. Beyond just machining work, Jack has been a close friend with many common interests. Jack is one of the few people within the Physics Department who similarly loves sports. I have enjoyed talking to Jack about many other things including cooking, woodworking, and dogs.

I also must thank Allan Schroeder, the supervisor for the main shop, and all of the talented machinists who work in the main shop. Work from the shop has contributed to nearly every aspect of all apparatus that I worked on as a graduate student. I am very grateful for Allan accommodating all of my projects, and even expediting progress on several occasions. For example, Allan arranged for the shop to machine all of the stainless steel components for our curved Halbach array over just several weeks. I have enjoyed working with all of the machinists individually on various projects, and I greatly appreciate their patience with me. I also greatly appreciate help from Ed Baez and Lanny Sandefur (and more recently Kenny Schneider) in the cryogenics shop. Ed and Lanny serviced vacuum pumps, checked various hardware for leaks, and provided liquid nitrogen and various gases. They often either loaned or gave us supplies when we needed something immediately.

The staff for the Center for Nonlinear Dynamics made my life as a graduate student vastly simpler. Olga Vera provided substantial assistance for many administrative manners including processing my appointments every semester. Olga, together with other staff including Marybeth Casias and Rachael Salge, greatly facilitated my progress in the lab by helping to process purchase orders and receiving packages.

I lastly would like to thank my family, notably my dog Simon. 


\title{
Magnetically Activated and Guided Isotope Separation
}

\author{
Thomas Rolf Mazur, Ph.D. \\ The University of Texas at Austin, 2014
}

Supervisor: Mark G. Raizen

This dissertation describes a proof-of-principle experiment demonstrating a technique for stable isotope enrichment called Magnetically Activated and Guided Isotope Separation (MAGIS) [1]. Over the past century a large number of enriched isotopes have become available, thanks largely to electromagnetic separators called calutrons that were developed during World War II. These isotopes have found applications across an array of fields including medicine, basic science, and energy. Due to substantial maintenance and operating costs, the United States decommissioned the last of its calutrons in 1998, leading to demand for alternative methods of isotope separation. Our experiment suggests the promise for MAGIS as a viable alternative for replenishing stockpiles previously provided by calutrons.

Our apparatus combines optical pumping with a scalable magnetic field gradient to enrich lithium-7 (Li-7) by suppressing lithium-6 (Li-6) throughput in a lithium atomic beam. We first evaporate lithium metal in a crucible in order to generate thermal, high flux beam. We then perform optical pumping on Li-6 atoms, magnetically polarizing a substantial fraction of Li-6 atoms into the entirely high-field seeking $2^{2} \mathrm{~S}_{1 / 2}, \mathrm{~F}=1 / 2$ ground state. The resultant beam then samples a magnetic field gradient produced by a $1.5 \mathrm{~m}$ long array of rare-earth permanent magnets bent over its length by $20 \mathrm{mrad}$. This geometry prevents high-field seeking lithium atoms from reaching the plane beyond the magnets, while efficiently deflecting low-field seeking atoms.

We measured Li-6 suppression - using independent techniques - along the plane after the magnets beyond a factor of 200 , corresponding to Li-7 enrichment to better than 
99.95\%. As apparatus-specific hindrances appeared to limit this suppression, we believe that we should achieve better enrichment on a commercial apparatus. We also measured both the absolute flux beyond the single, 1.5 in tall magnet array and the efficiency for guiding feedstock material to the collection plane. Given the planar configuration for the field gradient, the flux that we measured should scale linearly with both magnet height and the number of arrays surrounding the source. Our measurements therefore indicate that - at source temperatures that we actually investigated - a commercial apparatus fitting within a volume of just several cubic meters should yield hundreds of grams of enriched (to beyond 99.95\%) Li-7 per year. In addition, we observed a competitive ratio between collected material and feedstock with greater than $20 \%$ of lithium incident upon the magnet array reaching beyond the magnets.

Benchmarking our work against the calutron, we demonstrated comparable enrichment in a manner that should scale to the production of similar quantities. In contrast, however, MAGIS should require vastly less energy input. While calutrons required massive currents for maintaining a static magnetic field over a substantial area, the only non-shared energy expense for MAGIS is the cost for running the low power lasers for optical pumping. Via additional analysis, we have supplemented this proof-of-principle experiment with schemes for applying MAGIS to over half of the stable isotopes in the periodic table. Due to the success of this demonstration and the broad applicability of the principles, we believe that MAGIS will play an important role in the future of stable isotope enrichment. 


\section{Table of Contents}

Acknowledgments $\quad$ iv

Abstract $\quad$ viii

List of Tables $\quad$ xii

List of Figures $\quad$ xiii

$\begin{array}{lll}\text { Chapter 1. Introduction } & 1\end{array}$

1.1 Isotope Applications . . . . . . . . . . . . . . . . . . . 1

1.2 Calutrons ............................. 4

1.3 Alternative Techniques and MAGIS . . . . . . . . . . . . . 10

$\begin{array}{lll}\text { Chapter 2. Application to Lithium } & 14\end{array}$

2.1 Lithium Characteristics . . . . . . . . . . . . . . . 16

2.2 Magnetic Guide . . . . . . . . . . . . . . . . . . . . 24

2.2.1 Halbach Arrays and One-Sided Flux . . . . . . . . . . . . . . . . . 24

2.2 .2 Curved Halbach Array . . . . . . . . . . . . . . . . . . . . . . . . . 28

2.3 Numerical Simulations . . . . . . . . . . . . . . . . . . 33

$\begin{array}{lll}\text { Chapter 3. } & \text { Experiment Overview } & 41\end{array}$

3.1 Atomic Source . . . . . . . . . . . . . . . . . . . . . . 41

3.1.1 Various Iterations . . . . . . . . . . . . . . . . . . 41

3.1.2 Source Aperture . . . . . . . . . . . . . . . . . . 48

3.2 Laser System . . . . . . . . . . . . . . . . . . . . . 50

3.2.1 Commercial System . . . . . . . . . . . . . . 50

3.2 .2 Home-built System . . . . . . . . . . . . . . . . . . 55

3.3 Beam Line and Optical Pumping . . . . . . . . . . . . . . . . 58

3.3.1 Optical Setup . . . . . . . . . . . . . . . 61

3.3.2 Variable Beam Slit . . . . . . . . . . . . . . . . . . 64

3.4 Magnetic Guide . . . . . . . . . . . . . . . . . . . 66

3.4.1 Design and Construction . . . . . . . . . . . . 66

3.4 .2 Field Measurements . . . . . . . . . . . . . . . 73 
3.5 Detection . . . . . . . . . . . . . . . . 77

3.5.1 Relative Abundances: RGA and Fluorescence . . . . . . . . 78

3.5.2 Throughput: Wire Detector and Thickness Monitor . . . . . . 80

$\begin{array}{lrr}\text { Chapter } 4 . & \text { Measurements } & 89\end{array}$

4.1 Maximizing Efficiency . . . . . . . . . . . . . . . . . . . . . . . . . . . . . 89

4.2 Throughput and Efficiency . . . . . . . . . . . . . . . . 96

4.3 Enrichment . . . . . . . . . . . . . . . . . . . . . . . 110

4.4 Extraneous Measurements . . . . . . . . . . . . . . . . 130

Chapter 5. Apparatus Scaling, Beyond Lithium, and Conclusions 134

$\begin{array}{lr}\text { Bibliography } & 143\end{array}$ 


\section{List of Tables}

5.1 Optical pumping details for 27 elements, corresponding to 129 isotopes. . 142 


\section{List of Figures}

1.1 Simplified schematic of a calutron. . . . . . . . . . . . . . . . 6

1.2 Enrichment factors for isotopes enriched at ORNL. . . . . . . . . . 7

1.3 Collection rates for isotopes enriched at ORNL. . . . . . . . . . . . 8

1.4 Productivity comparison for isotopes enriched at ORNL. . . . . . . . . . 9

1.5 General overview of MAGIS. . . . . . . . . . . . . . . . . 13

2.1 Lithium vapor pressure in relevant temperature range. . . . . . . . . . . 16

2.2 Spectra summary for Li-6 and Li-7 ground and first excited states. . . . . 18

2.3 Breit-Rabi diagram for the Li-6 ground state. . . . . . . . . . . . . . . . 19

2.4 Summary of transition strengths for Li-6 D1 and D2 lines. . . . . . . . . 21

2.5 Overview of undulators used for generating synchrotron radiation. . . . . 25

2.6 Characteristics of Halbach arrays. . . . . . . . . . . . . . . . 26

2.7 Outline for guide geometry. . . . . . . . . . . . . . . . . . 30

2.8 Guide geometry considerations. . . . . . . . . . . . . . . . . 31

2.9 Optimizing guide characteristics. . . . . . . . . . . . . . . . 32

2.10 Overview of initial phase space for incidence angles and speeds. . . . . . . 34

2.11 Overview of terminal phase space. . . . . . . . . . . . . . . . 35

2.12 Trajectory fractions for given speeds before (red) and after (blue) magnet panel. . . . . . . . . . . . . . . . 36

2.13 Distribution for (weighted) trajectories reaching beyond magnets. . . . . 37

2.14 Distribution for (weighted) trajectories hitting magnets. . . . . . . . . . 38

2.15 Visual summary of terminal trajectories for different speed classes. . . . . 39

2.16 Comparing simulated throughput for $m_{J}=+1 / 2$ and $m_{J}=-1 / 2$ atoms for various source positions. . . . . . . . . . . . . . . . . 39

2.17 Summary of predicted throughput traces for different source positions. $\quad$. 40

3.1 First generation source $(\mathrm{G} 1) \ldots \ldots \ldots \ldots$. . . . . . . . . . . 42

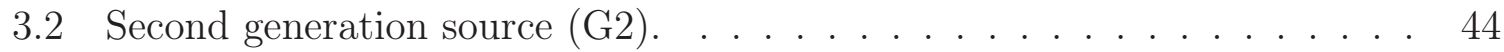

3.3 Third generation source $(\mathrm{G} 3) . \ldots \ldots \ldots$. . . . . . . . . . 47

3.4 Source aperture used for lower temperature measurements (primarily with G1). . . . . . . . . . . . . . . . . . . 49

3.5 Heated source aperture used for higher temperature measurements (using G2 and G3). . . . . . . . . . . . . . . . . . 51

3.6 Laser system used for bulk of measurements. . . . . . . . . . . . . 52 
3.7 Error signals produced for stabilizing the spectroscopy and seed lasers. . 54

3.8 Home-built laser system. . . . . . . . . . . . . . . . . . . 55

3.9 Tapered amplifier assembly. . . . . . . . . . . . . . . . . 57

3.10 Beam line for optical pumping. . . . . . . . . . . . . . . . . . 59

3.11 Laser beam path for optical pumping. . . . . . . . . . . . . . . . 62

3.12 Spectrum broadening using an EOM. . . . . . . . . . . . . . . . 64

3.13 First aperture system used in the optical pumping chamber. . . . . . . . 65

3.14 Variable slit used in the optical pumping chamber. . . . . . . . . . . . . . 66

3.15 Magnet panel assembly. . . . . . . . . . . . . . . . . 67

3.16 Aperture at magnet entry. . . . . . . . . . . . . . . . . . 69

3.17 Photos of the magnet array. . . . . . . . . . . . . . . . . 70

3.18 Original plans for stacking panels. . . . . . . . . . . . . . . 71

3.19 Assembly for epoxying magnets. . . . . . . . . . . . . . . . . 72

3.20 Alternative assembly for epoxying magnets. . . . . . . . . . . . . . . 73

3.21 Sample field measurements. . . . . . . . . . . . . . . . . . . . . . . 74

3.22 Main chamber. . . . . . . . . . . . . . . . . . 75

3.23 Detector layout for throughput analysis. . . . . . . . . . . . . . . . 77

3.24 Fluorescence setup. . . . . . . . . . . . . . . . . . . . . . . 79

3.25 Wire detector design. . . . . . . . . . . . . . . . . 81

3.26 Rhenium temperature as a function of current. . . . . . . . . . . . . 82

3.27 Wire detector for lithium detection in supersonic beams. . . . . . . . . . 84

3.28 Thickness monitor translation mechanism. . . . . . . . . . . . . . . . . 86

3.29 Complete apparatus. . . . . . . . . . . . . . . . . . . . 88

4.1 Calibration for rotation angle of atomic source. . . . . . . . . . . . . . 89

4.2 Maximizing apparatus efficiency via wire detector. . . . . . . . . . . . . 90

4.3 RGA signal dependence on source position. . . . . . . . . . . . . . . . . . 92

4.4 Wire detector traces as a function of source temperature. . . . . . . . . . 94

4.5 Determining relevant spatial extent of atomic beam in optical pumping chamber. . . . . . . . . . . . . . . . . 95

4.6 Overview of procedure for estimating throughput. . . . . . . . . . . 97

4.7 Response function measurement. . . . . . . . . . . . . . . . . . . . . . 98

4.8 Summary of throughput measurements using natural lithium. . . . . . . 100

4.9 Summary of throughput measurements using enriched Li-6. . . . . . . . . 102

4.10 Overview of atomic beam geometry. . . . . . . . . . . . . . . . . 103

4.11 Translating thickness monitor across optical pumping region. . . . . . . . 104

4.12 Lithium coating on magnet aperture (after removing magnets from chamber). . . . . . . . . . . . . . 106 
4.13 Various measurements of flux in optical pumping region as function of source temperature. . . . . . . . . . . . . . . . . . 107

4.14 Overview of procedure for estimating guide efficiency. . . . . . . . . . 109

4.15 Wire scan traces with (B) and without (A) optical pumping using enriched Li-6. . . . . . . . . . . . . . . . . . . . . . . 110

4.16 Quotients of traces with and without optical pumping (using enriched Li-6).111

4.17 Wire scan traces (and corresponding quotients) with and without optical pumping using natural lithium. . . . . . . . . . . . . . . 112

4.18 Comparison of wire scan traces when tuning the optical pumping laser to the $\mathrm{F}=3 / 2$ and $\mathrm{F}=1 / 2$ ground states. . . . . . . . . . . 113

4.19 Background-subtracted RGA spectra in presence and absence of optical pumping (using enriched Li-6). . . . . . . . . . . . . . . . . . . . 114

4.20 Dependence of Li-6 suppression on total laser power (using enriched Li-6). 115

4.21 Dependence of Li-6 suppression on spectral broadening and laser beam width. . . . . . . . . . . . . . . . . . . . 116

4.22 Overview of difficulty in using RGA for analysis of Li-6 suppression when using natural lithium. . . . . . . . . . . . . . . . . . 118

4.23 Enhancement of fluorescence signal in presence of repumping beam. . . . 119

4.24 Depletion dependence on source temperature (for natural lithium). . . . . 121

4.25 Outline of procedure for processing fluorescence images. . . . . . . . . . . 122

4.26 Overview of dependence of depletion value on cropping choice. . . . . . . 124

4.27 Depletion dependence on laser power and various powers applied to EOM (for natural lithium). . . . . . . . . . . . . . . . . . . 125

4.28 Summary of best and worst depletion values obtained from fluorescence measurements. . . . . . . . . . . . . . . . . . . . 127

4.29 Spatial dependence of depletion for various powers applied to EOM. . . . 128

4.30 Estimates for atomic density in pumping region. . . . . . . . . . . 130

4.31 Sample depletion when pumping on the D2 line. . . . . . . . . . . . . . 132

4.32 Sample fluorescence measured from above the optical pumping cross on a CCD . . . . . . . . . . . . . . . . . . . 133

5.1 Throughput summary and apparatus scaling. . . . . . . . . . . . . . 136

5.2 Comparison of vapor pressures among metallic elements. . . . . . . . . 137

5.3 More general magnetic field configuration for enriching arbitrary isotopes. 138

5.4 Summary of isotopes identified thus far as candidates for MAGIS. . . . . 141 


\section{Chapter 1}

\section{Introduction}

\subsection{Isotope Applications}

Infrastructure for enriching stable isotopes for large-scale use began in the 1940s. Since that time, stable isotopes have found a multitude of applications across a diverse array of fields including energy, medicine, basic research, and national security, among others. For instance, isotopes of certain elements - having particular characteristics like favorable cross-sections for neutron absorption - serve important roles in nuclear energy. Pressurized water reactors use enriched boron-10 - a neutron absorber - in boric acid for controlling fission rates. These reactors simultaneously employ lithium hydroxide for regulating the $\mathrm{pH}$ of the cooling water accordingly. The lithium hydroxide must be highly enriched using lithium-7 (Li-7) as lithium-6 (Li-6) has higher likelihood for producing tritium via neutron capture [2]. Tritium in turn can produce tritiated water which poses environmental risks. In contrast, fusion reactors relying on deuteriumtritium fusion will likely employ large quantities of enriched Li-6 as a tritium breeder in blankets surrounding the core [3].

Beyond reactors, certain isotopes have been used for generating radioisotopes - whose decays emit usable energy - that fuel batteries. For example, bombarding nickel-62 targets with neutrons in a reactor produces nickel-63 (Ni-63), a beta-emitting radioisotope with half-life close to 100 years. In 2011, Ni-63 was one of the top-selling isotopes - accounting for over $\$ 500,000$ in revenue - for the Department of Energy's (DOE) Isotope Program [4]. Likely because of Ni-63 demand, the DOE projects less than five years remaining for its existing stockpile of Ni-62. Another isotope, neodymium-146, has been one of the most in-demand isotopes for the DOE since 2000 [5] ${ }^{1}$. Similarly

\footnotetext{
${ }^{1}$ The website for the National Isotope Development Center includes limited documentation on news related to stable isotope separation in the United States. See http://www.isotopes.gov/news/hot.html.
} 
to Ni-62, neodymium-146 produces promethium-147 - another viable beta-emitter for batteries - via neutron capture in reactors [6].

Recent work has suggested that the efficiency for fluorescent lighting can potentially be improved by more than $10 \%$ just by tailoring the relevant abundances of mercury isotopes in lamps [7]. In fluorescent bulbs an electric discharge excites mercury atoms in a vapor to a state that emits a $254 \mathrm{~nm}$ photon upon decaying. This UV radiation generates fluorescence when incident upon a phosphor coating that lines the bulb. While radiation trapping within the vapor limits the likelihood for $254 \mathrm{~nm}$ photons to reach the bulb, adjusting the isotopic mixture (along with other lamp parameters) can improve the escape rate for the radiation by more than $20 \%$. While to date the cost for enriched mercury isotopes - notably mercury-196 - has been prohibitively high for use in lamps, the broadly applicable methods demonstrated in this work suggest a cost-effective means for realizing these mixtures.

Stable isotopes pervade medicine, having both diagnostic and therapeutic applications. Stable isotopes often serve as precursors for generating radioisotopes that have applications in nuclear medicine. Technetium-99m (Tc-99m) - a gamma-emitting (140 $\mathrm{keV}$ ) radioisotope whose half-life is only six hours - accounts for most of the world's use of radioisotopes in nuclear medicine including millions of procedures in the United States every year [8]. Due to its short half-life and easy-to-detect gamma ray, Tc-99m is used as a tracer. In most procedures, Tc-99m attaches to a suitable molecule that targets an organ of interest where single-photon emission computed tomography maps the distribution for gamma ray emission. In myocardial infusion imaging, for instance, Tc-99m decays allow for mapping of blood perfusion into the heart.

Today nuclear reactors generate molybdenum-99 (Mo-99) - a by-product of uranium fission with a 66 hour half-life - that in turn decays to Tc-99m. When two reactors simultaneously shut down in 2009 for maintenance, hospitals faced critical shortages of Tc-99m. With shut-downs looming in the near future, novel approaches have been considered for producing Tc-99m. Certain approaches use stable molybdenum isotopes - including Mo-98 and Mo-100 - as targets for neutrons, protons, or photons in order to produce Mo-99 (or even Tc-99m directly) [9]. These facilities, however, will depend 
heavily on sources of enriched molybdenum. Thallium-201 (Tl-201, with just a three hour half-life) is a similar radioisotope that has similarly been used as a tracer for heart imaging. Proton bombardment of Tl-203 in cyclotrons yields lead-201 which in turn decays to Tl-201. Likely because of uncertainty related to Tc-99m sources, Tl-203 has been labeled as one of the most important stable isotopes [4].

Stable isotopes similarly provide the supply for many important radioisotopes for positron emission tomography. Gallium-68 (Ga-68) has recently garnered significant attention for being a versatile radioisotope for PET imaging that has a short half-life (less than one hour) while exposing patients to lower doses of radiation [10,11]. Similarly to Mo-99, germanium-68 - a by-product of bombarding Ga-69 (stable) with protons serves as a longer half-life generator for Ga-68. Due to the increasing popularity of Ga-68 for PET imaging, demand for Ga-69 has increased substantially. DOE indicated in 2012 that its existing stockpiles will likely last for less than six years [4]. Copper-64 (Cu-64) is another important radioisotope for PET imaging. Its longer half-life (over twelve hours) allows for more manageable distribution. Proton bombardment of Ni-64 (stable) in cyclotrons can produce $\mathrm{Cu}-64$. Various properties of $\mathrm{Cu}-64$ - including the possibility for high-yield production via Ni-64 - have suggested its use in radioimmunotherapy [12].

Important work pursuing basic research has relied on substantial quantities of certain stable isotopes. For example, several collaborations have independently sought to measure a nuclear decay process called neutrinoless double beta decay. In this decay mode, two neutrons convert into pairs of protons and electrons without emitting neutrinos. Measuring this decay would indicate that the neutrino is Majorana, i.e. that it is its own anti-particle. Current experimental results have determined that the lower-bound for the half-life of this decay is beyond $10^{25}$ years. To suppress background rates while trying to observe this decay, collaborations therefore use massive quantities of observationally stable isotopes with extremely long half-lives. Stable isotopes that have been investigated include calcium-48, germanium-76, selenium-82, molybdenum100, and neodymium-150 [13]. For instance, the SNO + collaboration had suggested using hundreds of kilograms of neodymium-150 enriched to $80 \%$ until recently deciding to use tellurium-130 [14]. 
Another interesting application in physics has used highly-enriched silicon-28 in efforts toward defining the kilogram in terms of fundamental constants $[15,16]$. In particular, the Avogadro Project has counted the number of silicon atoms in a pair of one kilogram single-crystal silicon spheres. By measuring molar mass, lattice parameter, sphere volume and mass, and surface characteristics, the collaboration determined a value for the Avogadro constant which by definition gives the mass of a carbon-12 atom in kilograms. By constructing the spheres using silicon-28 enriched to $99.995 \%$, the experiment achieved lower uncertainty by accurately knowing the isotopic composition of the spheres.

The examples described above outline a miniscule fraction of the applications for stable isotopes. Most of these applications were likely not conceivable prior to World War II when infrastructure had not yet existed for producing many isotopes. Recent circumstances have led to increasing demands for many isotopes, thus motivating the development for novel methods of stable isotope production. Beyond just bolstering production for isotopes currently in demand, however, novel techniques should engender further applications. Our approach - Magnetically Guided and Activated Isotope Separation (MAGIS) - presents an efficient, scalable, and broadly applicable method that we think will supply isotopes for applications like those described above among many others.

\subsection{Calutrons}

Electromagnetic separation using machines called calutrons has been the most prolific method to date for enriching isotopes in terms of applicability. In 1930, Ernest Lawrence invented the cyclotron, which later developed into this general method for isotope separation based on ionization of atoms with electrons, and separation by the charge-to-mass ratio [17]. The calutrons, invented for the Manhattan Project in World War II, were later realized as general-purpose apparatus that could provide small quantities of most stable isotopes in the periodic table [18]. As shown in Fig. 1.1, a heated source first vaporizes feed material that begins in either elemental or compound form. An arc discharge ionizes a fraction of particles in this vapor, and then a large potential 
difference $V$ - typically close to $40 \mathrm{keV}$ - accelerates the ions into a large surrounding chamber. As evident from the Lorentz force, the resulting speed $v$ for an ion of mass $m$ is given by

$$
v=\sqrt{\left(\frac{2 e V}{m}\right)}
$$

where $e$ denotes the electron charge (indicating that typical speeds far exceed $1000 \mathrm{~m} / \mathrm{s}$ ). Large current-carrying coils surrounding the chamber generate a static magnetic field $B$ that bends the ions into circular trajectories via a centrifugal force. Again in accordance with the Lorentz force, the radius $r$ for a given ion is given by

$$
r=\frac{1}{B}\left(\frac{\sqrt{2 m V}}{e}\right)
$$

Early generation machines bent trajectories by $180^{\circ}$ over radii $r \sim 0.5 \mathrm{~m}$, requiring field strengths close to $1 \mathrm{~T}$. Ions then terminated on collection pockets that were typically spaced by several centimeters in accordance with (1.2). A key feature of the calutron is that all isotopes of a given element can be simultaneously enriched.

The calutron program in the United States developed at Oak Ridge National Laboratory (ORNL). Building the calutrons required substantial effort for overcoming a series of engineering obstacles [19]. For example, the design for the collection pockets - including shape and material - often needed to be tailored for a specific element in order to mitigate effects like erosion due to sputtering by the highly energetic ion beams. Also, focusing the large-current ion beams into the collection pockets proved challenging due to electrostatic repulsion. By compromising experimental parameters accordingly, however, calutrons achieved impressive enrichment while maintaining steady throughput. Generating the $1 \mathrm{~T}$ static field arguably posed the most severe obstacle. The cross-section for early calutrons measured nearly twelve feet by eight feet. To generate the field over these large dimensions, nearly ten tons of windings were used for the surrounding coils. In fact, in building the first machines for enriching uranium, ORNL borrowed thousands of tons of silver from the U.S. Treasury!

By 1987 the isotope separation program at ORNL had separated 235 isotopes of 56 elements. Extensive tables provide both collection rates and degrees of enrichment 


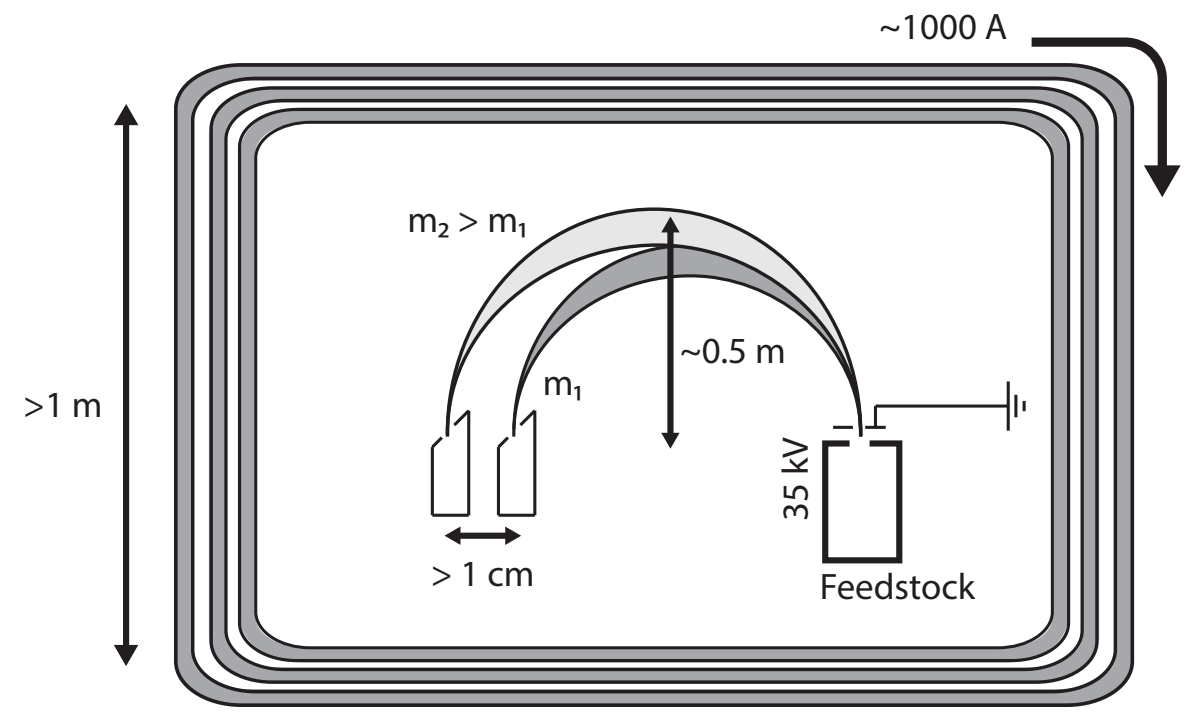

Figure 1.1: Simplified schematic of a calutron. A source first vaporizes and ionizes elemental material. A large voltage then extracts ions from the source into an enclosing chamber. A large static magnetic field produced by surrounding coils bends these ions into circular trajectories. The radius for a particular trajectory depends on the mass of the ion. All isotopes of an element can be simultaneously enriched by positioning collection pockets at suitable distances away from the source.

for isotopes processed by these calutrons. For reference, a figure-of-merit is that a single calutron typically operates its source at $25 \mathrm{~mA}$, ultimately processing $0.1 \mathrm{~mol}$ per operating day [20]. Multiplying this number by the relative abundance of an isotope gives a throughput estimate for that isotope per day. Enrichment factors $\digamma$ for many isotopes range between 100 and 1000 where

$$
\digamma \equiv \frac{N_{1} /\left(1-N_{1}\right)}{N_{0} /\left(1-N_{0}\right)}
$$

with $N_{0}$ and $N_{1}$ denoting the relative abundances of the isotope before and after enrichment.

Fig. 1.2 summarizes enrichment factors - extracted directly from an ORNL report - for isotopes that were produced between 1945 and 1984 at ORNL [21]. Data points give the average among enrichment factors - weighted by relative abundances - for isotopes of a particular element. Vertical error bars indicate the spread in enrichment factors for the isotopes of a given element, and horizontal error bars show the mass range spanned 


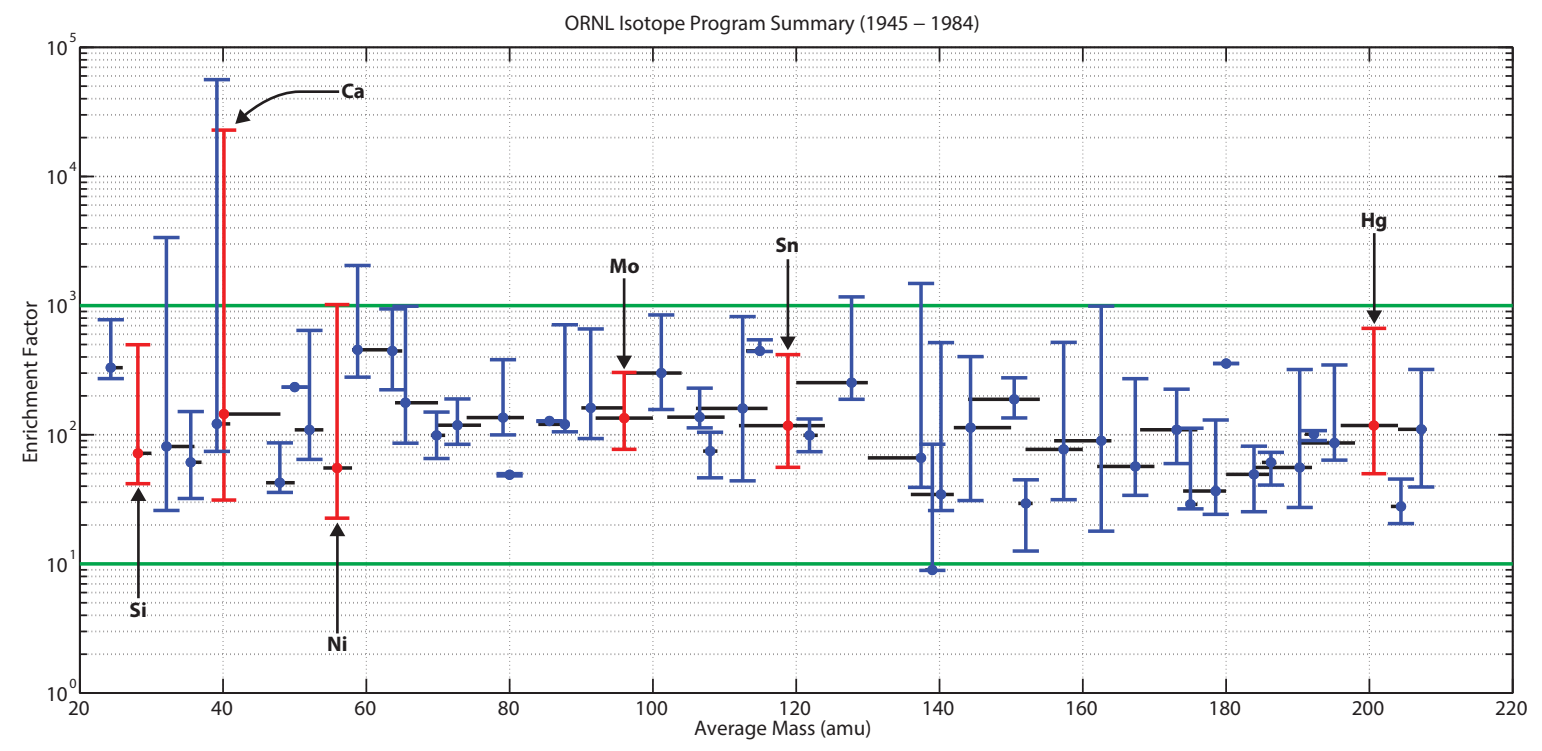

Figure 1.2: Enrichment factors for isotopes enriched at ORNL. Data points show the average enrichment factor for the isotopes of a particular element (weighted by relative isotopic abundances). For isotopes where tables specify a range for resulting enrichment, we choose the maximum degree of enrichment for calculating an enrichment factor. Vertical error bars show the range of enrichment factors that were achieved among the isotopes for an element. Horizontal lines show the mass ranges for the isotopes of the elements. Elements shown in red accounted for almost $40 \%$ of all production between 1945 and 1984. 


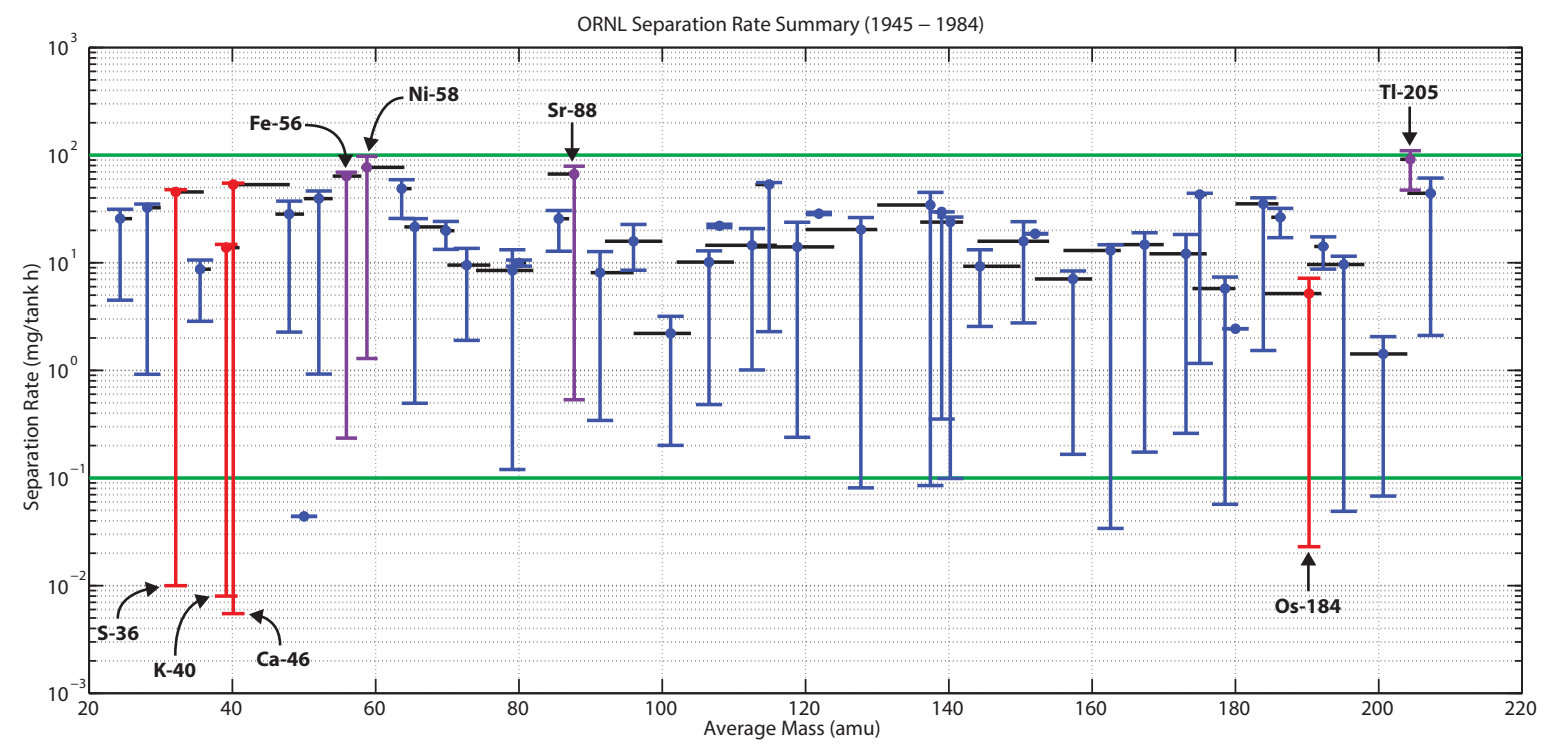

Figure 1.3: Collection rates for isotopes enriched at ORNL. Data points show weighted averages of collection rates $(\mathrm{mg} / \operatorname{tank} \mathrm{h})$ for isotopes of particular elements. See Fig. 1.2 for meaning of error bars. Elements shown in purple and red indicate the largest and smallest collection rates for isotopes that were enriched during this period.

by isotopes of that element ${ }^{2}$. During this period, isotopes for calcium, iron, mercury, tin, and silicon (shown in red) accounted for nearly $40 \%$ of all production at ORNL, with calcium alone accounting for more than $10 \%$.

The calutron program at ORNL enriched a few outliers - notably calcium-46, calcium-48, and potassium-40 - to factors well beyond 1000, although available records do not provide complete information concerning protocols for all isotopes. Alternative designs for calutrons enabled higher purities at the expense of ion throughput. Moreover, an enriched isotope collected during one stint could be further purified through a subsequent pass. Fig. 1.3 shows collection rates $\mathcal{R}$ for those isotopes summarized in Fig. 1.2 (again giving weighted averages), providing limited insight concerning the degreeof-difficulty for enriching certain isotopes. Not surprisingly, lower abundance isotopes - like sulfur-36, potassium-40, calcium-46, and osmium-184 (red) - exhibit correspondingly lower flux. While the throughputs for calcium isotopes (like those for isotopes of

\footnotetext{
${ }^{2}$ Vanadium and tantalum each have just two stable isotopes with one of the isotopes being less than $1 \%$ abundant.
} 


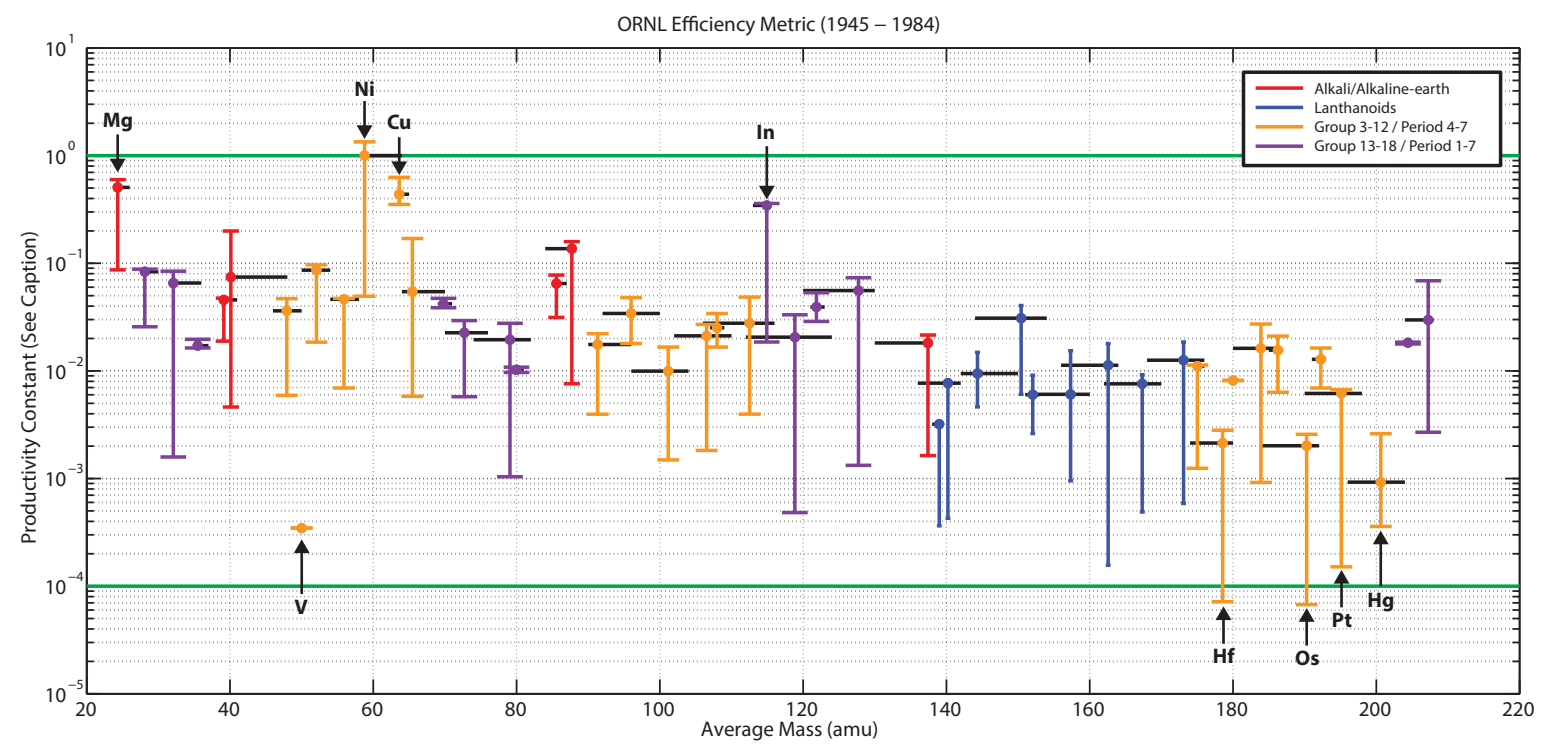

Figure 1.4: Productivity comparison for isotopes enriched at ORNL. Data points show products of weighted averages for the enrichment factors and molar collection rates among isotopes for particular elements. See Fig. 1.2 for meaning of error bars.

other elements) reflect the relative isotopic abundances, the exorbitant enrichment factors for calcium-46 and calcium-48 in comparison to other calcium isotopes (indicated in Fig. 1.2) imply isotope-specific protocols such as a subsequent stage using enriched material as feedstock. Data points shown in purple in Fig. 1.3 indicate the four highest throughput isotopes among those produced by the calutrons.

In an effort to assess the overall performance of the calutron in its application to various isotopes, we define productivity constants for the isotopes corresponding to the products of their enrichment factors $\digamma$ and molar separation rates $\mathcal{R}$. As shown in Fig. 1.4 a few elements - magnesium, nickel, copper, and indium - yield markedly higher constants. Among the lowest productivity isotopes include isotopes of refractory metals (notably vanadium, hafnium, osmium, and platinum) which vaporize at extremely high temperatures in elemental form. Fig. 1.4 partitions elements according to location on the periodic table, although no trends seem immediately evident.

While calutrons at ORNL were remarkably prolific, the United States decommissioned the last of its calutrons in 1998 due to high maintenance and operating costs [22]. The energy input for one machine - largely contributing to maintaining the $1 \mathrm{~T}$ 
static magnetic field - was large. For certain low abundance isotopes, the energy input exceeded 1 TJ for enriching just a gram of material. For example, enriching one gram of vanadium-50 from its natural abundance (0.25\%) to $36 \%$ would require over 4 TJ [23]. In this case, just the potential difference for extracting vanadium ions out of the source would require 50 GJ. Almost all of the remaining energy, however, would be consumed by the coils for maintaining the static field.

With calutrons no longer operating in the U.S. due to their inefficiency, concerns have grown as domestic stockpiles of many important isotopes have dwindled. In 2008, the Nuclear Science Advisory Committee (NSAC) - a committee that advises DOE on matters of nuclear science - held a workshop in order to investigate options for maintaining inventory of these isotopes among others [24]. A year later, NSAC released its final report: Isotope's For the Nation's Future: A long range plan [5]. The workshop and subsequent report projected remaining inventory in the U.S. for many isotopes, particularly identifying those with supplies that should last less than twenty years. A key point of emphasis of the report was the need for developing robust infrastructure for producing isotopes. A recommendation in its summary directly reads:

"Support a sustained research program in the base budget to enhance the capabilities of the isotope program in the production and supply of isotopes generated from reactors, accelerators, and separators."

\subsection{Alternative Techniques and MAGIS}

As implied by the NSAC report in 2008, no single method has emerged as a viable general alternative to the calutron in terms of degree of enrichment, scalability, and efficiency despite years of effort. Three criteria for any effective isotope separation technique include the ability to: (i) achieve purity that meets or exceeds market demand, (ii) scale an apparatus to the production of commercially relevant quantities, (iii) and operate efficiently, which entails maximizing the ratio between enriched material and feedstock, requiring sustainable power consumption, and being applicable to multiple elements. Defining absolute standards for these criteria is challenging both due to vari- 
ations in physical properties and also differences in applications and the concomitant value of various isotopes. The calutron, however, provides benchmarks for comparing performance. As mentioned previously, a figure-of-merit is that a single calutron can process 0.1 mol multiplied by the relative abundance of an isotope per day of operation while achieving enrichment factors between 100 and 1000 .

It is beyond the scope of this work to exhaustively address alternative separation techniques in detail. Gas centrifuges garner significant attention due to their application toward uranium enrichment. While centrifuges lend themselves to scaling, their application requires that either an element or a compound containing that element have sufficient vapor pressure near room temperature. For example, the only suitable uranium compound for use in centrifuges is uranium hexafluoride which is a highly toxic compound. Gaseous diffusion likewise requires that source material have high vapor pressure near room temperature. In contrast to centrifuges, however, diffusion requires many stages in order to achieve high separation, making this technique prohibitively expensive. While chemical methods sometimes enable substantial enrichment of large quantities, these techniques mostly consist of element-specific protocols. For instance, chemical exchange methods require two compounds for an element existing in immiscible fluid phases. In certain cases, atoms of an isotope might slightly favor one phase over another.

Other plasma-based techniques have presented more promising approaches to isotope separation. AVLIS (Atomic Vapor Laser Isotope Separation) selectively photoionizes atoms of a particular isotope, then extracts these ions using a modest voltage. AVLIS should be applicable to almost all elements, and has achieved significant enrichment [25]. Multiple lasers photoionize atoms of a given isotope: several lasers resonantly excite atoms into an excited state, and then a very high power laser ionizes those atoms. Another separation technique using isotope-selective ion-cyclotron resonances has demonstrated substantial separation while requiring much lower energy consumption in comparison to the calutron [26]. While these techniques present advantages over calutrons, neither has materialized in a large-scale operation for supplanting the calutron. 
Our approach - called Magnetically Activated and Guided Isotope Separation (MAGIS) - combines well-established principles within atomic physics [27]. Rather than generate a plasma like many other sources, we generate a large, effusive atomic flux into a large solid angle. In proximity to the source, we perform optical pumping, first described by Kastler in 1950, in order to magnetically polarize atoms of a target isotope [28]. We subsequently use a unique magnetic field gradient for efficiently guiding atoms of a desired isotope to a collection plane. Other magneto-optic systems combined similar principles toward isotope separation $[29,30]$. These systems, however, provided little enrichment with no apparent means of scaling production toward meaningful quantities or continuous use. Magneto-optic traps, for instance, offer no opportunities for macroscopic production, separating just fg/s generally [31]. Other work derived from a thermal beam demonstrated a change in the isotopic ratio of lithium by a substantially smaller amount than that reported in this work and achieved significantly lower throughput in a commercially unfeasible magnetic configuration.

Fig. 1.5 shows a schematic for MAGIS. By using a planar configuration for generating our field gradient, the solid angle subtended by the collection plane to the source can be engineered to be several steradians just by extending the height of this field array and adding additional arrays about the atomic source. The exact geometry for the field configuration will be tailored for enriching a particular isotope. In the geometry shown in Fig. 1.5, the field gradient bends slightly in order to obstruct lineof-sight between the source and collection region. Using optical pumping to polarize atoms of an undesired isotope into a high-field seeking state, the geometry will prevent those atoms from reaching the collection plane thus enriching a second isotope. A key aspect of optical pumping is that each atom of a targeted isotope needs to scatter only a few photons on average to changes its internal state. As a result, laser powers feasibly produced by inexpensive semiconductor lasers should enable many moles of material to be enriched per year.

This work summarizes a proof-of-principle experiment demonstrating the operating principles for MAGIS in application to Li-7 enrichment. By substantially suppressing Li-6 throughput in an atomic beam, we imply Li-7 purity on par with enrichment fac- 

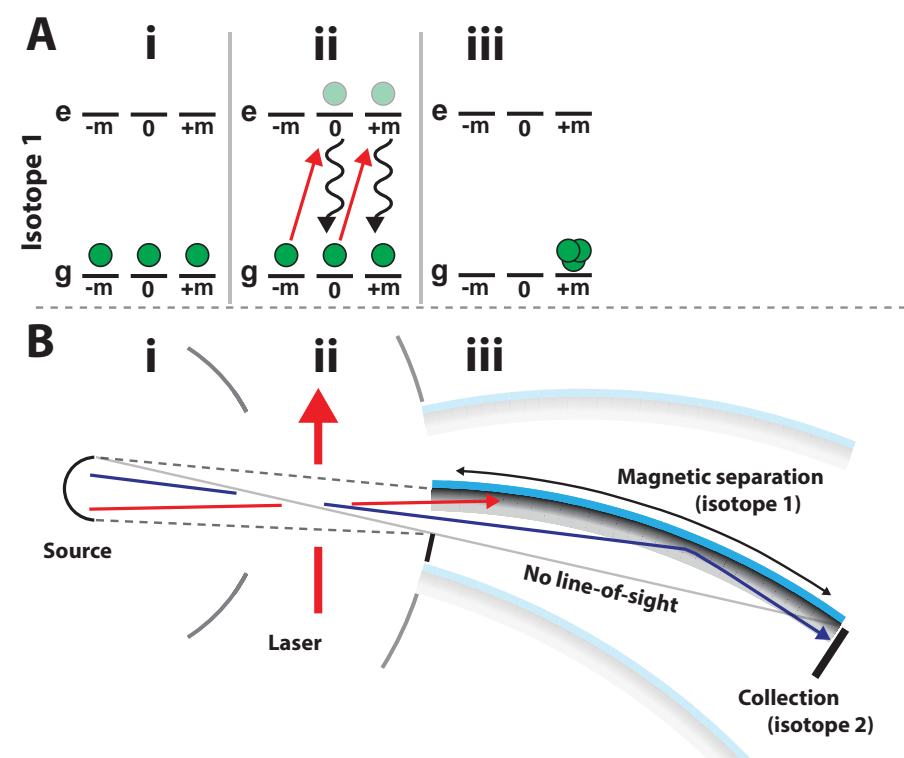

Figure 1.5: General overview of MAGIS. MAGIS is a three-step process including: (i) vaporization of elemental material in a source for producing an atomic flux into a large solid angle, (ii) magnetic polarization of atoms of one isotope via optical pumping, and (iii) enrichment by using a planar magnetic field gradient for filtering optically pumped atoms (either allowing or impeding passage to beyond the magnets).

tors generated by calutrons. In addition, we measure throughput that should feasibly scale to macroscopic quantities in a manner that efficiently collects feedstock. On a commercial scale apparatus, MAGIS should require modest energy input with the bulk of its expenses being shared among the negligible contributions for calutron operation (i.e. vacuum pumps and feedstock vaporization). Like the calutron, MAGIS should be broadly applicable: most atoms are paramagnetic in either their ground state or in a long-lived metastable state, and inexpensive lasers span wavelengths of interest for optical pumping of atoms. 


\section{Chapter 2}

\section{Application to Lithium}

As mentioned previously, both lithium isotopes - Li-6 and Li-7 with $7.5 \%$ and 92.5\% abundances respectively - have important applications. Enriched Li-6 functions as a tritium breeder in blankets that surround the core of certain power reactors based on deuterium-tritium (D-T) fusion. Li-6 has a higher cross-section for neutron capture (compared to Li-7) across the energy range for neutrons produced by this reaction [2]. Certain lithium compounds that had been considered for tritium breeding would require Li-6 enriched to up to 90\% [3]. Reactors like ITER (International Thermonuclear Experimental Reactor) will require $300 \mathrm{~g}$ of tritium per day for producing $800 \mathrm{MW}$ of electrical power ${ }^{1}$. Breeders thus should need massive amounts of enriched Li-6.

On the other hand, highly enriched Li-7 has been used in the cooling water for pressurized water reactors. Boric acid in the cooling water contains boron-10 which regulates the nuclear reaction in the core. Lithium hydroxide - a highly basic compound - prevents corrosion by modulating the acidity of the coolant (containing boric acid). The lithium hydroxide requires Li-7 enriched to beyond $99.9 \%$ in order to limit tritium production that in turn can produce tritiated water which poses environmental hazards [32]. Particular designs for novel molten salt reactors - that use fluid fuels consisting of fissile materials and carrier salts - will require massive quantities of Li-7 (many tons) enriched to beyond $99.995 \%$ [33].

In 2013, the U.S. Government Accountability Office (GAO) released a report recommending that the Secretary of Energy take action in order to ensure stable supplies of Li-7 in the future [34]. The U.S. requires nearly $300 \mathrm{~kg}$ per year of enriched Li-7 for its 65 pressurized water reactors (accounting for $13 \%$ of the country's electricity).

\footnotetext{
${ }^{1}$ For instance, see https://www.iter.org/mach/tritiumbreeding.
} 
The U.S. currently does not produce Li-7 domestically, and instead relies on importing material from Russia and China. These foreign supplies present risks as the U.S. has little knowledge of exact production capabilities. Between 1955 and 1963, the U.S. did produce lithium isotopes at ORNL. At this time, the U.S. had focused on stockpiling Li-6 for use in thermonuclear weapons. Today the U.S. has miniscule Li-7 stockpiles (totaling less than $2000 \mathrm{~kg}$ ) in chemical forms that require further processing for use in reactors.

To date, lithium isotopes have primarily been enriched - both domestically during the stint at ORNL and abroad in Russia and China - by a chemical method called COLEX (column exchange method). Chemical exchange processes like COLEX initially distribute an element as compounds among two immiscible phases. Flowing one phase across the other, atoms of one isotope preferentially migrate to one phase while atoms of another migrate to the other phase. COLEX uses a lithium-mercury amalgam and lithium hydroxide as the phases, with Li-6 being slightly enriched in the amalgam phase (and likewise Li-7 being distilled in water) after a single stage [2]. With the enrichment per stage being miniscule, ORNL required massive volumes of mercury for achieving purities of interest. ORNL had apparently considered hundreds of alternative systems in order to circumvent using mercury, but none yielded comparable performance. The U.S. shutdown its COLEX program in 1963 largely due to concerns relating to the hazards of handling these quantities of mercury. Subsequent reports indicated that ORNL had used close to 25 million pounds of mercury at its facility. Over 2 million pounds had been either unaccounted for or lost to the environment [35]. Even under dire circumstances, the likelihood for again utilizing COLEX seems very low due to immense environmental concerns.

Alternative methods that have been considered for lithium enrichment include displacement chromatography, thermal diffusion, and electromagnetic separation. None have yet demonstrated the ability to yield comparable performance to chemical exchange in terms of both throughput and purity. Laser-based methods have also been applied toward lithium enrichment. The earliest efforts used two lasers for selectively exciting then ionizing lithium molecules of a particular isotope [36]. While indicating substantial 


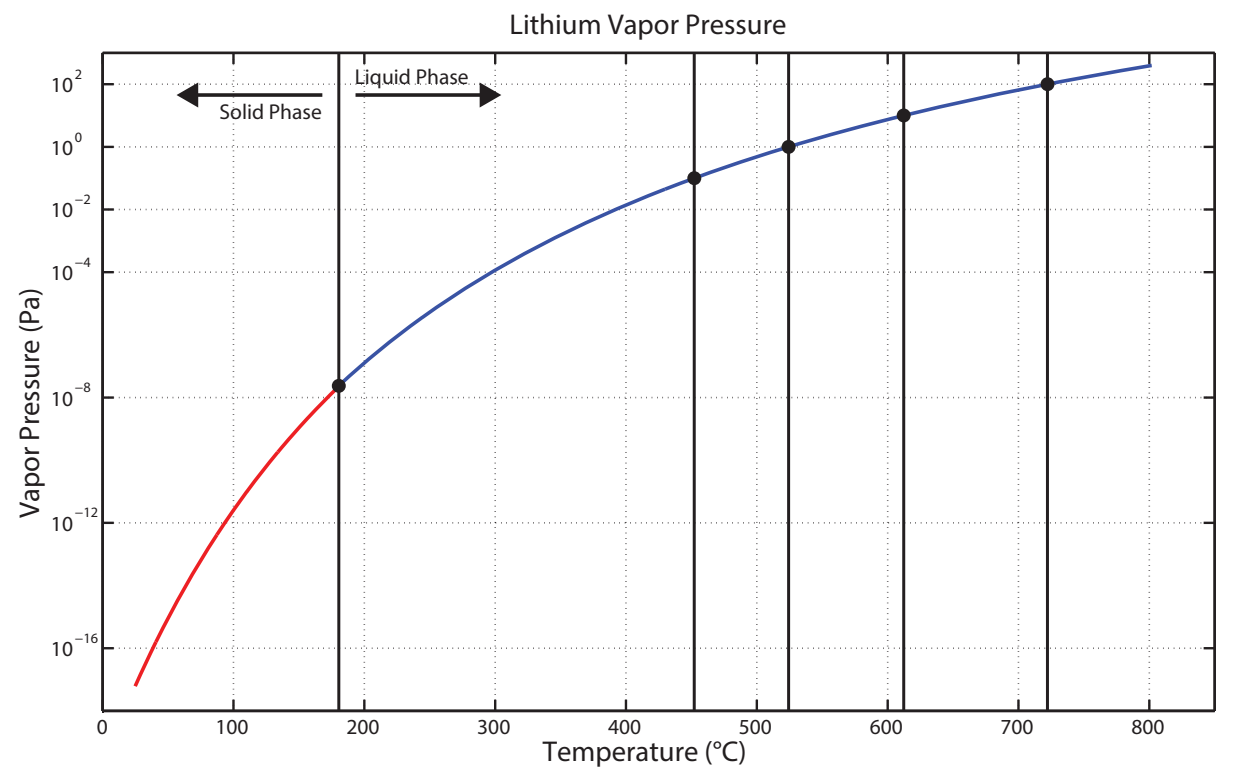

Figure 2.1: Lithium vapor pressure in relevant temperature range. Red and blue distinguish solid and liquid phases, respectively. Black lines show vapor pressures of interest including: phase transition, 0.1 Pa, $1 \mathrm{~Pa}, 10 \mathrm{~Pa}$, and $100 \mathrm{~Pa}$.

separation, this work yielded low quantities. Subsequent work similarly combined twolaser photoionization using atomic lithium, but again showed little promise for scaling to meaningful quantities [37-39]. In this work, we yield better enrichment than prior laser-based methods while measuring throughput that could potentially scale toward kilogram per year production in a single machine.

\subsection{Lithium Characteristics}

Under standard temperature and pressure, lithium is the lightest metal with the lowest density among solid elements $\left(\rho=0.534 \mathrm{~g} / \mathrm{cm}^{3}\right)$. Between room temperature and its melting point $\left(180^{\circ} \mathrm{C}\right)$, the vapor pressure $P$ for lithium as a function of temperature $T$ is reasonably given by

$$
P=10.673-8310 / T
$$


with $P$ and $T$ given in $\mathrm{Pa}$ and $\mathrm{K}$ respectively [40]. Likewise, from its melting point to beyond $1000 \mathrm{~K}$, another fit to data reliably describes the vapor pressure as

$$
P=10.061-8023 / T
$$

Fig. 2.1 shows the vapor pressure for lithium across the temperature range of interest for this work. The vapor pressure spans close to twenty orders of magnitude between the peak operating temperature that we investigate with our source $\left(750{ }^{\circ} \mathrm{C}\right)$ and typical temperatures for the rest of the apparatus $\left(\sim 20{ }^{\circ} \mathrm{C}\right)$. We thus can readily produce large atomic flux at the source with minimal concern for high-field seeking lithium atoms to reach beyond the magnetic field gradient as lithium atoms should reliably condense on any subsequent surfaces they encounter.

Lithium (with electronic spin $S=1 / 2$ ) has only two naturally occurring stable isotopes: Li-6 and Li-7 with $7.6 \%$ and $92.4 \%$ abundances, respectively. Li-6 is a fermion with nuclear spin $I=1$, while Li-7 - having four neutrons - is a boson with nuclear spin $I=3 / 2$. Like other alkali atoms, lithium has a a pair of strong $\mathrm{D}$ lines between the ground $2^{2} \mathrm{~S}$ state and the excited $2^{2} \mathrm{P}$ state. Accounting for the fine structure of the excited state, the D1 line particularly corresponds to the transition between the ${ }^{2} \mathrm{~S}_{1 / 2}$ and ${ }^{2} \mathrm{P}_{1 / 2}$ states, while the $\mathrm{D} 2$ line is the transition between the ${ }^{2} \mathrm{~S}_{1 / 2}$ and ${ }^{2} \mathrm{P}_{3 / 2}$ states. Fig. 2.2 - based entirely on work by Noble, et al. - outlines the spectra for the D lines in Li-6 and Li-7 [41].

The fine structure splitting between the ${ }^{2} \mathrm{P}_{1 / 2}$ and ${ }^{2} \mathrm{P}_{3 / 2}$ states is close to 10.05 $\mathrm{GHz}$ and in agreement for both isotopes. With the isotope shift for the D1 line between the isotopes being nearly $10.53 \mathrm{GHz}$, the frequency difference between the D2 line for Li-6 and the D1 line for Li-7 is only $\sim 480 \mathrm{MHz}$. Both isotopes have hyperfine structure (due to their nuclear spin), with the Li-6 ground state notably splitting into a pair of states close to $228 \mathrm{MHz}$ apart with total spins given by $F=3 / 2$ and $F=1 / 2^{2}$. Due mainly to the larger extent of the electronic wavefunctions, the magnetic dipole and electric quadrupole constants for the ${ }^{2} \mathrm{P}_{1 / 2}$ and ${ }^{2} \mathrm{P}_{3 / 2}$ states are significantly smaller

\footnotetext{
${ }^{2}$ Alternatively, the magnetic dipole constant for the ${ }^{2} \mathrm{~S}_{1 / 2}$ state in Li-6 is about $152 \mathrm{MHz}$.
} 


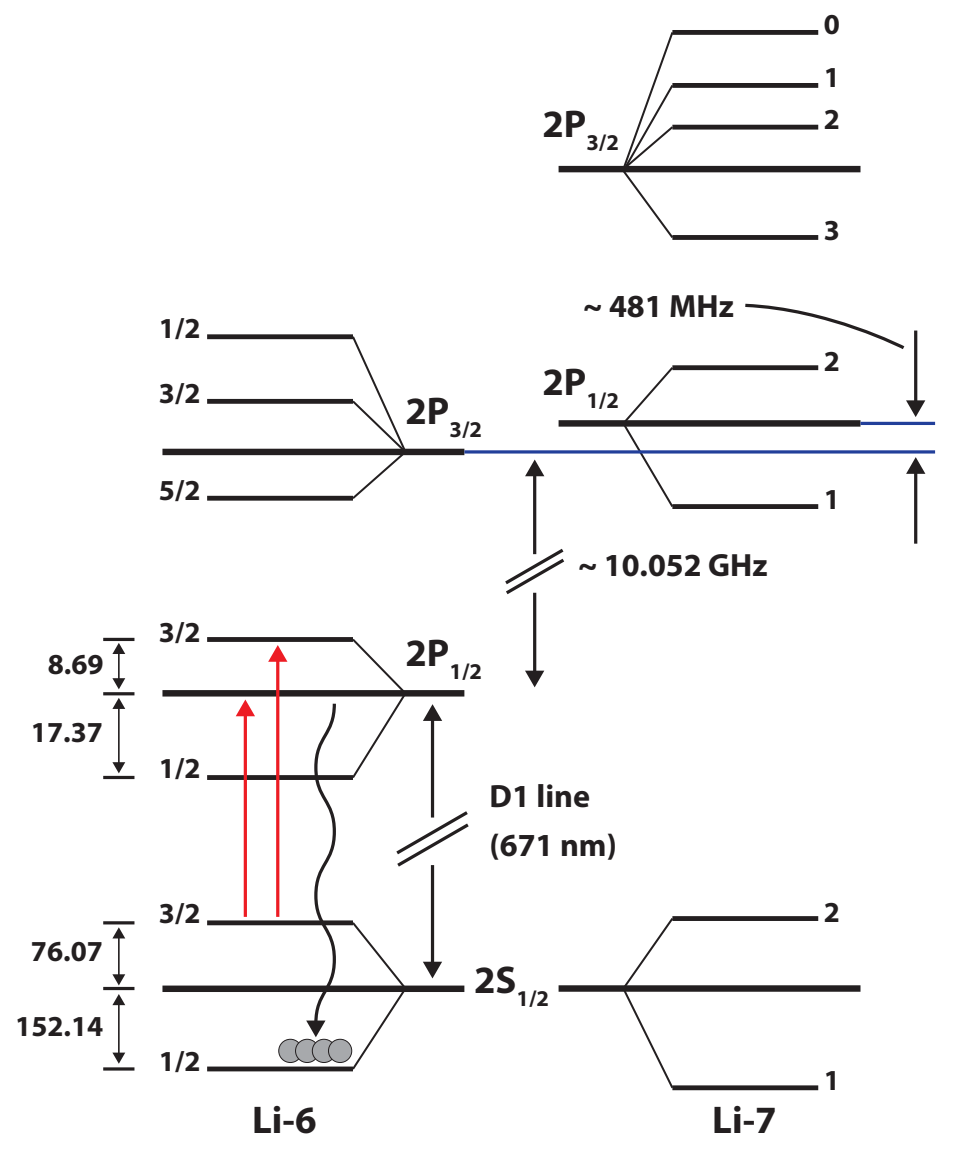

Figure 2.2: Spectra summary for Li-6 and Li-7 ground and first excited states. We perform optical pumping on the Li-6 D1 line, particularly driving atoms out of the $F=3 / 2$ state into the $F=1 / 2$ state. The hyperfine splitting of the Li- 6 ground state is close to $228 \mathrm{MHz}$. The isotope shift for the D1 line is nearly $10.5 \mathrm{GHz}$. By chance, the difference between the Li-7 D1 line and Li-6 D2 line is less than $500 \mathrm{MHz}$.

than for the ${ }^{2} \mathrm{~S}_{1 / 2}$ state. For the ${ }^{2} \mathrm{P}_{1 / 2}$ state (whose electric quadrupole constant vanishes) the magnetic dipole constant is only $17.4 \mathrm{MHz}$, while the magnetic dipole and electric quadrupole constants for the ${ }^{2} \mathrm{P}_{3 / 2}$ state are just -1.2 and $-0.1 \mathrm{MHz}$, respectively [42].

Fig. 2.3 shows the Zeeman splitting of the Li-6 ground state in the presence of an external magnetic field. An external magnetic field interacts with the total electronic and nuclear magnetic moments of the ${ }^{2} \mathrm{~S}_{1 / 2}$ state of a lithium atom. The Hamiltonian that describes the interaction energy between the field $\vec{B}$ and these magnetic moments is given by

$$
H_{Z}=-\left(\vec{\mu}_{J}+\vec{\mu}_{I}\right) \cdot \vec{B}
$$




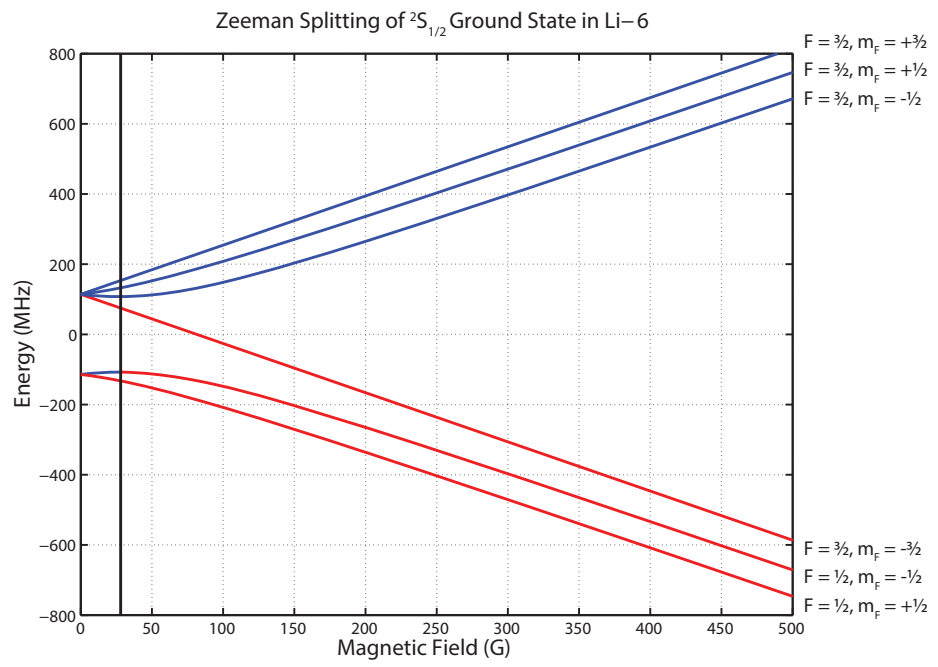

Figure 2.3: Breit-Rabi diagram for Li-6 ground state. Red and blue lines denote highand low-field seeking regions respectively. Beyond $40 \mathrm{G}$, the $F=1 / 2$ state (corresponding to the lowest two curves) is entirely high-field seeking.

where $\vec{\mu}_{J}$ and $\vec{\mu}_{I}$ denote the total electronic (orbital plus spin) and nuclear magnetic moments, respectively. The total magnetic moment $\vec{\mu}$ can be expressed in terms of the electronic $\vec{J}$ and nuclear $\vec{I}$ spins by

$$
\vec{\mu}=\vec{\mu}_{J}+\vec{\mu}_{I}=\frac{\mu_{B}}{\hbar}\left(g_{J} \vec{J}+g_{I} \vec{I}\right)
$$

where $\mu_{B}$ is the Bohr magneton, $\hbar$ is the reduced Planck constant, and $g_{J}$ and $g_{I}$ denote electronic and nuclear Landé-g factors.

To most generally determine the Zeeman splitting, the Hamiltonian describing both hyperfine and Zeeman interactions should be considered. The hyperfine interactions for the energy levels corresponding to the D lines is reasonably described by the Hamiltonian

$$
H_{h f s}=A_{h f s} \vec{I} \cdot \vec{J}+B_{h f s} \frac{3(\vec{I} \cdot \vec{J})^{2}+3(\vec{I} \cdot \vec{J}) / 2-I(I+1) J(J+1)}{2 I(2 I-1) J(2 J-1)}
$$

where $A_{h f s}$ and $B_{h f s}$ denote the magnetic dipole and electric quadrupole constants for a given state $[42,43]$. As the electric quadrupole interaction contributes only toward states with $I, J \geq 1$, the Hamiltonian describing the Zeeman and hyperfine interactions for the Li-6 ground state can be written as

$$
H=H_{h f s}+H_{Z}=A_{h f s} \vec{I} \cdot \vec{J}-\frac{\mu_{B}}{\hbar}\left(g_{J} \vec{J}+g_{I} \vec{I}\right) \cdot \vec{B}
$$


For cases where either $J=1 / 2$ or $I=1 / 2$, diagonalizing the above Hamiltonian yields an analytical expression. In particular, when $J=1 / 2$ as in the case of the ${ }^{2} \mathrm{~S}_{1 / 2}$ state (2.6) yields energies for the resulting states $\left|F_{ \pm}=I \pm 1 / 2, m_{F}\right\rangle$ given by

$$
\frac{E\left(F_{ \pm}, m_{F}\right)}{\Delta E_{h f s}}=-\frac{1}{2(2 I+1)}-\left(\frac{m_{F}}{g_{J} / g_{I}-1}\right) x \pm \frac{1}{2} \sqrt{1+\left(\frac{4 m_{F}}{2 I+1}\right) x+x^{2}}
$$

where $\Delta E_{h f s}$ is the energy difference between the hyperfine states in zero field and $x$ denotes a dimensionless parameter defined by

$$
x \equiv \frac{\left(g_{J}-g_{I}\right) \mu_{B} B}{\Delta E_{h f s}}
$$

The result given by (2.7) - which is valid for any $I$ when $J=1 / 2$ - is the Breit-Rabi formula $[44,45]$. The hyperfine splitting $\Delta E_{h f s}$ can be determined by diagonalizing $(2.5)$ and finding the difference between the resulting eigenvalues $[42,43]$. For the ${ }^{2} \mathrm{~S}_{1 / 2}$ state, diagonalizing (2.5) gives

$$
E_{h f s}(F)=\frac{1}{2} A_{h f s} K
$$

where

$$
K=F(F+1)-I(I+1)-J(J+1)
$$

As shown in Fig. 2.3 three states within the $F=3 / 2$ hyperfine state of the Li-6 ${ }^{2} \mathrm{~S}_{1 / 2}$ state (corresponding to $m_{F}=-1 / 2,+1 / 2,+3 / 2$ ) monotonically increase in energy in increasing magnetic fields. In order to suppress Li-6 throughput beyond our magnetic field gradient, we thus must optically pump Li-6 atoms out of these low-field seeking states. In contrast, the state $\left|F=3 / 2, m_{F}=-3 / 2\right\rangle$ along with both states in the $F=1 / 2$ hyperfine state are low-field seeking, except below 40 Gauss where the $\left|F=1 / 2, m_{F}=-1 / 2\right\rangle$ state is high-field seeking. The hyperfine structure therefore simplifies the optical pumping scheme: rather than truly polarize Li-6 atoms into a well-defined state $\left|F, m_{F}\right\rangle$, we can simply drive atoms out of the $F=3 / 2$ state into the $F=1 / 2$ state. The $228 \mathrm{MHz}$ hyperfine splitting of the ${ }^{2} \mathrm{~S}_{1 / 2}$ state should be sufficient for atoms in the $F=1 / 2$ state to be negligibly excited when using a laser tuned for driving transitions on a $\mathrm{D}$ line from the $F=3 / 2$ state. 

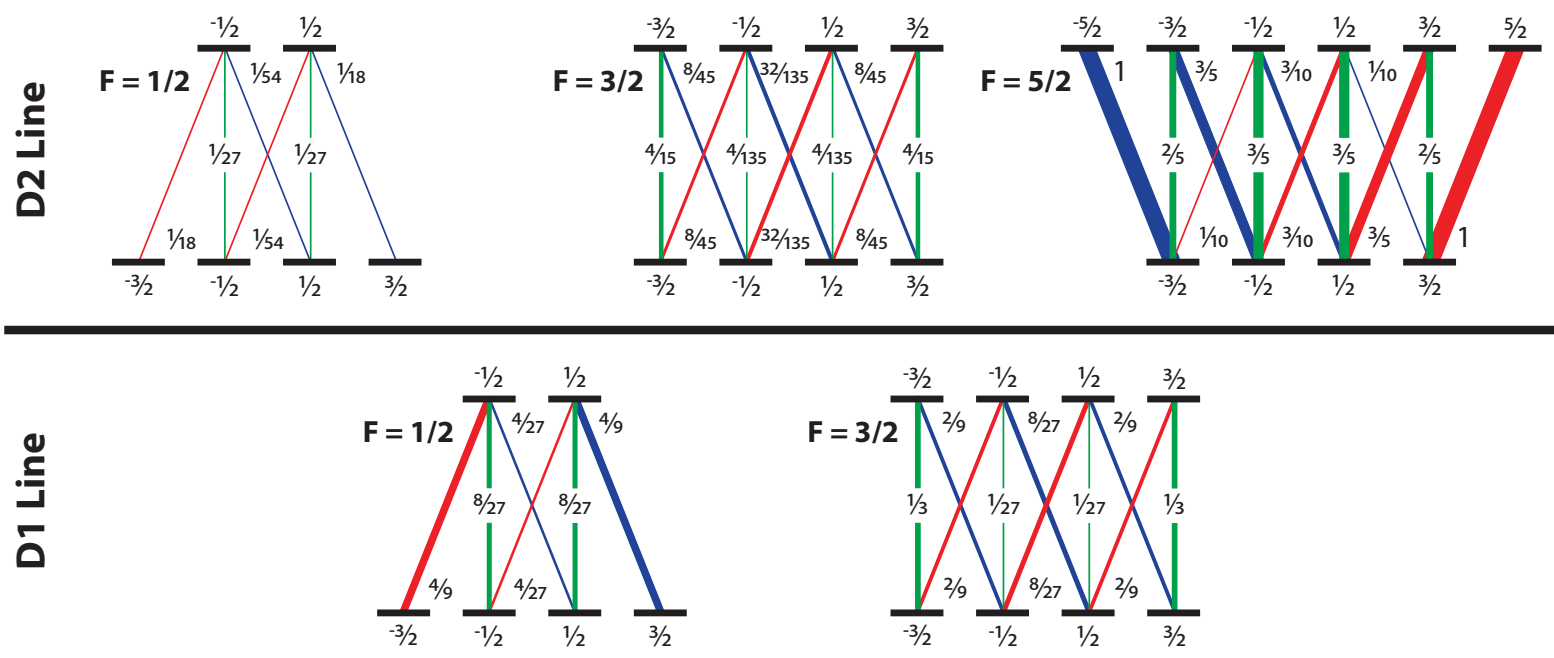

Figure 2.4: Summary of transition strengths for Li-6 D1 and D2 lines. We give relative transition strengths, with the peak strength - between the $F= \pm 3 / 2$ and $F^{\prime}= \pm 5 / 2$ states on the D2 line - being normalized to unity. This transition corresponding to the peak strength is the cycling transition that is used for laser cooling lithium.

Fig. 2.4 summarizes the relative strengths for transitions on the Li-6 D1 and D2 lines originating at the $F=3 / 2$ manifold within the ${ }^{2} \mathrm{~S}_{1 / 2}$ state. Ignoring these transition strengths, we immediately rule out using $\sigma^{+}$polarization for optical pumping on the D1 line as this will drive a fraction of Li-6 atoms into the dark (under these conditions), high-field seeking $\left|F=3 / 2, m_{F}=+3 / 2\right\rangle$ state. The strength for a particular lithium D line transition between states $\left|2^{2} \mathrm{~S}_{1 / 2}, F, m_{F}\right\rangle$ and $\left|2^{2} \mathrm{P}_{\mathrm{J}^{\prime}}, F^{\prime}, m_{F^{\prime}}\right\rangle$ is proportional to the square of the electric dipole matrix element

$$
\left(D_{F, m_{F}}^{F^{\prime}, m_{F^{\prime}}}\right)^{2}=\left|\left\langle 2^{2} \mathrm{P}_{\mathrm{J}^{\prime}}, F^{\prime}, m_{F^{\prime}}\left|d_{q}\right| 2^{2} \mathrm{~S}_{1 / 2}, F, m_{F}\right\rangle\right|^{2}
$$

where $d_{q}$ denotes the electric dipole operator, a rank 1 tensor with three quantum numbers $(q=0$ and \pm 1 , corresponding to selection rules defined by linear and circular laser polarizations). By successively applying the Wigner-Eckart theorem, (2.11) can be simplified to

$$
\begin{aligned}
\left(D_{F, m_{F}}^{F^{\prime}, m_{F^{\prime}}}\right)^{2}= & 2\left(J^{\prime}+1\right)(2 F+1)\left(2 F^{\prime}+1\right) \\
& \times\left\{\begin{array}{ccc}
1 & 0 & 1 \\
1 / 2 & J^{\prime} & 1 / 2
\end{array}\right\}^{2}\left\{\begin{array}{ccc}
J^{\prime} & 1 / 2 & 1 \\
F & F^{\prime} & 1
\end{array}\right\}^{2}\left(\begin{array}{ccc}
F^{\prime} & 1 & F \\
-m_{F^{\prime}} & q & m_{F}
\end{array}\right)^{2} \\
& \times\left|\left\langle 2^{2} P\|d\| 2^{2} S\right\rangle\right|^{2}
\end{aligned}
$$


where the terms on the second line denote $3-\mathrm{j}$ and 6 -j symbols accordingly and the final term corresponds to a reduced matrix element that is determined by the fine structure for the atom [46].

As shown in Fig. 2.4, the largest transition strength among the D lines (originating in the $F=3 / 2$ state) corresponds to the transitions between the $\mid F=3 / 2, m_{F}=$ $\pm 3 / 2\rangle$ and $\left|F^{\prime}=5 / 2, m_{F}= \pm 5 / 2\right\rangle$ states. In fact, using proper laser polarization these are cycling transitions that correspond to those used for laser cooling lithium $[47,48]$. Regardless of laser polarization, transitions between the $F=3 / 2$ and $F^{\prime}=5 / 2$ manifolds - which cannot decay to the $F=1 / 2$ state - are the strongest among all transitions on the D2 line. To most efficiently drive atoms into the $F=1 / 2$ state, we therefore operate on the D1 line. In particular, we anticipate optimal Li-6 suppression when using $\sigma^{-}$polarization in order to preferentially drive any atoms remaining in the $F=3 / 2$ manifold towards the $\left|F=3 / 2, m_{F}=-3 / 2\right\rangle$ state (which is high-field seeking). Working on the D1 line also circumvents the possibility for driving Li-7 atoms on the D2 line (with this line being atypically close to the Li-6 D1 line).

Summing (2.12) over all transitions for a given line yields the Einstein A coefficient for that line. In fact, the resulting sum $D^{2}$ over excited states $\left|F^{\prime}, m_{F^{\prime}}\right\rangle$ that couple to a given ground state $\left|F, m_{F}\right\rangle$ is independent of $F^{\prime}$ and $m_{F^{\prime}}$ and identical for all $F$ corresponding to a certain $J$. In particular, for the D1 line

$$
A_{D 1}=\frac{\omega_{D 1}^{3}}{3 \pi \epsilon_{0} \hbar c^{3}} D_{D 1}^{2}
$$

where $\omega_{D 1}$ is the absolute (angular) transition frequency between the $2^{2} \mathrm{~S}_{1 / 2}$ and $2^{2} \mathrm{P}_{1 / 2}$ states, $\epsilon_{0}$ is the permittivity of free space, and $c$ denotes the speed of light [49]. Measured decay rates therefore provide values for the reduced matrix elements in (2.12). The lifetime for the $2^{2} \mathrm{P}_{1 / 2}$ state (and also the $2^{2} \mathrm{P}_{3 / 2}$ state interestingly) - corresponding to the inverse of the Einstein A coefficient - is 27.102 ns, and thus the natural linewidth for the D1 line is $5.872 \mathrm{MHz}$ [50]. The large linewidth for the lithium D lines simplifies optical pumping by relaxing demands on laser linewidth and concomitantly allowing us to implement a simple scheme for effectively broadening the laser spectrum. 
In practice, to maximize the transition rate on the D1 line we try to maximize the area of the pumping beam over which the on-resonance saturation parameter $s_{0}$ satisfies

$$
s_{0} \equiv 2|\Omega|^{2} / A_{D 1}^{2}=I / I_{s a t} \gg 1
$$

where $\Omega$ is the Rabi frequency for the transition (given by the dipole matrix element), $I$ denotes the laser intensity, and

$$
I_{s a t} \equiv \frac{2 \pi^{2} \hbar c A_{D 1}}{3 \lambda_{D 1}^{3}}
$$

is the saturation intensity for the transition $[49,51]$. That is, we maximize the beam width (and thus interaction time with the atomic beam) over which the intensity is sufficiently high for saturating the atomic population in the ${ }^{2} \mathrm{P}_{1 / 2}$ state. For the Li-6 D1 line (D2 line) the saturation intensity is $7.59 \mathrm{~mW} / \mathrm{cm}^{2}\left(2.54 \mathrm{~mW} / \mathrm{cm}^{2}\right)$. By maximizing the saturation parameter, we likewise broaden the effective spectrum for the pumping beam by enhancing the power-broadened linewidth for the transition.

While we extensively simulated the performance of our magnetic field gradient, we opted to mostly optimize Li-6 pumping into the $F=1 / 2$ state in an empirical manner. Other work has provided thorough theoretical treatment of optical pumping $[46,52,53]$, with certain work particularly addressing (theoretically and empirically) the optical pumping of lithium $[54,55]$. For instance, Gillott et al. investigated the attainable polarization of Li-7 atoms in a supersonic beam into the $\left|F=2, m_{F}= \pm 2\right\rangle$ ground state. By numerically integrating semi-classical rate equations for atomic populations, they generated curves predicting changes in atomic populations as atoms traverse a laser beam. As expected, atomic polarization depends on factors including laser intensity/polarization and interaction time.

In contrast to true optical pumping into a well defined state $\left|F, m_{F}\right\rangle$, the efficiency for hyperfine pumping Li- 6 atoms into the $F=1 / 2$ ground state should be less polarization sensitive. In this case, the hyperfine splitting of the ground state - rather than a selection rule - makes the $F=1 / 2$ state "dark". Radiation trapping will likely worsen our atomic polarization when working at our highest source temperatures. A figure-of-merit for the impact of radiation trapping on de-polarization is given by the 
product of atomic density and atomic beam diameter where the optical pumping beam intersects the atomic beam $[56]^{3}$. Beyond $10^{11}$ atoms $/ \mathrm{cm}^{2}$, polarization rapidly worsens for effusive beams.

We lastly note that the recoil velocity for the D1 line is close to $10 \mathrm{~cm} / \mathrm{s}$. Optical pumping Li-6 atoms into the $F=1 / 2$ ground state can require several photons per atom. As the magnetic field gradient selects a narrow transverse velocity distribution among atomic trajectories, photon recoil can alter atomic velocity components perpendicular to the field gradient by a non-negligible amount. For experimental simplicity (relating to both our optical layout and minimizing Doppler shifts), we direct the optical pumping beam perpendicular to the atomic beam. By initially directing the optical pumping beam toward the magnets, we exert radiation pressure onto Li-6 that should contribute toward ensuring that Li-6 atoms cannot reach beyond the magnet barrier to the collection plane.

\subsection{Magnetic Guide}

\subsubsection{Halbach Arrays and One-Sided Flux}

We produce the magnetic field gradient for achieving Li-7 enrichment using rareearth permanent magnets. Since the 1980s planar arrangements of permanent magnets have been used for generating synchrotron radiation from electron storage rings [57]. These devices - called either wigglers or undulators - consist of a pair of linear, parallel sequences of permanent magnets (each called a Halbach array) as exemplified in Fig. 2.5. The height of the magnets (out of the plane of the page) typically far exceeds the gap between the sequences. The simplest designs use magnets that have rectangular cross-sections with the magnetization for every magnet oriented along an edge of the cross-section. By rotating the magnetization direction between adjacent magnets in a periodic manner (using opposite rotation directions for the two sequences), the sequences suppress the field component along sequences (x-component) at the center of the gap while producing sinusoidal variation in the y-component (with large amplitude) whose

\footnotetext{
${ }^{3}$ Peterson et. al particularly theoretically investigate an optical pumping beam transverse to an alkali atomic beam.
} 
A

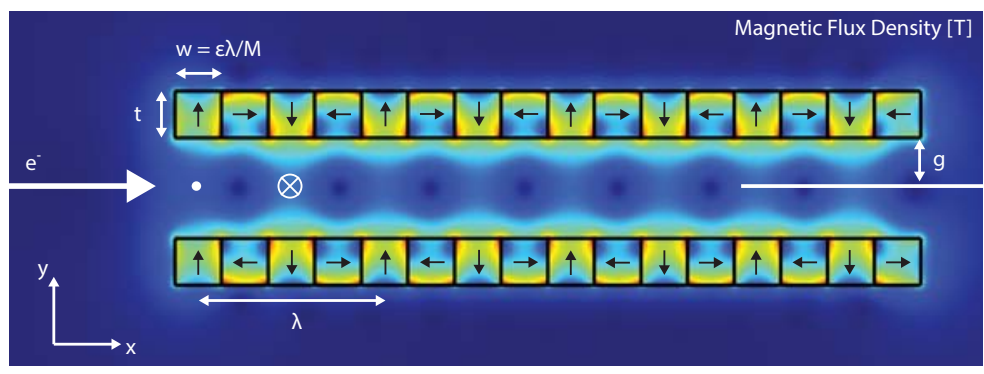

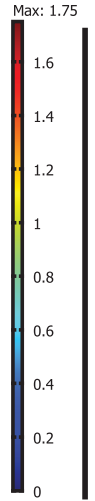

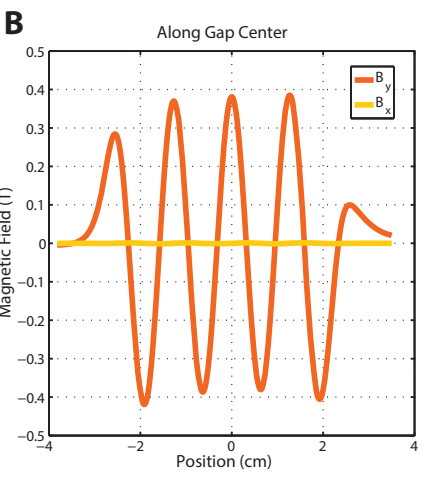

Figure 2.5: Overview of undulators used for generating synchrotron radiation. (A) Undulators consist of a pair of linear magnet sequences (spaced by $2 g$ ). The magnetization vector rotates in a periodic manner (with periodicity $\lambda$ ) between adjacent magnets (of thickness $t$ and width $w=\epsilon \lambda / M$ with $0 \leq \epsilon \leq 1$ ) along both sequences, with the rotation direction being opposite between the pair of sequences. (B) This configuration suppresses the $\mathrm{x}$-component of the magnetic field between the sequences while causing the y-component to oscillate along the length. Incident electrons deflect into and out of the page, emitting radiation along their propagation direction.

periodicity is determined by the spacing between magnets with identical magnetization.

This combination of Halbach arrays causes incident electrons to oscillate along the direction of the magnet height and emit radiation along the beam direction. Characteristics including the amplitude and periodicity of the magnetic field, divergence of the electron beam, and electron energies determine spectral properties of the radiation produced ${ }^{4}$. While other technologies like superconducting magnets have been used in wiggler/undulator design, rare-earth permanent magnets - such as samarium-cobalt $(\mathrm{SmCo})$ and neodymium-iron-boron $(\mathrm{NdFeB})$ - present a series of favorable properties. In contrast to electromagnets, the peak field produced along the gap remains constant when scaling all linear dimensions. The small sizes available for permanent magnets therefore can enable shorter period lengths that in turn yield larger peak fields. Certain magnetic materials like SmCo and $\mathrm{NdFeB}$ are highly anisotropic, yielding large remnant fields - close to $1.5 \mathrm{~T}$ for the highest grade NdFeB magnets (N52) - along an easy axis

\footnotetext{
${ }^{4}$ The definition of the magnet sequences as either undulators or wigglers depends on features of the radiation.
} 


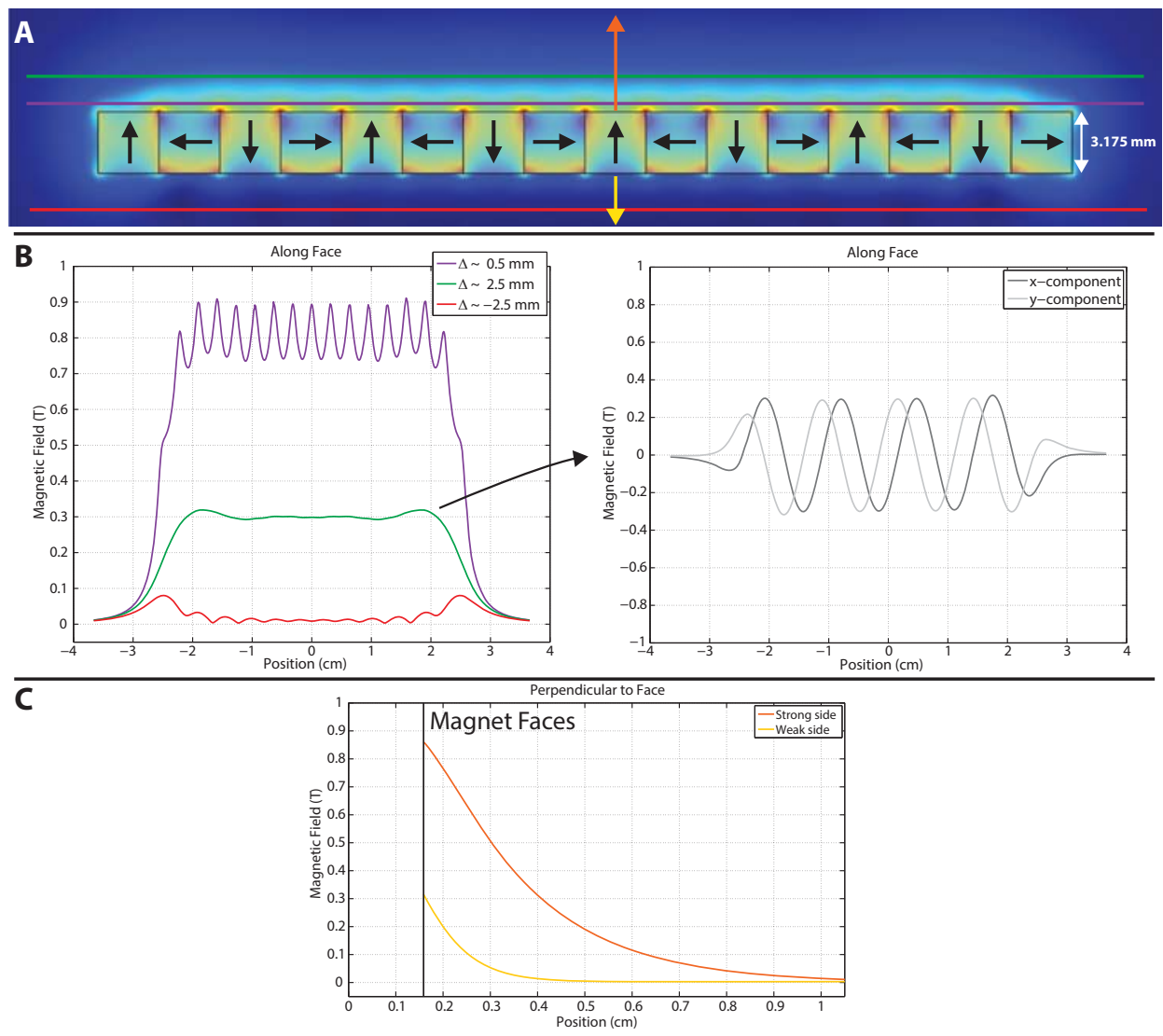

Figure 2.6: Characteristics of Halbach arrays. (A) Rotating the magnetization direction between adjacent permanent magnets amplifies the magnetic field on one face of the array while attenuating the field on the opposite face. (B) While the field components are shifted in phase by $\pi / 2$ (causing the field vector to rotate with periodicity given by that of the magnetization vectors), the magnetic flux density is close to uniform along the array with the uniformity improving for distances $\Delta$ further away from the magnets. (C) The field decays over a distance on the order of the magnet thickness.

with essentially zero remnant field perpendicular to this axis ${ }^{5}$. Beyond just producing large fields in the Halbach configuration, these features allow the field along the gap of wigglers/undulators to be described analytically [57].

A key feature of these undulators is that the magnetic flux is confined to the interior faces of the Halbach arrays. Mallinson first showed that certain magnetization patterns in various structures produce "one-sided fluxes" [58]. The planar Halbach

\footnotetext{
${ }^{5}$ For example, refer to information provided by K\&J Magnetics, Inc. See http://www.kjmagnetics.com.
} 
array described above corresponds to one of the simplest realizations of a one-sided flux. Fig. 2.6 outlines several features of a single array (having four magnets per period). As evident by the magnetization vectors, field lines combine to attenuate/amplify the magnetic field on the bottom/top face of the array. In contrast to the pair of adjacent arrays in Fig. 2.5, the x-component of the magnetic flux density is not suppressed on the side of the magnets where the field is amplified. Instead, the $\mathrm{x}$ - and $\mathrm{y}$-components appear identical, but shifted in phase by $\pi / 2$. As a result, while the field direction rotates with a periodicity given by the magnet layout, the magnetic flux density is nearly uniform across the face. The flux density decays roughly exponentially upon moving away from either face of the array over a distance comparable to the magnet thickness.

Subsequent work discussed using permanent magnets for making multipole magnets [59]. These configurations consist of a closed Halbach array arranged around a cylinder, producing large magnetic flux density across the bore. In fact, a previous proposal for isotope separation that similarly combined optical pumping and a magnetic field gradient suggested using four permanent magnets for producing a quadrupole field in order to accomplish Li-6 enrichment $[60,61]$. By utilizing bright and directional supersonic beams, notably the Even-Lavie valve [62], this work suggested the ability to collect close to $10^{16}$ Li- 6 atoms per second. This work similarly proposed to suppress Li-7 throughput beyond the quadrupole by pumping Li-7 accordingly into a high-field seeking state. While being an impressive number for a quadrupole with $1.5 \mathrm{~cm}$ bore, the axially-symmetric geometry for the magnetic field gradient does not lend itself toward easily producing larger quantities. Scaling throughput will require apparatus in parallel, with every apparatus including a supersonic valve, ablation laser for entraining lithium atoms into a supersonic beam, and large turbomolecular pumps for managing the large gas load introduced into the apparatus by a noble carrier gas ${ }^{6}$.

A straight quadrupole without any obstruction for high-field seeking Li-7 atoms will not be able to perfectly enrich Li-6, although the earlier work predicts that Li-6

\footnotetext{
${ }^{6}$ In addition, given recent personal experience working toward entraining lithium into supersonic beams, $10 \%$ entrainment efficiency appears optimistic. Moreover, operating the (pulsed) Even-Lavie valve at a $1 \mathrm{kHz}$ repetition rate poses significant challenges due to the massive gas load into the vacuum chamber.
} 
enrichment to $95 \%$ (corresponding to an enrichment factor close to 240) should be feasible using a $50 \mathrm{~cm}$ long quadrupole. Another possibility - actually demonstrated using thermal beams for basic research - that might yield higher enrichment includes bending the quadrupole over its length in order to suppress line-of-sight between the supersonic valve and the exit aperture for the quadrupole [63,64]. A more serious detriment to the proposal, however, arises from the possibility of material clogging the bore of the quadrupole after prolonged use. Over the course of operation, throughput will continuously worsen as lithium condenses along the bore and reduces its effective aperture.

\subsubsection{Curved Halbach Array}

Planar Halbach arrays present a more viable option for scaling throughput in a commercially feasible manner. While supersonic beams produce bright and highly directional atomic beams, thermal beams more readily provide higher total flux albeit into a substantially larger solid angle. Planar arrays can capture material over a large fraction of this solid angle. For example, suppose that the exit aperture for a thermal source lies in the zy-plane for a Cartesian coordinate system with its center positioned at the origin. Choosing the aperture to have a narrow extent along the y-direction will limit the azimuthal distribution for atomic trajectories incident on an azimuthal extent on a hemisphere of a given radius that is centered about the origin. By thus positioning a planar array at a suitable distance in front of the aperture with the plane defining its end normal to a segment drawn in the xy-plane outward from the center of the source aperture, the array should sample a narrow subset of the azimuthal distribution emitted by the source.

By extending the height of the magnets, the array can sample a large fraction of the polar angle distribution for trajectories originating on the source aperture. The exact height necessary for capturing this entire distribution will depend on both the height of the source aperture and the distance between the source and the array. Similarly to a straight magnetic quadrupole, the attainable purity will be fundamentally limited by the planar geometry (as atoms with certain incidence angles and sufficiently large kinetic energy will not be deflected by enough to prevent them from reaching beyond the plane). 
The purity can be improved by appending an aperture to the front of the Halbach array that further restricts the azimuthal distribution that samples the magnets. Reducing this aperture width, however, will improve purity at the expense of throughput.

We can alternatively drastically improve the degree of enrichment by slightly curving the Halbach array over its length. By choosing the aperture width to match the lateral displacement of the array along its length, no atoms incident upon the aperture should have line-of-sight to beyond the panel (as long as the source is positioned properly). In this configuration, only low-field seeking atoms that reflect away from the magnets can reach the plane beyond the array. In the context of this work, assuming that optical pumping substantially populates Li- 6 atoms in the $F=1 / 2$ state, the enrichment factor for Li-7 should be very high. By adjusting the curvature and length of the array, we can balance the efficiency for guiding low-field seeking atoms and practical considerations (including magnet cost and apparatus extent). The guiding efficiency for a given geometry will depend on the curvature and thermal energy that atoms acquire in the source: the array will not reflect trajectories whose kinetic energies corresponding to velocity components perpendicular to the array exceed the peak magnetic potential produced at the array surface.

By similarly positioning identical arrays about the source, we can collect a larger fraction of source material. The ultimate fraction of the solid angle that the magnets sample will be limited by the solid angle subtended by the ends of all of the arrays to the source. In principle, however, this sampling fraction could reach beyond $50 \%$ by suitably choosing the array geometries. The one-sided flux produced by Halbach arrays mitigates concern about an adjacent panel worsening the performance of a given panel (although residual fields could slightly contaminate purity) [27]. We can enhance flux without compromising efficiency by extending the source height (in order to increase its area). Conversely, we can improve efficiency without sacrificing flux by reducing the source temperature and increasing the source height.

While in this work we use a single array for suppressing throughput of one isotope in order to enrich a second isotope, the exact scheme can be modified depending on the isotope of interest. By more generally using pairs of guides in series (with ideally multiple 

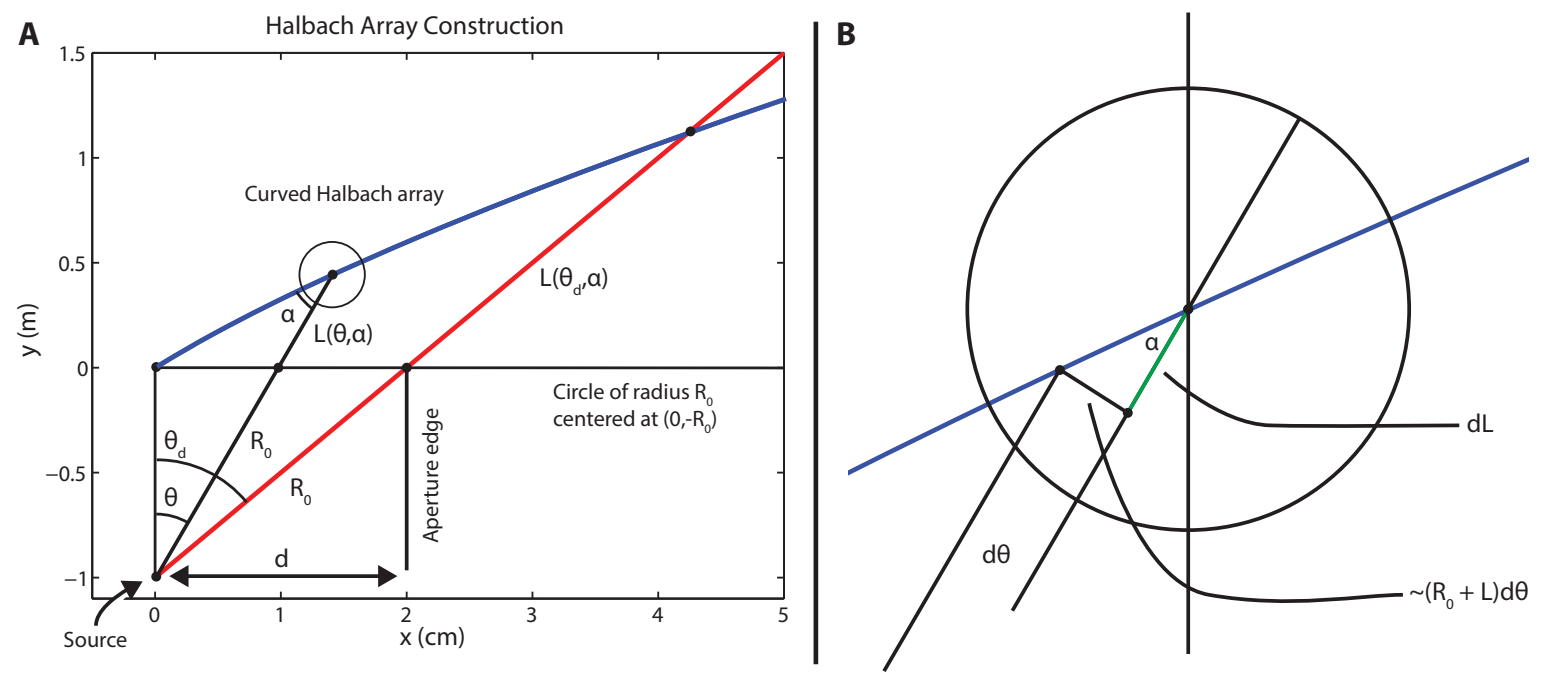

Figure 2.7: Outline for guide geometry. (A) All trajectories originating at a point source positioned accordingly should impinge on the curve $L(\theta, \alpha)$ defining the Halbach array at a fixed angle $\alpha$. An aperture at the guide entrance governs the maximum angle $\left(\theta_{d}\right.$ as measured in the coordinate system) incident on the magnets, and thus the minimum length for the guide. (B) Finding an analytical expression for the curve proceeds by deriving a relationship between differential elements $\mathrm{d} \theta$ and $\mathrm{d} L$ and integrating.

stages of optical pumping), we can more generally enrich any isotope among arbitrarily many isotopes. We discuss this layout in more detail in a later chapter.

Bruce Klappauf designed the guide that we used for this proof-of-principle work and simulated its performance. The guide originates on a circle of radius $R_{0}$ centered about the source. Assuming a point source at the center of this circle, we configure the curvature of the guide such that all trajectories impinge on the magnet surface at the same angle $\alpha$. As outlined in Fig. 2.7, in order to satisfy this condition the curve parameterized using polar coordinates $(\theta, L)$ with the origin offset to $(x, y)=\left(0,-R_{0}\right)$ and $\theta$ measured from the $\mathrm{y}$-axis - must satisfy

$$
\tan \alpha=\frac{\left(R_{0}+L\right) \mathrm{d} \theta}{\mathrm{d} L}
$$

where $\mathrm{d} \theta$ and $\mathrm{d} L$ denote differential changes in polar coordinates upon advancing along the curve by an infinitesimal arc length $\mathrm{d} s$. By rearranging 2.16 and integrating over the limits for $L$ and $\theta$, we obtain the functional form for the guide:

$$
L(\theta, \alpha)=R_{0}\left(\exp \left[\frac{\theta}{\tan \alpha}\right]-1\right)
$$



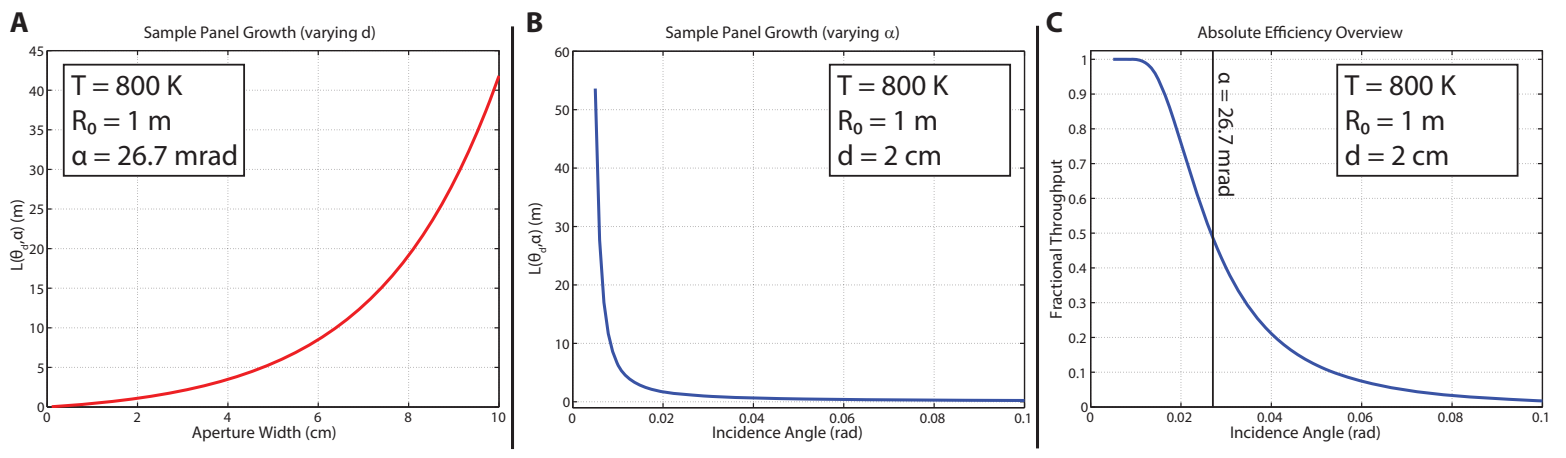

Figure 2.8: Guide geometry considerations. (A) The minimum guide length $L\left(\theta_{d}, \alpha\right)$ increases exponentially upon widening the guide aperture. (B) In contrast, the minimum guide length plummets rapidly for a steeper incidence angle. (C) Increasing the incidence angle, however, reduces the fractional throughput reaching beyond the magnets.

An aperture at the guide entrance determines the maximum angle $\theta_{d}$ among trajectories originating at the source that the guide must sample. This angle (combined with $\alpha$ ) in turn configures the minimum guide length in order to ensure no line-of-sight between the source and the plane beyond the magnets.

Fig. 2.8(A) shows the exponential growth - evident from 2.17 - of the minimum guide length as a function of this aperture width. The design for the guide should enable all trajectories up to a threshold speed to be deflected by the magnets (assuming a point source), with this threshold corresponding to when the velocity component perpendicular to the curve exceeds the magnetic potential produced by the magnets ${ }^{7}$. In particular, this speed $v_{t}$ is given by

$$
v_{t}=\sqrt{\left(2 \mu_{B} B_{0} / m\right)} / \sin \alpha
$$

where $B_{0}$ should be close to the remnant flux density for the permanent magnets. Fig. $2.8(\mathrm{C})$ shows the fraction of trajectories emitted by a source operating at $800 \mathrm{~K}$ - using realistic aperture width and source-to-guide distance - that the guide should deflect over a range of values for $\alpha$. While the lowest $\alpha$ values yield the best absolute efficiencies for guiding, these shallow angles require prohibitively long guides (as shown in Fig. 2.8(B)).

\footnotetext{
${ }^{7}$ By design, this velocity component is the same for all trajectories with a particular speed in the case of a point source.
} 


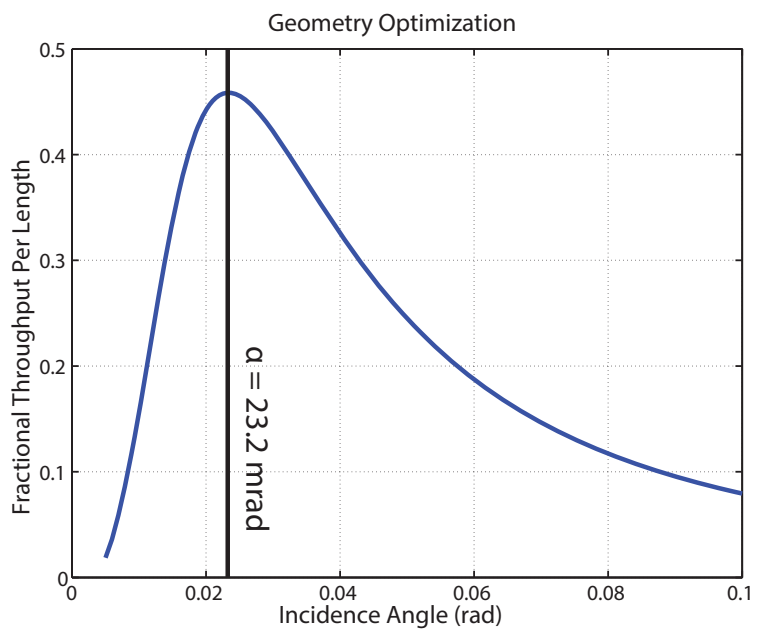

Figure 2.9: Optimizing guide characteristics. Fixing $d=2 \mathrm{~cm}$ and $R_{0}=1 \mathrm{~m}$, the ratio between fractional throughput and guide length as a function of $\alpha$ yields a maximum. The conditions corresponding to the maximum present a compromise between performance and practicality.

Beyond $\alpha \sim 20 \mathrm{mrad}$, the necessary guide length is less than $2 \mathrm{~m}$ for $R_{0}=1 \mathrm{~m}$ and $d=2 \mathrm{~cm}$. Fixing this value for $R_{0}$, we compromise building a compact apparatus while working with manageable atomic densities for the optical pumping of Li-6 over the temperature range of interest. Likewise, choosing $d=2 \mathrm{~cm}$ with $R_{0}=1 \mathrm{~m}$ maintains a reasonable Doppler spread (between 50-100 MHz at the source temperatures we consider for the lithium D lines) for simplifying optical pumping. While efficiency worsens for increasing $\alpha$, Fig. 2.8(C) indicates that the guiding efficiency remains higher than $50 \%$ (for a point source) at $\alpha=20 \mathrm{mrad}$. In fact, figure 2.9 shows that the ratio of fractional throughput to guide length (for constant $R_{0}$ and $d$ ) yields a maximum at $\alpha=23.2 \mathrm{mrad}$. We use this ratio as a figure-of-merit for simultaneously optimizing the guide length and incidence angle. For the guide that we ultimately constructed, we chose $\alpha=20$ mrad while using a (adjustable) $1.5 \mathrm{~cm}$ wide aperture. We extended the guide length to close to $1.5 \mathrm{~m}$ for absolutely ensuring no line-of-sight between the source (of finite width in the apparatus) and the plane beyond the magnets. We also slightly reduced the sourceto-guide distance. 


\subsection{Numerical Simulations}

Bruce Klappauf incorporated this curved Halbach array (among alternative designs) into a Python-based numerical simulation for examining the performance or our apparatus. This simulation numerically integrated classical equations of motion derived using the interaction energy given by (2.3). The simulation generated initial conditions by partitioning the width of a source aperture (chosen to be $1 \mathrm{~mm}$ in most cases), incidence angles upon the guide aperture, and relevant speeds among the distribution corresponding to a given source temperature. Trajectories originate on the source aperture and propagate freely (according to their angles) to the magnet entrance prior to sampling the magnetic field. Finite-element analysis generated the magnetic field produced by a given permanent magnet array over the relevant area that trajectories might sample ${ }^{8}$. To mimic the actual guide that we constructed, the Python simulation produces directives for COMSOL to generate a planar model consisting of segments of permanent magnets whose ends lie on the curve given by (2.17) (for a set of geometric parameters). To place these segments accordingly, the simulation redefines 2.17 using Cartesian coordinates:

$$
x=\left(R_{0}+L\right) \sin \theta \text { and } y=\left(R_{0}+L\right) \cos \theta-R_{0}
$$

Given the planar field configuration, the simulation considers only two-dimensional trajectories. The guide surface and aperture define boundaries for trajectories. We also impose a fictional boundary corresponding to a fixed distance away from $x=0$ (see Fig. 2.7). This constraint prevents trajectories from traversing beyond the spatial extent for the magnetic fields that we extract from COMSOL.

Fig. 2.10 exemplifies an initial phase space for low-field seeking Li-6 trajectories incident on the guide aperture. These trajectories correspond to a simulation that best reproduces observables that we measure with our proof-of-principle apparatus. This plot shows angles of incidence (corresponding to $\theta$ in Fig. 2.7) onto the magnet aperture rather than just initial positions along the source aperture. Blue trajectories denote

\footnotetext{
${ }^{8}$ We particularly use COMSOL Multiphysics. See http://www.comsol.com.
} 


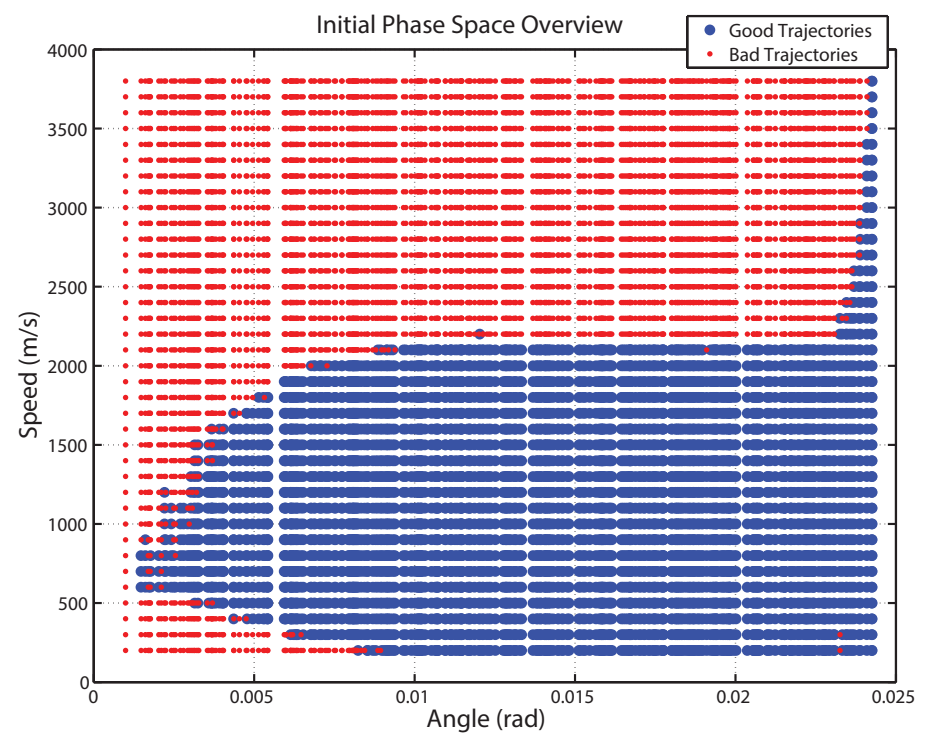

Figure 2.10: Overview of initial phase space for incidence angles and speeds. Blue points show trajectories reaching beyond the magnets, while red points denote those that encounter a simulation constraint.

those that ultimately reach a plane beyond the magnets, while red trajectories correspond to those that encounter one of the constraints outlined above. As anticipated, we observe a cut-off speed at close to $2200 \mathrm{~m} / \mathrm{s}$ beyond which almost every trajectory strikes the magnets. The simulation surprisingly indicates higher guiding efficiency for trajectories with initial speeds between 500 and $1000 \mathrm{~m} / \mathrm{s}$ than for those with lesser speeds. The lowest speed atoms incident on the guide aperture at the shallowest angles (corresponding non-intuitively to larger angles in Fig. 2.10) deflect at steep angles that ultimately terminate on the fictional boundary. In practice, these atoms will most likely not be collected due to the presence of an adjacent guide.

Fig. 2.11(A) shows the terminal speeds and lateral positions for all of the trajectories. Those trajectories with speeds below threshold that do not reach beyond the magnets impinge on the magnets immediately following the magnet aperture. A small set of trajectories that successfully pass the magnets overlap in lateral position with those trajectories encountering the fictional constraint. This apparent contradiction of the constraint is an artifact of allowing the atoms to propagate a short distance upon traversing the final magnet (for the sake of mimicing the resulting distribution at a de- 

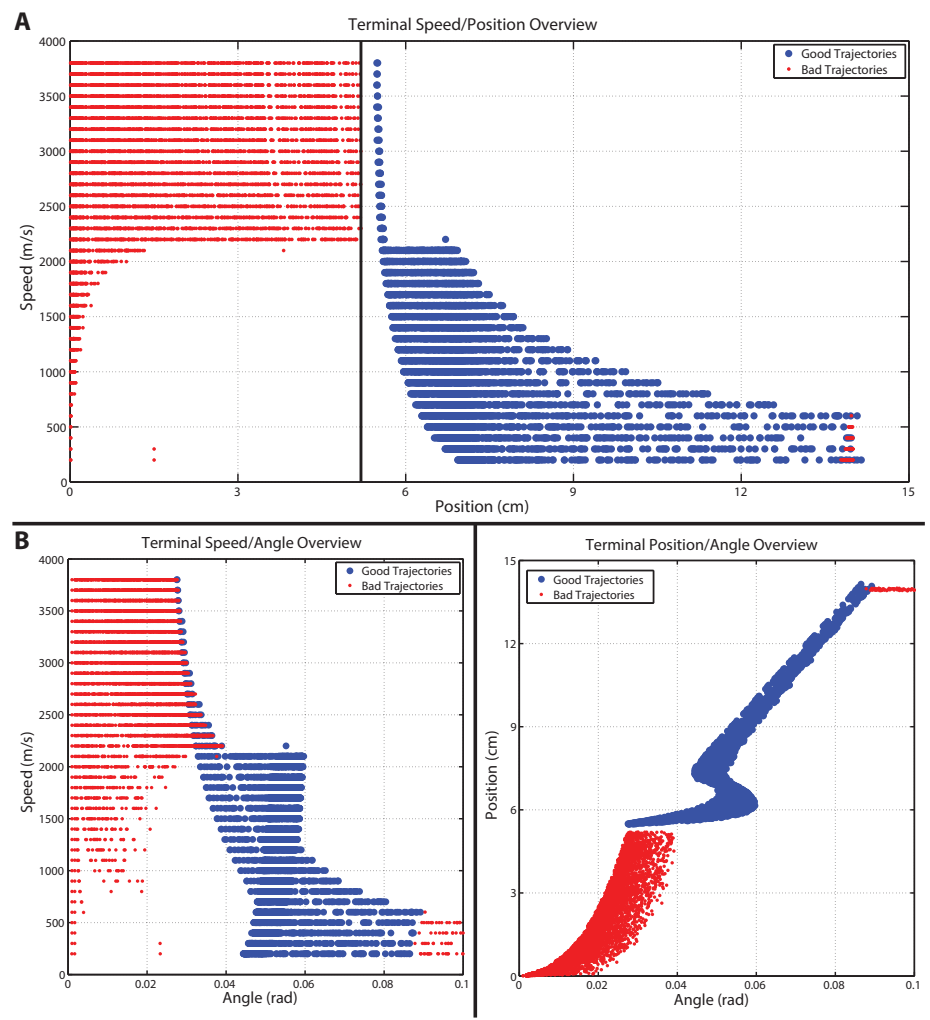

Figure 2.11: Overview of terminal phase space. (A) Phase space plot for terminal positions and speeds of all trajectories. Free-flight between the last magnet and a collection plane yields the gap between good and bad trajectories. (B) Additional phase space plots comparing terminal angles/speeds and angles/positions. 


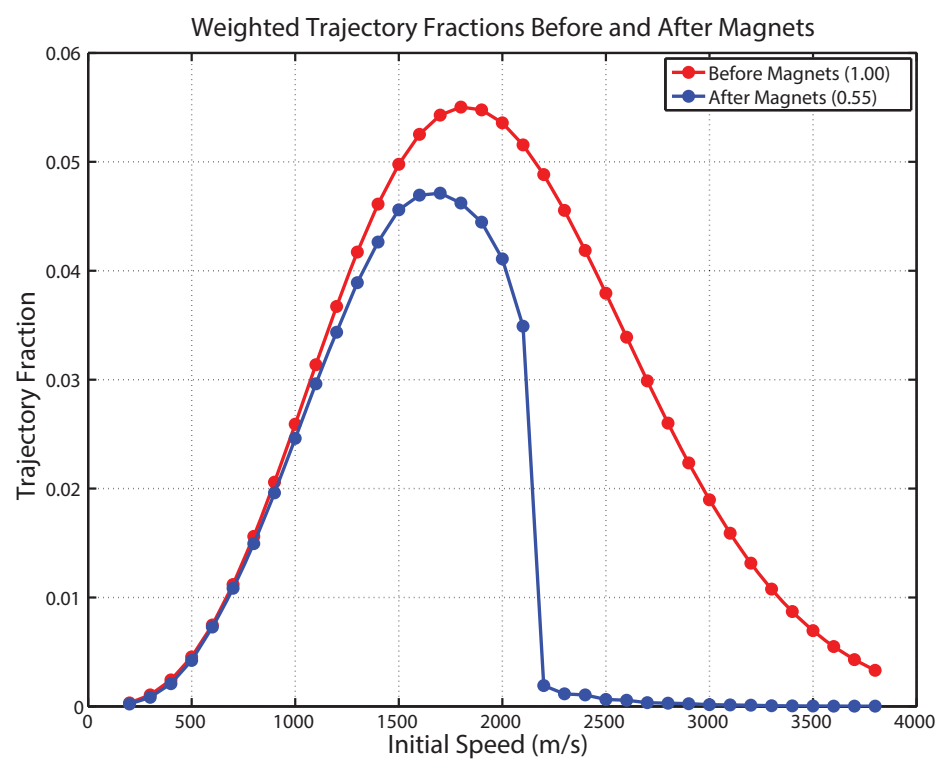

Figure 2.12: Trajectory fractions for given speeds before (red) and after (blue) magnet panel. In both cases, we weight the number of trajectories at a given speed by a factor given by the Maxwell-Boltzmann distribution.

tector location). The gap separating the locations of good and bad trajectories similarly results from this region of free-flight. Fig. 2.11(B) instead maps outgoing angles and terminal speeds. For speeds beyond the cut-off, some trajectories - with outgoing angles between 3 and $4 \mathrm{mrad}$ - reach beyond the magnets, while others strike the magnets. The ultimate fate for trajectories in this region of the terminal angle-speed phase space likely lies in the location of a given trajectory along the source aperture. Fig. 2.11(C) shows that these trajectories with common outgoing angles collect near the last magnet. Certain trajectories starting left-of-center on the source aperture (i.e. opposite the bend direction) encounter the magnets closer to the guide aperture than counterparts with identical incidence angles that originate to the right-of-center. This class of trajectories will be slightly deflected away from the surface, but at an angle shallow enough to strike the magnets further along the guide length.

Fig. 2.12 compares the speed distributions for all trajectories and just those reaching beyond the magnets. We weight the number of trajectories for every speed accordingly in order to yield a distribution that reflects Maxwell-Boltzmann statistics (using an $800 \mathrm{~K}$ source in this case). Similarly weighting just the good trajectory 

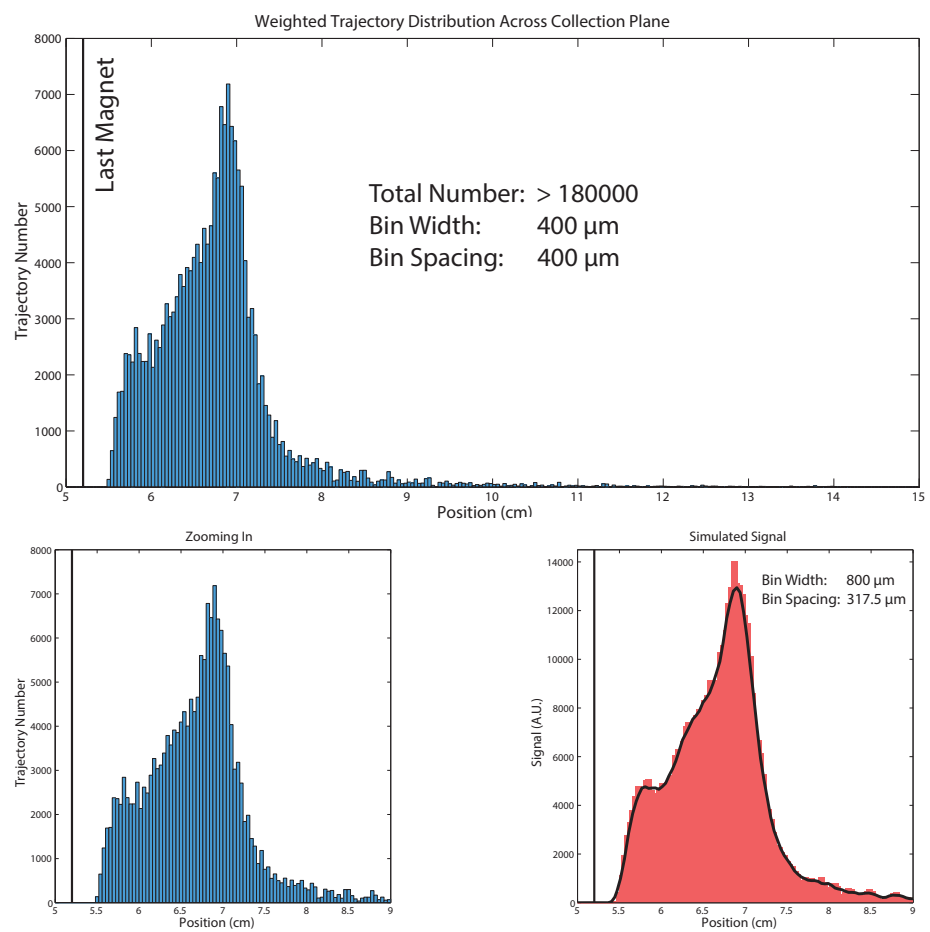

Figure 2.13: Distribution for (weighted) trajectories reaching beyond magnets. The histogram corresponds to a superposition of histograms with each containing all (good) trajectories for a given initial speed. Trajectory numbers within a particular histogram are weighted by a factor that is proportional to the probability given by the MaxwellBoltmann distribution at $800 \mathrm{~K}$. Lower right histogram adjusts the bin width and spacing in an effort to replicate a trace produced using a surface-ionization detector. We applied smoothing to the black outline in order to suppress effects relating to limited statistics.

fractions for given speeds by Maxwell-Boltzmann factors, the resulting distribution again resembles a Maxwell-Boltzmann distribution up to the cut-off speed. Integrating this discrete distribution indicates that over $50 \%$ of trajectories among a realistic initial distribution should be collected. As only low-field seeking atoms were considered in this simulation, the total efficiency (at $800 \mathrm{~K}$ ) should be close to $25 \%$ for atoms of a given isotope. While this simulation only considered Li-6 trajectories, behavior for Li-7 should be similar.

Fig. 2.13 shows a histogram that bins good trajectories in positions along the plane beyond the magnets. To obtain this histogram, we individually produced histograms for trajectories of every initial speed that the simulation considered. We weighted trajectory numbers for all speeds accordingly (as in the case of Fig. 2.12) 


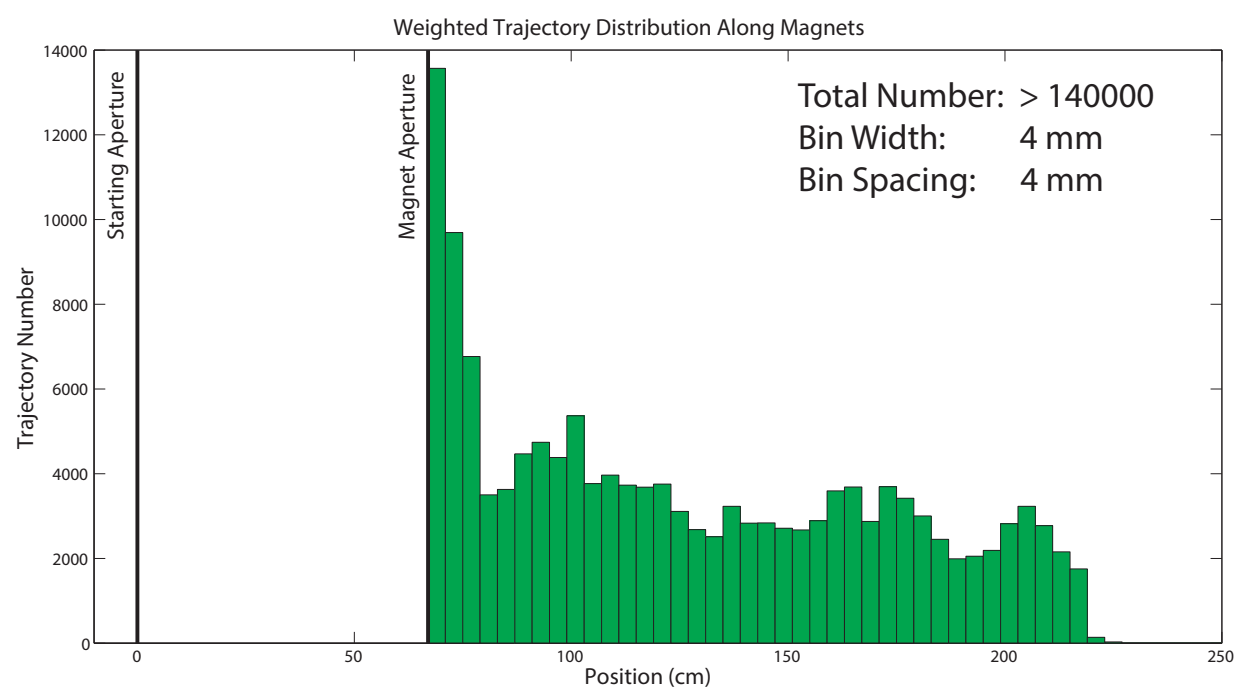

Figure 2.14: Distribution for (weighted) trajectories hitting magnets. Like Fig. 2.13, this histogram consists of a sum of weighted histograms.

then combined the resulting histograms. As indicated by Fig. 2.11, we expect a peak in the profile for the throughput with a tail that extends over several centimeters away from the magnets. By adjusting the bin width and spacing, we extract a trace that we compare to an actual measurement of the throughput that we obtained using a surfaceionization detector. In Fig. 2.14 we similarly bin the bad trajectories along the magnet length for the sake of checking for "hot-spots" where material might collect more rapidly, jeopardizing the guide performance. This distribution appears mostly uniform except for slightly higher incidence at the front of the array.

For the sake of visualizing trajectories, Fig. 2.15 shows the terminal positions for all trajectories considered by this simulation. Among the lowest speed trajectories, only a few strike the magnets, with these atoms hitting the magnets closest to the magnet aperture. By offsetting the source to the left along the x-axis, we likely could attenuate this build-up. In fact, this simulation already initiated the center of the $1 \mathrm{~mm}$ wide source aperture at $x=-1 \mathrm{~mm}$. By extending the guide length beyond the minimum length set by $\theta_{d}$, we can slightly improve the guide efficiency without sacrificing purity by offsetting the source aperture.

Figs. 2.16 and 2.17 show separate (lower trajectory number) simulations that 

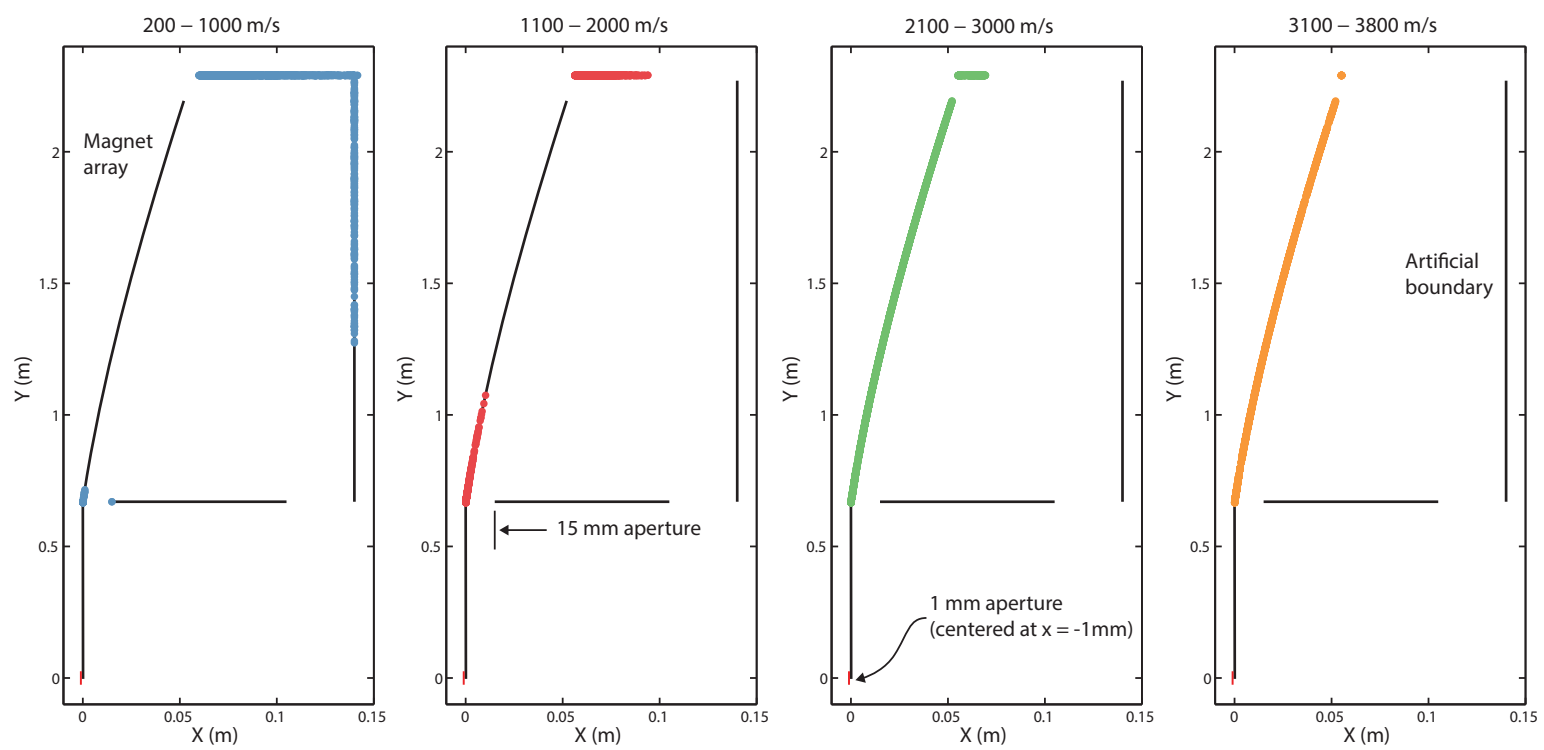

Figure 2.15: Visual summary of terminal trajectories for different speed classes. Almost none of the lowest speed trajectories $(200-1000 \mathrm{~m} / \mathrm{s})$ terminate on the guide. In fact a fraction of these trajectories acquire sufficently large outgoing angles for reaching the fictitious boundary. Larger fractions of higher speed trajectories terminate on the magnets, with almost no atoms beyond $3100 \mathrm{~m} / \mathrm{s}$ reaching beyond the guide.
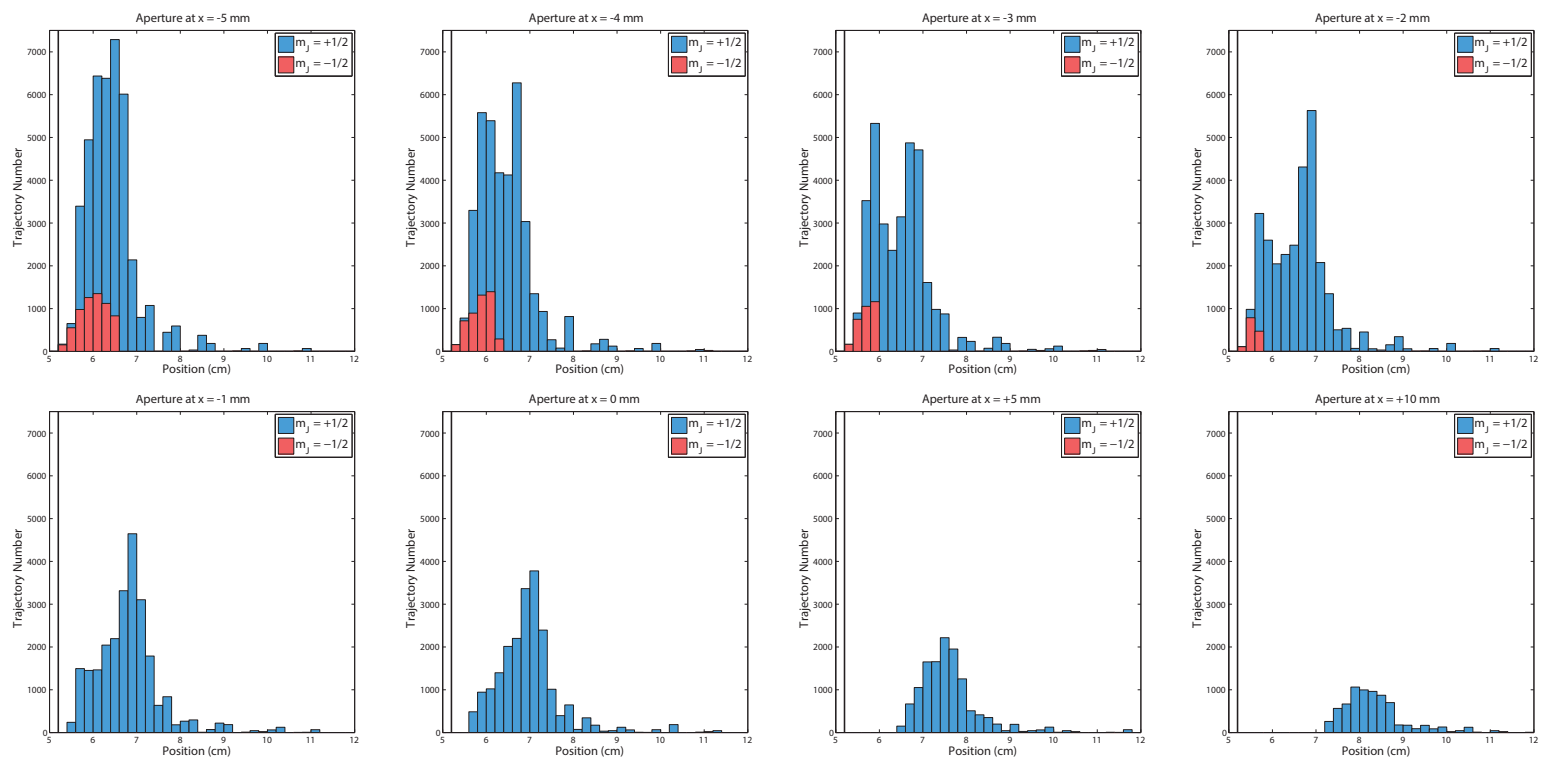

Figure 2.16: Comparing simulated throughput for $m_{J}=+1 / 2$ and $m_{J}=-1 / 2$ atoms for various oven positions. High-field seeking trajectories contaminate the throughput immediately adjacent to the last magnet upon moving the source far enough to the left. In contrast, moving the source to the right reduces the guiding efficiency for low-field seeking atoms. 


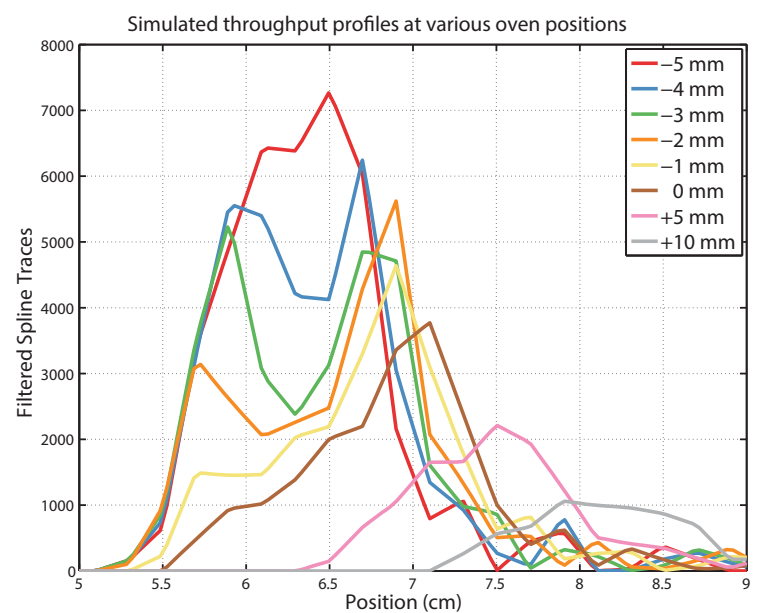

Figure 2.17: Summary of predicted throughput traces for different source positions. Moving the source aperture far enough to left ( $\mathrm{x}=-2 \mathrm{~mm}$ and beyond) introduces a second peak due to the contribution of high-field seeking trajectories passing directly from the source to the collection plane.

consider alternative source positions while sampling both low- and high-field seeking atoms. At source aperture positions beyond $x=-1 \mathrm{~mm}$, high-field seeking trajectories appear in the throughput immediately adjacent to the last magnet. Moving the aperture further to the left, more of the aperture area has line-of-sight to the collection plane. In practice, this will lead to worsening purity as these high-field seeking atoms will include Li-6 atoms that we have prepared in the $F=1 / 2$ ground state. In contrast, translating the source aperture in the positive direction reduces throughput for lowfield seeking trajectories. Velocity components perpendicular to the magnet face will be higher for these source positions. In addition, the guide aperture will reduce the number of trajectories that can sample the entire guide length. We thus identify a criterion for most efficiently enriching Li-7: we must position the source as close as possible to a position granting line-of-sight to beyond the magnets without actually enabling line-ofsight for any part of the source aperture. 


\section{Chapter 3}

\section{Experiment Overview}

\subsection{Atomic Source}

We eventually used three different sources for various measurements. For clarity, we will specify the source used for particular measurements by G1, G2, and G3 . All three sources consisted of resistively heated, stainless steel crucibles that mated to the apparatus using ConFlat (CF) flanges. The geometry of each source limited heat flow away from its reservoir, and extensive insulation prevented heat loss to the surrounding room. Type-K thermocouples measured temperatures at relevant points, and microcontroller-based feedback control maintained these temperatures to within tolerable limits.

\subsubsection{Various Iterations}

We initially adopted a spare source (G1) - shown in Fig. 3.1 - designed for another apparatus pursuing experiments derived from degenerate Fermi gases of lithium$6[47,48]$. A 1-1/3 in CF flange interfaces this oven to the apparatus. The reservoir outer diameter is $1.5 \mathrm{in}$ and its capacity is roughly $1.25 \mathrm{in}^{3}$ (equivalent to several grams of lithium metal). A tube with 0.394 in inner diameter is welded between the reservoir and the flange. The wall thickness of this tube reduces to just 0.020 in at the interface with the reservoir in order to choke off heat transfer away from the reservoir.

We loaded this source with several grams of enriched Li-6 (nominally at 95\% purity) at a time ${ }^{1}$. All enriched Li-6 material that we acquired was stored in mineral oil. We therefore rinsed the material in acetone and removed the heavily contaminated

\footnotetext{
${ }^{1}$ We acquired enriched Li-6 from Cambridge Isotope Laboratories, Inc. in $10 \mathrm{~g}$ quantities packed in mineral oil. See http://www.isotopes.com.
} 

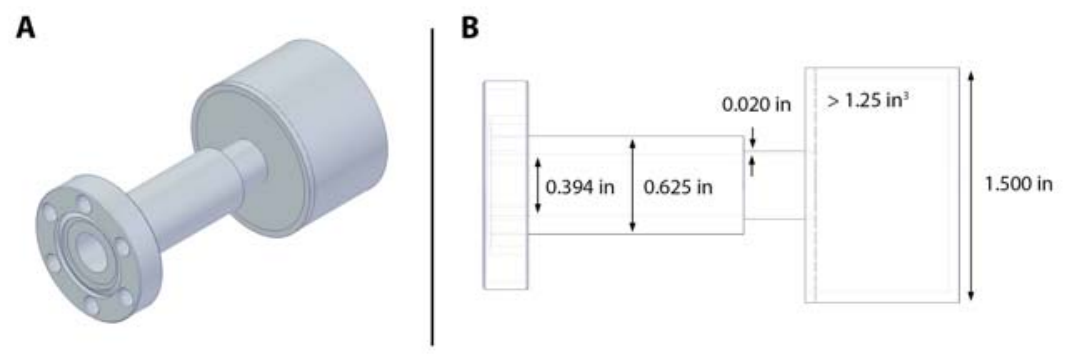

Figure 3.1: First generation source (G1). (A) The source consists of a stainless steel reservoir that is welded to a tube that in turn is welded to a 1-1/3 CF flange. A resistive band heater clamps around the reservoir. We wrapped the reservoir with a ceramic insulation and fit the reservoir between a pair of machined firebricks. (B) The tube connecting the reservoir to the flange has its diameter reduced to only 0.020 in at its connection to the reservoir in order to limit heat transfer away from the reservoir.

surface layer. We then cut the lithium under an argon atmosphere into pieces small enough to fit along the oven tube into the reservoir. We lined the oven tube with a 304 series stainless-steel cloth (250 openings per in and a 0.0016 in wire diameter) in an effort to minimize wasted material. Wire cloth has previously been used in recycling sources for enabling capillary action that transports wasted material back to a reservoir $[65]$.

A resistively heated band heater clamped around the reservoir cylinder and allowed for heating to beyond $600{ }^{\circ} \mathrm{C}$. We wedged a type-K thermocouple between the reservoir and the band heater for measuring temperature. We used a variac for applying power to the band heater. A crude temperature feedback scheme - implemented using a programmable microcontroller board ${ }^{2}$ - controlled a solid-state relay that regulated power to the variac. This setup maintained the reservoir temperature to within a few degrees Celsius by modulating the duty cycle for power applied to the variac in accordance with the deviation of the measured temperature from a desired setpoint. Software allowed for the setpoint to be varied controllably, enabling smooth control of the reservoir temperature.

We machined a pair of firebricks to clamp around the oven reservoir. We used

\footnotetext{
${ }^{2}$ Arduino Duemilanove. See: http://www.arduino.cc.
} 
a high-temperature cement to cover the firebricks with a nickel foil. Due to concern of molten lithium corroding copper gaskets, we used annealed nickel gaskets for mating the oven to the apparatus [66]. This source enabled some measurements when using enriched Li-6 at temperatures below $600{ }^{\circ} \mathrm{C}$. Due to its low capacity, the reservoir would empty quickly. More importantly, after prolonged use at our temperatures of interest $\left(550{ }^{\circ} \mathrm{C}\right.$ and beyond), lithium would clog the oven tube as a result of the temperature gradient between the reservoir and the tube.

Due to these drawbacks, we designed an alternative source (G2) - shown in Fig. 3.2 - for ensuing measurements using natural lithium at higher temperatures. This source included a substantially larger reservoir with an internal volume exceeding 4.75 $i^{3}$, allowing us to load the reservoir with tens of grams of lithium at a time. In contrast to the previous source, we heated the nozzle interfacing the reservoir to the apparatus to at least $50{ }^{\circ} \mathrm{C}$ beyond the reservoir temperature. We similarly loaded lithium through the nozzle into the reservoir, although we obtained natural lithium packaged under argon rather than mineral oil ${ }^{3}$. Wasted material accumulated in a large diameter tube without obstructing the relevant beam line. We lined this tube with a stainless steel foil for easily extracting wasted material when reloading the reservoir. The nozzle interfaced to this tube via a 0.040 in thick plate that choked off heat flow away from the nozzle. The entire assembly mated to the apparatus via a 2-3/4 in CF flange.

To heat this source we used highly flexible, small diameter heater cables. These cables consist of mineral (magnesium oxide) insulated heater wire enclosed in an Inconel 600 sheath. The heated ends of the cables terminate with splices to low resistance nickel wires (with 10:1 or higher resistance ratios) that in turn interface to lead wires that remain cool enough for handling and making connections to power ${ }^{4}$. A potted adapter hermetically seals the insulated cable assembly to prevent contamination by moisture. These cable assemblies can operate beyond $800{ }^{\circ} \mathrm{C}$ with large watt densities attainable.

We wound the heated ends of the assemblies into groove patterns that were

\footnotetext{
${ }^{3}$ We acquired lithium from ESPI Metals as 0.5 in diameter, 6.25 in long rods packed under argon. See http://www.espimetals.com.

${ }^{4}$ AeroRod heaters from ARi Industries, Inc. See http://www.ariindustries.com.
} 
A

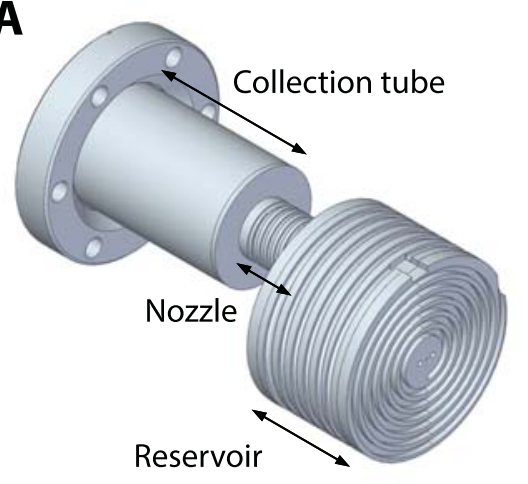

B

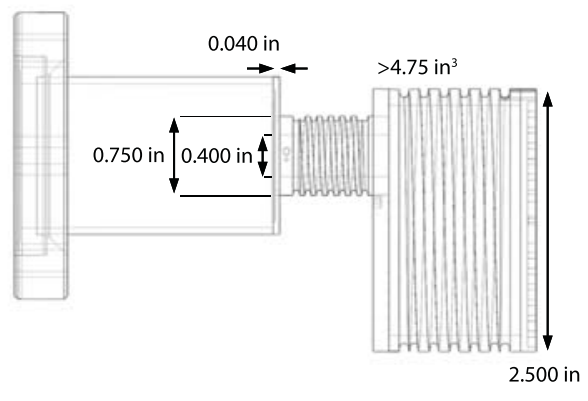

C

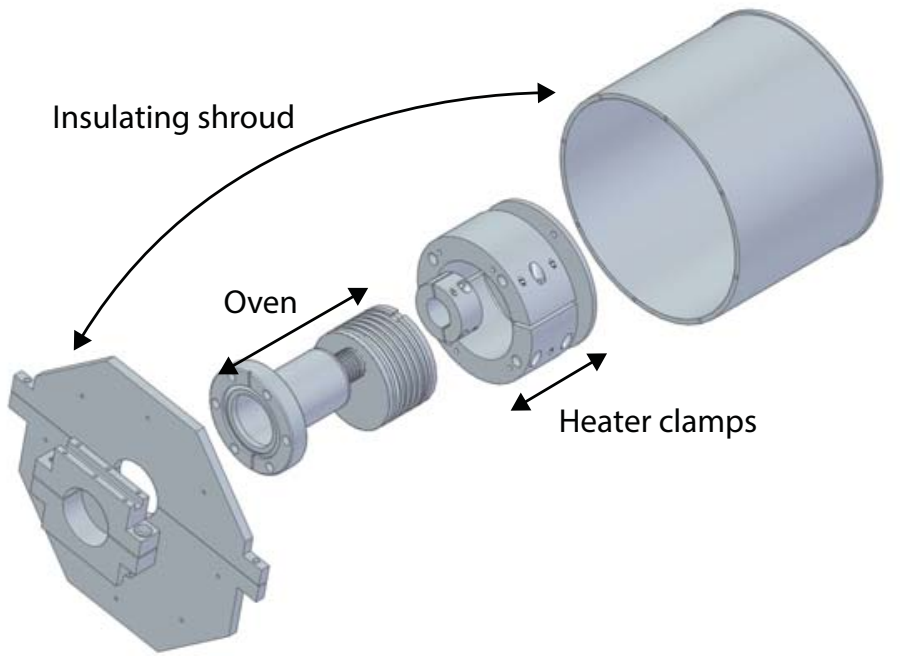

Figure 3.2: Second generation source (G2). (A) This entirely stainless steel source consists of a reservoir that is welded to a nozzle tube that in turn is welded to a commercial 2-3/4 in CF half-nipple. We wound heater cable into groove patterns on the nozzle and reservoir. In order to prevent clogging, we always heated the nozzle to $50{ }^{\circ} \mathrm{C}$ beyond the reservoir temperature. (B) The nozzle inner diameter was chosen to match the diameter of the exit tube on G1. The reservoir has a substantially larger capacity than G1, and by offsetting the nozzle from the reservoir axis we can fill the reservoir to beyond half its volume (without worrying about molten lithium spilling out of the nozzle). Material builds up on the walls of the half-nipple without obstructing the beam line. (C) Stainless steel clamps secure heaters around the nozzle and reservoir cylinders. A plate secures the heater on the rear of the reservoir in place by clamping into the reservoir clamp. A shroud builds off of the half-nipple and encloses the heated assembly. We lined the shroud with ceramic insulation. 
machined onto the reservoir and nozzle bodies. In particular, we used 38 in and 29 in (heated length) cables with 0.093 in diameter for the cylindrical body and rear plate of the reservoir, respectively. We used a 13 in (heated length) cable with 0.062 in diameter for the nozzle. The groove pattern depths exceed the cable radii in order to both enhance heat dissipation into the oven and allow for the heaters to be clamped in place. Stainless steel clamps fasten the heaters wound around the cylindrical bodies in place, while a stainless steel plate that bolts onto the larger clamp secures the rear heater in its groove pattern. We only used titanium bolts - lubricated using a boron nitride aerosol - with these clamps.

We press fit 0.032 in outer diameter, mineral insulated thermocouple assemblies into holes at several locations of interest on the oven ${ }^{5}$. These thermocouples measured temperatures T1, T2, and T3 at the following locations: the far end of the nozzle, center point of the rear plate, and interface between nozzle and reservoir. Similarly to G1, we implemented feedback-based temperature control using programmable microcontroller boards for modulating the duty cycle for applying power to the heaters ${ }^{6}$. In particular, a pair of boards employed software-based proportional-integral-derivative (PID) feedback to control relays wired in series with the nozzle and reservoir heaters. One variac powered the nozzle heater, while a second variac powered the reservoir heaters (which were wired in series). This setup allowed us to maintain temperatures $\mathrm{T} 1$ and $\mathrm{T} 2$ to within $1{ }^{\circ} \mathrm{C}$ of their steady-state setpoints.

A stainless steel shroud clamps around the collection tube and contains the heated assembly. Alkaline earth silicate insulation lines the volume between the oven and the shroud. We used nickel foil to further insulate the oven from the shroud exterior. Thermocouple and power connections feed out through holes on the rear plate of the shroud. Water cooling - including a heatsink on the cold end of the collection tube, copper tubing wound around the shroud cylinder, and a heatsink on the rear of the shroud - and silicone foam on the shroud keeps the exterior at safe temperatures.

\footnotetext{
${ }^{5}$ Super OMEGACLAD XL Heavy Duty Transition Junction Thermocouple Probes. See: http://www.omega.com.

${ }^{6}$ OsPID: The Open Source PID Controller. See http://www.ospid.com.
} 
While G2 circumvented issues that we encountered with G1, the heater on the nozzle failed multiple times at temperatures beyond $700{ }^{\circ} \mathrm{C}$. We attributed these failures to multiple issues relating to the nozzle heater including: small wire diameter, short length, and poor clamping. We suspect that these factors led to wear due to both rapid changes in heater temperature while regulating power and the presence of hot-spots resulting from uneven power dissipation along the heater length. We ultimately replaced the nozzle heater with a pair of heaters, with one providing latent heat at a constant power output and the other being regulated for fine-tuning the nozzle temperature. We replaced the stainless steel clamp with nickel foil that we fastened around the heaters using ceramic sleeving woven from alumina, boria, and silica fibers.

For our highest temperature measurements, we built a third-generation source (G3), shown in Fig. 3.3. The layout of this source is almost identical to G2. We increased both the diameter and the length of the nozzle in order to accommodate a longer heater with larger diameter (29 in heated length with 0.093 in outer diameter). We also increased the reservoir size to further augment its capacity. We modified the clamps for securing the heaters in place - notably making them out of copper - to more uniformly dissipate power along the heater lengths. We also potted thermocouples in place using a thermally-conductive metallic adhesive ${ }^{7}$. The only difference in the control scheme for this oven is that we operated the reservoir heaters using independent variacs (but a common control board) due to their substantially larger resistances. This oven mates to the apparatus using a $4-1 / 2$ in CF flange. With this oven, we were able to operate at temperatures up to $800{ }^{\circ} \mathrm{C}$ without heater failures.

For reference, G3 uses three 0.093 in diameter heater assemblies with heated lengths of 29 in (nozzle), 82 in (reservoir cylinder), and 53 in (rear plate). The measured resistances across these assemblies are 15.1, 42.6, and $27.5 \Omega$, respectively. At peak operating temperatures, we typically run the variacs for the respective heaters at roughly 60,70 , and $60 \mathrm{~V}$ with the feedback modulating the duty cycle at close to $50 \%$. We derive estimates for the energy efficiency of our source based on these figures-of-merit.

\footnotetext{
${ }^{7}$ Durabond 952 Nickel Based $2000{ }^{\circ} \mathrm{F}$ Adhesive. See: http://www.cotronics.com.
} 


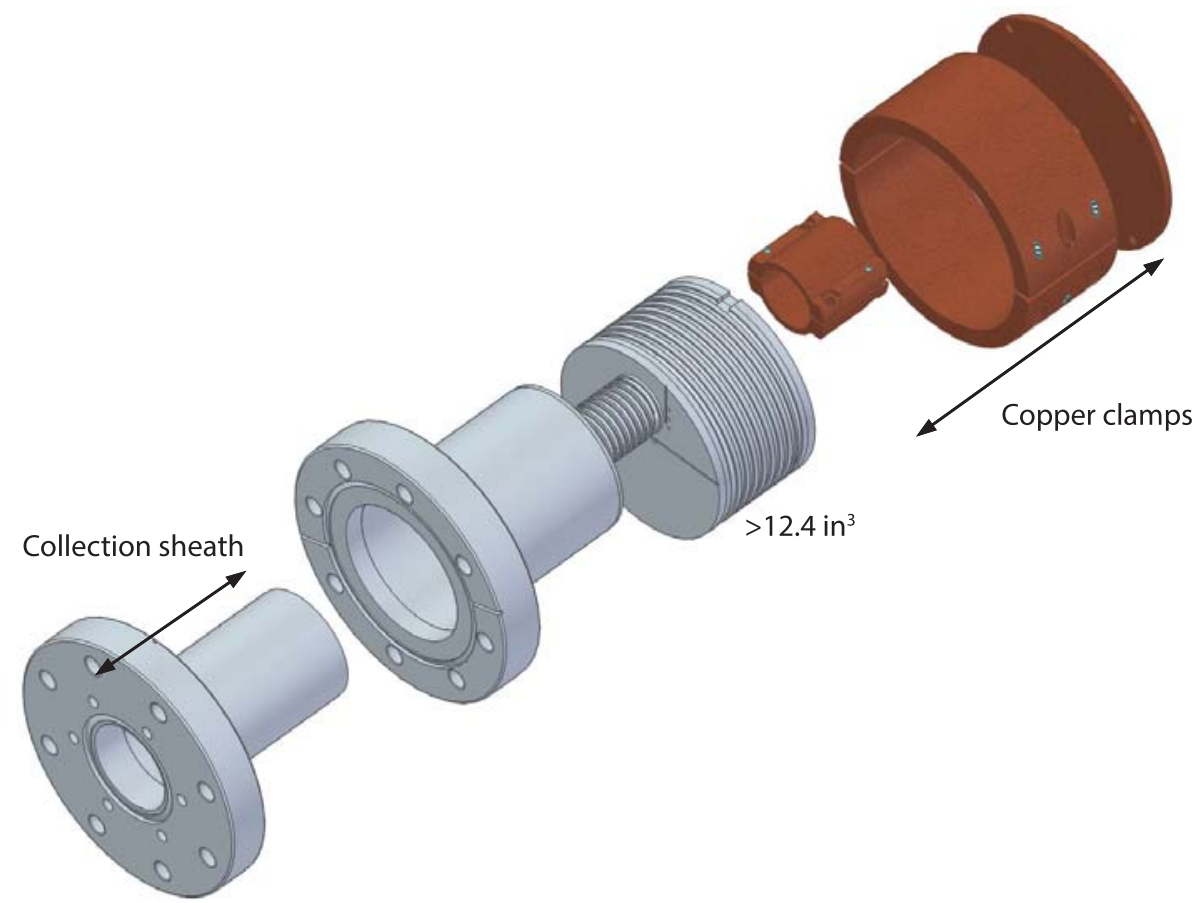

Figure 3.3: Third generation source (G3). This source is nearly identical to G2. The nozzle is slightly larger in order to accommodate a longer, larger diameter heater, and the reservoir has an even larger capacity. By using a 4-1/2 in CF half-nipple, we allowed enough space to fit a removeable collection sheath for easily extracting material that builds up during operation. We used copper clamps of a slightly different design than the previously used stainless steel clamps (in order to more uniformly spread heat across the assembly).

Immediately upon loading with lithium we mounted each source onto a separate apparatus with a viewport in front of the oven opening and a residual gas analyzer (RGA). For sources G2 and G3, we initially heated the assembly to just above 100 ${ }^{\circ} \mathrm{C}$ using constant power on the heaters. This procedure served to bake any water content out of the heater assemblies. We then heated a given source slowly to its peak operating temperature while monitoring contaminants (notably hydrogen) outgassing on the RGA. We simultaneously monitored the viewport in front of the oven opening. Via this degassing process, we baked a large fraction of contamination out of the reservoir while ensuring that a clean, uniform layer of lithium coated the viewport. 


\subsubsection{Source Aperture}

We positioned a $10 \mathrm{~mm}$ tall by $1 \mathrm{~mm}$ wide aperture at the output of the nozzle for every source. This aperture serves to prevent lithium from unnecessarily contaminating hardware in the apparatus. More importantly, however, the aperture provides a well-defined location for determining the initial conditions of atomic trajectories. The aperture width geometrically determines both a Doppler spread that must be addressed during optical pumping, and also a distribution for the incidence angles of atoms on the subsequent magnet array. We generated trajectories in our numerical simulations within the area defined by the aperture. As long as the extent of the source output is large enough, the source should in fact produce trajectories that populate the entire phase space determined by the aperture dimensions.

The vertical aspect ratio for the aperture is an important feature of the experimental setup. Choosing a narrow width (notably in comparison to the width of an aperture at the entrance of the magnet array), we can readily obtain a compromise between efficiency and purity by rotating the source about the guide entrance (as discussed in more detail later). Given the planar arrangement of the magnets, we can enhance flux simply by extending the height of the aperture at a given source temperature. Likewise, extending the source height can allow the source temperature to be relaxed which can both simplify source design and contribute to higher efficiency for the magnet guide. The source extents will need to scale with the aperture height in order to enhance flux.

Fig. 3.4 shows the setup for interfacing the aperture to source G1 (and G2 for a few measurements). We initially machined the aperture itself onto $1 / 16$ in thick stainless steel plate whose interior we thinned down to just 0.020 in. We bolted the aperture onto a custom 2-3/4 in CF gasket that included threaded holes for securing the stainless steel plate and a cutout in order to not impede any atomic trajectories. At the edge of the gasket we machined a notch that fit a key that we machined out of shim brass stock. When we set the gasket in place between the apparatus and a zero-length reducer interfacing to the source (G1), we ensured proper alignment of the aperture using this notch and key. 

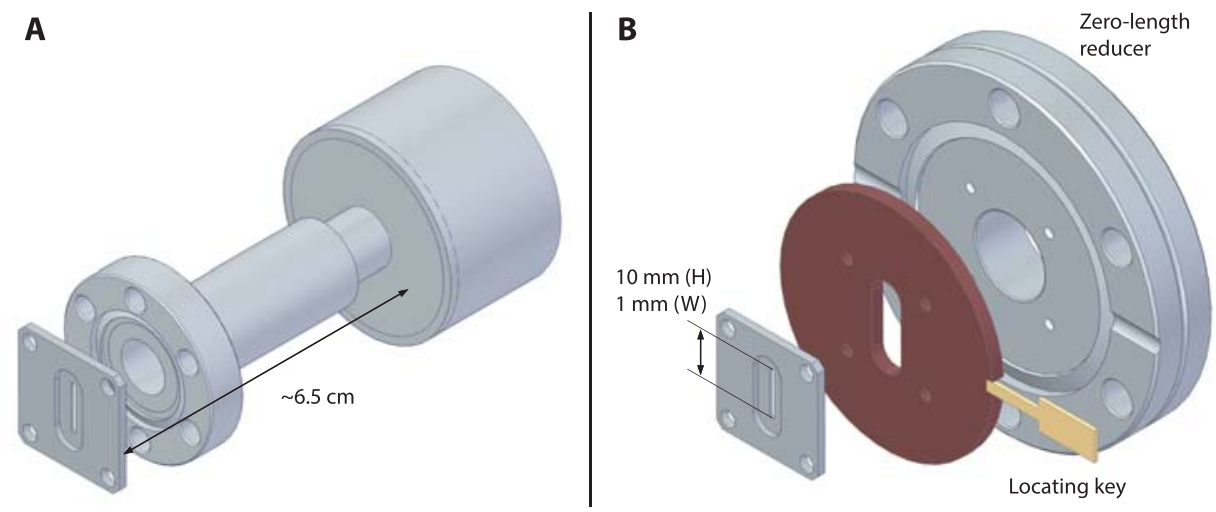

Figure 3.4: Source aperture used for lower temperature measurements (primarily with G1). (A) We machined the $1 \mathrm{~mm}$ wide by $10 \mathrm{~mm}$ tall aperture onto a 0.020 in thin section of a stainless steel plate that sat roughly $6.5 \mathrm{~cm}$ downstream from the exit of the reservoir. (A) The stainless plate bolted onto a machined $2-3 / 4$ in CF gasket. A notch on the edge of this gasket (together with a corresponding key machined out of shim stock) allowed us to ensure alignment of the aperture when securing the oven to the chamber via a zero-length reducer.

We grew concerned about using this aperture at elevated source temperatures for extended periods due to lithium building up around the aperture opening. Lithium build-up around the aperture could effectively increase the aperture thickness by enough to reduce its effective width by a measurable amount (as observed in the throughput beyond the magnets). If the source operated for long enough without cleaning off the aperture assembly, we feared that the aperture would become clogged. Due to the small capacity of source G1, not enough material condensed around the aperture to produce a noticeable effect on throughput between refilling the reservoir. Upon transitioning to source G2, we decided to modify the aperture assembly in order to prevent lithium condensation immediately around the opening.

Fig. 3.5 shows the layout for the aperture assembly that we used with sources G2 and G3. We heated the aperture itself to beyond the source operating temperature. The core of the assembly consists of a 1.225 in outer diameter, recessed stainless steel disk. In the recess we installed a serpentine tungsten filament sandwiched between a pair of 0.040 in thick ceramic disks. We machined the aperture onto a 0.030 in thick stainless steel disk that compresses the ceramic plates and filament into the recess of the opposite 
stainless steel disk. The ceramic disks were made Shapal-M: a highly machinable ceramic with unusually high thermal conductivity and very low outgassing rates even at high temperatures $^{8}$. The tungsten filament consisted of five turns of 0.040 in diameter wire that fit inside a 0.925 in diameter profile ${ }^{9}$. Fins machined onto the aperture disk lined cutouts on the ceramic disks (for the atomic beam) and the center-most gap of the filament in order to prevent corrosion (notably of the ceramics) by incident lithium.

This assembly was spring loaded onto shoulders machined on four titanium standoffs that were built off of a custom CF nipple ${ }^{10}$. These standoffs were thinned down to just 0.035 in along most of their lengths in order to choke off heat transfer to the nipple. A 0.032 in outer diameter, mineral insulated thermocouple fit through holes machined on the aperture and ceramic disks in order to press against the recessed disk. The thermocouple leads and the current leads to the tungsten filament fed out through of the nipple through a pair of arms terminating with 1-1/3 CF flanges. Applying 25 A (DC) through the filament (at close to $1 \mathrm{~V}$ ), the thermocouple reached temperatures beyond $700{ }^{\circ} \mathrm{C}$. At this current, the aperture disk glowed bright red, suggesting that the temperature at the aperture exceeded this temperature. We used the design for this heated aperture as the foundation for an assembly that rapidly heats a silicon wafer to beyond $900{ }^{\circ} \mathrm{C}$ for in-situ cleaning of the wafer surface [67].

\subsection{Laser System}

\subsubsection{Commercial System}

For most of our measurements, we used a commercial laser system that consists of a pair of grating-stabilized external-cavity diode lasers and a tapered amplifier ${ }^{11}$.

\footnotetext{
${ }^{8}$ Precision Ceramics manufactured the disks that we used for this assembly. See http://www.precision-ceramics.com for details on Shapal-M (among other interesting technical ceramics).

${ }^{9}$ The R.D. Mathis Company wound the filaments that we used in the assembly. See http://www.rdmathis.com.

${ }^{10}$ Retaining rings and tungsten springs from Kimball Physics were used for spring loading the assembly. See http://www.kimballphysics.com.

${ }^{11}$ We acquired this laser system from Toptica Photonics (DL pro, TA pro, and relevant electronics modules). See http://www.toptica.com.
} 

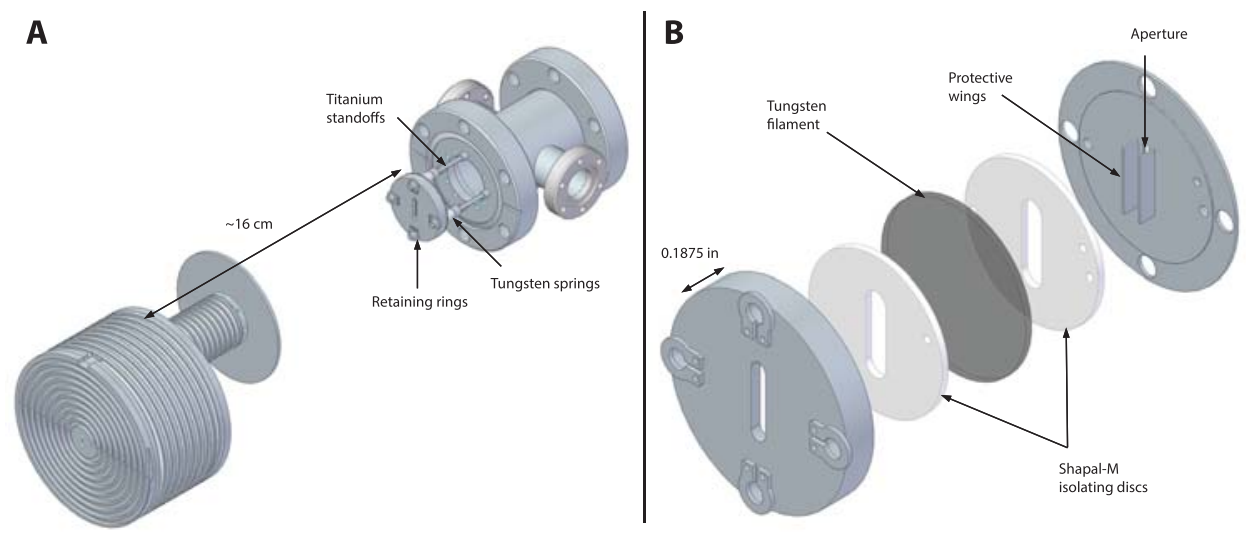

Figure 3.5: Heated source aperture used for higher temperature measurements (using G2 and G3). (A) The heated aperture sits roughly $16 \mathrm{~cm}$ from the reservoir opening for source G3. We built the aperture off of a custom CF nipple. (B) The aperture assembly consists of a pair of ceramic disks and a tungsten filament that are spring loaded into a recess in a stainless steel disk by a thin stainless steel disk (on which the aperture is machined). A thermocouple presses against the recessed disk. With roughly 25-30 W into the filament, we observe the assembly glowing bright red and the thermocouple measures the temperature to be greater than $700{ }^{\circ} \mathrm{C}$.

Fig. 3.6 outlines the optical setup for these commercial modules. We use one diode laser (spectroscopy laser) as a frequency reference, while the other diode laser (seed laser) seeds the tapered amplifier. As this system was acquired for previous work, more detail about the laser and optical setup of this system can be found in other dissertations $[47,48]$. Both diode lasers produce usable output powers of approximately $20 \mathrm{~mW}$, while the tapered amplifier provides between 300 and $400 \mathrm{~mW}$ directly at the output of the module.

We stabilize the frequency of the spectroscopy laser via frequency modulation spectroscopy using the Li-6 D lines as a reference [68]. The spectroscopy laser output passes through a pair of anamorphic prisms for reducing astigmatism in the beam shape. The laser then passes through an optical isolator that prevents light from subsequent reflections on optical surfaces from feeding back into the diode. A combination of waveplates and polarizing beamsplitter cubes divert fractions of the laser power to various parts of the setup with roughly even power. Some power double passes a lithium vapor cell for stabilizing the laser frequency. Other power generates beat frequencies on pho- 


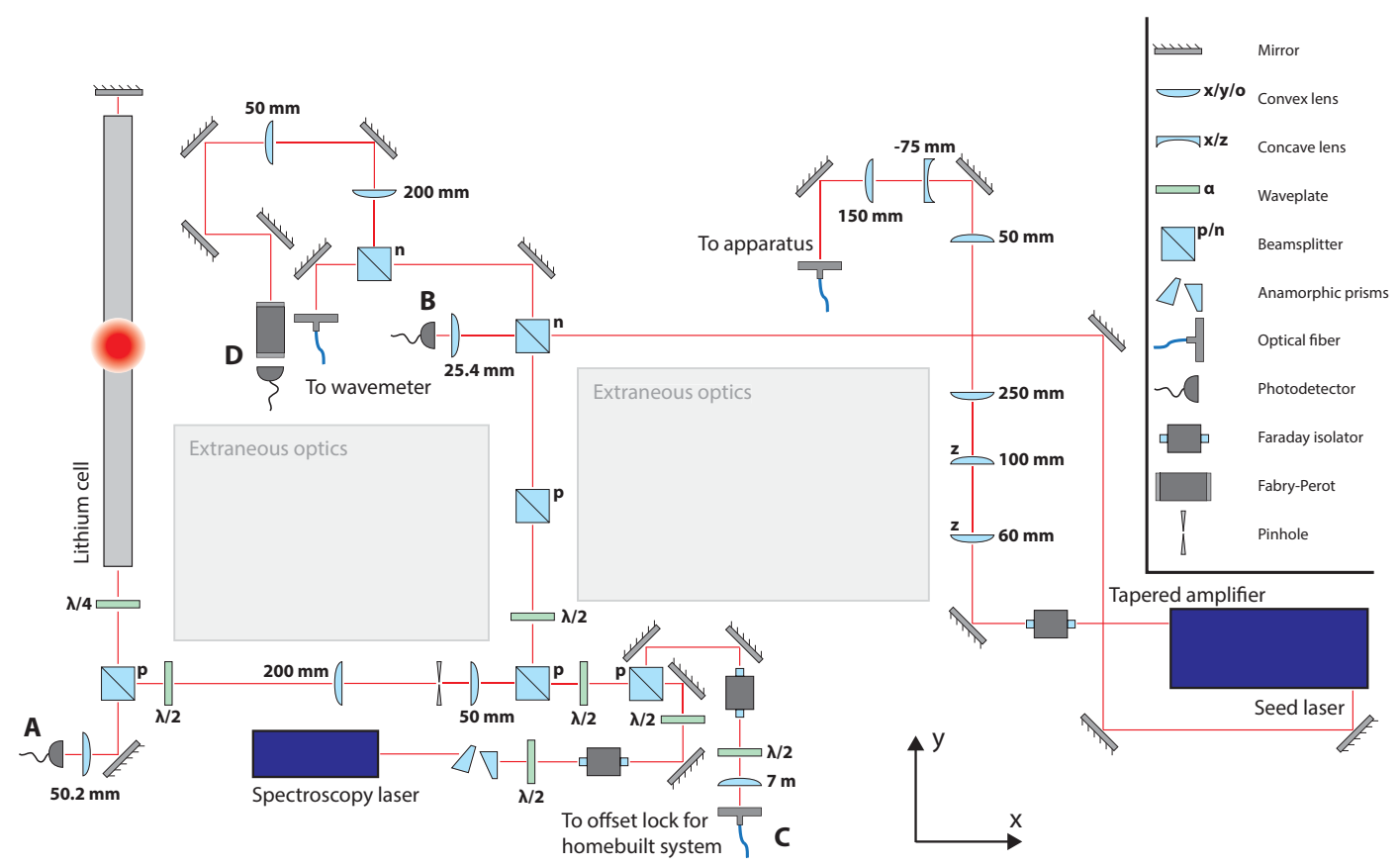

Figure 3.6: Laser system used for bulk of measurements. (A) The spectroscopy laser double-passes a lithium vapor cell in order to stabilize the frequency to the Li-6 D1 line. (B) We superimpose the spectroscopy and seed lasers on a photodiode in order to generate a beat note that we use for generating an error signal that allows us to determine the frequency offset between the lasers. (C) We mostly used the tapered amplifier output for optical pumping, but for some measurements we used a home-built tapered amplifier assembly. When using this assembly, we similarly superimposed the spectroscopy laser with the seed laser for our home-built setup. (D) We use a Fabry-Perot interferometer and wavemeter for diagnostics. 
todiodes upon mixing with small fractions of power that we sample from seed lasers for both the commercial tapered amplifier and a home-built tapered amplifier that we later implemented. The remaining power feeds a diagnostic setup including a Fabry-Perot interferometer and a commercial wavemeter ${ }^{12}$.

The lithium vapor cell consists of a long stainless steel tube with CF viewports on its ends and a sample of Li-6 (nominally enriched to 95\%) at its center. Heating the center of the cell to beyond $400{ }^{\circ} \mathrm{C}$ yields a sufficient vapor pressure of lithium for generating an absorption signal with the incident laser. Maintaining the cell at 35 mTorr (at room temperature) using argon prevents lithium atoms from reaching the viewports. Briefly, an electronics module for the spectroscopy laser adds frequency sidebands to the laser by modulating the diode current. While sweeping the central laser frequency (by ramping the voltage on a piezo that governs the grating position), a photodiode monitors the spectroscopy laser intensity after double-passing the cell. The resultant signal feeds into another electronics module that yields an error signal like that shown in Fig. 3.7(A). The zero-crossings correspond to spectral features of a Li-6 D line: two correspond to transitions from the hyperfine $\mathrm{F}=1 / 2$ and $\mathrm{F}=3 / 2$ ground states to the relevant excited state (depending on the $\mathrm{D}$ line), while the other (center-most) is a "cross-over" transition whose frequency is precisely halfway between the other two.

After stabilizing the spectroscopy laser, we stabilize the seed laser via a frequencyoffset lock by superimposing the spectroscopy and seed lasers on a photodiode [69]. By ramping the frequency of the seed laser (again by sweeping the voltage on a piezo that determines the laser cavity length), we vary the resultant beat frequency on the photodiode. We then mix the beat frequency with the output of a voltage-controlled oscillator using a frequency mixer. The output of the mixer is split and then recombined on a phase detector after one part passes a known delay length. The output of the detector produces an error signal - shown in Fig. 3.7(B) - whose zero-crossings correspond to detunings between the spectroscopy laser and the seed laser. By adjusting the frequency output of the oscillator, we can dynamically adjust the detuning. When operating the

\footnotetext{
${ }^{12}$ We use an EXFO wavemeter (WA-1000). See http://www.exfo.com.
} 

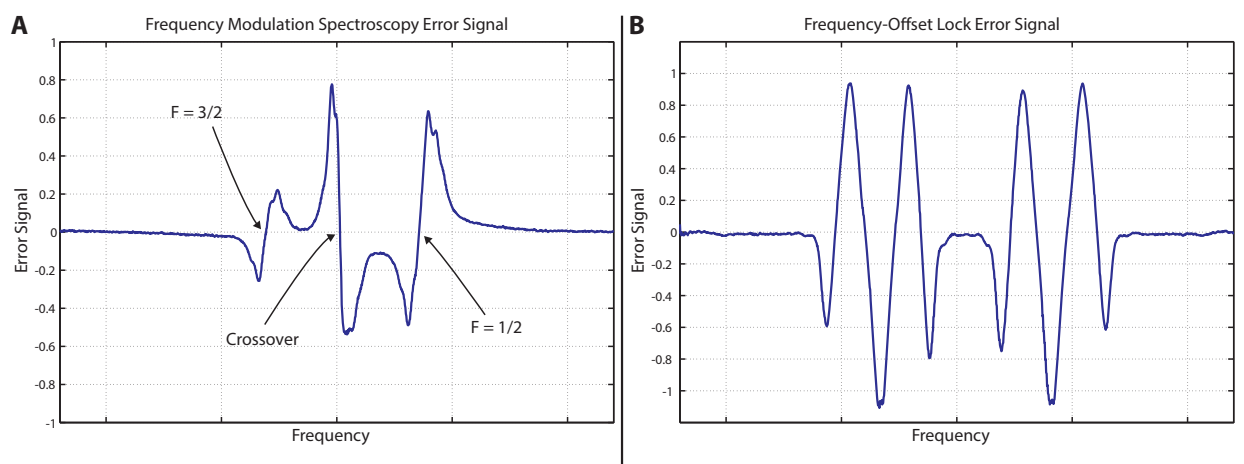

Figure 3.7: Error signals produced for stabilizing the spectroscopy and seed lasers. (A) The spectroscopy laser (with frequency sidebands produced by modulating the diode current) double-passes a lithium vapor cell. The resultant intensity (monitored while ramping the laser frequency) on a photodiode ultimately yields an error signal whose zero-crossings correspond to features of a particular Li-6 D line. (B) We generate a beat note on a photodiode by superimposing the spectroscopy (stabilized) and seed lasers. We mix the beat note with a known frequency, then split the output of the mixer. After imposing a phase delay between the resulting lines, we generate an error signal whose zero-crossings give particular detunings between the spectroscopy and seed lasers.

commercial system, we lock the spectroscopy laser to the "cross-over" transition of the Li-6 D1 line. We then lock the seed laser with a $114 \mathrm{MHz}$ offset (red-detuned) in order to have the tapered amplifier frequency be resonant with the D1 transition from the $\left|2^{2} \mathrm{~S}_{1 / 2}, F=3 / 2\right\rangle$ ground state.

The output of the tapered amplifier first passes an optical isolator to again mitigate feedback (and also to protect the amplifier). A combination of cylindrical and spherical telescopes then produce a more symmetric spatial profile for the output beam prior to coupling into a single-mode, polarization-maintaining optical fiber. This fiber runs roughly $15 \mathrm{~m}$ to the apparatus. Due to the poor mode quality of the tapered amplifier output, we typically couple between only 100 and $150 \mathrm{~mW}$ into the fiber. On a few occasions, we diverted the laser to the apparatus through free space in order preserve laser power. 


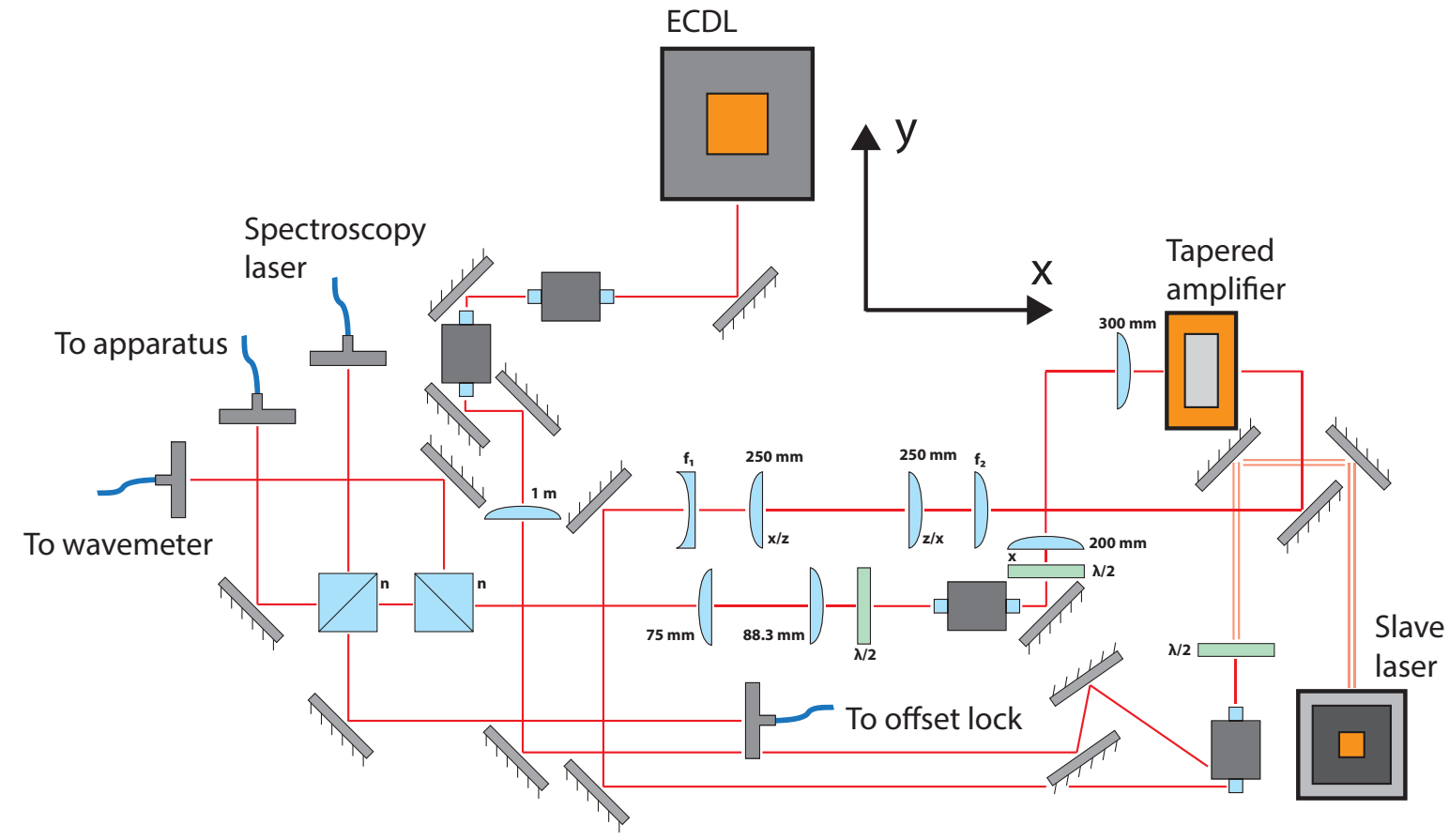

Figure 3.8: Home-built laser system. We recycled an assembly for a grating-stabilized, external cavity diode laser. We injected the output of this master laser $(<5 \mathrm{~mW}$ following isolators) into a slave laser diode that yielded more than $50 \mathrm{~mW}$ of usable power. The slave laser seeded a tapered amplifier that in turn produced up to $400 \mathrm{~mW}$ of usable power. We superimposed a fraction of light from the tapered amplifier onto the spectroscopy laser in order to establish a frequency-offset lock at the frequency difference between the D1 and D2 lines in Li-6.

\subsubsection{Home-built System}

During our earlier measurements, we shared the commercial laser system with colleagues who had setup this system for laser cooling Li-6 atoms on the D2 line. As we needed to operate on the D1 line and the tapered amplifier provided insufficient power for sharing, we quickly setup a home-built laser system in order to facilitate measurements. Using a small fraction of the output power of the spectroscopy laser (see Fig. 3.6(C)), we stabilized our laser to the D1 line using a frequency-offset lock at $\sim 10.05 \mathrm{GHz}[41]$. Fig. 3.8 schematically outlines our system.

For the front-end of our system, we recycled an external-cavity grating-stabilized diode laser that had previously been used for laser cooling cesium atoms [70]. This 
brass assembly, including a mount for the laser diode, an arm that flexes for the grating, and a baseplate for defining the external cavity length, mounts onto an aluminmum heatsink with a thermoelectric cooler sandwiched between the heatsink and baseplate. A temperature controller regulates the cooler in order to maintain the laser assembly at a constant temperature. An acrylic box with a window for the laser output (oriented at Brewster's angle) built off of the heatsink encloses the brass assembly. In order to adapt the assembly for use at $671 \mathrm{~nm}$, we needed to laterally offset the exit window due to the change in the operating grating angle. We also needed to remove material from the laser diode mount in order to prevent clipping of the output beam.

We used a laser diode rated to produce $120 \mathrm{~mW}$ (single transverse mode) at 660 $\mathrm{nm}$ at room temperature ${ }^{13}$. To center the gain profile at $671 \mathrm{~nm}$, we operated the laser diode at close to $70{ }^{\circ} \mathrm{C}$. For stable operation at this elevated temperature, we appended an additional 2.5 in thick, water-cooled aluminum heatsink beneath the entire assembly. We use a commercial current controller for driving the laser diode, and a home-built protection circuit prevents damage to the diode due to voltage spikes or reverse-biasing $[71]^{14}$.

Due to high diffraction efficiency for the grating that we recycled, most light fed back to the laser diode and only a small fraction of light (a few mW) exited the assembly. The output beam passes through a pair of optical isolators for mitigating feedback into the laser diode ${ }^{15}$. We use this master laser to stabilize the frequency of a slave laser diode via frequency pulling. The slave diode (same model as that used in the master laser, but not embedded in an external cavity) provides substantially higher power (beyond $50 \mathrm{~mW}$ ) for seeding a tapered amplifier. A pair of mirrors injects the master laser into the slave diode through an optical isolator. Operating the slave laser at close to threshold, we optimize the injection by maximizing the slave power while

\footnotetext{
${ }^{13}$ We bought a Hitachi (part no. HL6545MG) laser diode from ThorLabs. See http://www.thorlabs.com.

${ }^{14}$ We salvaged several old models of current and temperature controllers from both Newport Corporation and Wavelength Electronics. See http://www.newport.com and http://www.teamwavelength.com.

${ }^{15}$ We utilized several isolators designed for use with $780 \mathrm{~nm}$ light. By suitably rotating polarizers, we achieved isolation in accordance with specifications at the expense of transmission efficiency.
} 

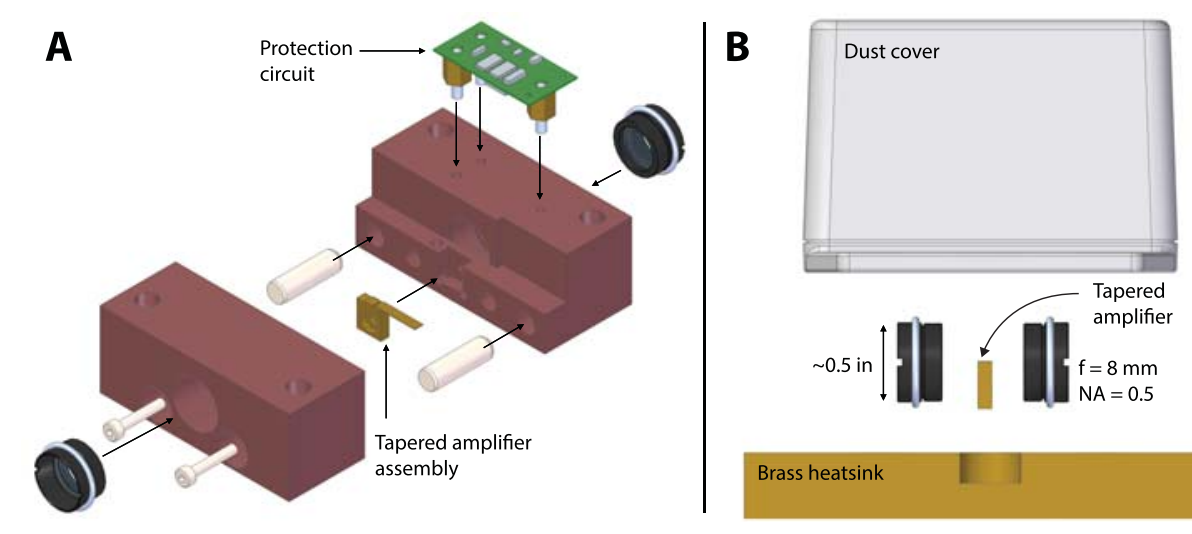

Brass heatsink

Figure 3.9: Tapered amplifier assembly. (A) The assembly consists of a pair of brass blocks. The tapered amplifier bolts into a recess on one of the blocks. The blocks then press together (along dowel pins that ensure proper alignment). Holes in the blocks define an optical axis, with the tapered amplifier itself sitting directly on axis at the center of the complete assembly. (B) A pair of aspheric lenses thread into the brass heatsinks in order to focus the seed laser onto the amplifier and then collimate the amplifier output.

tuning these folding mirrors. The slave assembly likewise consists of a brass mounting block that bolts onto an aluminum heatsink (with a thermoelectric cooler in place for regulating the slave diode temperature). A similarly massive, water-cooled aluminum heatsink sits beneath the assembly for facilitating operation at $70{ }^{\circ} \mathrm{C}$.

We use the slave laser output for seeding a tapered amplifier ${ }^{16}$. Fig. 3.9 shows the assembly that we built for seeding the tapered amplifier ${ }^{17}$. The amplifier itself bolts onto a recess in a brass heatsink using a custom copper shoulder washer. This heatsink press fits onto another heatsink, with holes through the heatsinks defining an axis for the incident beam. The tapered amplifier sits on this axis, and a pair of aspheric lenses thread into the holes on the heatsinks for focusing the incident seed beam onto the amplifier and collimating the resultant amplifier output. A protection circuit mounts on one of the heatsinks, and a dust cover encloses the assembly.

We use a pair of telescopes prior to the amplifier for shaping the incident beam

\footnotetext{
${ }^{16}$ We acquired a tapered amplifer from eagleyard Photonics (part no. EYP-TPA-0670-00500-2003CMT02-0000), which has since been acquired by Toptica Photonics.

${ }^{17}$ We acquired this assembly from the group of Professor Kirk Madison at the University of British Columbia.
} 
to match the profile of amplified spontaneous emission generated by the amplifier. An optical isolator directly follows the amplifier for preventing damage to the amplifier by feedback. After the isolator, we typically measure the laser power to be between 350 and $400 \mathrm{~mW}$. We divert a fraction of this power to both a wavemeter and Fabry-Perot interferometer. We superimpose another fraction of power onto the spectroscopy laser into an optical fiber. We measure the $>10 \mathrm{GHz}$ beat frequency on a photodetector with bandwidth in excess of $10 \mathrm{GHz}^{18}$. We use the resultant beat signal for frequency-offset locking the master laser to the D1 line (particularly from the $\left|2^{2} \mathrm{~S}_{1 / 2}, F=3 / 2\right\rangle$ ground state). Setting up the frequency-offset lock required careful choice of components due to the high bandwidth needed prior to mixing the beat frequency with a reference frequency ${ }^{19}$. We achieved feedback on the resultant error signal using home-built laser servo electronics that were used for previous experiments. We ultimately coupled up to $150 \mathrm{~mW}$ of the remaining power from the tapered amplifier into a single-mode, polarization-maintaining fiber that ran to the apparatus.

\subsection{Beam Line and Optical Pumping}

Fig. 3.10 shows the beam line (incorporating source G3) leading up to the magnet array. A 4-1/2 in CF bellows interfaces the beam line to the chamber housing the magnets, allowing the beam line to pivot about the magnet array. The source interfaces to the assembly for the heated $1 \mathrm{~mm}(\mathrm{~W})$ x $10 \mathrm{~mm}(\mathrm{H})$ aperture via a zero-length $\mathrm{CF}$ reducer. This assembly then mates to a 6-way 2-3/4 in CF cross. A rotary feedthrough connected to the top of this cross actuates the rotation of a stainless steel plate that mates to the feedthrough with custom standoff that offsets the plate from the rotation axis. This stainless steel "flag" functions as a beam block for the atomic beam. We orient

\footnotetext{
${ }^{18}$ We also acquired the photodetector from Professor Kirk Madison. The photodiode itself is from Advanced Optical Components (now Finisar). Its part number is HFD6X80-13 (no longer in production).

${ }^{19}$ Many components, particularly the frequency mixer and all subsequent components, were acquired from Mini-Circuits. See http://www.minicircuits.com. We acquired a sample for a VCO with output centered at $10 \mathrm{GHz}$ from Hittite Microwave Corporation (part no. HMC530LP5). See http://www.hittite.com. This VCO included a divide-by-four output that we used for monitoring the VCO frequency on a counter. We acquired high gain amplifiers that operate at bandwidths up to 10 GHz from RF Bay, Inc (notably part no. LPA-10-20). See http://www.rfbayinc.com.
} 

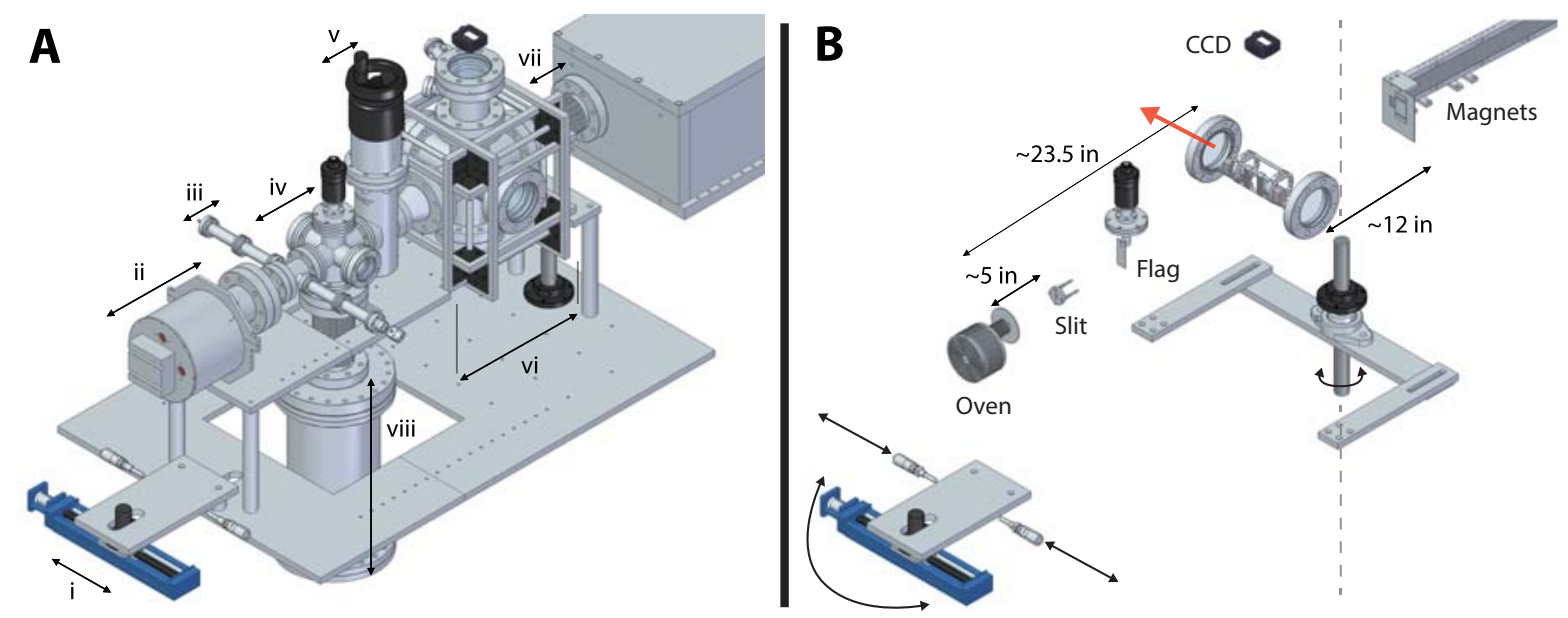

Figure 3.10: Beam line for optical pumping. (A) A linear actuator (i) determines the rotation of the entire beam line (including optics). The beam line consists of the source (ii), heated aperture (iii), 6-way 2-3/4 in CF cross with a rotatable flag for obstructing the atomic beam (iv), gate valve (v) for always maintaining the source under vacuum, and optical pumping region (vi). The pumping region includes a 6 -way $4-1 / 2$ in $\mathrm{CF}$ cross and three nested Helmholtz pairs for defining a quantization axis. A bellows (vii) interfaces the beam line to the chamber housing the magnets. Another bellows connects the beam line to a nipple (viii) that in turn connects to a large ion pump. (B) Key components of the beam line and relevant distances. The red arrow indicates the direction of the optical pumping beam. A radial bearing allows rotation of a shaft that is fastened to the lower support structure for the beam line via a flanged shaft mount.

the flag accordingly when not making measurements or making a relevant background measurement.

A custom edge-welded bellows with $4-1 / 2$ in CF flanges connects the cross to an 8 in $\mathrm{CF}$ nipple that in turn connects to a $270 \mathrm{~L} / \mathrm{s}$ ion pump ${ }^{20}$. This bellows was designed to withstand the substantial lateral offset between its flanges upon pivoting the beam line. Viewports on either side of the cross allow for inspection of the flag. Lastly, a gate valve connects to the end of the cross opposite the source ${ }^{21}$. Using this valve, we can maintain the source under vacuum when performing maintenance on subsequent sections of the apparatus. A custom gasket with an aperture of diameter close to 0.75

\footnotetext{
${ }^{20}$ We use a $270 \mathrm{~L} / \mathrm{s}$ Varian Galaxy Diode. Duniway Stockroom offers rebuilding services see http://www.duniway.com. We acquired the bellows from Standard Bellows Company - see http://www.std-bellows.com.

${ }^{21}$ This all-metal gate valve is from VAT Valve (part no. 48132-CE01). See http://www.vatvalve.com.
} 
in connects the valve to the cross. This aperture permits relevant trajectories into the optical pumping region while shielding the valve assembly from lithium contamination.

A conical reducer mates the gate valve to a 6 -way $4-1 / 2$ in CF cross where we perform optical pumping. The opposite side of the cross connects to the bellows that interfaces the beam line to the chamber containing the magnet array. Viewports terminate all other arms of the cross, with the viewports along the optical pumping arm having an anti-reflection coating at $671 \mathrm{~nm}$. The viewport on top of the cross is offset by a custom nipple that includes auxiliary ports for making electrical connections to a piezo-actuated variable aperture that restricts the transverse extent of the atomic beam in the pumping area. The viewport on the bottom of the cross mates to the cross through an aluminum support structure that supports the weight of the cross. We image fluorescence produced during optical pumping on a CCD camera (with a $25 \mathrm{~mm}$ lens) positioned above the cross ${ }^{22}$.

Three pairs of Helmholtz pairs fit around the cross for defining a quantization axis for optical pumping. We wound the coils around square Delrin frames with each coil including 44 turns (four layers of eleven turns) of insulated 22 AWG wire. The exact dimensions of the coils vary for each pair (in order to allow nesting of the coils), but we maintain the Helmholtz condition for every pair. That is, $\mathrm{D}=0.54 \mathrm{~L}$ for every pair, where D and L denote the distance between coils and edge length, respectively [72]. Delrin rods bolt to corner pieces on the Delrin frames for ensuring the proper spacing between coils. All three pairs bolt accordingly to the support structure for the cross in order to ensure that the pumping volume coincides with the center of the three pairs. We confirmed using a gaussmeter that we can generate 5 Gauss at the center of the pumping volume with each pair independently (using less than $3 \mathrm{~A}$, which is the peak current that we ever used due to heating). We used home-built current controllers for maintaining constant current through respective coil pairs [73].

The aluminum support structure that supports the pumping cross consists of a pair of plates with stiffening aluminum interconnects joining the plates. Cutouts on the

\footnotetext{
${ }^{22}$ We use a Point Grey Chameleon CMLN-13S2M camera with a Fujinon HF25HA-1B lens. See http://www.ptgrey.com.
} 
plates accommodate the bellows that connects to the ion pump with enough space to allow for a substantial lateral offset of the bellows flanges. Optical posts (1 in diameter) connect this upper support structure to a lower structure that likewise consists of a pair of aluminum plates that interconnect via stiffening plates (stainless steel in this case). The upper structure rests on flanged nuts that translate along threaded rod that extends from the optical posts. By adjusting these nuts, we can align the beam line to the following chamber housing the magnets.

The lower plates similarly include cutouts for the 8 in $\mathrm{CF}$ nipple that feeds to the ion pump. The lower structure rests atop a collection of ball transfers that screw into an optical table. An aluminum frame on the optical table positions a flanged radial bearing directly beneath the center of the bellows connecting the pumping cross to the magnet chamber. A 1 in stainless steel shaft pressed into this bearing feeds through a hole in the lower suport structure. The shaft clamps onto a flanged shaft mount that is bolted onto the lower support structure. This arrangement enables the entire beam line, including all vacuum hardware and optics mounted to the support structures, to rotate about the bellows connecting to the chamber containing the magnets.

A plate extends from the lower support structure away from the source. A cylindrical extension to a nylon puck fits through a slot machined at the end of this plate. The base of the puck mounts directly to a long-travel linear actuator that is bolted to the optical table perpendicularly to the beam line ${ }^{23}$. Moving the puck along the actuator (by turning a handwheel) enables rotation of the beam line until the extension on the puck reaches the end of the slot machined on the plate extending from the support structure. We bolted a pair of micrometers to the optical table for measuring the displacement of the plate (and thus the rotation of the beam line).

\subsubsection{Optical Setup}

We mounted breadboards on the support structure for setting up the optics for optical pumping. Fig. 3.11 shows the optics arrangment for preparing the optical pumping

\footnotetext{
${ }^{23}$ We used a linear actuator from Thomson Linear Motion (part no. MS33LGBL400). See http://www.thomsonlinear.com.
} 

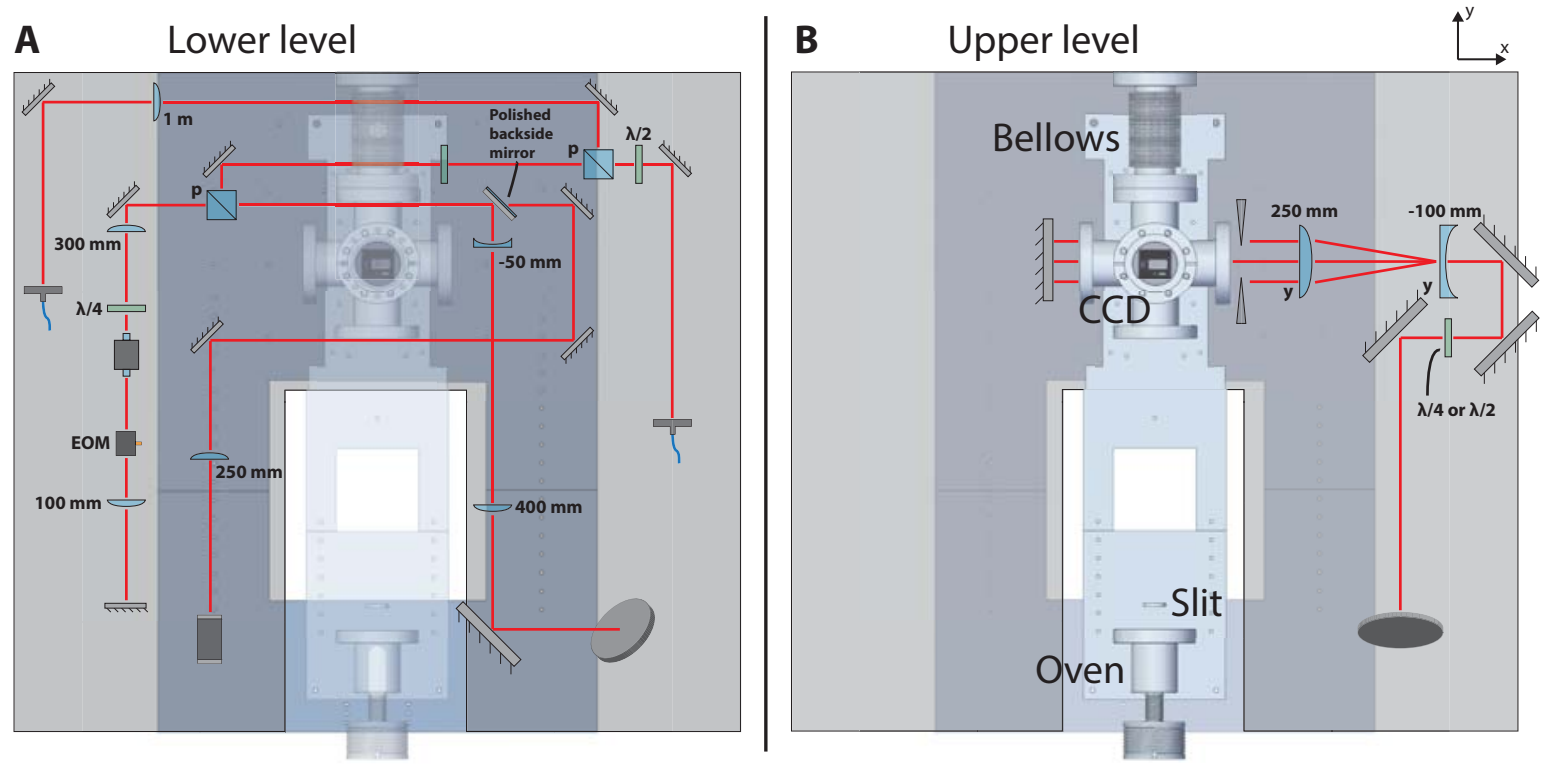

Figure 3.11: Laser beam path for optical pumping. (A) A Glan-Thompson polarizer at the output of the optical fiber from the laser setup reduces power fluctuations following polarizing optics. We divert some light to another fiber that runs to our setup for fluorescence detection. The remaining light double-passes an EOM that spectrally broadens the beam by adding frequency sidebands spaced by close to the natural linewidth of the Li-6 D1 line. The resulting beam is expanded using spherical and cylindrical telescopes prior to entry into the pumping chamber. (B) Just prior to entering the chamber, we use a waveplate for tuning the beam polarization. We also use a variable slit for adjusting the beam width (for investigating dependence on interaction time between atoms and the laser).

beam. A Glan-Thomposn polarizer first selects light from the laser system (coupled into a polarization-maintaining optical fiber) with the correct polarization. This polarizer suppresses power fluctuations beyond polarization selective optics (notably polarizing beamsplitter cubes). We then divert some light using a waveplate and polarizing beam splitter cube to another fiber that runs beyond the magnet array for detecting fluorescence. The remaining beam passes through a Faraday rotator in order to rotate the laser polarization by $45^{\circ}$. Double-passing the rotator directly rotates an incident linear polarization by $90^{\circ}$, in contrast to using a quarter-wave plate. A half-wave plate in front of the rotator determines the laser polarization incident on a subsequent electro-optic modulator (EOM), and a telescope surrounding the EOM focuses the beam onto the center of the EOM. 
Applying RF voltage to the EOM changes the extraordinary refractive index of a lithium niobate crystal that in turn induces a pure phase shift (with no change in polarization state) on an incident beam assuming that its polarization is linear and aligned with the extraordinary axis of the crystal. The resulting phase modulation results in frequency modulation of the input beam. In particular, the EOM produces frequency sidebands about the central frequency (separated by integral multiples of the modulation frequency) with the relative energy in the sidebands depending on the incident RF power. Our EOM introduces sidebands spaced by $6 \mathrm{MHz}$ (roughly matching the natural linewidth of the Li-6 D1 line) about the central frequency, with the half-wave voltage (corresponding to the peak-to-peak voltage that yields a $\pi$ phase shift) given by roughly $15 \mathrm{~V}$ ( or $2.25 \mathrm{~W}$ ). We use the EOM for tailoring the spectral width of the optical pumping beam to match the relevant Doppler profile for the atomic beam in the pumping region [74]. By double-passing the EOM, we introduce sidebands upon sidebands for further broadening. Fig. 3.12 shows the laser spectrum on a Fabry-Perot interferometer after double-passing the EOM while varying the incident $\mathrm{RF}$ power between 0 and $3 \mathrm{~W}$. We ideally would broaden the laser spectrum by introducing noise as modulation onto the current driving the laser diode that seeds the tapered amplifier [75]. We did not have the capability of implementing this modulation over the course of this work.

A small fraction of the light that double-passes the EOM passes through a polished-backside mirror for analysis of its spectrum on a Fabry-Perot interferometer. The remaining light (typically about $50 \mathrm{~mW}$ ) is expanded to about 0.5 in diameter using a cylindrical telescope and then diverted to the $4-1 / 2$ in CF cross for optical pumping. A waveplate and polarizing beamsplitter cube prior to the cross determine the final polarization. A cylindrical telescope finally expands the beam to a width of roughly 1.5 in for longer interaction time with the atomic beam. A variable slit that we jury-rigged from an adjustable spanner wrench tool determines the actual beam width incident on the atoms ${ }^{24}$.

\footnotetext{
${ }^{24}$ The tool came from ThorLabs (part no. SPW801). We mounted slit edges onto the wrench blades and interfaced the assembly to an optical post.
} 

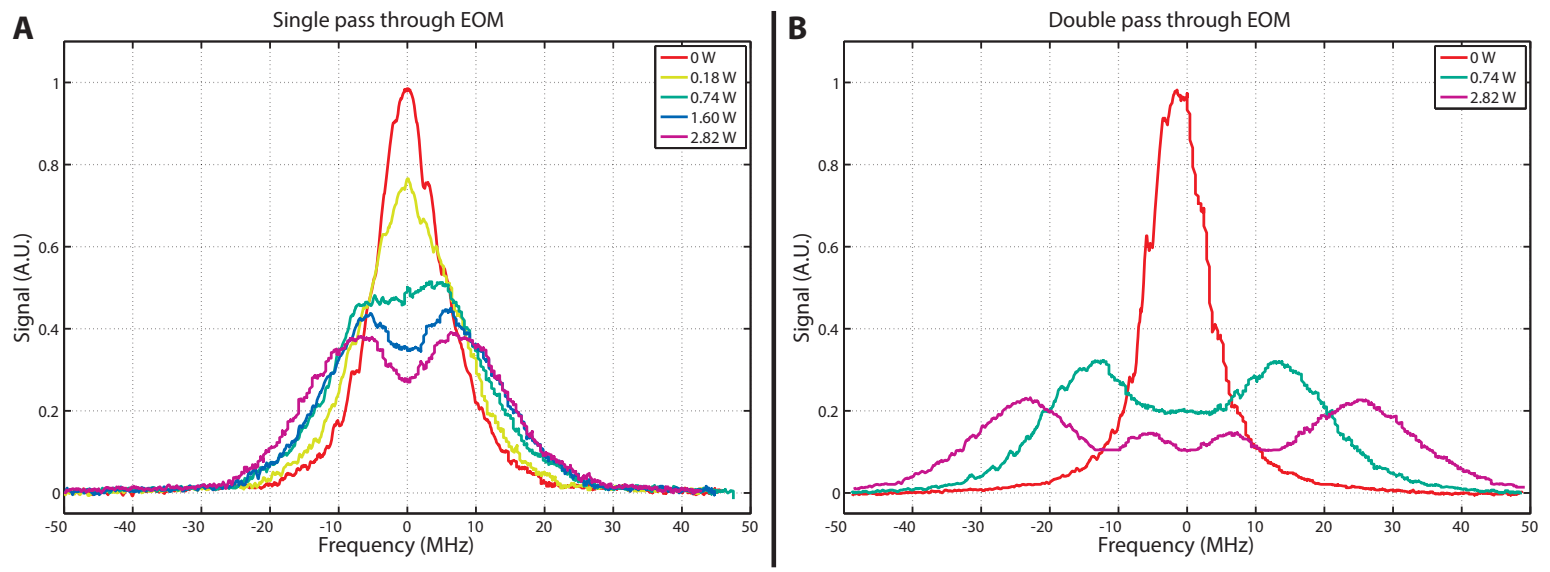

Figure 3.12: Spectrum broadening using an EOM. (A) With between 0 and $3 \mathrm{~W}$ incident on the EOM, a Fabry-Perot interferometer indicates that we can broaden the laser beam by more than $25 \mathrm{MHz}$ after a single pass. The power per unit frequency is clearly not uniform, and is likely worse than the measurements indicate due to the limited resolution $(7.5 \mathrm{MHz})$ of the Fabry-Perot. (B) Double-passing the EOM, we extend the spectral width to beyond $50 \mathrm{MHz}$.

\subsubsection{Variable Beam Slit}

For most measurements we included an aperture in the optical pumping chamber for restricting the atomic flux to just those trajectories that will be incident on an aperture at the entry to the magnet array. For all measurements, a custom gasket with a 0.950 in diameter aperture interfaced the pumping cross to the front-end of the beam line. As shown in Fig. 3.14, we initially built four rods off of this gasket that extended to the center of the cross. Beveled ends on these rods allowed us to slide a custom aperture in place without removing the gasket by reaching down from the top of the cross using a pair of tongs. We machined a set of gaskets with various apertures (all with rectangular aspect ratios). We initially aligned the orientation of the gasket and rods using the CCD above the cross.

With this system proving to be cumbersome due to the need for breaking vacuum in order to swap apertures, we designed a variable aperture using piezo-electric actuators as shown in Fig. 3.14. The frame for this assembly consists of four $4 \mathrm{~mm}$ rods secured (via set screws) to a pair of disks whose diameters closely fit the inner diameter of the pumping arm of the cross. These disks include windows that are sufficiently large for 


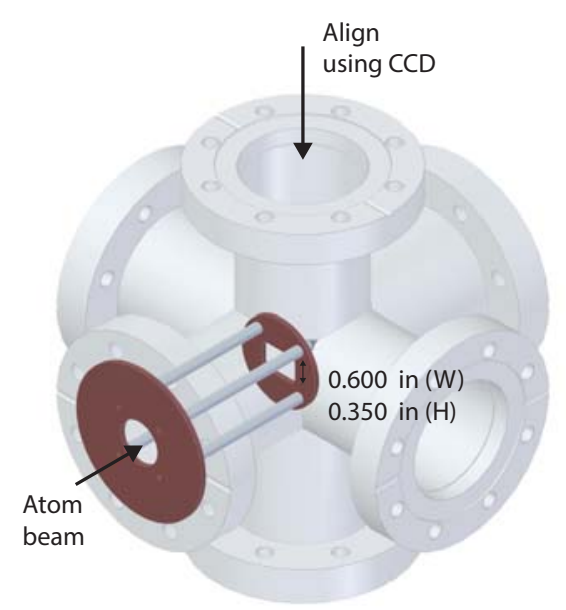

Figure 3.13: First aperture system used in the optical pumping chamber. Rods with beveled ends extended from a custom gasket (with a 0.950 in diameter) into the center of the pumping cross. Using tongs, we slid a second custom gasket including the desired aperture dimensions onto the rods. This setup allowed us to change apertures without disturbing the vacuum seal along the beam line. Swapping apertures along the rods, however, proved to be difficult.

passage of the optical pumping beam. Ultra-high vacuum compatible actuators that drive the motion of a pair of slit edges along two of the frame rods also mount onto these disks ${ }^{25}$. Springs between the slit edges and the disks on the frame allowed for bi-directional motion of the edges. With each actuator allowing for travel of 1 in, we could adjust the spacing between the edges to be between 0 and 1 in while displacing the midpoint between the edges by 0.5 in to either side of the center of the cross. While this assembly provided some insight into which trajectories contribute to which aspects of the distribution beyond the magnets, we encountered difficulty in achieving smooth travel of the slit edges along the frame and ultimately removed the assembly from the apparatus.

\footnotetext{
${ }^{25}$ We used piezo-electric actuators and an open-loop controller from Newport Corporation (part nos. 8302-UHV-KAP and 8742). See http://www.newport.com.
} 

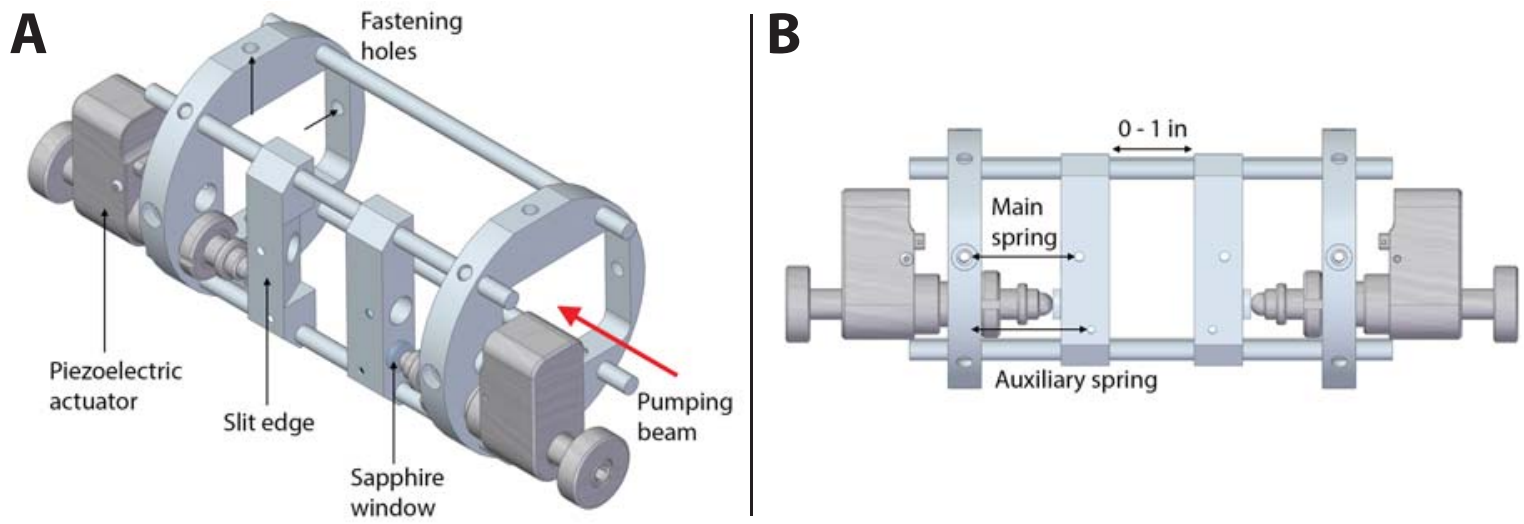

Figure 3.14: Variable slit used in the optical pumping chamber. (A) Piezo-electric actuators drive the motion of a pair of slit edges along two rods that comprise part of the frame for the entire assembly. Two disks that locate the rods closely fit the inner diameter of the pumping arm of the cross. Set screws screw outward from these disks in order to secure the assembly in place within the cross. (B) With the slit edges occasionally stalling on the frame rods (due to the actuator being offset from the midpoint between the rods), we added auxiliary springs and coated the rods with an ultra-high vacuum compatible lubricant ${ }^{a}$.

${ }^{a}$ We used Apiezon L as the lubricant. See http://www.apiezon.com.

\subsection{Magnetic Guide}

\subsubsection{Design and Construction}

We experimented with several designs for constructing the curved Halbach array. We ultimately agreed to approximate the analytical form of the array by fifteen panels of Halbach arrays with 0.5 in periodicity (i.e. using 0.125 in wide by 0.125 in thick permanent magnets with magnetization oriented through the thickness). We initially had planned on stacking panels for achieving enrichment over a height close to the height of the chamber housing the array. Prototypes indicated that stacking these panels (while minimizing the gap between magnets in adjacent layers) would be challenging. As throughput should scale linearly with the array height (given the planar arrangement), we ultimately reneged on the plan to stack panels and built fifteen panels using 0.125 in wide by 0.125 in thick by 1.5 in tall neodymium-iron-boron (NdFeB) magnets ${ }^{26}$.

\footnotetext{
${ }^{26}$ We acquired $\quad$ N52 grade NdFeB magnets from SuperMagnetMan. See http://www.supermagnetman.net.
} 

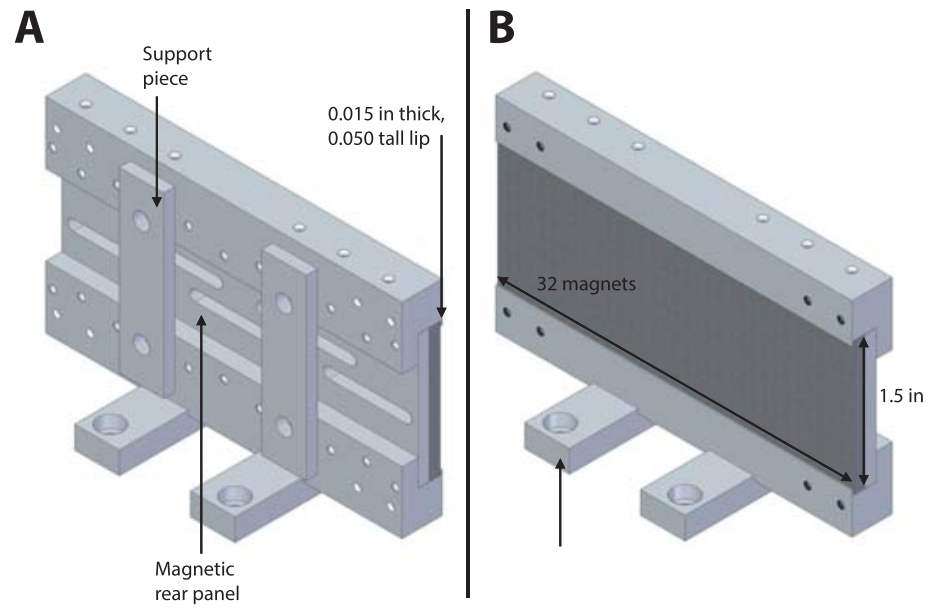

Figure 3.15: Magnet panel assembly. (A) Key components include a pair of support blocks with pockets for containing the magnets. One side of these pockets is just 0.015 in thick and 0.050 in tall. A 0.125 in thick plate whose lateral dimensions match the overall dimensions of the magnets sits in this pocket and presses against the magnets (via set screws through the opposite side of the pockets on the support blocks). A pair of framing plates fix the spacing between the support blocks. (B) Magnets slide into the pockets on the support blocks along the 0.125 in thick plate. Cutouts on the plates that press against the magnets ensure no virtual outgassing.

Fig. 3.15 outlines the design for one of the panels that we used for making the array. Most components for the panels were machined from 410 series stainless steel, a mildly magnetic grade with low outgassing characteristics. The ends of the magnets fit into pockets machined along the 4 in lengths of a pair of support blocks. One side of these pockets is just 0.015 in thick and 0.050 in tall in order to minimally obstruct the surface of the Halbach array while still providing strong enough support for keeping the magnets flat. A pair of framing plates bolt to the thicker and deeper side of the pockets in order to configure the spacing between the blocks to match the magnet height. A 0.125 in plate sits against this side of the pockets in the blocks. Set screws (with flat tips) through the blocks into the pockets press this plate against the magnets which in turn become secured in place between the plate and the lip on the opposite side of the blocks. We tightened the set screws and the framing plates accordingly during assembly for easily allowing magnets to slide into the pockets from the end of the panel while 
simultaneously fastening installed magnets in place ${ }^{27}$. A pair of feet bolted to the base of the resulting assembly allow us to interface the array to the chamber.

Fig. 3.16 shows the mechanism for linking panels together in the assembly. Stainless steel shim stock (0.010 in thick) secures panels next to each other (on the face of the panels) while still allowing the panels to bend about their shared edge. After appending a panel to the array using these thin interconnects, we pressed the panel against an edge on a jig that outlines the correct shape for the entire array. Using the jig, we configure the angle between adjacent panels, and then we use thicker interconnects for rigidly securing the panels in place (along the top and bottom of the panels). We bolted a $20 \mathrm{~mm}$ tall by $15 \mathrm{~mm}$ wide aperture to the front array. The edge of the aperture opposite the magnets should obstruct line-of-sight from the source aperture to beyond the magnets. Upon completing the assembly, we cut a length of 0.002 in thick stainless steel shim (301 series) to cover the entire length of the magnets. This shim served to protect the magnets from being contaminated by lithium.

Fig. 3.17 shows several photographs of portions of the array. The bend between adjacent panels is hardly perceptible. While quite short and thin, the lips that contain the magnets maintained a very smooth surface across all of the panels. Fig. 3.18 shows the original prototype that we had planned to build for the proof-of-principle experiment. The hardware is identical to that outlined in Fig. 3.15 except for an additional support block that includes a pair of pockets machined along its length on opposite sides. With these pockets being identical to those on the previously described support blocks, these additional blocks would allow us to stack magnet panels. The resulting material thickness between the pocket depths on these blocks (and thus the spacing between stacked panels) is just 0.030 in. We machined a few test pieces in order to verify that we could viably stack panels in this manner. We ultimately decided, however, against stacking panels mainly because these blocks were the most challenging

\footnotetext{
${ }^{27}$ We used Halbach array templates for ensuring that we installed the magnets in the proper configuration. These templates included eight magnets glued into cutouts in an aluminum plate. The magnets were arranged in a Halbach configuration and were spaced by sufficiently far to not noticeably interact with adjacent magnets. We fixed magnets to be installed in a panel in the proper configuration by placing them on top of the magnets in the template on the opposite face of the aluminum.
} 


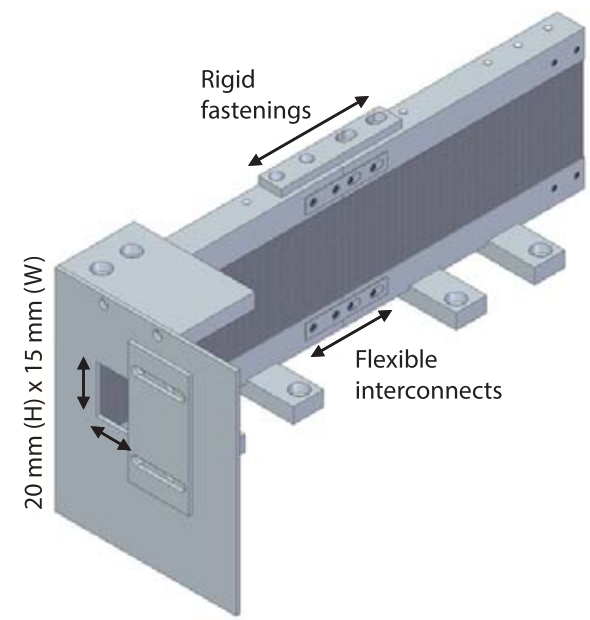

Figure 3.16: Aperture at magnet entry. Panels first link together using thin interconnects that allow the panels to bend about their adjacent edges. Upon configuring the desired angle between panels, thick interconnects fasten the panels in place. We used a monolithic jig (machined on a CNC machine) for configuring the angles between panels. After completing the array, we bolted an aperture to the front of the array. We chose the aperture height $(20 \mathrm{~mm})$ in order for the vertical extent of throughput at the end of the array to in principle match the magnet height. The aperture width was designed to be adjustible, but we ultimately made all measurements with the width configured to be $15 \mathrm{~mm}$.

pieces to machine for the assembly (and also because the planar geometry permits us to scale our measured throughput linearly with magnet height).

While we ultimately mechanically fastened magnets in place, we experimented with using vacuum compatible adhesives for making the Halbach arrays. Using epoxies present a compelling option for commercially producing arrays as we can reduce the profile of an array: we can stack magnets more densely in the vertical direction (without any gaps between layers), reduce the thickness of the assembly (requiring at most only a thin back plate), and enable atoms to more closely sample the magnet surface (as no additional components need to secure the face of the magnets). Fig. 3.19 shows the first assembly that we used for epoxying magnets into a Halbach array.

We installed magnets between a pair of 1 in thick Delrin blocks. We machined a recess into one block with its depth exactly matching the magnet thickness (requiring substantial force for pressing magnets in place). This block clamped onto the other 

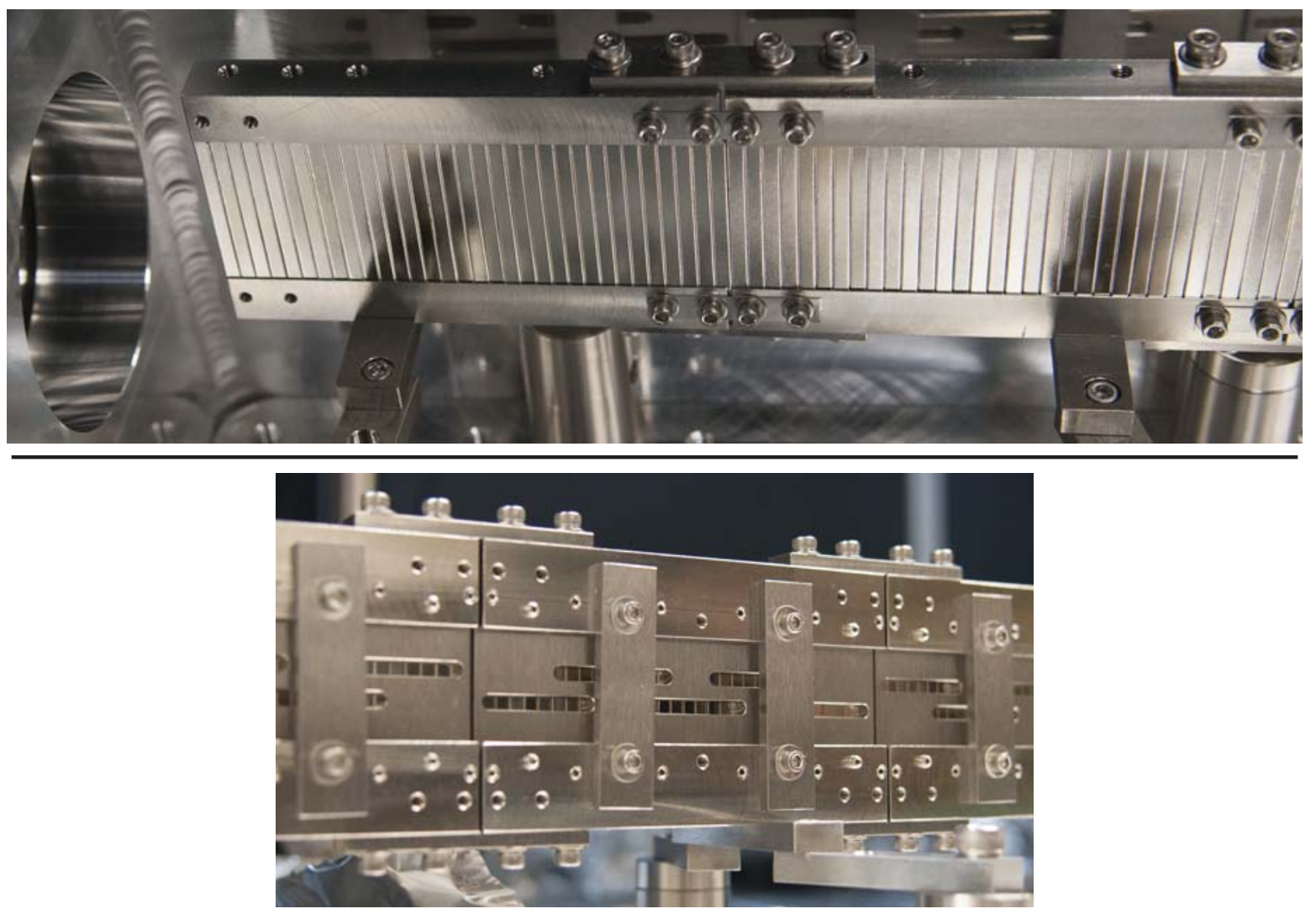

Figure 3.17: Photos of the magnet array. Each holder for the magnets almost completely exposed one face of the Halbach array while keeping the surface very smooth. We individually cleaned every magnet thoroughly using organic solvents as we could not bake-out the chamber with the magnets in place (due to concern over demagnetization).

block, with the other block including a cutout exposing the majority of one face of the magnets. We pressed the magnets against the edge of two auxiliary blocks that defined the edge of the array. Aluminum blocks bolted to both Delrin plates in order to provide stiffening. Set screws through one of these aluminum blocks pressed against its Delrin block in order to secure magnets in place. Once we installed all of the magnets into the recess, we applied epoxy to the exposed side of the magnets through the cutout on the opposite Delrin block ${ }^{28}$. We finally installed a Teflon-coated block into this cutout and uniformly pressed this block against the magnets via set screws in another aluminum plate that bolted onto the Delrin block (covering the cutout).

\footnotetext{
${ }^{28}$ We used an epoxy from Epo-Tek (part no 301-2). We chose this epoxy for its low outgassing, ability to cure at room temperature, and low viscosity.
} 


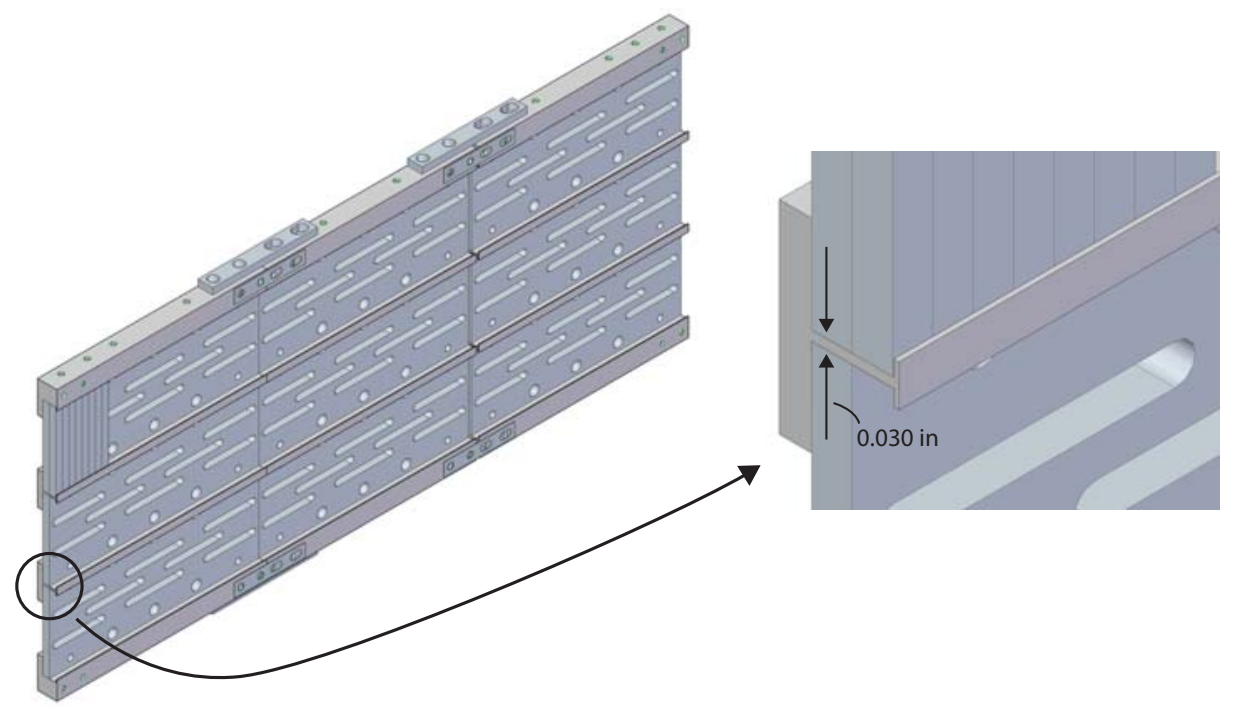

Figure 3.18: Original plans for stacking panels. We would stack panels of almost identical design to that outlined in Figure 3.15 using additional blocks that included a pair of similar pockets. These blocks would enable us to build one magnet array above another with the spacing between them given by the material thickness between the pockets (just 0.030 in).

This assembly successfully secured sixteen 0.125 in wide by 0.125 in thick by 3.5 in long magnets in a Halbach configuration, however we observed bowing of the magnets (likely due to the extended length) that led to poor smoothness across the surface and also some observable space between magnets. A better mechanism for holding the magnets in place while the epoxy cured should provide better uniformity. Crude outgassing tests using a residual gas analyzer showed substantial outgassing from the panel. Given the vacuum compatibility of the epoxy, we attribute the observed outgassing to trapped gas. Maintaining the assembly at a slightly elevated temperature should accelerate the curing and lead to more uniform filling of the gaps around the magnets.

We later tried a second, all metal tool, shown in Fig. 3.20, for epoxying magnets against a 400 series stainless steel support plate. The ends of the magnets fit under a pair of t-shaped aluminum blocks, and we applied epoxy to the exposed area of the magnets between these blocks. Two plates bolt to the support plate at positions that define the proper location for one of the t-shaped blocks. The t-shaped blocks themselves bolt to an aluminum plate that slides along the opposite side of the support plate. Spacers on 


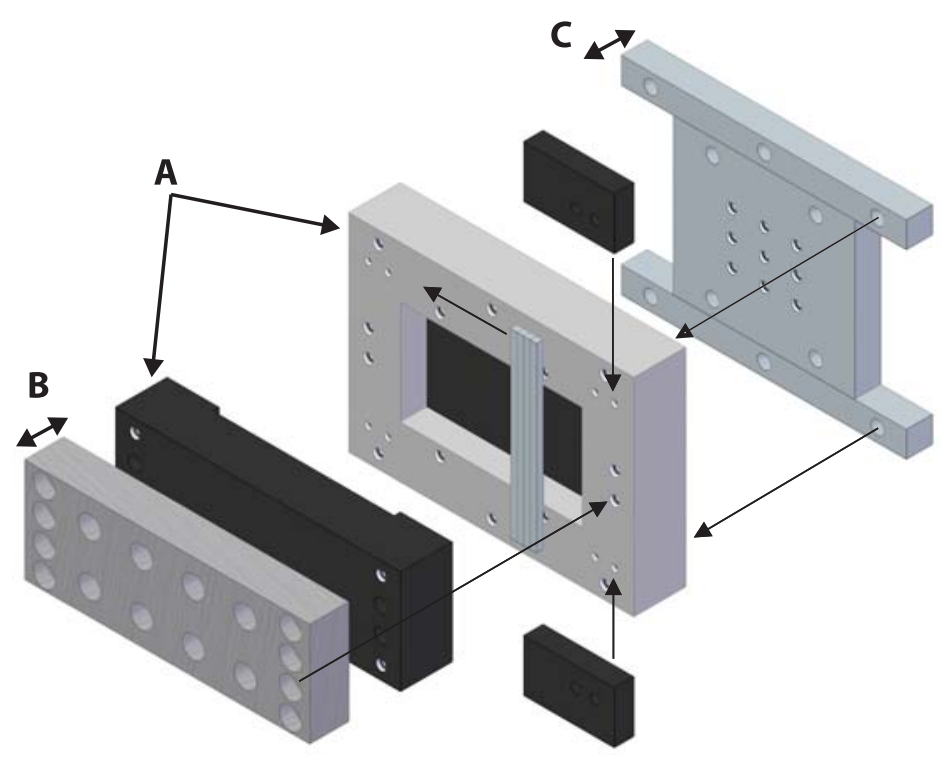

Figure 3.19: Assembly for epoxying magnets. A pair of 1 in thick Delrin blocks (A) form the core of the assembly. The magnets slide into a recess cut into one of the blocks. Set screws through an aluminum block (B) on top of this block press against the block and secure the magnets in place. We apply epoxy through a cutout on the opposite Delrin block, then press a Teflon-coated block into this cutout using another aluminum plate (C) until the epoxy cures. 


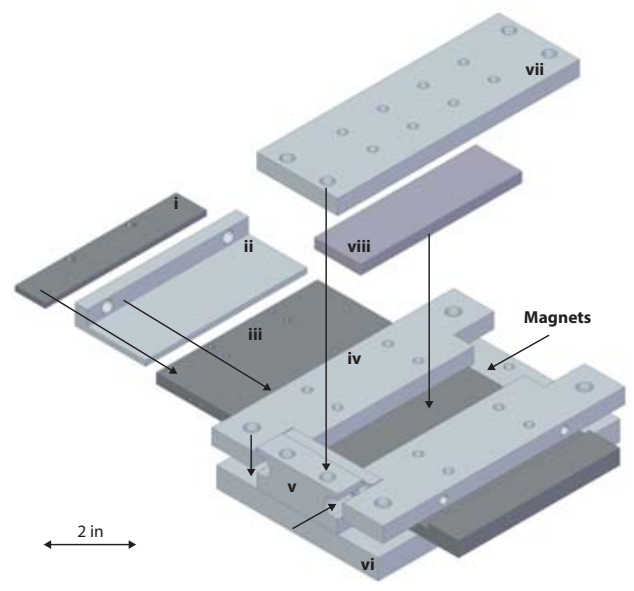

Figure 3.20: Alternative assembly for epoxying magnets. Ends of the magnets fit under a pair of t-shaped blocks (iv) on top of a slightly magnetic support plate (iii). A pair of plates ( $\mathrm{i}$ and ii) determined the location of one of these blocks, and spacers ( $\mathrm{v}$ ) fixed the gap between the blocks. We applied epoxy to the exposed area between the t-shaped blocks. Another aluminum plate (vii) pressed a Teflon block (viii) against the surface of the magnets. After magnets cured against the plate, we could add another layer of magnets to the plate by translating the t-shaped blocks and repeating the process.

this plate determine the proper spacing between the t-shaped blocks. Similarly to the previous tool, after applying epoxy we use a plate (that mounts onto the spacers) to press a Teflon piece against the magnets. After curing the epoxy at a slightly elevated temperature, the Halbach array remained in place on the suport plate. We could then repeat the process by translating the t-shaped blocks along the support plate accordingly. We ultimately stacked four layers of 32 magnets (using magnets of the same dimension as those used in the apparatus).

\subsubsection{Field Measurements}

After assembling each panel, we first checked for magnets not in the proper orientation using viewing film. We then mounted the panel onto a stage with its face parallel to the axis of a long-travel linear actuator. We measured the component of the magnetic field perpendicular to the face (z-component) along the surface of the magnets using a gaussmeter probe that we mounted onto the actuator as close as possible $(<1$ 

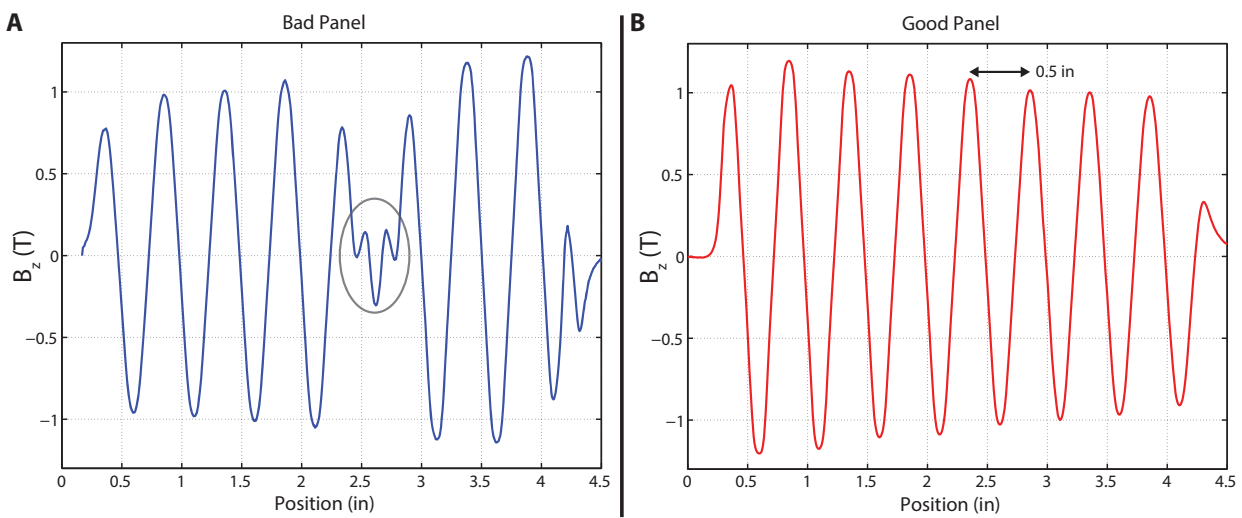

Figure 3.21: Sample field measurements. (A) A magnet with incorrect orientation of its magnetization disrupts the periodicity of the measured z-component of the field. (B) A good panel shows the proper periodicity for the z-component of the field across the entire length of the panel. The apparent reduction of amplitude across the panel length is likely an artifact of misalignment of the actuator axis with the panel surface.

$\mathrm{mm})$ to the magnets ${ }^{29}$. We first ran the probe across a panel to ensure that the probe ran close to parallel to the magnet surface. Using a stepper motor to drive the actuator, we advanced the probe by 0.00625 in between measurements over a total length of 4.375 in. Beyond confirming an estimate for the absolute field strength, we sought to verify consistent amplitude for all panels with the proper periodicity ( 0.5 in) across the length of each panel. Fig. 3.21 shows sample measurements for a pair of panels.

We installed the completed magnet array into a large coffin-like aluminum chamber with 1 in thick walls (shown in Fig. 3.22). We chose aluminum over stainless steel due to its ease of machining and lower cost. While stainless steel more readily reaches better vacuum, we felt that we could obtain suitable vacuum through proper treatment of the aluminum. Aluminum itself in fact has more favorable outgassing characteristics than stainless steel for vacuum use, however the surface of aluminum more readily holds water content that slowly outgasses. Aggressivly baking an aluminum chamber, among other things (like etching aluminum surfaces using phosphoric acid), can yield better overall outgassing than similar stainless steel chambers [76,77]. We used 1 in thick aluminum due to concern of the chamber faces bowing while under vacuum.

\footnotetext{
${ }^{29}$ We used a gaussmeter from Integrity Design \& Research Corporation (part no. IDR-329). See http://www.integritydesign.com.
} 


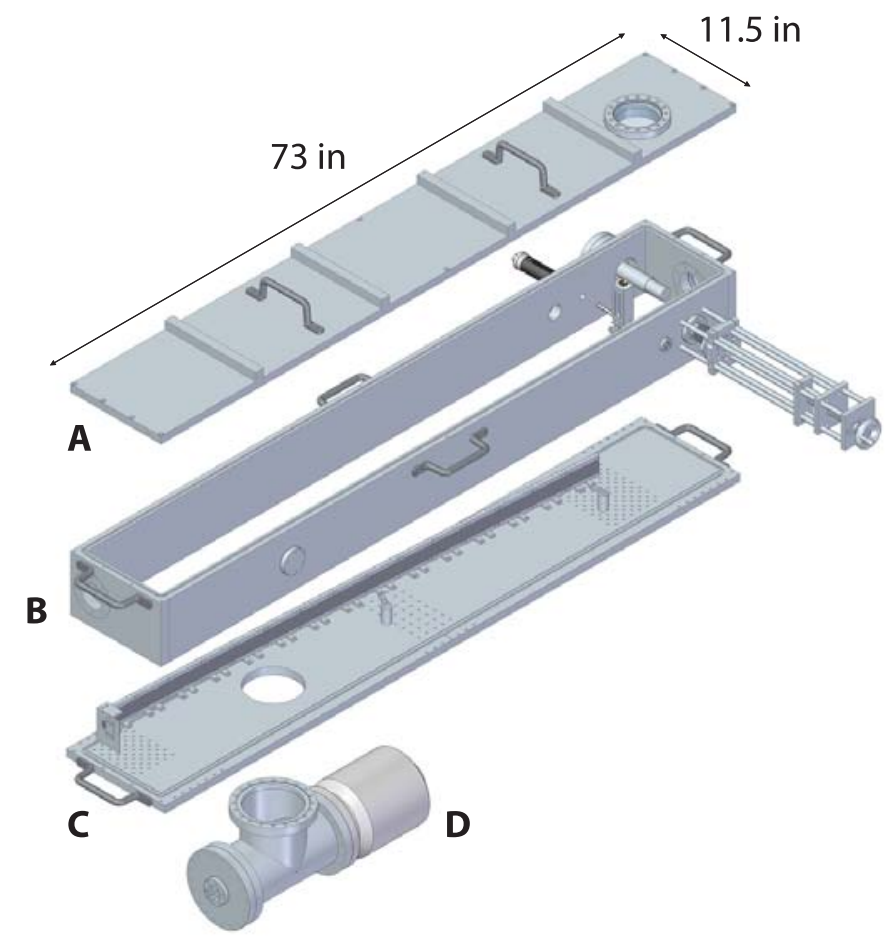

Figure 3.22: Main chamber. The 73 in long by 11.5 in wide by 8.75 in tall chamber is made entirely of 1 in thick 6061 aluminum. The chamber consists of three sections that stack on top of each other: a base plate (C), surrounding body (B), and top panel (A). Viton o-rings make seals between the sections. A $550 \mathrm{~L} / \mathrm{s}$ turbo pump maintains the pressure in the chamber at or below $10^{-7}$ Torr. The magnets mount to a hole pattern on the base plate. Detectors interface to the chamber through ports at the rear of the surrounding body.

The chamber consists of three sections: a base plate, surrounding body, and top panel. Handles bolt to the exterior of each piece for easily handling the components. The base plate bolts to an aluminum support structure that we recycled from a previous experiment. The surrounding body and top plate then mount on top of the base plate. Bolting the baseplate to the support structure provides stiffening. Likewise, bars bolted across the top plate stiffen this plate. We use custom viton o-rings that fit into grooves cut around the perimeters of the individual sections for sealing the entire chamber ${ }^{30}$.

\footnotetext{
${ }^{30}$ The cryogenics shop made our first o-rings by gluing a joint to form the proper shape. We repeatedly measured leaks on the chamber at the location of the joints on the two o-rings. We thus had o-rings made by a vendor (Marco Rubber). See http://www.marcorubber.com.
} 
All ports on the chamber interface to standard CF hardware. Due to the softness of aluminum, we could not cut knife edges on the chamber for making seals using copper gaskets. We had considered using explosion-welded aluminum-to-stainless steel transitions for making standard CF connections using copper gaskets ${ }^{31}$. To save costs, we ultimately decided to machine channels directly on the faces of the chamber that fit off-the-shelf viton o-rings for use with $\mathrm{CF}$ hardware. This solution did not work perfectly (o-rings would occasionally become distorted in making a seal by too much to be re-used), but did enable a leak rate on par with what we typically measure using stainless steel apparatus.

To position the magnet array in the chamber, we interfaced 1 in diameter optical posts to blocks that connect to the feet of the magnet panels. We fasten the posts to threaded holes in the base of the chamber by swiveling the blocks about the legs on the panels. An 8 in $\mathrm{CF}$ tee mates to a port on the base of the chamber. A $550 \mathrm{~L} / \mathrm{s}$ molecular turbopump (backed by a dual-stage rotary vane pump) connects to one port of this tee, and an ionization gauge connects to a zero-length reducer on the opposite side of the tee ${ }^{32}$. A port on the front of the surrounding body interfaces to the front-end of the apparatus. All ports on the rear of the body interface to hardware for detecting throughput.

Prior to loading the magnets into the chamber, we baked the chamber for several days at close to $90{ }^{\circ} \mathrm{C}$. This reduced the vapor pressure in the chamber to below $10^{-8}$ Torr (as indicated by the ionization gauge). After loading the magnets, we tried to minimize the number of times that we removed the top panel from the chamber. We always vented the chamber using ultra-high purity argon when breaking vacuum. Upon breaking vacuum several times, however, enough water vapor condensed in the chamber to worsen the operating vapor pressure in the chamber to $10^{-7}$ Torr. Due to the large surface area of aluminum inside the chamber, we observed extreme sensitivity of the vapor pressure to the ambient room temperature. When taking data, we used an auxiliary air conditioning

\footnotetext{
${ }^{31}$ Atlas Technologies manufactures these innovative, yet expensive bi-metal flanges. See http://www.atlasuhv.com.

${ }^{32}$ We use a Varian V551 turbo pump. Duniway Stockroom services Varian pumps.
} 


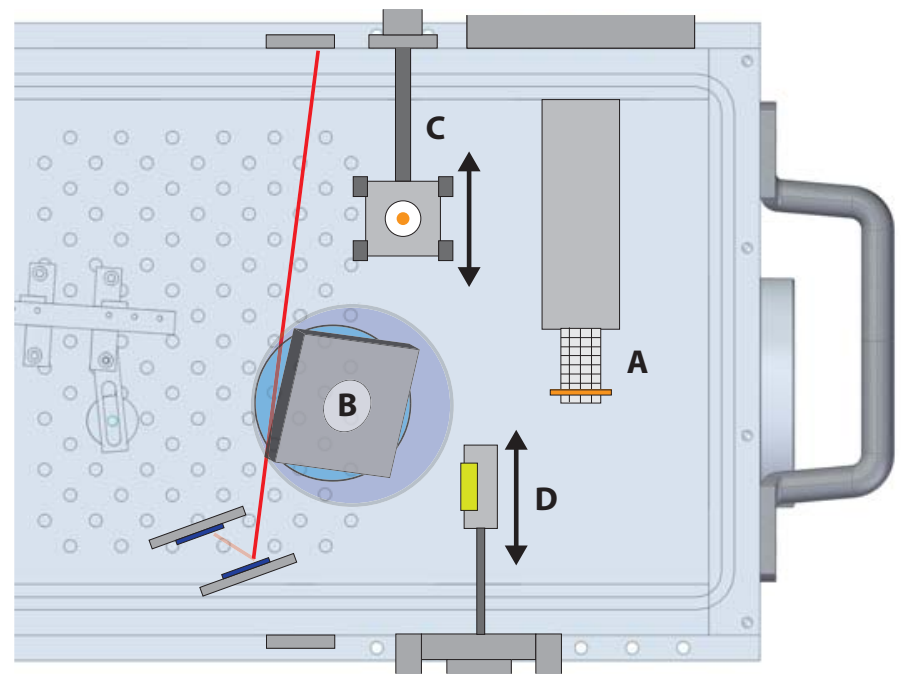

Figure 3.23: Detector layout for throughput analysis. A residual gas analyzer (A) samples the flux beyond the magnets. A collection of double-sided 4-1/2 in CF flanges position the RGA accordingly. A 3 in diameter lens achieves roughly one-to-one imaging of fluorescence onto a cooled CCD sensor (B) that sits above a 6 in viewport in the top panel of the chamber. A home-built surface ionization detector $(\mathrm{C})$ that translates via a 6 in travel linear actuator measures the spatial profile of the throughput beyond the magnets. We supplement this detector with a quartz crystal thickness monitor (D) that translates just beyond wire detector and measures deposition rates.

unit in the lab to reduce the air temperature by close to $10{ }^{\circ} \mathrm{C}$ which in turn reduced the vapor pressure in the chamber to close to $10^{-8}$ Torr.

\subsection{Detection}

Fig. 3.23 shows the armada of detectors that we use beyond the magnets for characterizing the throughput. We use a residual gas analyzer (RGA) - sampling the peak throughput beyond the magnets - and laser-induced fluorescence imaged onto a cooled CCD sensor for determining isotopic abundances in the presence and absence of optical pumping ${ }^{33}$. The RGA compares abundances of both Li-6 and Li-7 (enabling us to verify both a substantial reduction in Li-6 throughput and also no change in Li-7 throughput), although we mostly used the RGA only when loading the source with enriched Li-6. At

\footnotetext{
${ }^{33}$ We use a Residual Gas Analyzer from Stanford Research Systems. See http://www.thinksrs.com.
} 
high source temperatures while using natural lithium, the RGA indicated limited mass resolution as Li-7 contributed to the signal at mass 6, severely hindering our ability to make meaningful measurements. We therefore measured fluoresence emitted by Li-6 atoms when using natural lithium as a sensitive probe of the change in Li-6 flux in the presence and absence of optical pumping.

\subsubsection{Relative Abundances: RGA and Fluorescence}

The RGA first ionizes a fraction of incident atoms and molecules in an ionization region that consists of an iridium wire that surrounds an anode grid. The negativelybiased filament emits thermionic electrons toward the positively-biased anode grid. The voltage difference between the filament and anode provide sufficient energy for the electrons to ionize a fraction of incident molecules by way of bombardment. A repeller cage that typically surrounds the assembly enhances the electron density in the ionization region and improves the ionization efficiency. We removed this cage as lithium collected on the cage at a rate fast enough (at the highest source temperatures) to lead to an increase in our measured background over time. While removing the cage likely worsened ionization efficiency, we nearly completely mitigated this background.

A focusing plate at a negative potential extracts ions produced in the ionization region toward a quadrupole mass filter. Extensive literature exists on the principles of operation for quadrupole mass spectrometry [78]. Briefly, RF and DC voltages applied to four rods oriented symmetrically about the filter axis determine a charge-to-mass ratio that yields stable trajectories to a detector at the end of the filter. Initial calibration of the filter should provide constant mass resolution across the spectrum. In particular, the width of every mass signal should fall to $10 \%$ of its peak value across 1 amu. This compromises our sensitivity to changes in the abundance of Li- 6 beyond the magnets when using natural lithium because of the substantially higher abundance of Li-7. We can adjust the resolving power of the spectrometer at mass 6 amu by adjusting the ratio of the voltages on the quadrupole rods. Improving the mass resolution, however, leads to substantial worsening of throughput beyond the filter (due to higher likelihood for trajectories at the charge-to-mass ratio of interest to be unstable). 


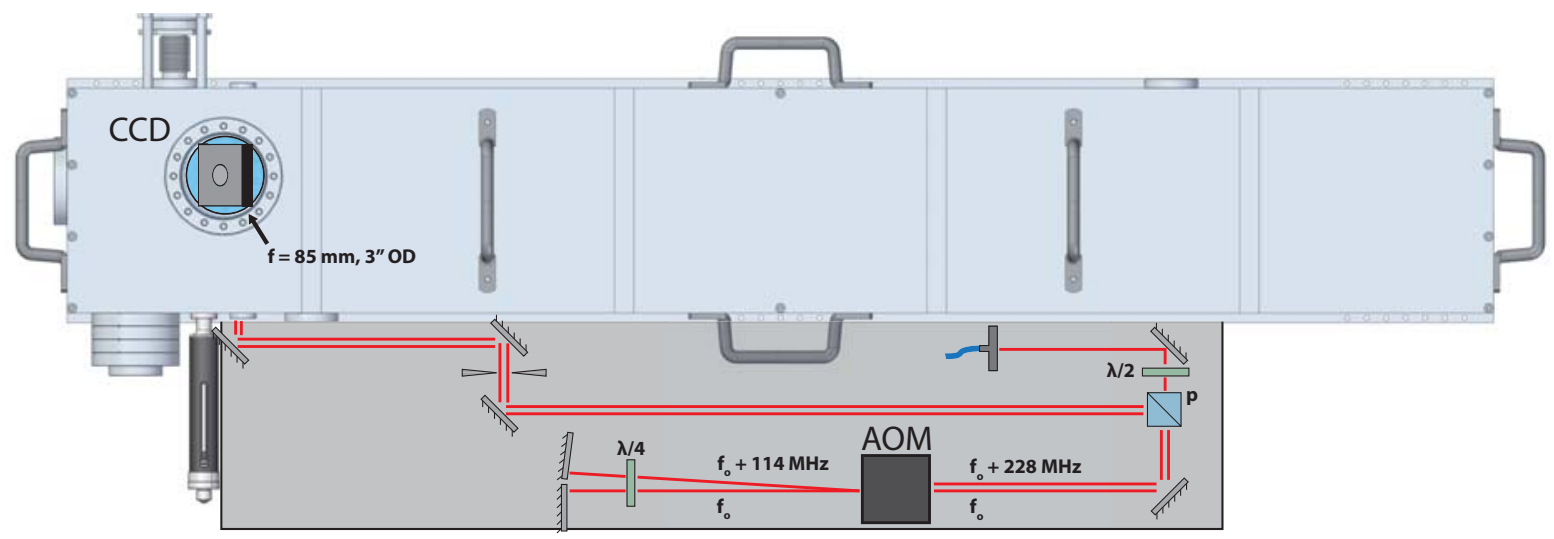

Figure 3.24: Fluorescence setup. Close to $25 \mathrm{~mW}$ from the laser setup double-passes an AOM, producing an auxiliary beam that is $228 \mathrm{MHz}$ blue-detuned from the optical pumping beam. The pair of beams (with equal powers totaling $15 \mathrm{~mW}$ over $3 \mathrm{~mm}$ diameters) pass through a $1-1 / 3$ in $\mathrm{CF}$ viewport. A 3 in lens images the fluorescence onto a cooled CCD sensor.

Detectors at the end of the mass filter include a Faraday Cup and an electron multiplier. The Faraday Cup simply monitors ion current and lacks the sensitivity of the electron multiplier to low abundances. A bias voltage on the electron multiplier accelerates ions away from the Faraday Cup onto the electron multiplier. The gain of the electron multiplier (given relative to the signal on the Faraday Cup) depends on the magnitude of this voltage. At the highest operating bias voltage, the peak gain for the RGA should exceed $10^{7}$. The gain on our unit was limited (likely due to gain degradation as a result of prior use of the RGA) to be about $1.5 \times 10^{5}$. This proved to be sufficient for most measurements of interest in this work. We did attempt to refresh the electron multiplier by cleaning via sonication in isopropyl alcohol.

Fig. 3.24 shows the optical setup for measuring Li-6 fluorescence beyond the magnets. Between 25 and $30 \mathrm{~mW}$ from the laser setup double passes an acoustic-optic modulator $(\mathrm{AOM})$, driven at $114 \mathrm{MHz}$ for generating an auxiliary beam that is bluedetuned from the frequency used for optical pumping by $228 \mathrm{MHz}$. We adjust the RF power incident on the AOM to achieve equal powers between the two beams. This auxiliary beam enhances the measured fluorescence by a substantial fraction by pumping atoms out of the $\mathrm{F}=1 / 2$ ground state. Using a quarter-wave plate, we divert the two beams toward the chamber via a polarizing beamsplitter cube. After an iris beyond the 
cube, the power of each beam is close to $7.5 \mathrm{~mW}$ over a $3 \mathrm{~mm}$ beam diameter. A 3 in diameter, $85 \mathrm{~mm}$ focal length lens sits immediately above a viewport on the top panel of the chamber. This lens achieves close to one-to-one imaging of the fluorescence onto a CCD sensor ${ }^{34}$. A narrow-line filter at $671 \mathrm{~nm}$ immediately in front of the CCD sensor drastically suppresses background. A pair of anti-reflection coated absorptive filters in the chamber suppress scattered light ${ }^{35}$. We mounted these filters to stainless steel panels that bolt to the hole pattern on the base of the chamber. The filters transmit only about $10^{-6}$ times the power of the incident light. We use a pair to absorb the fluorescence beam and also any light reflected by the first filter.

\subsubsection{Throughput: Wire Detector and Thickness Monitor}

We supplement these detectors with a commercial quartz crystal thickness monitor and a home-built surface ionization detector (or wire detector) for estimating the throughput beyond the magnets. The wire detector, shown in Fig. 3.25, is based heavily on a design from previous work [79]. Ionization of an atom on the surface of a metal occurs with substantial probability when the ionization energy of the atom is similar to the work function of the metal. The work functions of refractory metals in particular $(\Phi \sim 5 \mathrm{eV})$ are close to the ionization potential of lithium $\left(I_{L i}=5.392 \mathrm{eV}\right)$. To efficiently eject ions produced at the surface of a refractory metal, the surface must be heated to a sufficiently high temperature $(T>1500 \mathrm{~K})$ for the thermal energy acquired by the incident atom $\left(k_{B} T\right)$ to be comparable to the adsorption energy for the ion.

Our wire detector consists of a $800 \mu \mathrm{m}$ wide by $40 \mu \mathrm{m}$ thick by $7 \mathrm{~cm}$ tall rhenium ribbon. A negatively-biased $(\sim-10 \mathrm{~V})$ stainless steel collector surrounds the ribbon. Windows machined in the collector along the length of the ribbon allow the incident beam to impinge on the rhenium. We spot welded the ribbon to a pair of molybdenum tags, with one of the tags including a bend that functions as a spring that prevents the rhenium from bowing when heating [80]. These tags bolt to copper blocks that interface to current leads. Macor pieces clamp to top and bottom plates of the detector housing

\footnotetext{
${ }^{34}$ We use an Alta F47 camera (capable of operating at $-20{ }^{\circ} \mathrm{C}$. See http://www.ccd.com.

${ }^{35}$ We got the filters from ThorLabs (part no. NE60-B). See http://www.thorlabs.com.
} 


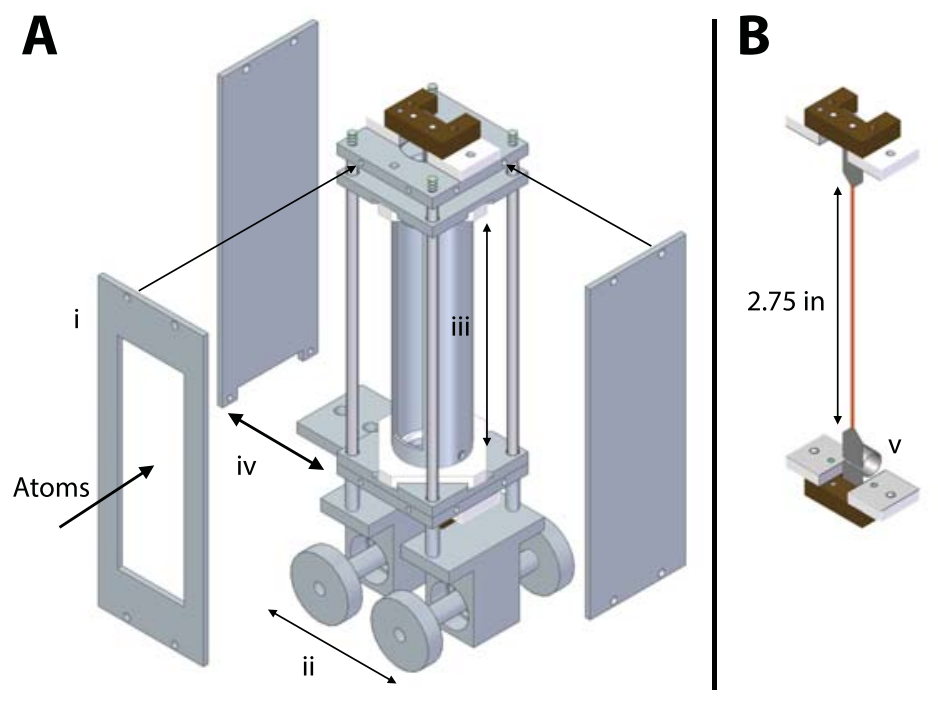

Figure 3.25: Wire detector design. (A) A stainless steel cylinder (iii) measures an ion current produced by lithium hitting a heated rhenium ribbon. Four rods and two plates define the frame for the detector. Macor pieces isolate the cylinder from the frame. The detector interfaces to a linear actuator (iv), and wheels on the detector (ii) suppress wobbling of the actuator. Three plates (i) provide radiative shielding, with the front plate including a window for the incident atoms. (B) We spot welded the ribbon to a pair of molybdenum tags (v) with one of these functioning as a spring). The tags bolt to copper blocks (isolated from the detector frame using Macor pieces) that mate to current leads. 


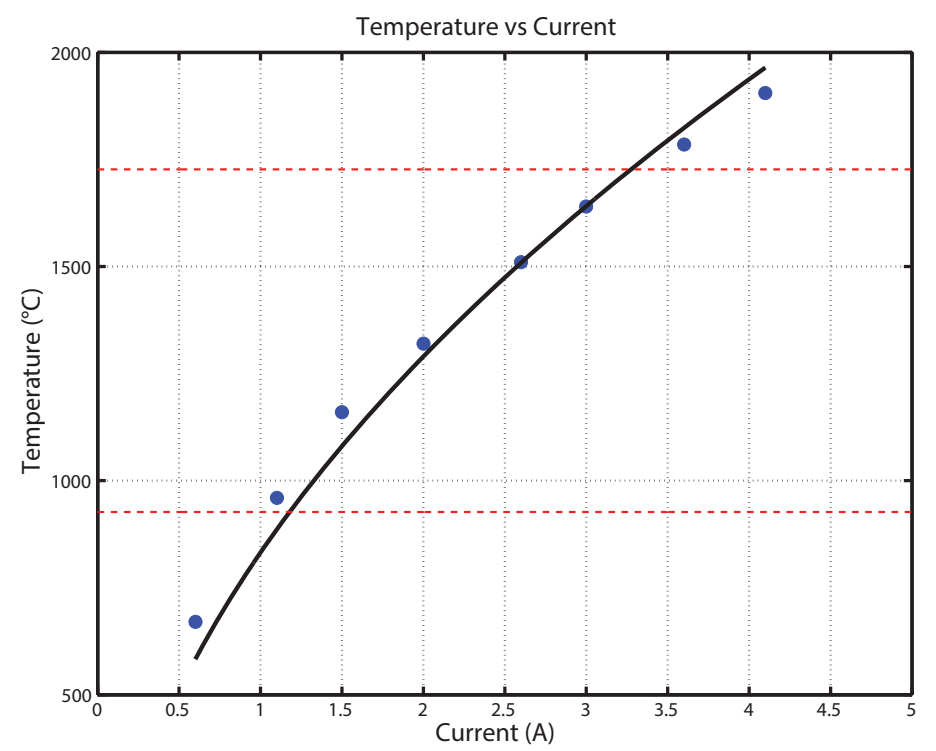

Figure 3.26: Rhenium temperature as a function of current. The blue points show measurements using an optical pyrometer. The black line shows a numerical solution to an expression describing the equilibrium temperature of the filament (as a function of current). This solution relies on fits to the resistivity and total emittance of rhenium in the temperature range given by the dashed red lines.

and isolate the copper blocks from the remainder of the detector.

Four 0.125 in stainless steel rods define the frame for the detector in tandem with the top and bottom plates. A pair of Macor plates with recesses capture the ends of the collector. These plates fit into recesses on another pair of stainless steel plates that fit onto the rods defining the frame. Screws through the top and bottom plates press against these plates to secure the collector about the ribbon. A copper block bolts directly to the cylinder for connecting a lead for measuring an ion current generated by lithium atoms hitting the ribbon. Three plates bolt to the frame of the detector for limiting radiative heating of the surrounding apparatus by the ribbon. The front plate for this housing includes a window large enough to not impede atoms from hitting the ribbon.

The bottom plate of the frame bolts onto a linear actuator with 6 in of travel ${ }^{36}$.

\footnotetext{
${ }^{36}$ We use an actuator from MDC Vacuum (part no. 660012). See http://www.mdcvacuum.com.
} 
To correct for wobble on the actuator, we built a set of wheels off of the base of the detector. Four standoffs mate to a pair of holders that contain radial bearings ${ }^{37}$. Two rods press into these bearings, and disks bolted to the ends of the rods allow the entire assembly to roll along the bottom of the chamber. A stepper motor drives the actuator. A programmable microcontroller board controls a driver for advancing the stepper motor ${ }^{38}$. We use a low-noise, variable gain transimpedance amplifier for measuring the ion current on the cylinder ${ }^{39}$.

Upon first installing the detector into the chamber, we measured the rhenium temperature as a function of current using an optical pyrometer as shown in Fig. 3.26. These measurements agree reasonably with a numerical solution to an expression for the equilibrium temperature $\mathrm{T}$ of the ribbon obtained by balancing the input power and radiative losses [79]. This expression can be written as

$$
\frac{\rho(T) I^{2}}{a b}=\epsilon(T) \sigma T^{4}[2(a+b)]
$$

where $\rho(T)$ and $\epsilon(T)$ denote the electrical resistivity and total emittance of rhenium, $a$ and $b$ are the thickness and width of the ribbon, and $\sigma$ is the Stefan-Boltzmann constant. Fits to data from literature provide expressions for $\rho(T)$ and $\epsilon(T)$ between 1200 and 2000 K [79]. Fig. 3.26 shows reasonable agreement between (3.1) and the measurements in this temperature range. For almost all measurements, we heat the ribbon to roughly $1650{ }^{\circ} \mathrm{C}$ with close to $3 \mathrm{~A}$ through the ribbon. Prior to taking data, we typically bake the ribbon at close to $1900{ }^{\circ} \mathrm{C}(4 \mathrm{~A})$ for several hours.

The wire detector enabled a lot of interesting measurements for this work. We thus adapted these designs for another wire detector for use in detecting lithium atoms in a supersonic beam. A longstanding goal of a separate experiment has been trapping then subsequent cooling of samples of hydrogen atoms produced in pulsed supersonic

\footnotetext{
${ }^{37}$ Accu-glass Products sells UHV compatible radial and linear bearings. See http://www.accuglassproducts.com.

${ }^{38}$ We use a "shield" from Adafruit for driving stepper motors that interfaces to an arduino. See http://www.adafruit.com.

${ }^{39}$ We use an amplifier from FEMTO Messtechnik GmbH (part no. DLPCA-200). See http://www.femto.de.
} 

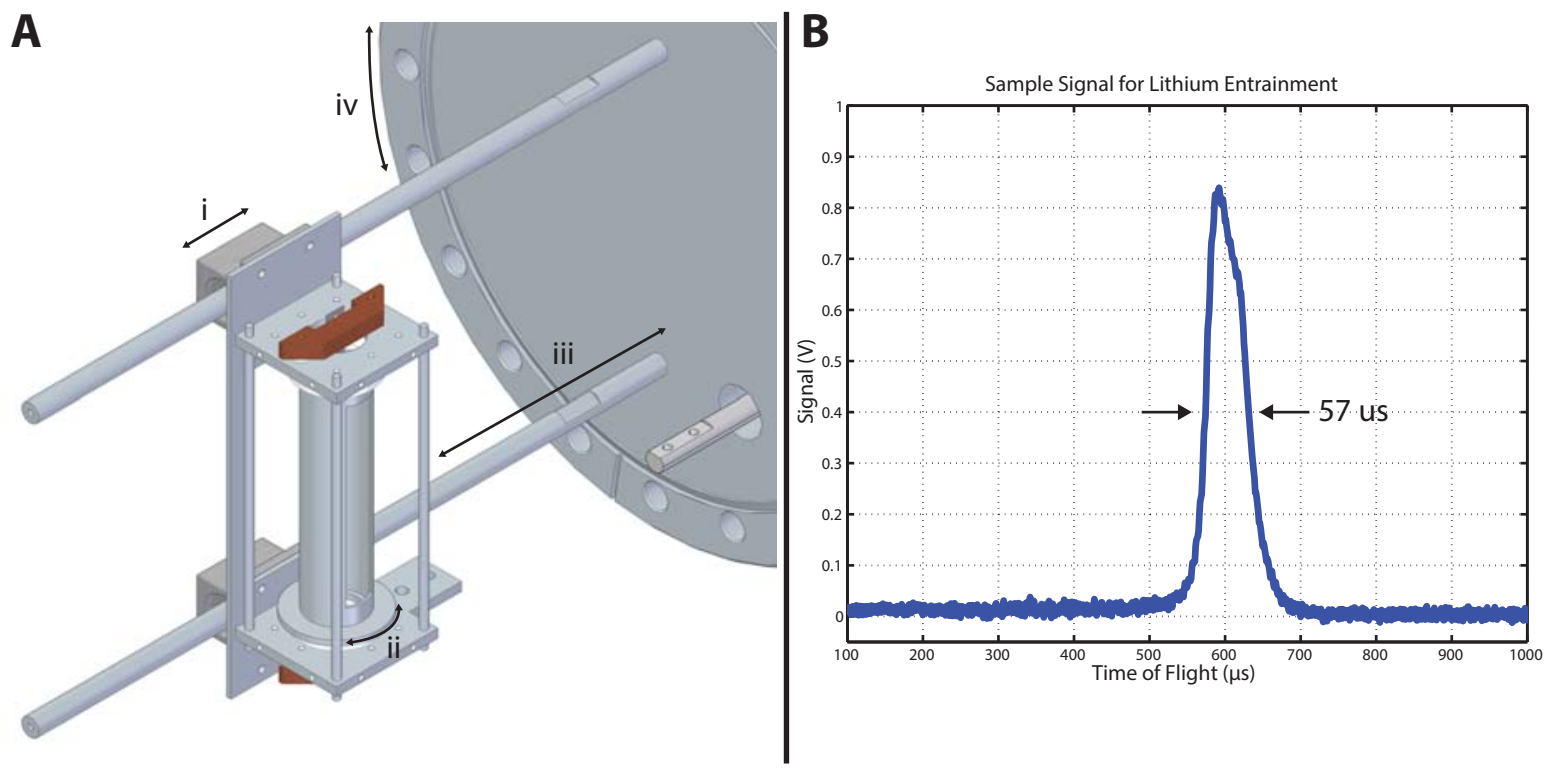

Figure 3.27: Wire detector for lithium detection in supersonic beams. (A) The entire detector interfaces to a single 8 in CF flange (iv). Linear bearings (i) connected to the detector allow the assembly to slide along rods (iii) built off of the flange. A stainless steel disc (ii) captures the collector cylinder, allowing for a lead wire to be connected more easily. (B) Sample signal produced by the wire detector as a function of time (with the initial time corresponding to opening the pulsed valve).

beams [81]. This apparatus uses a sequence of pulsed electromagnetic coils, called an "atomic coilgun", for bringing a fast-moving supersonic beam of paramagnetic atoms to rest. Recent work has focused on co-trapping lithium and hydrogen atoms, then further cooling the lithium atoms for sympathetically cooling the hydrogen atoms. Lithium should drastically facilitate this cooling due to a substantially higher elastic collision cross-section for a hydrogen and Li-7 atom (over a collision between two hydrogen atoms) $[82,83]$.

The first step of this work has focused on entraining lithium atoms into a supersonic beam by using $50 \mathrm{~mJ}$ pulses from a Q-switched Nd:YAG laser at $1064 \mathrm{~nm}$ for ablating a lithium target positioned just beyond a pulsed valve. Fig. 3.27 shows modifications to the wire detector design and also sample measurements indicating lithium entrainment into a supersonic beam. Key changes to the detector include simplifying all Macor pieces (for either reducing machining time or allowing the use of off-the-shelf components like washers and screws), building a stainless steel disk with a recess for 
capturing the collector and more easily connecting a current lead, and attaching linear bearings to the housing for smoother motion. An 8 in CF flange contains the entire detector (including electrical feedthroughs), simplifying assembly and installation.

Inferring the throughput using just the wire detector relies on a valid calibration for both the ionization and detection efficiency of the detector. Rather than rely on a subjective calibration, we supplemented wire detector measurements with deposition rates measured using a quartz crystal thickness monitor ${ }^{40}$. The thickness monitor applies RF voltage across a quartz crystal at a frequency corresponding to an electromechanical resonance of the crystal (intially at $6 \mathrm{MHz}$ for our sensor). As mass builds up on the face of the crystal, this resonance frequency lessens in a repeatable and precise manner.

A simple expression relating the thickness of a film $T_{f}$ on the sensor to the change in resonance frequency $\Delta f$ is given by

$$
T_{f}=\frac{K(\Delta f)}{\rho_{f}},
$$

where $K$ is a function of crystal properties and $\rho_{f}$ denotes the film density. Subsequent work has optimized features of the quartz crystals (particularly those determining the response spectrum of the crystals) and enabled more precise determination of film thicknesses [84]. An important consideration when using thickness monitors is that other experimental fluctuations - notably including the crystal temperature - can alter the crystal resonance frequency. We therefore maintain water cooling on the sensor using a chiller dedicated to the thickness monitor. We keep the water lines from the chiller to the sensor as short as possible, and we insulate them heavily using foam to suppress sensitivity to changes in the room temperature.

We translate the thickness monitor sensor across the collection plane beyond the magnets just beyond the wire detector. We built a translation stage, shown in Fig. 3.28, for precisely positioning the sensor using a bellows. This stage frame consists of four long 0.5 in diameter optical posts that screw directly into a hole pattern on the side

\footnotetext{
${ }^{40}$ We use a thickness monitor from INFICON (including their Q-Pod transducer, sensor, and quartz crystal). See http://www.inficon.com.
} 

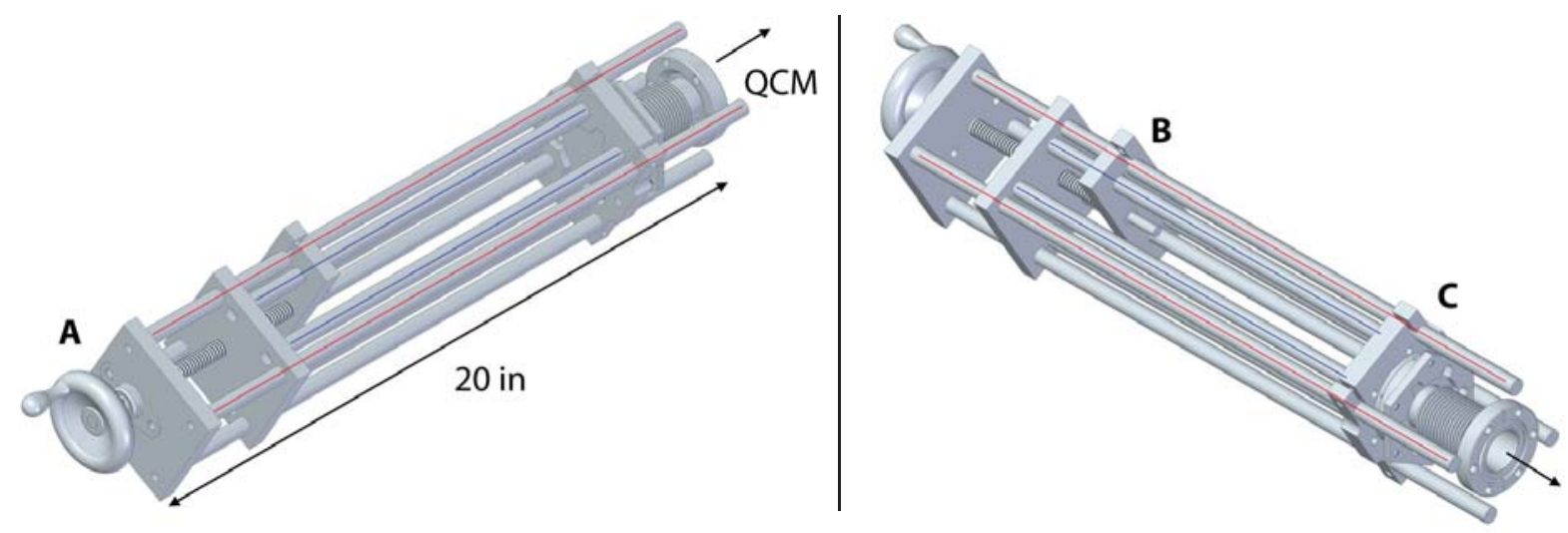

Figure 3.28: Thickness monitor translation mechanism. The frame consists of a rear plate (A) that bolts to four optical posts (red) that thread into the chamber wall. Two plates (C) clamp around the flanges for the thickness monitor and an edge-welded bellows. One of these plates fits over the rods defining the frame. Four rods (blue) connect this plate to another plate that threads onto a threaded rod and also slides along the frame. We turn the threaded rod using a handwheel in order to move the thickness monitor along the frame. An auxiliary plate (B) prevents the threaded rod from wobbling.

of the chamber body surrounding the port for the thickness monitor. The thickness monitor is mounted through a flange to a 2-3/4 in CF edge-welded bellows whose other end mates to the port on the chamber. A pair of aluminum plates clamp around the thickness monitor and bellows flanges with one of these plates including holes that slide along the stage frame. Four rods interconnect this plate to another plate (also sliding along the frame rods) that threads onto a 1/2-13 threaded rod with machined ends. The end of the threaded rod fits through a flanged radial bearing that mounts to a plate that connects to the end of the rods defining the frame. A handwheel then turns the threaded rod, actuating motion of the bellows (and thickness monitor) along the frame. This stage allows repeatable positioning of the sensor to within less than $0.005 \mathrm{in}$.

Fig. 3.29 summarizes the entire apparatus. The beam line leading up to the magnets, including the source, heated aperture, optical pumping section, and relevant optics, pivot about the magnet aperture. A linear actuator and a pair of micrometers allow us to control the incidence angle of the beam onto the magnets to within a few mrad. A large aluminum chamber contains the magnet array and detectors. The array 
consists of fifteen panels bent over a length of $1.5 \mathrm{~m}$ by just $20 \mathrm{mrad}$. An aperture at the front of the array obstructs line-of-sight from the heated aperture to beyond the magnets (for certain incidence angles). We characterize throughput using four detectors: an RGA and laser-induced fluorescence provide isotopic selectivity (for relative abundance measurements), and wire detector and thickness monitor allow us to estimate throughput. 


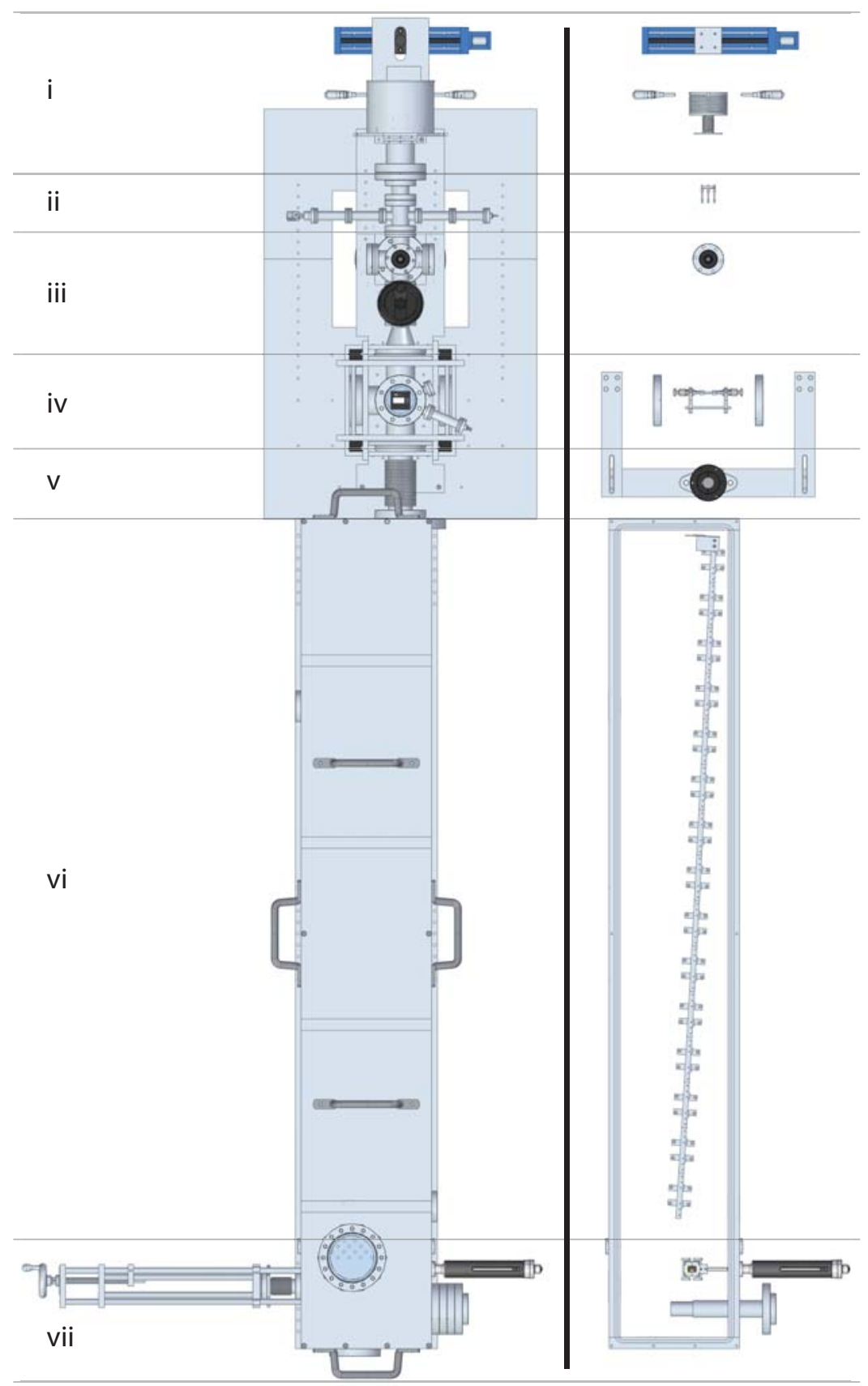

Figure 3.29: Complete apparatus. The beam line consists of the source (i), heated aperture (ii), beam blocking flag and gate valve (iii), and optical pumping cross and Helmholtz pairs (iv). A bellows (v) interfaces the beam line to the main chamber (vi). Four detectors (vii) allow us to characterize both the purity and abundance of the throughput. 


\section{Chapter 4}

\section{Measurements}

\subsection{Maximizing Efficiency}

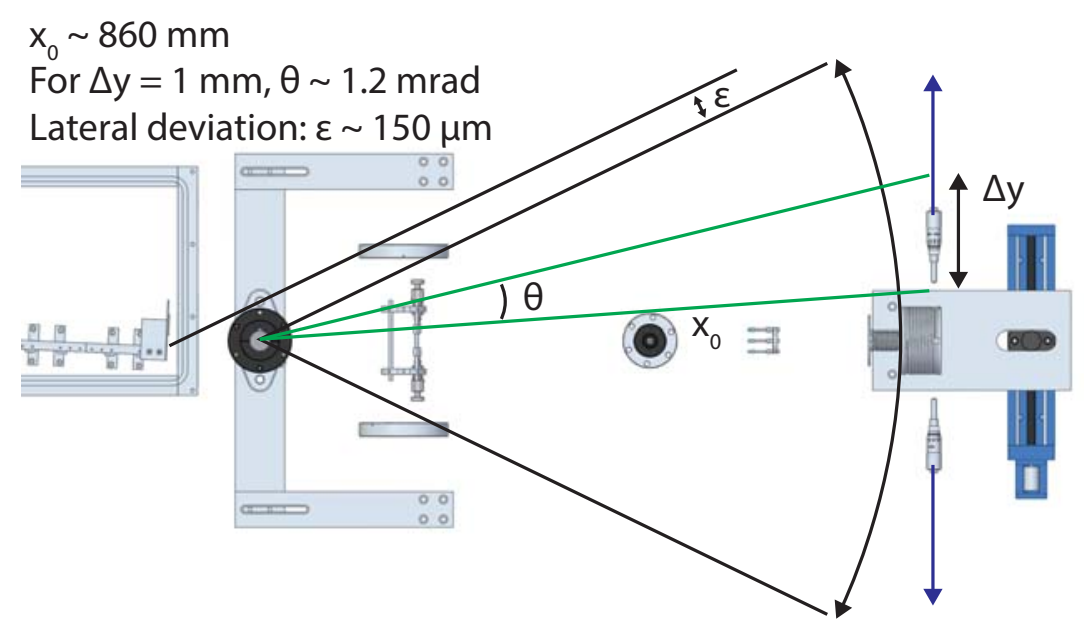

Figure 4.1: Calibration for rotation angle of atomic source. A given micrometer displacement corresponds to a rotation angle for the beam line (governed by the apparatus geometry). With the beam line not pivoting actually about the magnet aperture (instead about the center of the bellows), we must apply a correction for the effective rotation about the aperture.

Prior to any purity or flux measurements, we optimize the incidence angle of the thermal beam onto the magnet array by rotating the beam line about the bellows interfacing to the guide chamber as shown schematically in Fig. 4.1. We dial in a rotation angle for the beam line by offsetting the micrometers accordingly, then displacing the plate connecting the beam line assembly to the linear actuator until it makes contact with the relevant micrometer. For a $1 \mathrm{~mm}$ offset of a micrometer, the beam line should rotate by roughly $\sim 1.2 \mathrm{mrad}$. In fact, the assembly rotates the beam line about the center of the bellows connecting the beam line to the magnet chamber i.e. not the magnet aperture). Rotating the beam line thus technically displaces the beam on the 

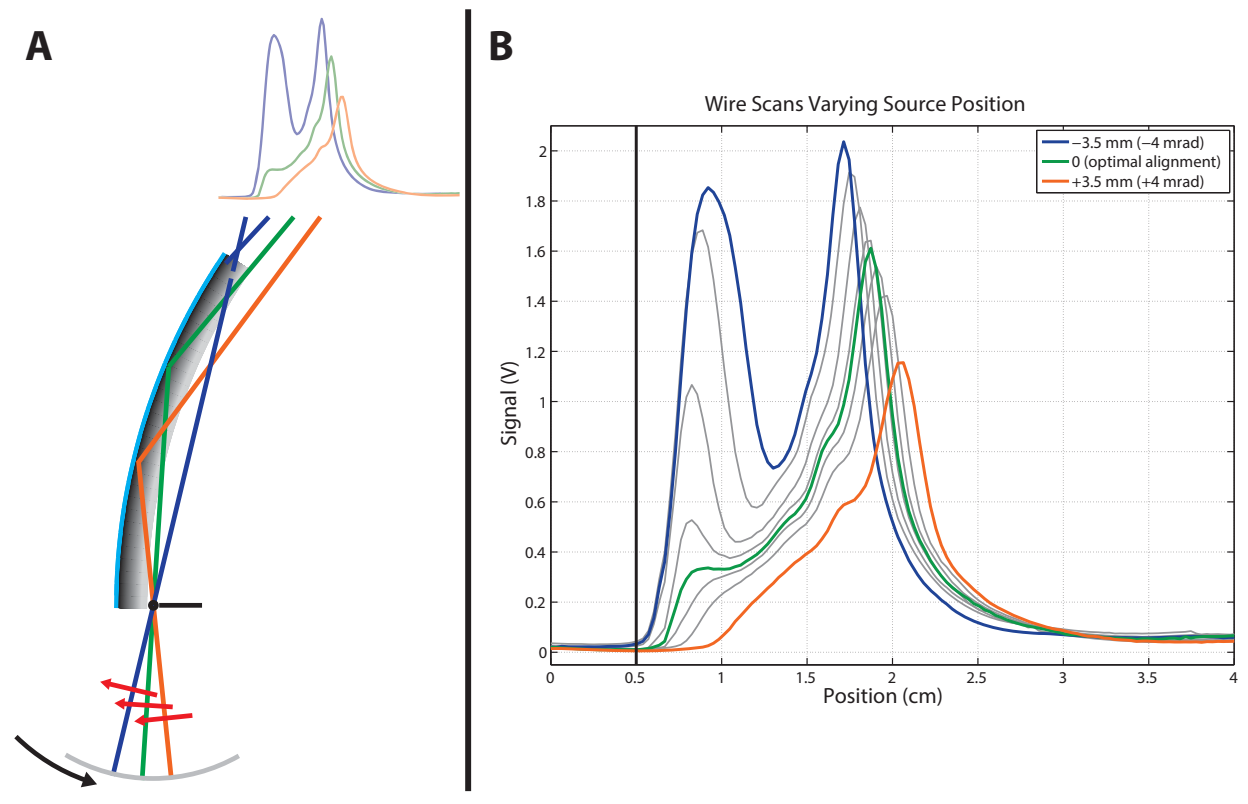

Figure 4.2: Maximizing apparatus efficiency via wire detector. (A) Translating the source closer to granting line-of-sight past the magnets yields higher throughput (orange then green), however moving too far (blue) grants sight past the magnets thus yielding a second observed peak in the throughput corresponding to undeflected atoms. (B) Actual wire traces corresponding to different source positions (colored traces corresponding to representative cases in $(\mathrm{A}))$.

magnet aperture (in addition to tweaking its incidence angle). Due to the small distance between the center of the bellows and the magnet aperture (compared to length of the beam line), this displacement is less than $150 \mu \mathrm{m}$ for a $1 \mathrm{~mm}$ offset of a micrometer.

Moving the source too far to the left (in accordance with the orientation given in Fig. 4.2(A)) grants line-of-sight from the source to the collection plane and reduces purity. In contrast, translating the source too far to the right chokes off throughput: the far edge of the magnet aperture progressively obstructs more of the magnet surface, and velocity components perpendicular to the face of the array increase, thus decreasing the maximum velocity for trajectories that can be reflected. The optimal position for the source is the closest attainable position to allowing line-of-sight beyond the magnets that does not actually grant line-of-sight. In this location, the apparatus should yield complete suppression of Li-6 (assuming perfect optical pumping) while maximizing Li-7 throughput past the magnet barrier. 
We use traces that we measure with the wire detector for locating this optimal position. Fig. 4.2(B) shows a collection of traces obtained by pivoting the source about its optimal location. For all traces we displace the wire detector by $1.75 \mathrm{in}$. The distance between steps varies for certain measurements, but for most traces we advance by 0.0125 in between steps (corresponding to half a revolution of the stepper motor driving the actuator). At every position we sample a large number of measurements (typically 100) whose average we record as the signal at that position. We always immediately follow recording a trace corresponding to certain experimental conditions by measuring a background trace with the flag blocking the atomic beam. We typically measure the ion current after a transimpedance amplifier (with a $10 \mathrm{~Hz}$ filter) whose gain we set to $10^{8} \mathrm{~V} / \mathrm{A}$.

At all positions granting line-of-sight, the wire traces in Fig. 4.2(B) show a pair of peaks. One of these peaks corresponds to straight-line trajectories from the source to the collection plane, while the other corresponds to trajectories that reflect off of the magnet array. As the incidence angle of the beam line moves away from granting line-of-sight, the line-of-sight contribution to the throughput lessens. As this contribution reduces, however, its cutoff remains at close to the same position on the traces. This position corresponds to the shadow of the last magnet on the array. Using the array geometry, we can infer the lateral offset between the last magnet and the flux. While rotating the source away from line-of-sight, the peak throughput shifts away from the magnets as larger incidence angles on the magnets yield larger outgoing angles after trajectories (among slower atoms) reflect from the magnets. After completely suppressing line-ofsight, the edge of the throughput begins moving away from the magnets, showing the shadow of the edge of the aperture at the magnet entrance.

At the optimal position shown in Fig. 4.2(B), we had already offset a micrometer by $23.5 \mathrm{~mm}$ (toward granting line-of-sight), corresponding to rotation of the beam line by $27 \mathrm{mrad}$. This necessary offset suggests slight misalignment between the beam line and magnet array. While we used a meticulous procedure for aligning the beam line to the chamber containing the magnets, we somewhat coarsely oriented the magnets in the chamber. As shown later, however, the magnet aperture comfortably fit inside the 
atomic beam diameter despite this offset.
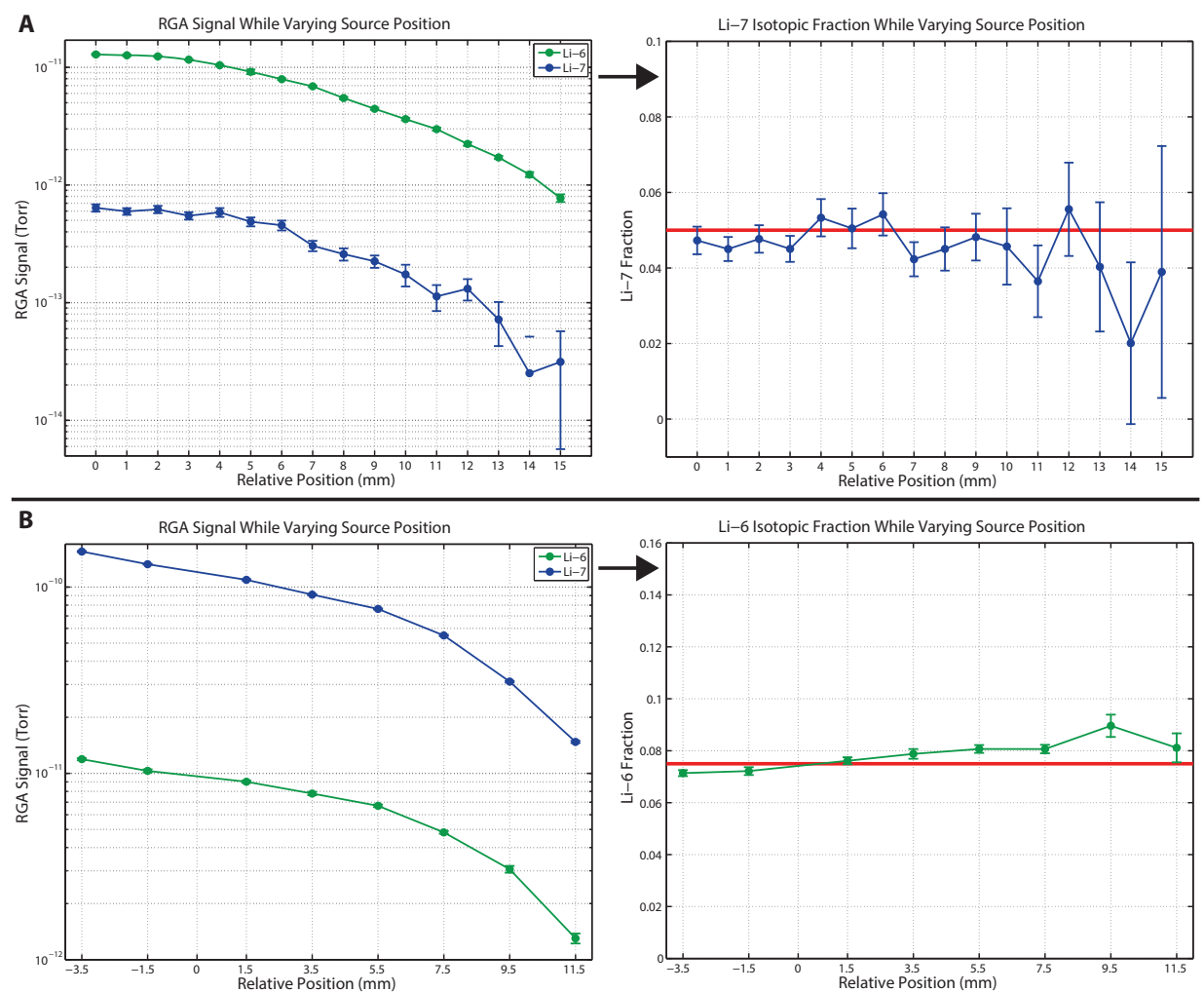

Figure 4.3: RGA signal dependence on source position. (A) RGA signals at 6 and 7 amu (and isotopic fraction) using enriched Li-6 in the source (G1) at $500{ }^{\circ} \mathrm{C}$. The position at $0 \mathrm{~mm}$ corresponds to the optimal position for the source, and positive displacement corresponds to moving the source away from line-of-sight. (B) Similar signals using natural lithium in the source (G3) at $650{ }^{\circ} \mathrm{C}$.

As shown in Fig. 4.3, we similarly measured throughput (with isotopic sensitivity) on the RGA as we translated the source. With the RGA at a fixed position, we only sampled the throughput on the collection plane. These measurements (when compared to wire traces) allowed us to position the RGA using double-sided $4-1 / 2$ in CF flanges such that the ionization region sampled the collection plane between 1.5 and $2 \mathrm{~cm}$ (as defined on Fig. 4.2). In particular, we looked for the measured signals on the RGA at masses 6 and 7 amu to monotonically increase upon moving the source toward granting line-of-sight (as the overall throughput across the plane increases and the peak signal shifts closer to the magnet shadow). Using the RGA, we could verify the relative abundances of Li-6 and Li-7 when using both enriched Li-6 and natural lithium in the 
source. At most oven positions, the Li-7 fraction when using enriched Li-6 in the source is consistent with its nominal value of $5 \%$. Likewise, when using natural lithium in the source, the Li-6 fraction is close to its natural value of $7.5 \%$.

For subsequent analysis (particularly when quantifying throughput), we needed to verify that the shape of the profile did not change appreciably while increasing the source temperature at a fixed source position. Fig. 4.4 shows wire traces (using natural lithium) as a function of source temperature with the source close to its optimal position. Scaling all traces accordingly shows that the shape of the throughput is roughly constant except at the peak temperature $\left(750^{\circ} \mathrm{C}\right)$. At this temperature, the distribution across the trace has changed, with lower flux at the peak and higher throughput near the magnet shadow. This change is consistent with the source output transitioning from effusive flow to flow with properties governed by gas dynamics (i.e. supersonic flow). A narrower, faster velocity distribution incident on the magnet aperture should yield higher throughput adjacent to the magnet shadow and lower flux at the peak of the profile. More trajectories should be incident on the end of the array at glancing angles. Likewise, those (fewer) trajectories occupying the relevant subset of the angular distribution incident on the magnets that contributes to the peak of the throughput profile will have higher speeds that reflect less efficiently. Nonetheless, with the shape of the profile not changing up to $750{ }^{\circ} \mathrm{C}$, we can safely use the shape of any of the traces up to that temperature for deriving throughput at a given temperature (as discussed later)

Using the piezo-actuated variable slit that we described previously, we can correlate trajectories in the pumping region to features on the throughput beyond the magnets as shown in Fig. 4.5. We initially opened the slit to a width of 0.375 in in order to not obstruct any trajectories. Upon closing this window by just 0.055 in on the side closest to the magnets, we observe suppression of throughput away from the magnets. This slit edge likely suppresses the slowest trajectories producing the highest reflection angles away from the magnet array. We reduce the width by displacing the other edge of the aperture by more than 0.200 in before suppressing throughput adjacent to the magnet shadow. Trajectories originating from this side of the cross likely include the atoms impinging on the magnets at the steepest angles. With the aperture edges frequently 

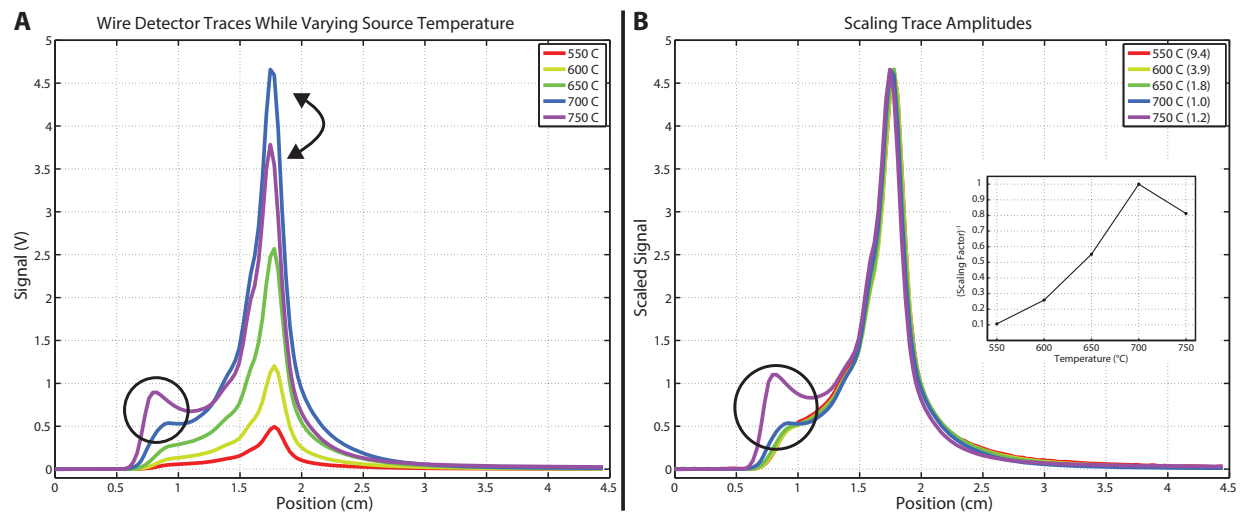

Figure 4.4: Wire detector traces as a function of source temperature. (A) Raw traces showing the scaling while increasing source temperature. We measured traces using natural lithium in the source (G3) with the source close to its optimal position. (B) The shape of the throughput remains constant at up to $750{ }^{\circ} \mathrm{C}$. The inset shows the scaling factors that we apply to each trace for matching the amplitude of all traces.

stalling, we removed the assembly from the apparatus after determining that the atomic beam width in the pumping cross contributing to throughput beyond the magnets is close to 0.150 in. 

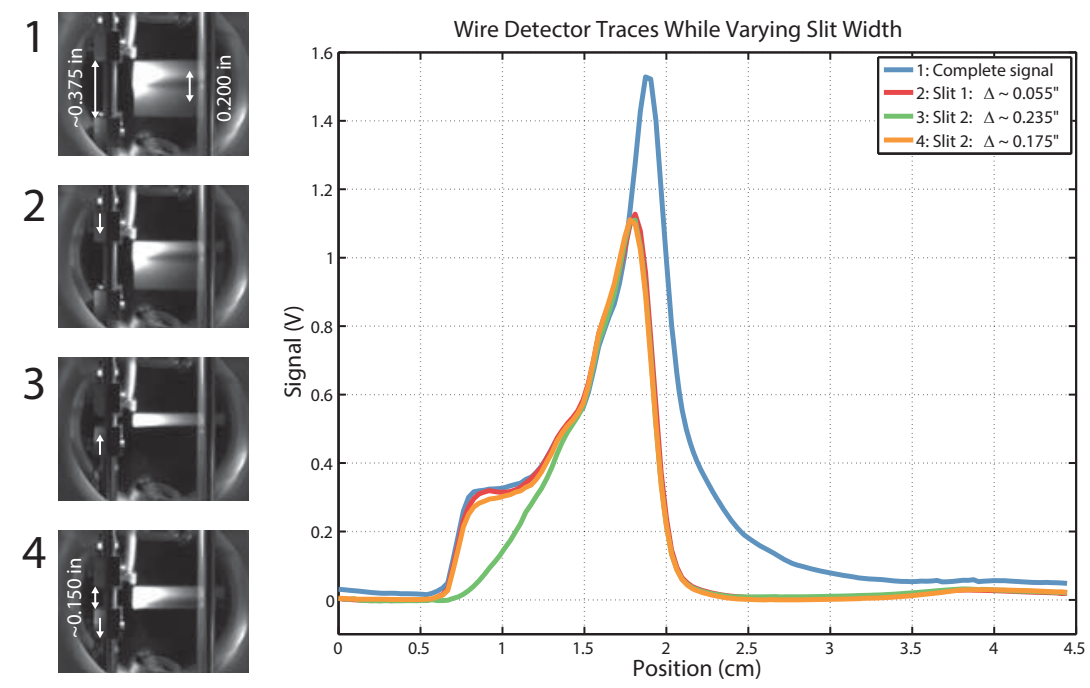

Figure 4.5: Determining relevant spatial extent of atomic beam in optical pumping chamber. Images show the slit configurations corresponding to the wire traces. These images show the fluorescence (heavily saturated) produced by the optical pumping beam (imaged onto a CCD). 


\subsection{Throughput and Efficiency}

While the wire detector provides the spatial profile of throughput beyond the magnets, we lack calibration for both its ionization and detection efficiencies. The SahaLangmuir law gives an expression for estimating the ionization probability of an atom incident on a metal surface. The expression depends on surface temperature, ionization potential of the atom, and work function of the metal (with the latter being a function of temperature). Prior work (derived from both calculations and measurements) suggests large changes in ionization probability over a few hundred Kelvin, with the probability at a particular temperature being highly sensitive to overall vapor pressure in proximity to the surface ${ }^{1}[79]$. Moreover, measuring the ionization probability would require a priori knowledge of the flux incident on the wire. To infer the detection efficiency, we must estimate the collection efficiency for ions onto the collector (in addition to the ionization efficiency). We expect this efficiency to be close to unity (given the potential difference between the ribbon and collector), although a plausible estimate requires us to consider factors including the collector geometry and energy distribution for ions emitted from the ribbon.

As summarized by Fig. 4.6, rather than attempting to derive the flux using estimates for the ionization and detection efficiencies of the wire detector, we derive a calibration for the wire scans using thickness monitor measurements at various locations across the collection plane. In contrast to the wire detector, the thickness monitor directly measures flux as a deposition rate over its $8.25 \mathrm{~mm}$ sensor area. As the extent of the throughput $(\sim 2-2.5 \mathrm{~cm}$ exceeds the sensor diameter we must translate the sensor across the plane beyond the magnets in order to sample all of the flux. Moreover, as indicated by Fig. 4.2 the flux can vary by a large amount across the sensor area depending on the sensor position. For instance, were we to position the sensor with one edge at the location yielding peak flux for the green trace in Fig. 4.2 and the other edge further away from the magnets, the flux would reduce by over $90 \%$ across the sensor.

\footnotetext{
${ }^{1}$ The ionization probability appears to be particularly sensitive to the partial pressure of oxygen. In our case, given the high operating temperature for the ribbon and low vapor pressure in the magnet chamber, we suspect our ionization probability to be less than $1 \%$ based on previous work by Delhuille, et al.
} 


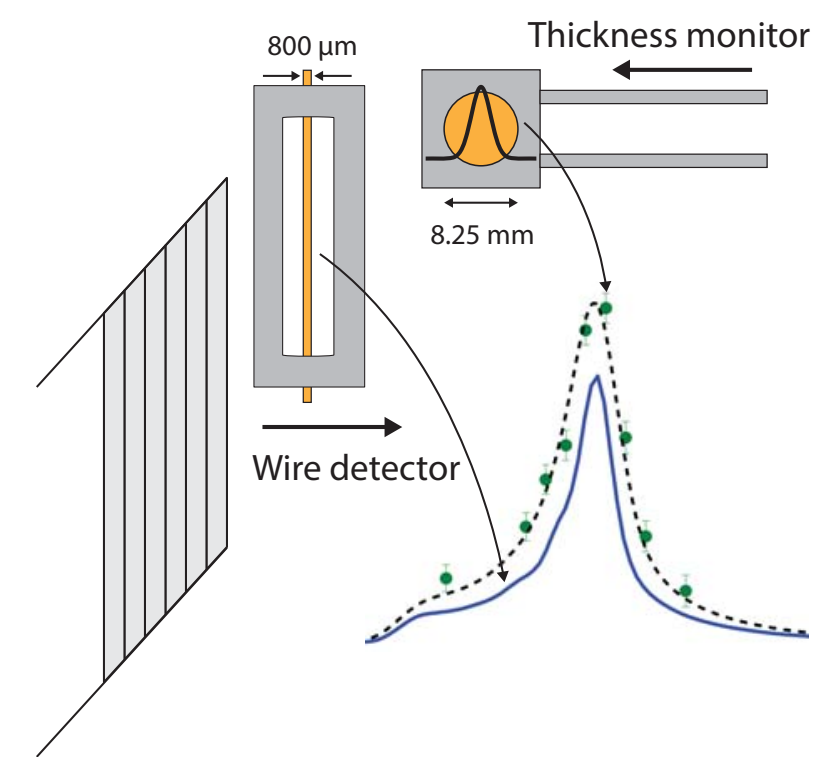

Figure 4.6: Overview of procedure for estimating throughput. We first measure a response function for the thickness monitor (black trace overlaying the thickness monitor) with the thickness monitor in the pumping region. We then identify a calibration factor for a wire trace (blue) that reasonably approximates the thickness monitor data (dashed black curve is calculated and green points are data) when taking convolutions with the response function over the sensor area. With this factor we can integrate the wire trace to get a throughput number.

A particular thickness monitor measurement corresponds to the convolution of the flux per unit width across the sensor area with a function specifying the responsivity of the sensor per unit width across its diameter. A wire trace gives the functional form (lacking calibration) for the flux per unit width across the collection plane. By overlaying a set of deposition rates and a wire trace, we can reasonably infer the location of the sensor for a given measurement along the profile. The response function must weigh the flux per unit width across the sensor by the fractional area of the sensor per unit width that fits within an enclosing square (of edge length matching the sensor diameter). As illustrated by prior work, however, we cannot assume uniform mass sensitivity of the sensor across its surface [85]. In fact, the variation in mass sensitivity across a quartz crystal resonator closely matches the change in vibration amplitude across the sensor.

In order to directly incorporate the circular shape and non-uniform mass sensitivity into our measurements, we directly measured an overall response function - shown 

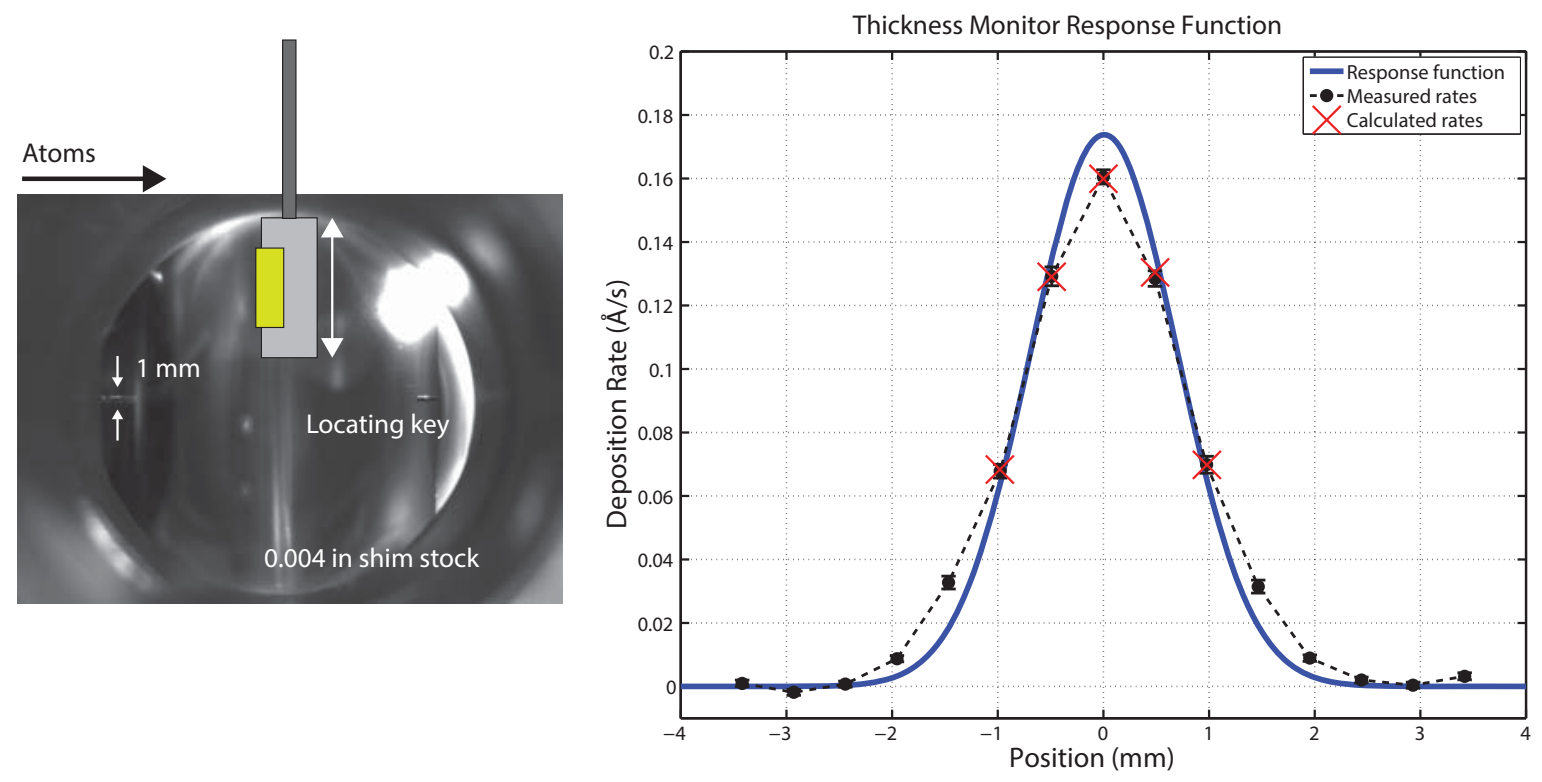

Figure 4.7: Response function measurement. The black data points show deposition rates obtained by translating the thickness monitor across a $1 \mathrm{~mm}$ wide slit in the optical pumping region. With these measurements, we obtain a Gaussian curve (blue trace) whose convolution with a $1 \mathrm{~mm}$ step function at the position corresponding to any data point (red crosses) reasonably reproduces that data point. 
in Fig. 4.7 - for the thickness monitor by first translating the sensor immediately behind a $1 \mathrm{~mm}$ wide aperture with a vertical aspect ratio (with height exceeding the sensor height) in the pumping cross where atomic flux is substantially higher and more uniform across a larger width. We machined the aperture on a piece of 0.004 in stainless steel shim stock. We simply rolled the shim to a diameter small enough to slide along the arm in the cross for optical pumping. Upon releasing the shim (with the aperture at the center of the pumping region as determined using a CCD), the material un-rolled and remained in place against the cross tube.

For every thickness monitor measurement, we sample material over a duration (typically several minutes for measurements in the pumping region and tens of minutes for positions corresponding to lowest throughput beyond the magnets) long enough to accumulate several Angstroms (for the sake of reducing statistical error). We determine the deposition rate as the mean among the rates inferred between every pair of thickness readouts (recording the thickness at $0.5 \mathrm{~Hz}$ ). In fact, measurements across the aperture correspond to the convolution of the response function with a $1 \mathrm{~mm}$ wide step function. As the response function should be Gaussian, we recursively adjust the amplitude and standard deviation for a Gaussian function until the convolutions of the resulting function with a $1 \mathrm{~mm}$ wide step function centered at the locations of a pair of data points agree with the corresponding measurements to within $1 \%$. We finally extract the response function by normalizing the resulting fit.

After measuring the response function in the pumping cross, we positioned the thickness monitor just behind the wire detector beyond the magnets. With the source (G3) at its optimal position, we measured deposition rates at several locations along the collection plane while operating the source at both $650{ }^{\circ} \mathrm{C}$ and $700{ }^{\circ} \mathrm{C}$. We displaced the sensor by fixed amounts corresponding to fractional turns of the handwheel on the translation assembly. We confirmed the relative position of the sensor at every measurement by using calipers to measure the distance between the chamber wall and one of the translating plates on the assembly. As shown in Fig. 4.8(A), we applied a constant offset to the relative positions of both sets of deposition rates in order to match the locations of the peak measurements to the maximum of a wire trace taken with the 

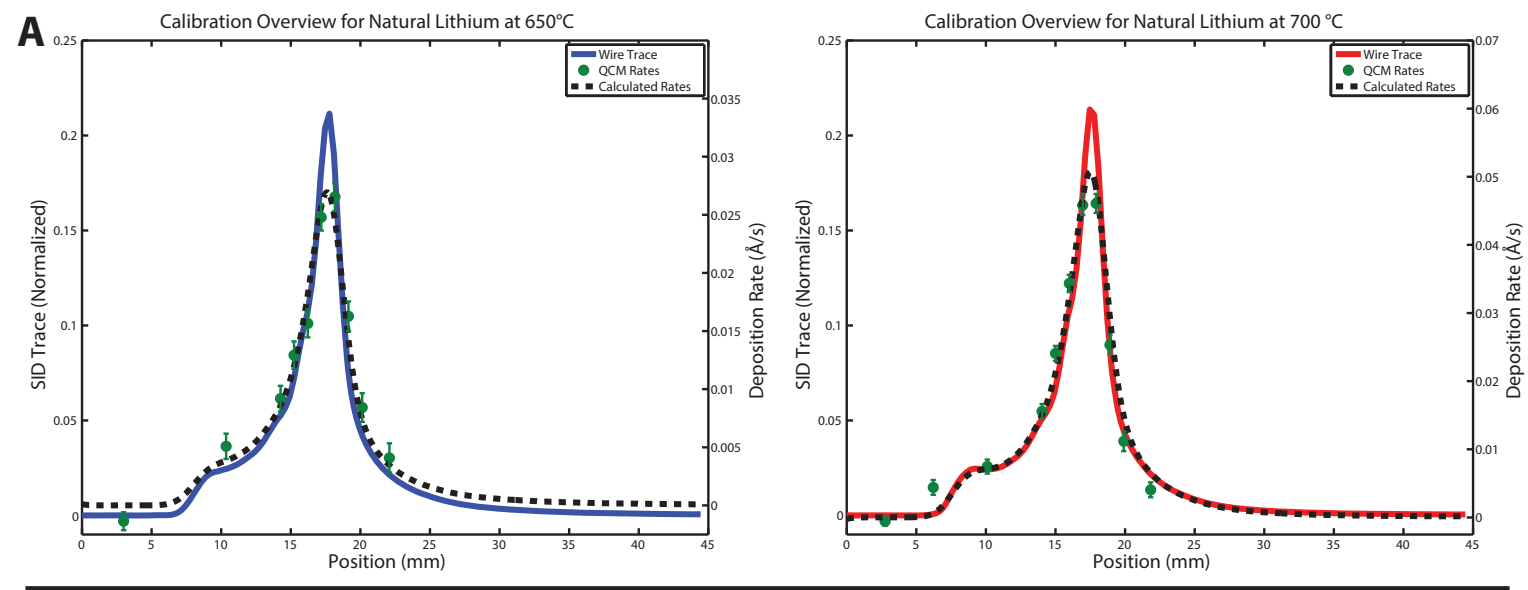

B

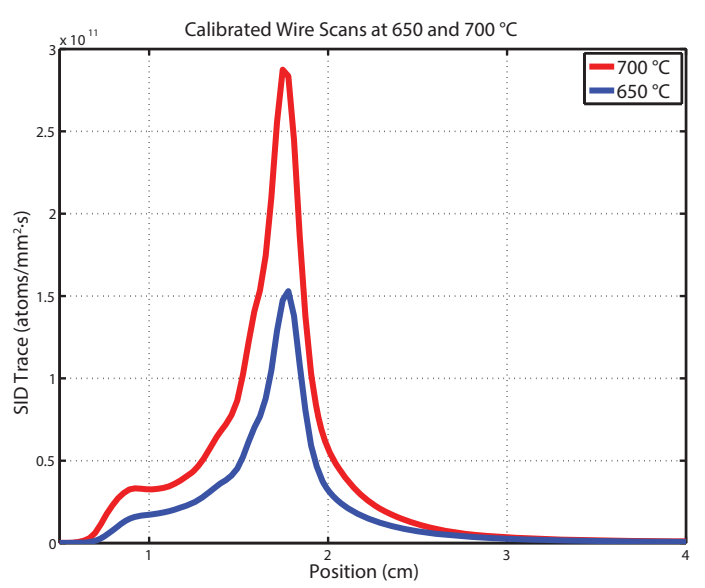

Figure 4.8: Summary of throughput measurements using natural lithium. (A) Using the measured response function, we obtain calibrations for a wire trace (blue/red) that reproduce thickness monitor measurements (green points show measurements and the dashed black lines shows calculations) upon taking convolutions with the response function accordingly. (B) Applying the calibrations to the wire trace, we obtain throughputs per s per mm of height at 650 and $700{ }^{\circ} \mathrm{C}$. 
source at $650{ }^{\circ} \mathrm{C}$.

For a given deposition rate $F_{Q C M}^{\prime}$ we then extract a calibration factor $\alpha^{\prime}$ for the wire trace that returns the deposition rate upon calculating the convolution of the portion of the trace overlapping the sensor position (centered at $x_{0}$ ) with the response function $R(x)$ that we previously determined. That is, we assume that we can write the flux measured by the thickness monitor at a given position as

$$
F_{Q C M}^{\prime}=\int_{x_{0}-r}^{x_{0}+r} \alpha^{\prime} n^{\prime}(x) R(x) d x
$$

where $r$ denotes the sensor radius and $n^{\prime}(x)$ is the normalized wire trace. Multiplying $F_{Q C M}^{\prime}$ by the atomic number density for lithium we can solve (4.1) for the calibration factor $\alpha$ of interest (with units given by atoms per s per $\mathrm{mm}^{2}$ ) as

$$
\alpha=\left(\frac{\rho_{\mathrm{Li}}}{m_{\mathrm{Li}}} F_{Q C M}^{\prime}\right) /\left(\int_{x_{0}-r}^{x_{0}+r} n^{\prime}(x) R(x) d x\right)
$$

where $\rho_{\mathrm{Li}}$ and $m_{\mathrm{Li}}$ denote the mass density and mass of lithium, respectively. Repeating this calculation for a set of thickness monitor measurements corresponding to the largest deposition rates, we obtain an effective calibration factor as the weighted average of the individual calibration factors (weighting by the relevant deposition rates). With this calibration, we observe agreement between almost all measured deposition rates and corresponding points on a curve generated across the entire collection plane using (4.1) as shown in Fig. 4.8(A).

For 650 and $700{ }^{\circ} \mathrm{C}$, these calibration factors are $(7.2 \pm 0.4) \times 10^{11}$ and $(1.34 \pm$ $0.02) \times 10^{12}$ atoms $/ \mathrm{mm}^{2} \cdot \mathrm{s}$ respectively. We estimate the error $\Delta \alpha$ in each calibration factor by first using the calibration for calculating deposition rates (using (4.1)) at the sensor positions where we made those measurements that contributed to the weighted average. We then solve for $\Delta \alpha$ by substituing the maximum deviation between these calculated rates and the associated measurements into (4.2). Using the calibrations for both source temperatures, we can scale the wire trace to obtain fluxes per second per $\mathrm{mm}^{2}$ beyond the magnets as shown in Fig. 4.8. By initially normalizing $n^{\prime}(x)$ and $R(x)$, the calibrations trivially give total fluxes per unit height $F_{Q C M}$ (obtained by integrating the wire traces $)$ of $(7.2 \pm 0.4) \times 10^{11}$ and $(1.34 \pm 0.02) \times 10^{12}$ atoms $/ \mathrm{mm} \cdot \mathrm{s}$ at 650 and 


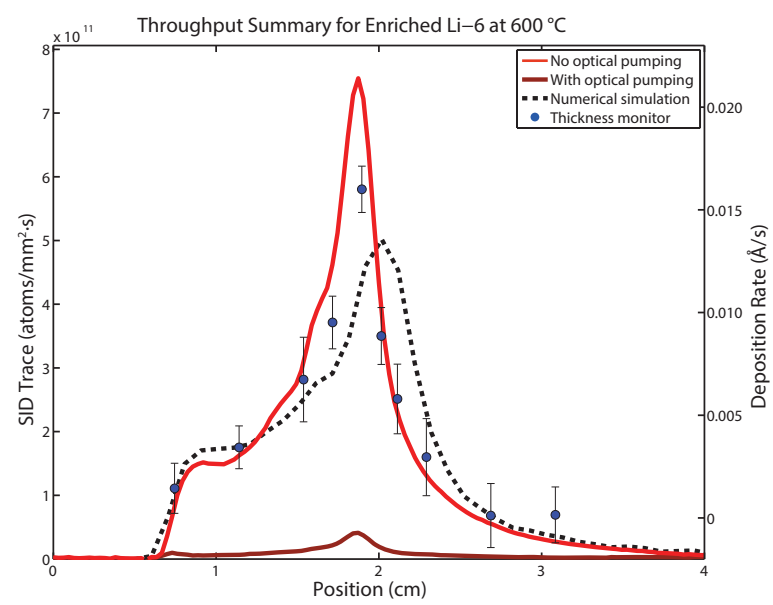

Figure 4.9: Summary of throughput measurements using enriched Li-6. We again use thickness monitor measurements (blue) for calibrating a wire scan (red). For comparison, we include a wire scan in the presence of optical pumping (dark red). We also include a profile resulting from a numerical simulation for a source operating at $800 \mathrm{~K}$ (dashed black line). We used the line-of-sight threshold evident in Fig. 4.2 to impose a horizontal offset in order to overlay the simulated and actual traces. We then scaled the amplitude of the simulated trace to obtain a reasonable match. We attribute the discrepancy between simulated and actual throughput to sensitivity to the exact arrangement of the magnet array. 


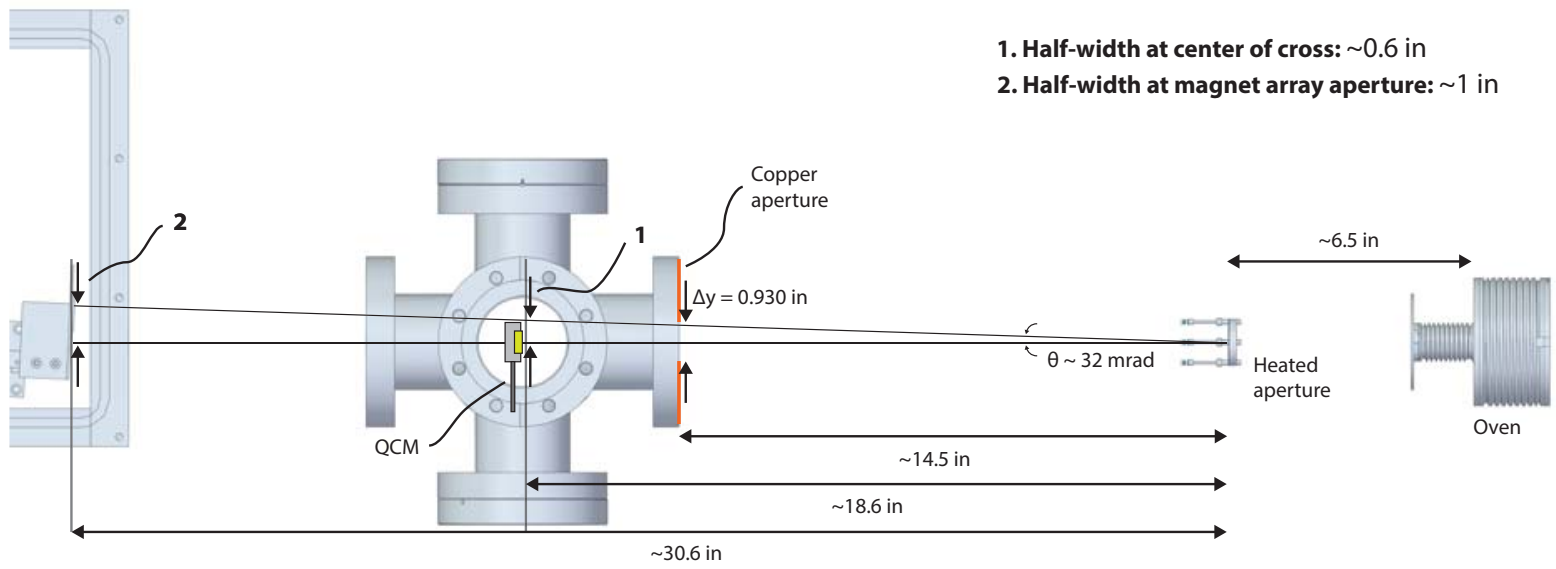

Figure 4.10: Overview of atomic beam geometry. For source G3, an aperture machined on the gasket at the cross entrance should restrict the beam widths at the center of the cross and the magnet aperture to 1.2 and 2 in respectively.

$700{ }^{\circ} \mathrm{C}$ respectively. These numbers should be weighted by the relative abundance of the isotope of interest in order to obtain the flux for that isotope. Using enriched Li-6 with the source $(\mathrm{G} 2)$ at $600{ }^{\circ} \mathrm{C}$, as shown in Fig. 4.9, we can similarly estimate the throughput to be $(5.4 \pm 0.7) \times 10^{11}$ atoms per s per mm of collection plane height ${ }^{2}$.

To estimate the efficiency of the guide at the two source temperatures considered above, we first determine the flux incident on the magnet aperture. We infer this flux by measuring deposition rates with the thickness monitor in the center of the pumping cross, then scaling this throughput based on the apparatus geometry. For this procedure to yield a valid estimate for flux incident on the magnets, the gradient in flux across the cross should be consistent with the apparatus geometry. Fig. 4.10 predicts the extent of the atomic beam at the cross center and magnet aperture based on the beam line geometry. The 0.930 in aperture machined onto a gasket at the cross entry should limit the beam widths at the cross center and magnet aperture to $\sim 1.2$ and $\sim 2$ in respectively ${ }^{3}$. Upon disassembling the apparatus, we confirmed that the width for the

\footnotetext{
${ }^{2}$ In this case, the isotope-specific throughput must be weighted by the relative abundances given by the enriched Li-6 in the source.

${ }^{3}$ Fig. 4.10 shows the source-to-aperture and source-to-cross distances for G3 while Fig. 4.11 gives measurements using G2. The distance between the heated aperture and the cross is the same in both cases, and the source-to-aperture distances are short enough for both sources that the aperture on the gasket at the cross entrance should be the feature defining the beam width.
} 


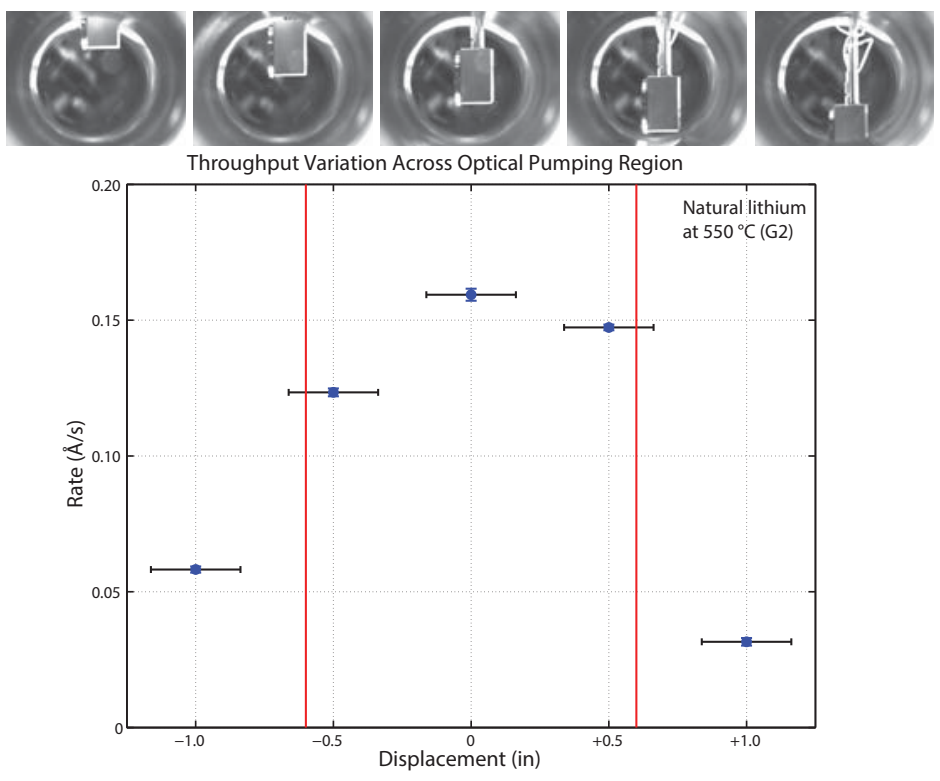

Figure 4.11: Translating thickness monitor across optical pumping region. We use the CCD to first calibrate the sensor width in pixels. We then retract the sensor from the cross and measure deposition rates (blue) as we advance the sensor by half-widths. Horizontal error bars show the extent $(8.25 \mathrm{~mm})$ of the sensor itself, and the red lines show the predicted width of the beam based on Fig. 4.10.

lithium that deposited onto the magnet aperture was almost exactly 2 in. Fig. 4.12 shows a photograph of lithium that had built up on the magnets over the course of this work.

Fig. 4.11 shows flux measurements obtained by translating the thickness monitor across the cross. Using the CCD, we first positioned the sensor as close as possible to the center of the cross. We then calibrated the width of the sensor body ( $\sim 1$ in) as a number of pixels, receded the sensor by one width, then made measurements by advancing the sensor by half-widths across the cross. Horizontal error bars denote the extent of the sensor itself and the red lines show the predicted extent for the atomic beam. Most importantly, the deposition rate is reduced upon translating away from the center of the cross. At the closest sensor positions to the center, we observe evidence of the aperture obstructing the incoming beam as the deposition rates drop slightly but remain within $75 \%$ of the peak at the center.

The strange behavior on the outermost measurements (notably the apparent 
asymmetry and the non-zero values) could be the consequence of one of several factors. For instance, the aperture might be offset from the beam line axis (as a consequence of machining tolerances or softening upon heating of the titanium standoffs supporting the aperture), thus producing asymmetric throughput across the pumping region ${ }^{4}$. Upon disassembling the apparatus, we observed that lithium had not condensed exactly symmetrically about the apertures machined onto various gaskets leading up to the cross, with the lithium on the closest aperture (protecting the gate valve) most notably appearing to be offset. The apparent beam diameter on the aperture at the cross, however, was roughly 1.25 in which is close to the expected width based on the reservoir opening and source-to-aperture distance ${ }^{5}$. Moreover, while disassembling we measured the beam diameter on the magnet aperture to be very close to 2 in (predicted by Fig. 4.10), with the aperture itself entirely contained within the beam.

After confirming reasonable variation in flux across the cross, we centered the sensor in the cross and measured deposition rates as a function of source temperature. Because the temperature-dependence of the flux is (expectedly) not perfectly reproducible, we repeated these measurements for all temperature-sensitive measurements beyond the magnets (including the measurements described above and fluorescence measurements described later). Fig. 4.13(A) shows two sets of measurements with enriched Li-6 in the source (G2) taken just prior to recording the flux data shown in Fig. 4.9. We observe agreement in measurements between the two dates up to the highest source temperature. We average the two points at $600{ }^{\circ} \mathrm{C}$ in order to determine the flux incident on the magnet aperture (for extracting the guide efficiency) ${ }^{6}$.

Fig. 4.13(B) shows two sets of data with natural lithium in the source (G3). We

\footnotetext{
${ }^{4}$ Equivalently, the nozzle might not be co-axial with the remaining beam line. Maintaining square joints during welding can be challenging.

${ }^{5}$ The outermost measurements in Fig. 4.11 might also be an artifact of the sensor itself. We sometimes observed a larger-than-expected deposition rate (particularly when measuring the sensor response function) under certain conditions upon immediately following a high-rate measurement. For this reason, we typically tried to make measurements from positions giving lower deposition rates to positions yielding higher rates.

${ }^{6}$ The discrepancy between these points could be a consequence of several factors. For instance, we took the first set of data just after re-loading and degassing the oven. Contaminants in the source might have contributed more heavily when taking the first set of measurements.
} 


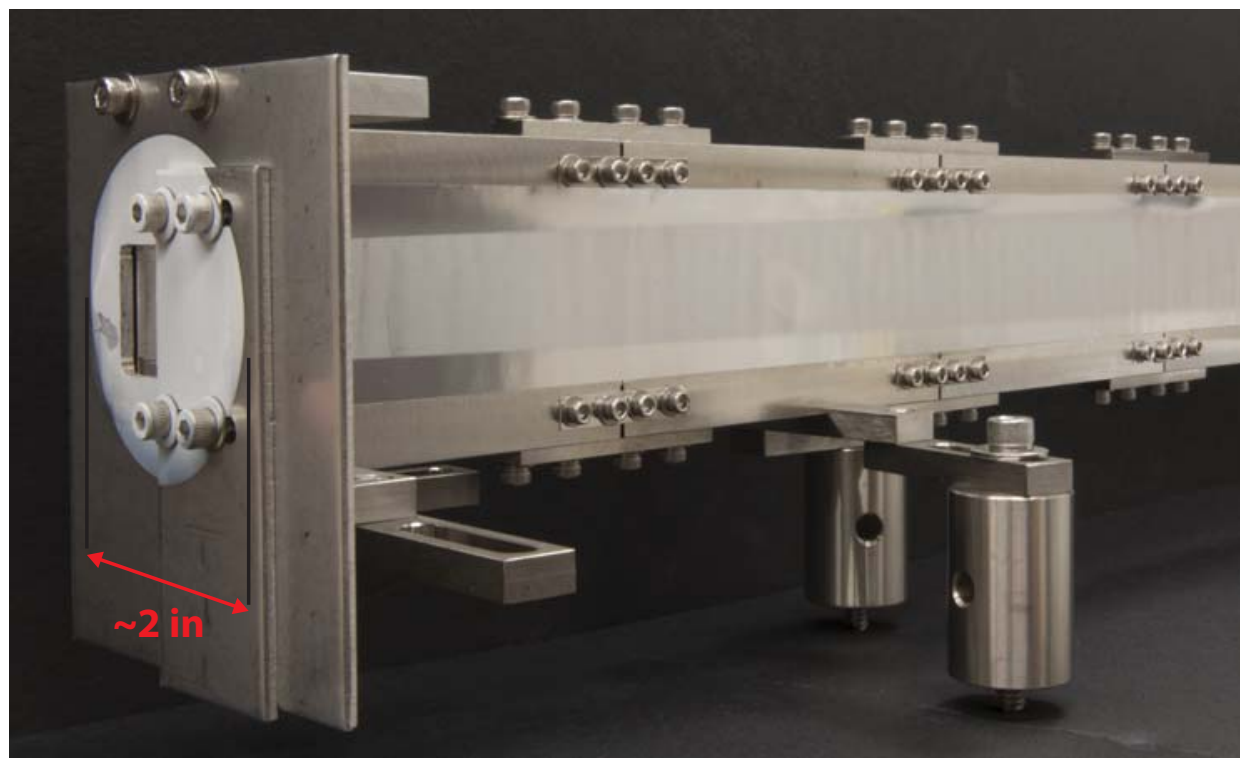

Figure 4.12: Lithium coating on magnet aperture (after removing magnets from chamber). The width of the coating on the magnet aperture was almost exactly the width that we had anticipated based on the apparatus geometry.

took one set immediately following the measurements shown in Fig. 4.24, and the other set just prior to the measurements shown in Fig. 4.8. We expected larger discrepancies here because we used different copies of the same source for the two data sets. Because of small variations (such as the exact positioning of thermocouples) between the ovens, a given nominal temperature for the ovens might have corresponded to slightly different actual temperatures between them. Again, contaminants (notably lithium compounds) could have contributed more heavily to the later data set. We had no means of measuring the cleanliness of the oven on the apparatus other than monitoring partial pressures of signatures of contamination (like hydrogen) on the RGA beyond the magnets.

Combining these measurements in the cross with those outlined by Figs. 4.8 and 4.9, we readily estimate the guide efficiency. With the solid angle angle subtended by a patch of flux to the source increasing along the beam direction (with trajectories close to the center-line of the beam axis propagating radially outward), the flux per unit area lessens accordingly. In particular, we can relate the measured flux in the cross $F_{\text {cross }}^{\prime}$ to 

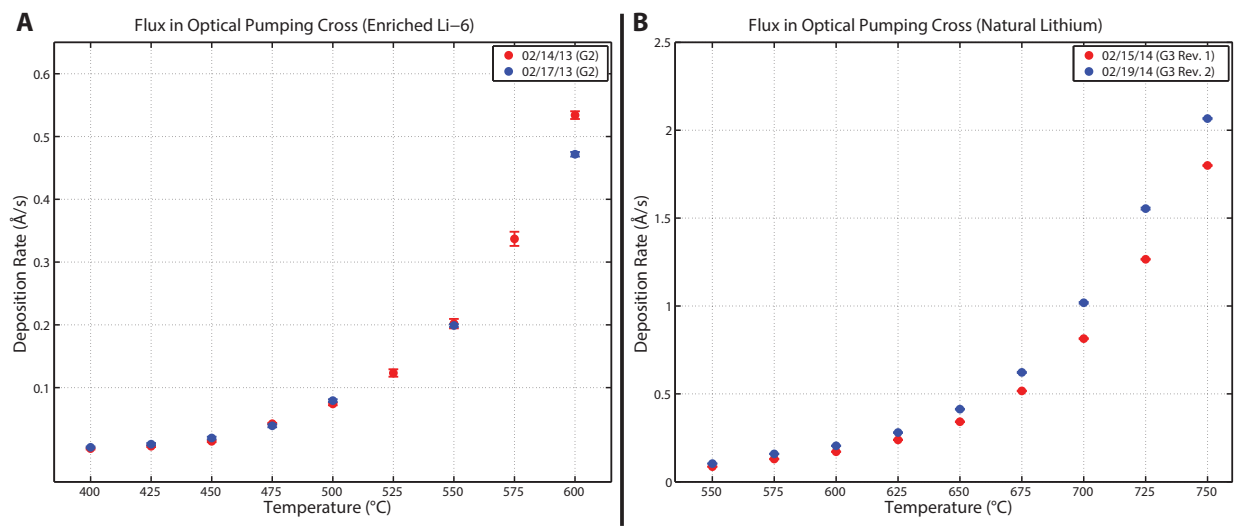

Figure 4.13: Various measurements of flux in optical pumping region as function of source temperature. (A) Two sets of measurements using G2 that we took just prior to the measurements outlined in Fig. 4.9. (B) Two sets of measurements using G3 giving the flux in the cross corresponding to data in Figs. 4.8 (blue) and 4.24 (red). Two copies of the same source generated these data.

the flux on the magnet entry $F_{\text {entry }}^{\prime}$ according to the apparatus geometry by

$$
F_{\text {entry }}^{\prime}=\left(d_{\text {cross }} / d_{\text {magnets }}\right)^{2} \times F_{\text {cross }}^{\prime}
$$

where $d_{\text {cross }}$ and $d_{\text {magnets }}$ are the source-to-cross and source-to-aperture distances as shown in Fig. 4.14(A) ${ }^{7}$. Multiplying the resulting flux by the magnet aperture width $w$, we obtain the flux per unit height $F_{\text {entry }}$ at the magnet entry.

As shown in 4.14(B), the flux into angular height $\theta$ at the magnet entrance is given trivially by

$$
h^{\prime} F_{\text {entry }}=\left(d_{\text {magnets }} \tan \theta\right) F_{\text {entry }} .
$$

For the same angular height, the flux beyond the magnets is given by

$$
h F_{Q C M}=\left(d_{Q C M} \tan \theta\right) F_{Q C M} .
$$

Assuming that all flux within this angular height $h$ beyond the magnets originates in the corresponding height $h^{\prime}$ at the magnet entry, we can estimate the efficiency $\epsilon$ by taking

\footnotetext{
${ }^{7}$ This relationship is valid immediately around the center of the beam line where the gasket apertures do not interfere with the cosine-dependence of the angular distribution for trajectories originating at the heated aperture [86].
} 
the quotient of (4.4) and (4.5):

$$
\epsilon=\left(d_{Q C M} \times F_{Q C M}\right) /\left(d_{\text {magnets }} \times F_{\text {entry }}\right) .
$$

We thus conclude that the guide efficiencies when using natural lithium (with G3) at nominal source temperatures of 650 and $700{ }^{\circ} \mathrm{C}$ are given by $0.26 \pm 0.04$ and $0.21 \pm 0.03$ respectively. Likewise, the guide efficiency when using enriched Li-6 (with G2) at a nominal source temperature of $600{ }^{\circ} \mathrm{C}$ is $0.17 \pm 0.03^{8}$.

As outlined previously, these throughput measurements can be geometrically scaled based on the number and size of the guides. As the magnet array is twodimensional, scaling the throughput without sacrificing purity proceeds by extending the height of the arrays and arranging additional arrays about the source. Per vertical meter of guide entrance, the measured throughputs above for natural lithium scale linearly to $0.8 \pm 0.1$ and $1.6 \pm 0.2$ grams per year of continuous operation. Thus, 50 guides each $3 \mathrm{~m}$ tall arranged about a source operated nominally at $700{ }^{\circ} \mathrm{C}$ could produce in excess of 200 grams per year of enriched Li-7. Engineering guides to maximize the solid angle subtended by the collection plane to the source will enable even larger throughputs. On a commercial apparatus, the efficiency per guide gives an upper limit for overall efficiency as not all material evaporated from the source will reach a guide. By engineering the apparatus such that the guide entrances subtend a large fraction of the solid angle to the source, the overall efficiency should be a large fraction of the estimates given here. Using a larger source area will yield a comparable flux at lower source temperatures where the guide efficiency is better

\footnotetext{
${ }^{8}$ The lower efficiency in this case likely stems from the oven position being further away from lineof-sight.
} 


\section{A (TOP DOWN)}
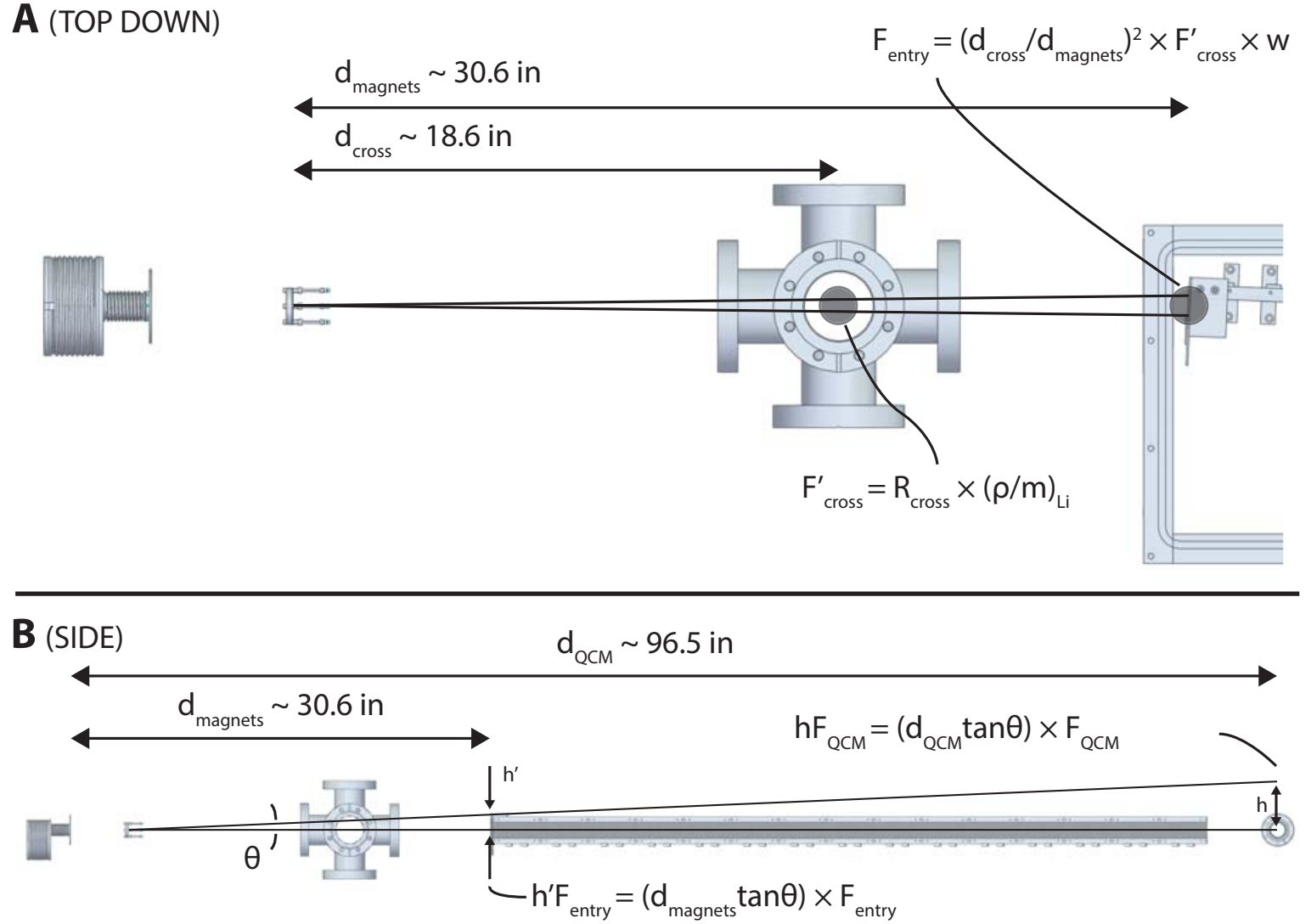

Figure 4.14: Overview of procedure for estimating guide efficiency. (A) As the beam expands between the cross and the magnet aperture, the flux per unit area decreases in accordance with the apparatus geometry. Multiplying the measured deposition rate (in units of thickness per second) by the atomic number density for lithium gives a flux per unit area. (B) Measurements give the flux per unit height at the magnet aperture and beyond the magnets. Taking the quotients of these fluxes (multiplied by the same angular height), we obtain estimates for the guide efficiency. 

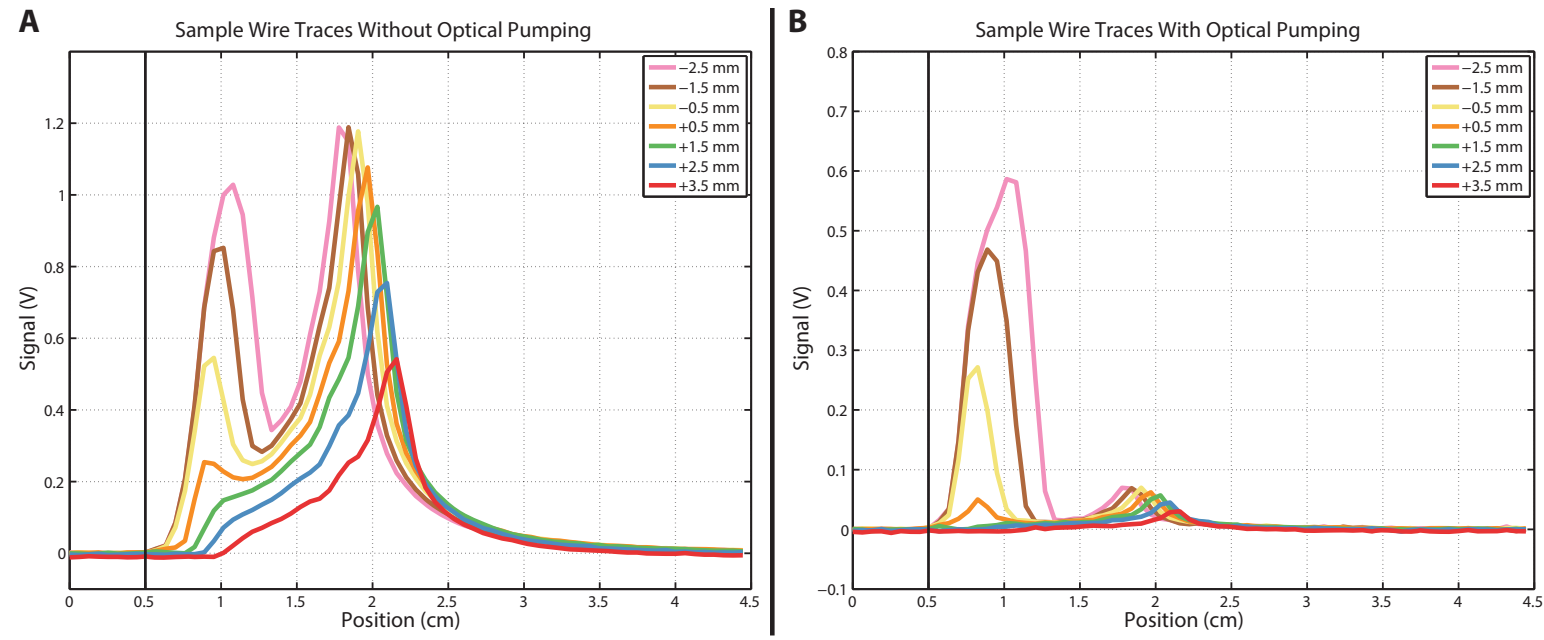

Figure 4.15: Wire scan traces with (B) and without (A) optical pumping using enriched Li-6. We observe substantial throughput suppression across the width corresponding to atoms that reflect from the magnets. Radiation pressure reduces the flux corresponding to atoms having line-of-sight to the collection plane.

\subsection{Enrichment}

We first visualize Li-6 suppression using the wire detector. Fig. 4.15 compares background-subtracted wire scans (using enriched Li-6 in the source) as a function of oven position both in the absence and presence of optical pumping. We first observe substantial suppression of the throughput among the contribution to the collection plane corresponding to trajectories that reflect from the magnets. As expected, the traces granting some line-of-sight between the source and collection plane show far less depletion over the region of the throughput corresponding to those trajectories having line-of-sight. We attribute the mild suppression of this throughput primarily to radiation pressure that offsets the angular distribution for Li-6 atoms incident on the magnet array.

As shown in Fig. 4.16 we can further investigate Li-6 depletion by taking the quotient of the wire signals with and without optical pumping across the collection plane. Across the region of the plane corresponding to trajectories that reflect from the magnets, we observe nearly uniform suppression to roughly $5 \%$ of the throughput in the absence of pumping. This fraction is consistent with the nominal enrichment 

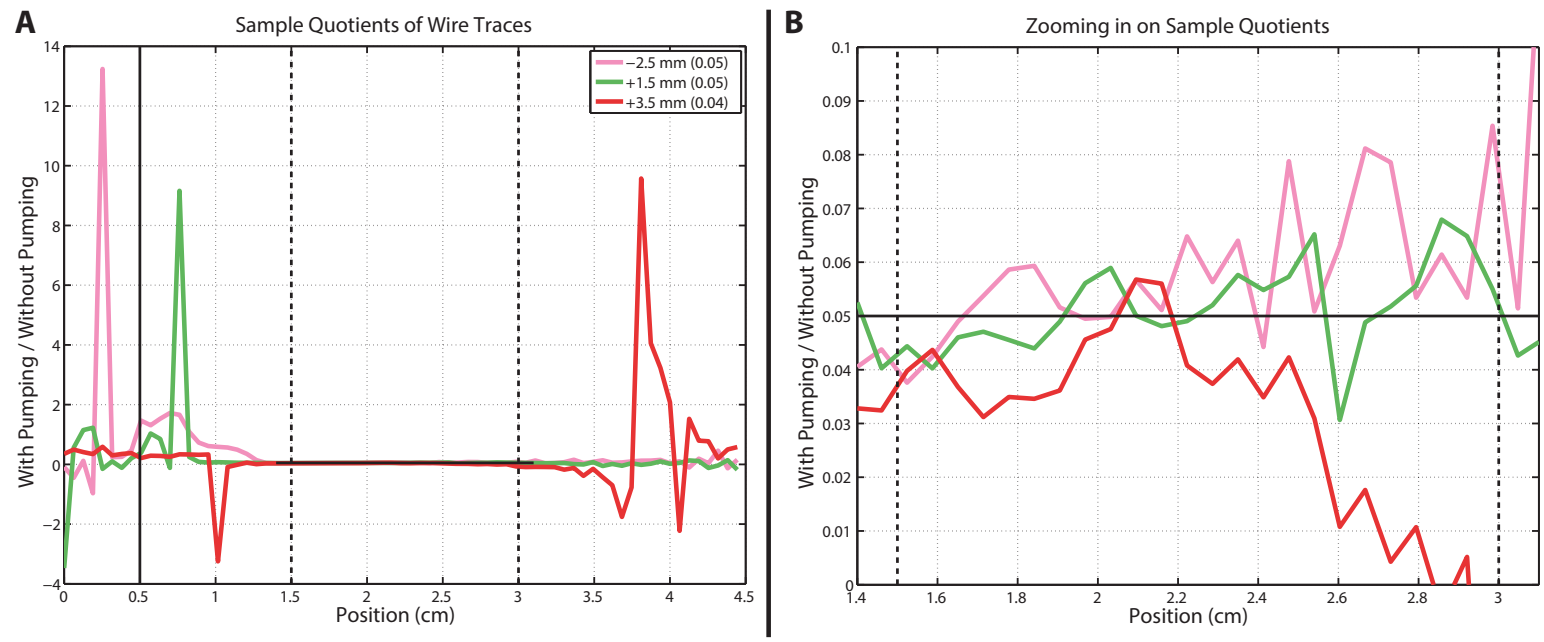

Figure 4.16: Quotients of traces with and without optical pumping (using enriched Li6). (A) We observe uniform depletion across most of the collection plane. The quotient exceeds one immediately adjacent to the magnet shadow for quotient at $-2.5 \mathrm{~mm}$ likely because of radiation pressure offsetting the throughput. (B) The quotients reasonably agree wtih the nominal isotopic fraction of $\mathrm{Li}-7$ in the source.

of Li-6 within the source (95\%). Without precisely knowing the relative abundances of Li-6 and Li-7 in the throughput, we cannot quantitatively infer a depletion factor using these quotients ${ }^{9}$. Nonetheless, the quotients show promising characteristics that are consistent with substantial Li-6 suppression. Adjacent to the magnet shadow, this quotient is greater than one over an extended width for the oven position closest to absolute line-of-sight. As the line-of-sight contribution lessens in the presence of optical pumping, this region where the quotient exceeds unity provides further evidence that radiation pressure has offset the angular distribution incident on the magnet array.

We can perform similar analysis with natural lithium in the source as shown in Fig. 4.17. Deciphering features of Li-6 suppression in this case is even more challenging due to the substantially higher background (corresponding to Li-7). Again, however, we observe close-to-uniform reduction in signal across the width of the plane corresponding to trajectories that reflect from the magnets. The quotients of traces with and without

\footnotetext{
${ }^{9}$ Uncertainty in the isotopic fraction of Li- 7 of just $1 \%$ around the nominal value can yield large uncertainties when extracting a value for Li- 6 suppression (with this ultimate uncertainty worsening for better Li-6 suppression).
} 

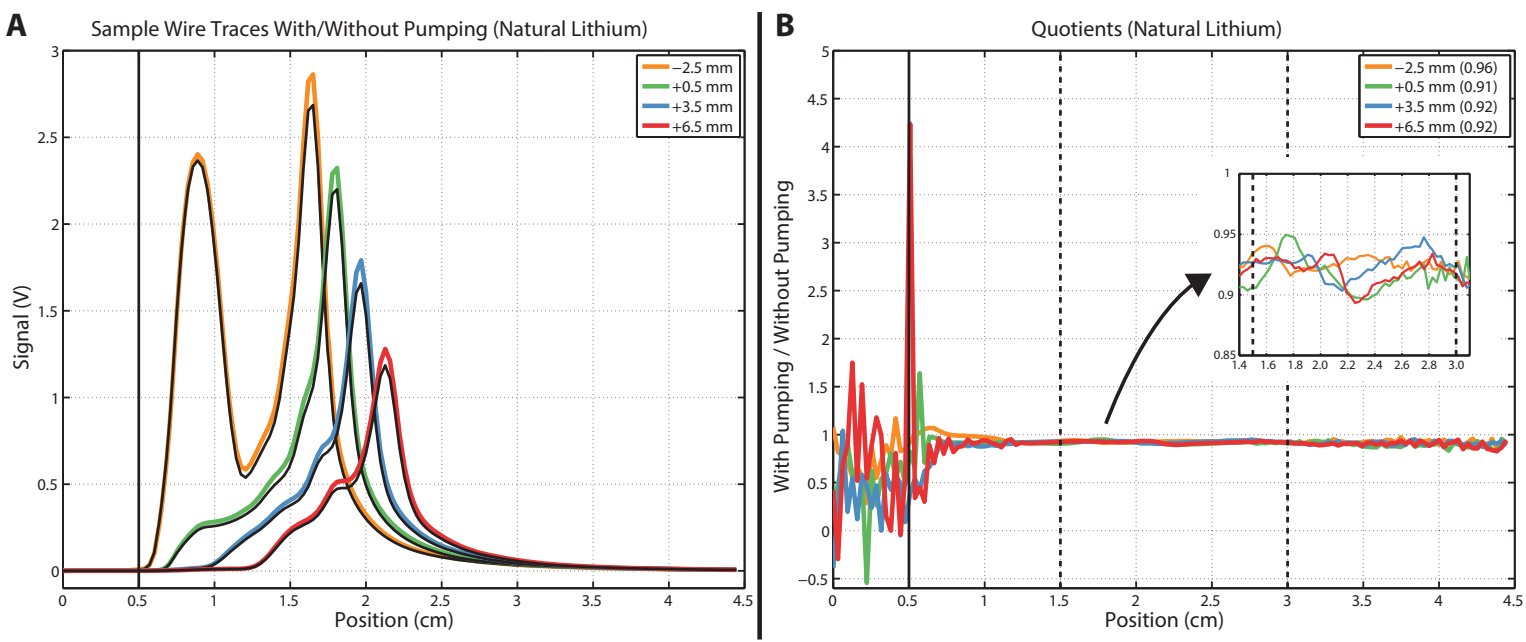

Figure 4.17: Wire scan traces (and corresponding quotients) with and without optical pumping using natural lithium. (A) All traces in the presence of optical pumping show lower flux than corresponding measurements in absence of pumping. (B) Again, quotients agree reasonably with the nominal isotopic fraction of $\mathrm{Li}-7$ in the source.

pumping are again consistent with the nominal Li-7 fraction in the beam (92.5\%) over the region of interest. This quotient again exceeds one in the region immediately adjacent to the guide shadow for an oven position granting line-of-sight.

Fig. 4.18 compares wire traces (again using natural lithium with the source at a position granting line-of-sight) when tuning the laser to both the $\mathrm{F}=1 / 2$ and $\mathrm{F}=$ $3 / 2$ ground states. As already shown in Fig. 4.17, pumping into the $\mathrm{F}=1 / 2$ state (with the laser tuned to the $\mathrm{F}=3 / 2$ state) uniformly reduces throughput within the width of the dashed rectangle drawn on the figure. When tuning the laser to the $\mathrm{F}=$ $1 / 2$ state, however, the peak signal increases as we enhance the number of Li-6 atoms in the partially low-field seeking $\mathrm{F}=3 / 2$ state. Moreover, optical pumping in this case has laterally offset the distribution slightly as radiation pressure adjusts the incidence angles for Li-6 atoms at the magnet entrance ${ }^{10}$.

Fig. 4.19 gives mass spectra between 4 and 10 amu measured using the electron

\footnotetext{
${ }^{10}$ Part of this offset might be due to slight hysteresis in the stepper motor upon reverting the wire detector back to its initial position upon completing a scan. We usually manually check the starting position (using a position readout on the linear actuator) prior to running, but occasionally will miss a slight offset in starting position.
} 


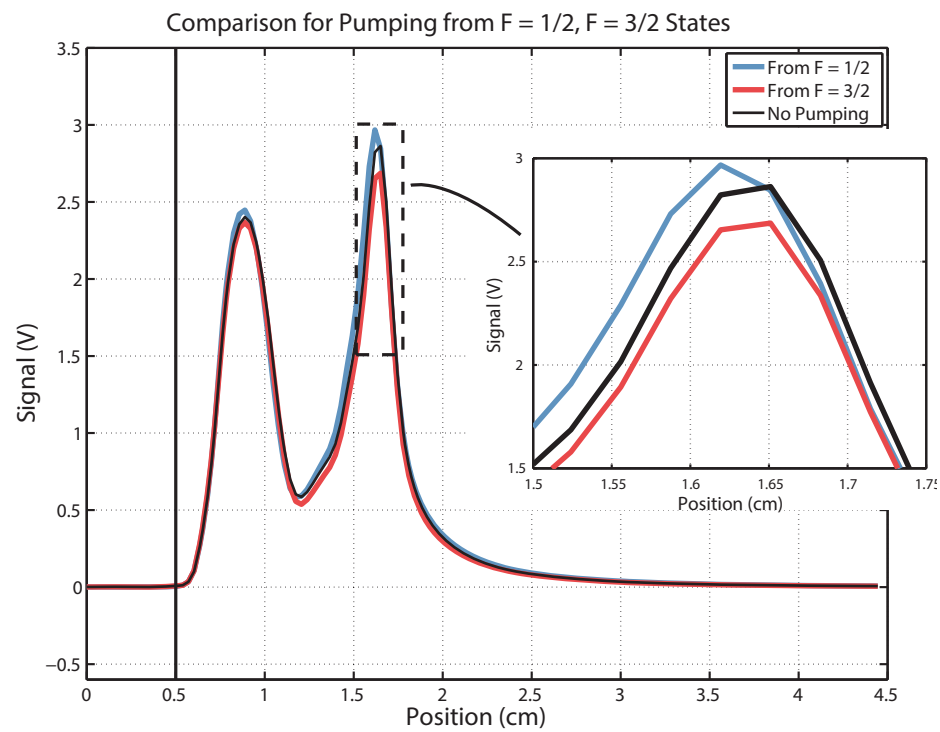

Figure 4.18: Comparison of wire scan traces when turning the optical pumping laser to the $\mathrm{F}=3 / 2$ and $\mathrm{F}=1 / 2$ ground states. Throughput uniformly worsens when pumping atoms out of the $\mathrm{F}=3 / 2$ state (red) in comparison to throughput measured without optical pumping (black). In contrast, we enhance and slightly offset throughput when pumping out of the $\mathrm{F}=1 / 2$ state (blue).

multiplier on the RGA with enriched Li-6 in the source (G1). We operated the electron multiplier at its peak operating voltage (limited likely by gain degradation as we discussed earlier), corresponding to a gain in excess of 150,000. We scan the mass at the slowest allowable rate in order to maximize averaging time at a given mass, and step across the spectrum by $0.1 \mathrm{amu}$ increments ${ }^{11}$. At nominal source temperatures of 550 and $600{ }^{\circ} \mathrm{C}$, we recorded (in rapid succession) a collection of spectra with optical pumping, without optical pumping, and with no atoms. After averaging the spectra for each case (and inferring uncertainties at every data point as standard deviations of the mean), we then compared the background-subtracted signals with and without optical pumping at masses 6 and 7 .

For these measurements, we measured the laser power (prior to the beam shaping optics) to be close to $70 \mathrm{~mW}$. With a $100 \mu \mathrm{m}$ pinhole in front of the laser power meter

\footnotetext{
${ }^{11}$ For analog scans, we use software provided by SRS for operating the RGA. For single-mass measurements, we use a NI LabVIEW program that we developed for controlling the RGA.
} 

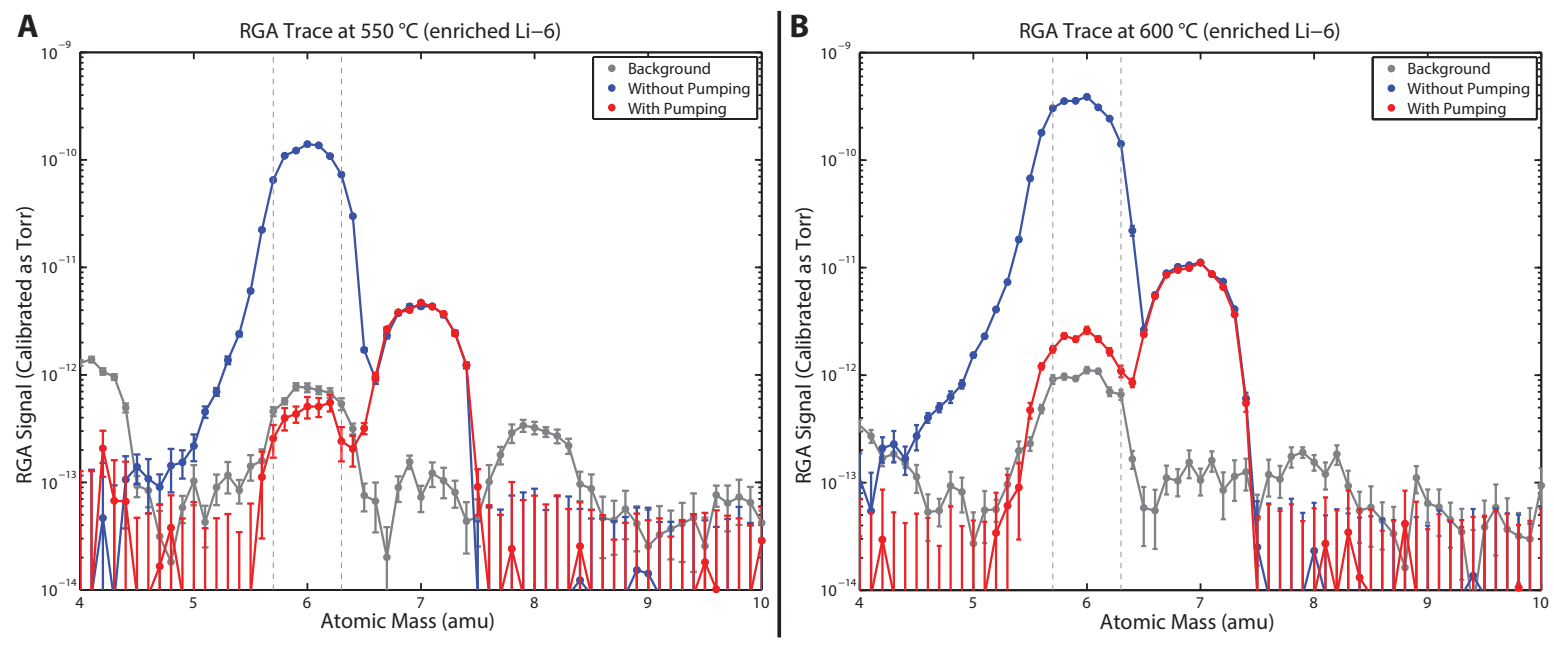

Figure 4.19: Background-subtracted RGA spectra in presence and absence of optical pumping (using enriched Li-6). (A) At $550{ }^{\circ} \mathrm{C}$, we reduce Li-6 throughput (at exactly 6 $\mathrm{amu}$ ) upon applying optical pumping (red) to $(3.6 \pm 0.8) \times 10^{-3}$ times its value without applying optical pumping (blue). The signal at 7 amu remains unchanged. (B) At 600 ${ }^{\circ} \mathrm{C}$, we suppress Li- 6 by a factor of $150 \pm 10$.

sensor, we estimated the peak intensity of the beam passing through the cross to be close to $17 \mathrm{~mW} / \mathrm{cm}^{2}$. We used linear polarization without using the Helmholtz pairs for defining a quantization axis, and we applied approximately $0.26 \mathrm{~W}$ onto the EOM for broadening the laser spectrum. On the resulting spectra, we first observe that the signal at mass 7 amu remains unaffected by the laser as desired. At exactly 6 amu, we suppress throughput upon applying optical pumping to $(3.6 \pm 0.8) \times 10^{-3}$ and $(6.7 \pm 0.5) \times 10^{-3}$ times its values in the absence of pumping at 550 and $600{ }^{\circ} \mathrm{C}$ respectively. That is, at these temperatures we suppress Li-6 throughput by factors of $280 \pm 60$ and $150 \pm 10$ respectively. When sampling a single mass, the RGA software performs a 0.6 amu wide scan about the mass of interest. Taking the mean depletion value for masses within this window around $6 \mathrm{amu}$ for the scans at 550 and $600{ }^{\circ} \mathrm{C}$, we alternatively obtain depletion factors given by $260 \pm 30\left((3.8 \pm 0.4) \times 10^{-3}\right)$ and $150 \pm 10\left((6.6 \pm 0.2) \times 10^{-3}\right)$ respectively ${ }^{12}$.

Fig. 4.20 shows Li-6 depletion - measured using the RGA with the source (G2)

\footnotetext{
${ }^{12} \mathrm{In}$ fact, during its procedure the RGA selects the largest ion current within the $0.6 \mathrm{amu}$ window (not computing an average).
} 


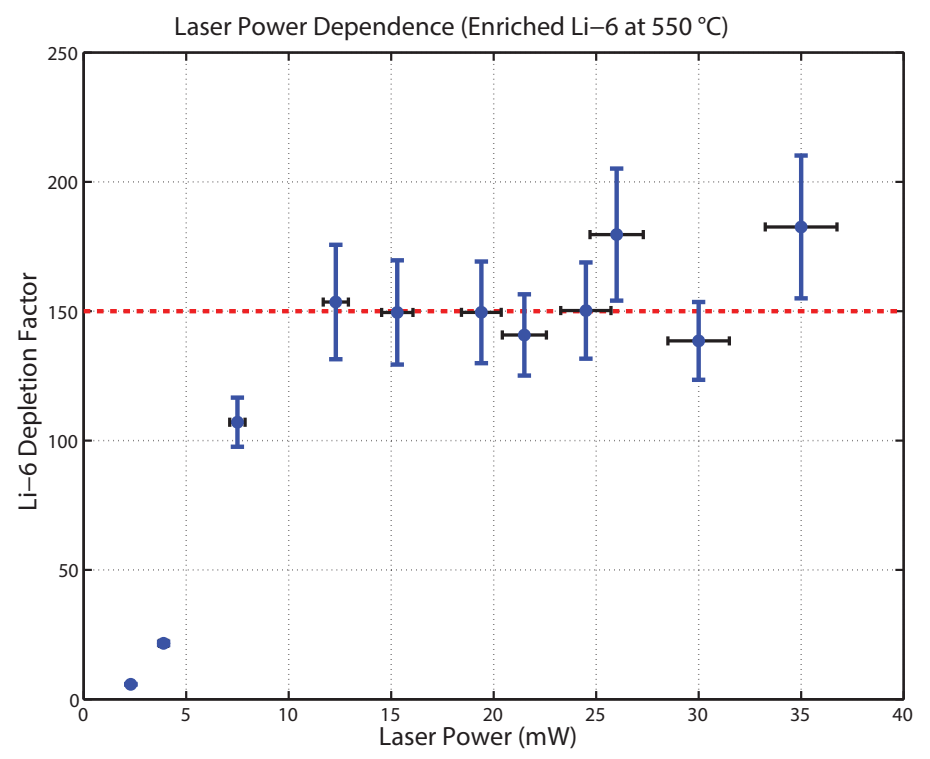

Figure 4.20: Dependence of Li-6 suppression on total laser power (using enriched Li-6). We measured laser powers by focusing light passing the cross onto a laser power sensor. Horizontal error bars reflect the $5 \%$ error in the calibration for an attenuator on the sensor.

operating at $550{ }^{\circ} \mathrm{C}$ - as a function of the power in the optical pumping beam ${ }^{13}$. We attenuated the laser power by installing combinations of neutral density filters in the optical beam line prior to the beam shaping optics. We measured power by removing the retroflecting mirror on the opposite side of the cross and focusing the light passing through the chamber onto the power meter. The maximum power $(\sim 35) \mathrm{mW}$ corresponds to a peak intensity of close to $12 \mathrm{~mW} / \mathrm{cm}^{2}$ (determined by scanning the power meter across the beam beyond the cross with a $100 \mu \mathrm{m}$ pinhole mounted in front of the sensor). We observe little change in Li-6 suppression until reducing the power below $10 \mathrm{~mW}$. Given the beam dimensions, $10 \mathrm{~mW}$ should correspond to an average intensity across the beam of roughly $2.5 \mathrm{~mW} / \mathrm{cm}^{2}$ (or less than half the intensity of the saturation intensity for the Li-6 D1 line). This data suggests that the rate for pumping atoms in to the $\mathrm{F}=1 / 2$ ground state is sufficient as long as the laser intensity remains close to saturation over the laser width.

\footnotetext{
${ }^{13}$ For these measurements we use custom software (built using NI LabVIEW) that samples single masses.
} 


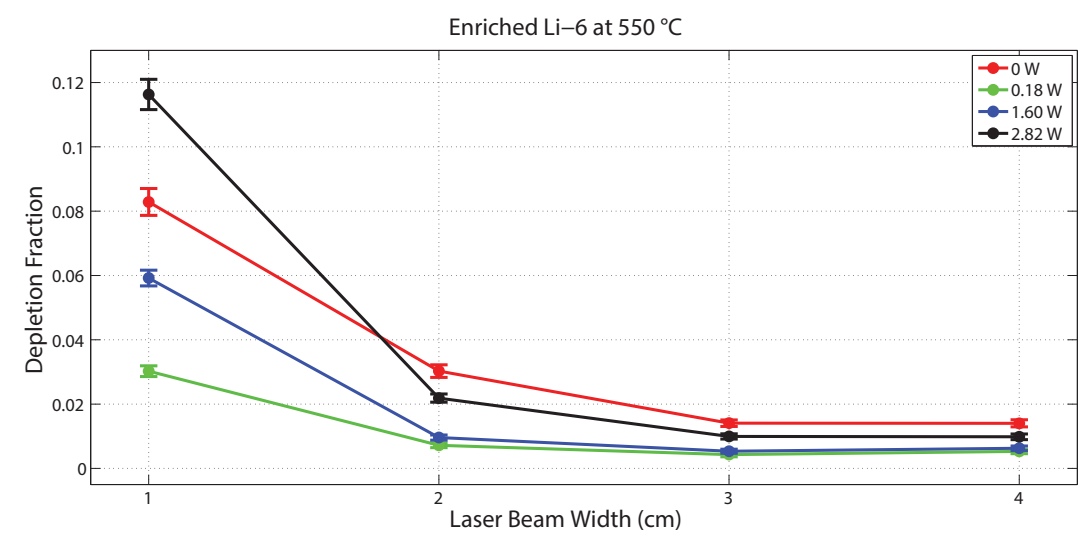

Figure 4.21: Dependence of Li-6 suppression on spectral broadening and laser beam width. Beam widths correspond to the width of a jury-rigged variable aperture directly in front of the pumping cross (determined using calipers). See Fig. 3.12 for frequency spectra corresponding to RF powers incident on the EOM.

Fig. 4.21 shows additional depletion measurements - again obtained using the RGA with the source (G1) operating at $550{ }^{\circ} \mathrm{C}$ - as we vary both the width of the laser beam (and thus interaction time) and power applied to the EOM. We set the beam width by adjusting the opening on the jury-rigged variable slit just in front of the viewport on the pumping cross. Interestingly, at the smallest width $(1 \mathrm{~cm})$ we observe better depletion without broadening the laser spectrum than upon applying maximal broadening. Upon opening the aperture to $2 \mathrm{~cm}$, we measure the worst depletion in the absence of any broadening. We thus hypothesize that the intensity per unit frequency is too far below saturation for certain frequencies of interest in the case of maximal broadening. At the smallest width, the interaction time is insufficient for the resulting pumping to be as effective as the case of no broadening despite (in principle) better overlap between the laser spectrum and the angular distribution incident on the magnets. Widening the slit to $2 \mathrm{~cm}$ and beyond allows for enough interaction time upon applying maximal broadening for more atoms to collect in the $\mathrm{F}=1 / 2$ state (in comparison to the case of no broadening).

For all beam widths, we observe best depletion when applying $0.18 \mathrm{~W}$ on the EOM (notably when comparing against the case of applying $1.60 \mathrm{~W}$ on the EOM). Among the RF powers that we considered, at $0.18 \mathrm{~W}$ we appear to optimally distribute laser 
power across those frequencies that comprise the angular distribution for trajectories that impinge on the magnet array. In principle, with sufficient laser power further broadening should only improve depletion (until the beam addresses all relevant trajectories). The EOM, however, provides a coarse mechanism for beam broadening as RF power on the EOM does not uniformly distribution power across frequencies. For instance, at certain $\mathrm{RF}$ powers (such as the half-wave voltage) power at certain frequencies will be completely suppressed. Thus, a particular RF power on the EOM might more efficiently address a given subset of the angular distribution than another RF power, and vice versa. The measurements in Fig. 4.21 indicate that more uniform broadening of the laser spectrum should enable better depletion.

We were unable to pursue meaningful depletion measurements on the RGA when using natural lithium. As shown in Fig. 4.22, the signal at 7 amu bleeds heavily into the signal at 6 amu. Default calibration of the quadrupole mass filter provides close to $1 \mathrm{amu}$ resolution across the entire range of the spectrometer. That is, the ion current at a given mass should fall to $10 \%$ of its peak value within \pm 0.5 amu of that mass. For natural lithium, however, $10 \%$ of the $7 \mathrm{amu}$ signal (presumably the ion current at $6.5 \mathrm{amu}$ ) will exceed the signal at $6 \mathrm{amu}$. Not surprisingly, Li-7 contributes a nonnegligible background at $6 \mathrm{amu}$ that worsens between 6 and 6.5 amu. As shown in Fig. 4.22(B), depletion improves between 6.3 and $5.7 \mathrm{amu}$. We attempted to correct for this background by enhancing the mass resolution at 6 and $7 \mathrm{amu}$. Improving the mass resolution, however, worsens the efficiency for ions to pass the mass filter onto the electron multiplier. As we were not able to obtain a suitable balance between mass resolution and signal, we ultimately pursued fluorescence detection for analyzing Li-6 suppression while using natural lithium. 

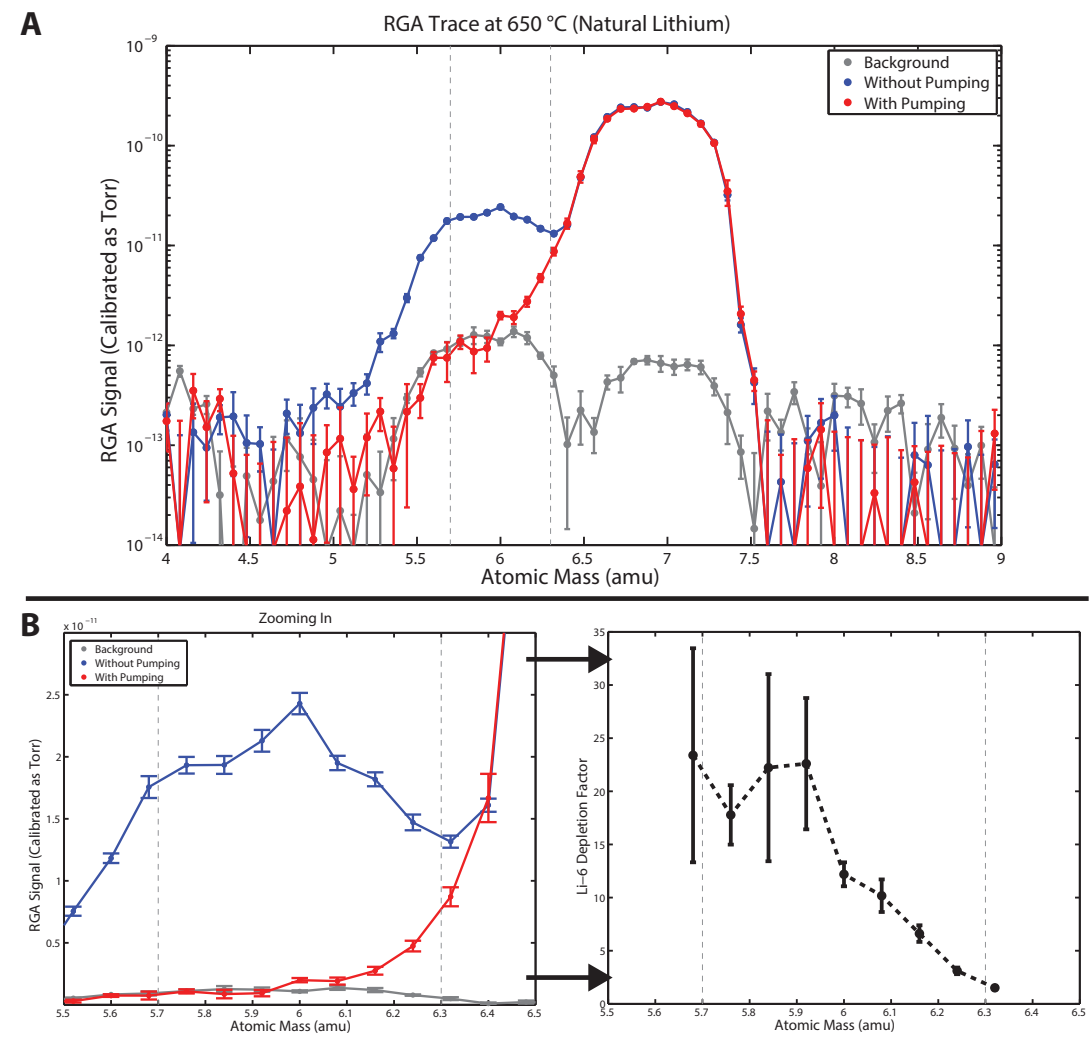

Figure 4.22: Overview of difficulty in using RGA for analysis of Li-6 suppression when using natural lithium. (A) Non-optimized mass spectrum showing 7 amu signal "bleeding" into 6 amu signal. (B) Zooming in on (A) and calculating depletion values at every point along the scan. 


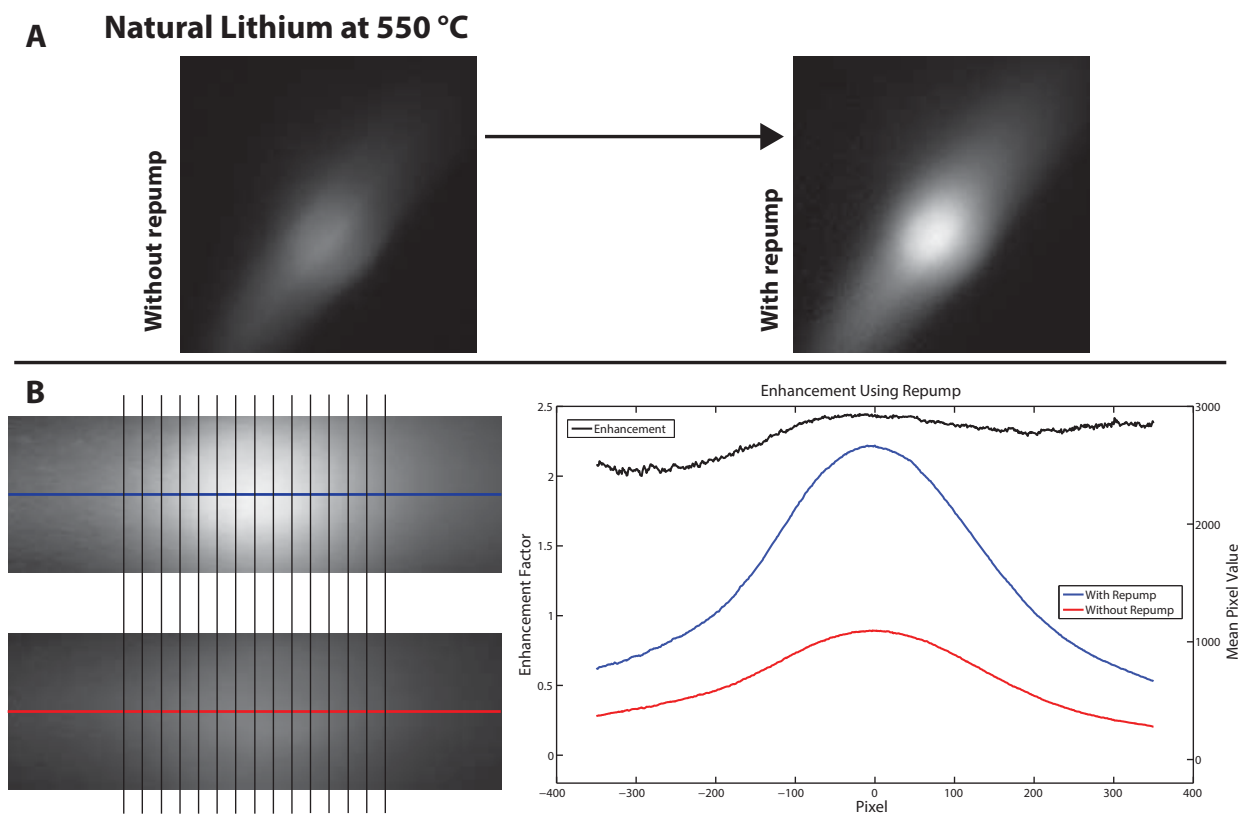

Figure 4.23: Enhancement of fluorescence signal in presence of repumping beam. (A) We position the CCD and imaging optics to roughly center the fluorescence on the sensor. Images taken with (right) and without (left) the repumping beam at constant exposure show drastic enhancement in fluorescence. (B) Comparing the enhancement across the observed fluorescence with (blue) and without (red) the repumping beam indicates enhancement by a factor of $\sim 2.25 X$ with peak-to-peak variation of $20 \%$.

To setup fluorescence detection, we first directed the fluorescence beam across the collection plane through a 1-1/3 in viewport on the chamber body onto an absorptive filter. After imaging the fluorescence close to the center of the CCD sensor, we adjusted the beam angle and tilt in order to maximize the total background-subtracted fluorescence. We wrote software (using NI LabVIEW) that dynamically returned the total intensity (corresponding to the sum of all pixel values) within a cropped area of the image (chosen to be centered around the peak signal). For all measurements, we operate the $\mathrm{CCD}$ at $-20{ }^{\circ} \mathrm{C}$ with the exposure between 1 and 10 s. Fig. 4.23 compares background-subtracted images of the fluorescence (without optical pumping prior to the magnets) in the absence and presence of the repumping beam. Integrating the pixel values over the entire sensor for both images indicates that the repumping beam provides a $\sim 2.25 \mathrm{X}$ enhancement in signal.

After cropping the original images accordingly, the percent different between 
the minimum and maximum enhancement at corresponding pixel locations along the incident laser is roughly $20 \%$. The apparent gradient in the enhancement along the laser direction might be an artifact of the procedure for image processing. As discussed later, we apply a script using an image editor (Adobe Photoshop CS6) for rotating and cropping images to center fluorescence in the frame. Running independent scripts for images captured using the repump and for those taken without the repump, the fluorescence maxima might be slightly offset. The enhancement gradient might also be a consequence of either slight misalignment between the main and repumping beams or miniscule detuning of the repumping beam away from the $\mathrm{F}=1 / 2$ ground state.

Fig. 4.24 shows both Li-6 suppression over a range of source temperatures and deposition rates measured in the pumping region just prior to making the depletion measurements. To extract these depletion factors, we followed a similar procedure to that described above for measuring the effect of the repumping beam. Using our data acquisition software, we first defined a cropped area for the fluorescence image that both centered and contained the brightest area of the fluorescence. We chose this area in the absence of optical pumping as the depletion was sufficient to make any fluorescence unnoticeable by eye in the presence of optical pumping (even at very long exposure times in excess of $10 \mathrm{~s}$ ).

At every temperature, we then captured a number of images (both cropped areas and original frames) without optical pumping, with optical pumping, and with no atoms. In particular, for the data shown in Fig. 4.24 we captured 60 images per measurement (20 for each of the three conditions). For all measurements we used $5 \mathrm{~s}$ exposures (except at the two highest temperatures where we reduced the exposure to $4 \mathrm{~s}$ ). For a given data point, we first calculated the sum over all pixel values for each cropped image of a particular set, then determined the mean of these sums (taking the standard deviation of this mean as a statistical error bar). We ultimately extracted the depletion factor as:

$$
\text { Depletion Factor }=\frac{\overline{P_{\text {without }}}-\overline{P_{\text {background }}}}{\overline{P_{\text {with }}}-\overline{P_{\text {background }}}}
$$

where $\overline{P_{\text {without }}}, \overline{P_{\text {with }}}$, and $\overline{P_{\text {background }}}$ denote the averages of the relevant sums over 

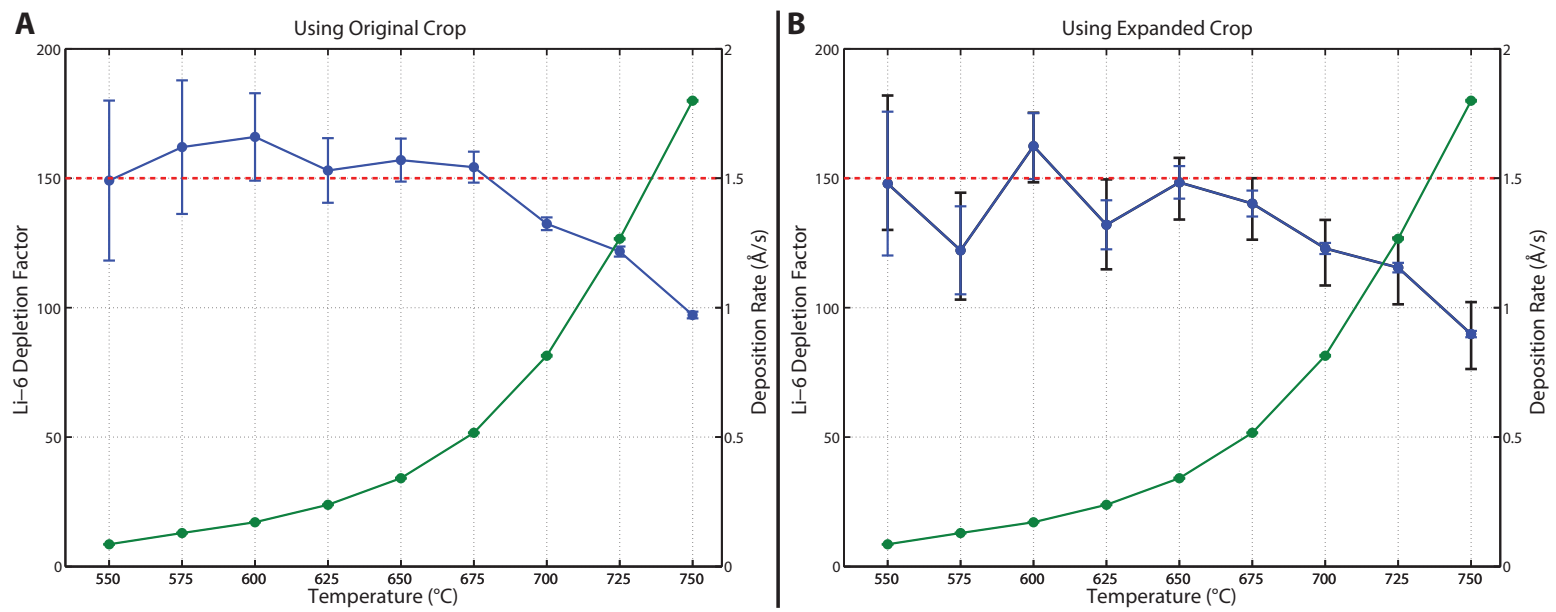

Figure 4.24: Depletion dependence on source temperature (for natural lithium). (A) We initially extracted depletion values by comparing integrated pixel values of backgroundsubtracted fluorescence measured with and without optical pumping within a common cropped region of the original images. (B) We alternatively extracted depletion values by using a script for independently processing images taken with and without fluorescence. After applying this script, we could investigate variation in depletion over a range of cropped areas. Data points show median depletion values over a collection of values obtained for different crop areas. Blue error bars give standard deviation of the mean while black error bars show the spread between minimum and maximum depletion values.

pixel values (without pumping, with pumping, and with no atoms). We inferred error bars by propagating statistical errors accordingly.

For the measurements in Fig. 4.24, the power of the optical pumping beam was close to $75 \mathrm{~mW}$. The laser polarization was linear, although we did not operate the Helmholtz pairs for defining a quantization axis. We applied no spectral broadening to the beam (i.e. no power applied to the EOM). At the lowest temperature, we tuned the incidence angle and height of the optical pumping beam in order to minimize the integrated fluorescence beyond the magnets in the presence of pumping. Under these (non-optimal) conditions, we observe Li-6 suppression consistent with Li-7 enrichment to better than $99.95 \%$ (assuming no change to Li-7 throughput) up to $700{ }^{\circ} \mathrm{C}$. Even at the peak operating temperature, we suppress Li-6 throughput by close to a factor of 100 (yielding Li-7 enrichment to beyond 99.9\%).

Upon subsequent analysis of the original images that we acquired for Fig. 4.24, 


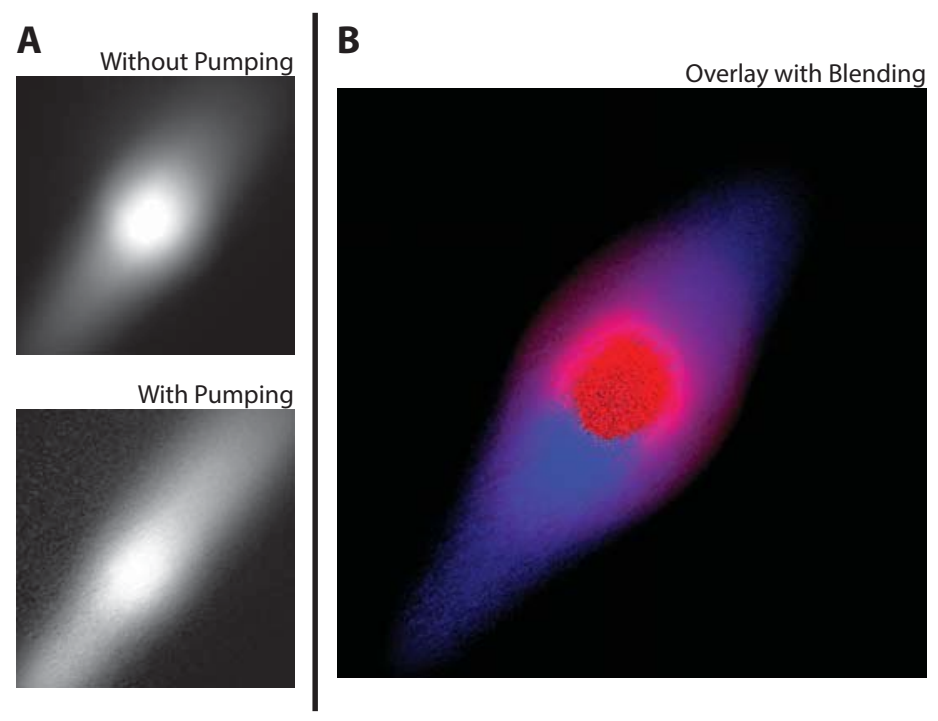

Figure 4.25: Outline of procedure for processing fluorescence images. (A) By enhancing the contrast on averaged (background-subtracted) images with and without optical pumping (corresponding to the measurement at $750{ }^{\circ} \mathrm{C}$ on Fig. 4.24 ) to saturate $1 \%$ of pixels, we observe that radiation pressure has offset the Li-6 distribution at the collection plane. We therefore use Adobe Photoshop CS6 to independently crop the original images to center the fluorescence (with a horizontal orientation) within a maximal area. (B) Overlaying the enhanced images in (A) with a blending effect clearly indicates that radiation pressure has offset the Li-6 distribution. 
we identified a more thorough scheme for extracting depletion factors. Fig. 4.25(A) shows averaged images (background-subtracted) with and without optical pumping at $750{ }^{\circ} \mathrm{C}$ after applying unique gain to each image in order to saturate $1 \%$ of pixels on both images. Overlaying the resulting images as shown in Fig. 4.25(B), we see that the fluorescence shifts slightly in the presence of optical pumping (likely due to radiation pressure offsetting the angular distribution incident on the magnet array).

The overlay suggests that we should derive the depletion factor by defining unique cropping areas for images with and without optical pumping that center the fluorescence in both cases. We devised a script in Adobe Photoshop CS6 - using the enhanced images in Fig. 4.25(A) - for batch processing all images. Besides centering the fluorescence for all images, the script also rotated the image to both yield a horizontal aspect ratio for the fluorescence (for simplifying analysis) and minimize area on the images contributing negligibly to the fluorescence. We applied this script twice to background images in order to define backgrounds corresponding to the unique cropped areas for the images with and without optical pumping.

Fig. 4.24(B) again calculates depletion factors as a function of temperature applying the previously described procedure, but now using the cropped areas derived from the original images. After updating the scheme for processing images, we readily examine variation in the depletion factor across the laser used for generating fluorescence. With the fluorescence now centered and horizontal, we use software (MathWorks MATLAB) to impose a grid on the images (centered around the fluorescence). We calculate the depletion factor within regions of increasing area defined by this grid, with every area including the brightest fluorescence at its center.

Using this procedure, we infer a collection of depletion factors for a given source temperature. The data points in 4.24(B) give the median depletion factor obtained by partitioning the image. While blue error bars in 4.24(B) give error derived from statistical uncertainties for the cropped area yielding this median value, the black bars show the range of depletion values for the areas considered by the grid that we imposed. The closest depletion value that we extract to the absolute depletion corresponds to the number that we infer from the largest crop area. We give the median value, however, 


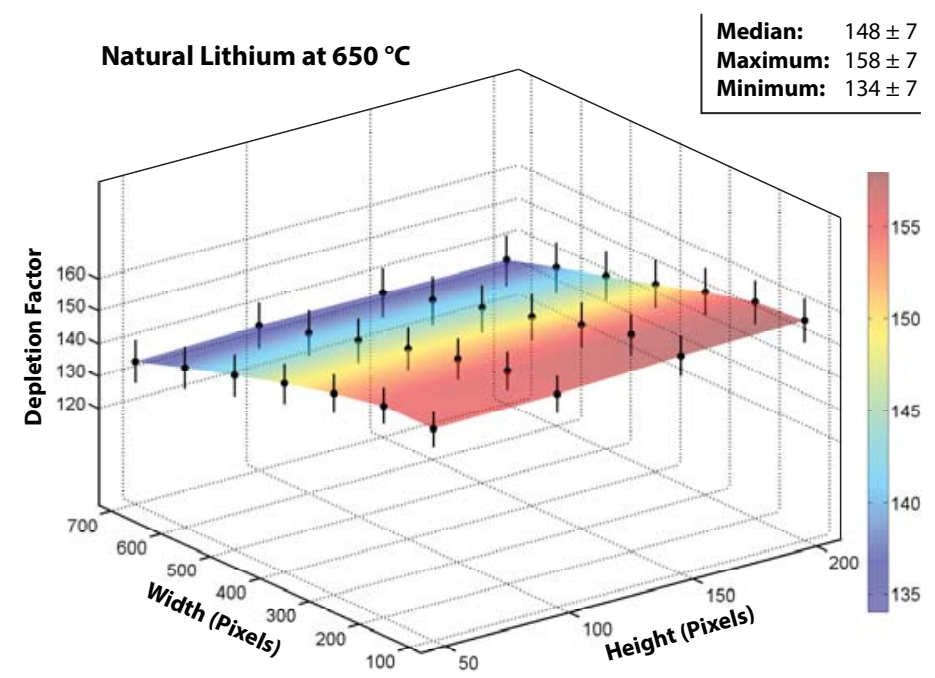

Figure 4.26: Overview of dependence of depletion value on cropping choice. After independently processing images taken in the presence and absence of optical pumping, we calculate depletion values over a range of cropped areas (all centered around the peak fluorescence). For this data set (corresponding to the measurement at $650{ }^{\circ} \mathrm{C}$ in Fig. 4.24), the depletion worsens for larger crop areas.

because the depletion is not uniform across the fluorescence. As intuition might suggest, Fig. 4.26 shows that the depletion (with the source at $650{ }^{\circ} \mathrm{C}$ ) worsens for larger crop areas. With the gradient across the fluorescence likely corresponding to an angular distribution of atomic trajectories (with power broadening dictating the angular extent that contributes to the fluorescence), however, spectral broadening on the optical pumping beam can yield better depletion over the entire fluorescence length.

Fig. 4.27 shows variation in Li-6 suppression as we vary laser power (A) and spectral broadening (B) with the source at $650{ }^{\circ} \mathrm{C}$. In order to not disturb the optical setup, we measure laser powers just prior to the spherical telescope on the lower platform for the beam line optics (in contrast to measurements made using the RGA, as described in Fig. 4.20). To avoid direct comparison to those earlier measurements, we give the laser powers in Fig. $4.27(\mathrm{~A})$ as fractional powers, where the maximum power is $78 \pm 4$ $\mathrm{mW}$ (with error corresponding to the accuracy of the calibration for an attenuator used on the power meter). Again, we reduce the laser power by installing neutral density filters in the path of the beam prior to the spherical telescope. We observe worsening of the depletion after attenuating the laser power to close to $40 \%$ of the peak power $(33 \pm 2$ 
$\mathrm{mW}$ ). Given the beam dimensions, this corresponds to an average beam intensity of close to $6 \mathrm{~mW} / \mathrm{cm}^{2}$, on par with the saturation intensity for the Li-6 D1 line. As expected, further reducing the laser power quickly worsens the depletion. We see insensitivity of the depletion to higher laser powers, indicating that we have maximized the transition rate on the D1 line over a sufficiently long interaction time to achieve optimal pumping into the $\mathrm{F}=1 / 2$ state.
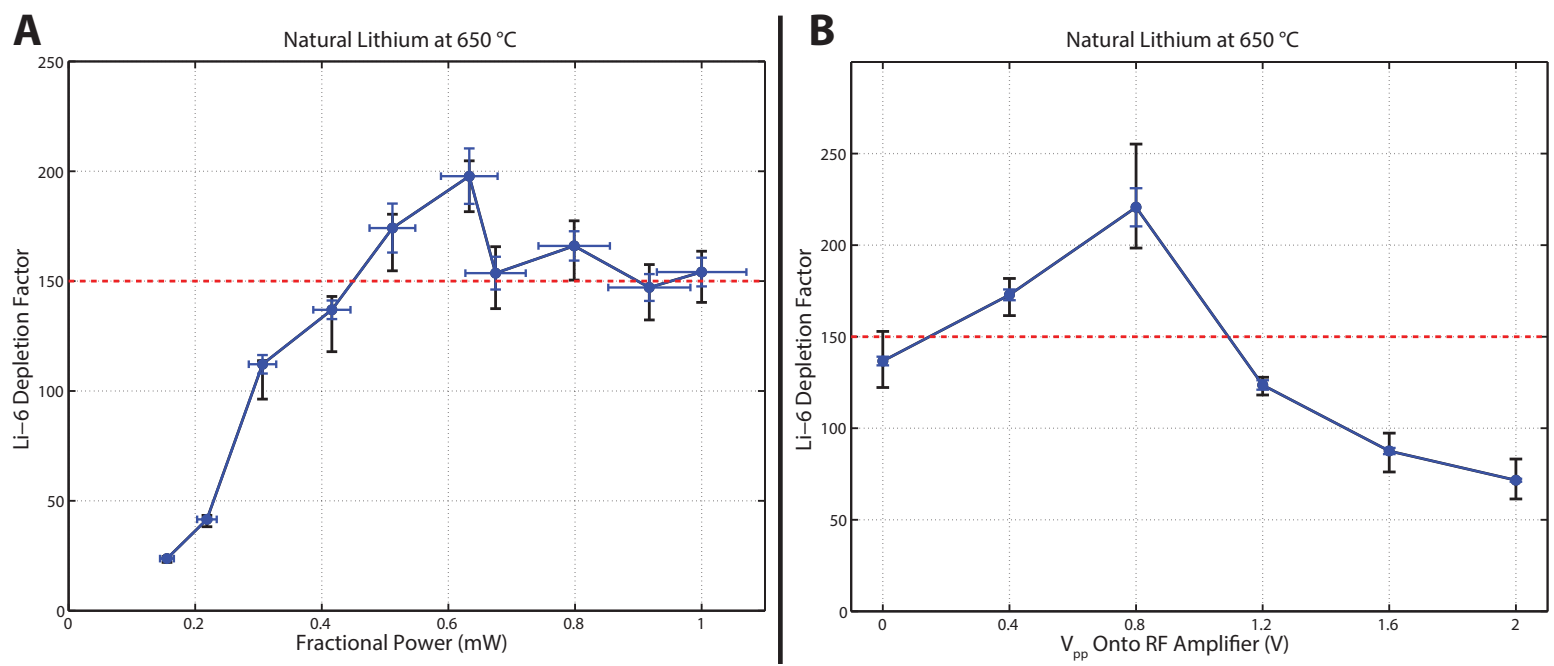

Figure 4.27: Depletion dependence on laser power and various powers applied to EOM (for natural lithium). (A) We measure laser powers prior to entry to the optical pumping cross. To avoid direct comparison to RGA measurements (where the power was measured after the cross), we give fractional powers. The peak laser power corresponds to $78 \pm 4$ $\mathrm{mW}$ and the minimum power is $12 \pm 1$. (B) During operation, we specify broadening according to the peak-to-peak voltage that we apply to an RF amplifier that drives the EOM. The amplifier effectively amplifies this voltage by $24.8 \mathrm{~dB}$ (amplifier gain is 44.8 $\mathrm{dB}$ and we attenuate the input by $20 \mathrm{~dB}$ ).

Fig. 4.27 shows the depletion as a function of power applied to the EOM. We took this data again at $650{ }^{\circ} \mathrm{C}$, but on a separate date than the data shown on Fig. 4.27(A) (after reloading the oven). We again used linear polarization without using the Helmholtz pairs. For these measurements, we measured the laser power to be $50 \mathrm{~mW}$ prior to the spherical telescope. We captured all images using $6 \mathrm{~s}$ exposures. In agreement with prior measurements using the RGA (see Fig. 4.20), depletion improves as we broaden the laser spectrum until reaching an optimal power on the EOM (in this case $0.48 \mathrm{~W}$ ). Beyond this point the depletion worsens, suggesting that the intensity per unit frequency 
has lowered to below saturation at the frequencies matching the relevant Doppler spread of the atomic beam in the pumping region. This data further indicates that depletion should improve with more laser power available and a broadening mechanism that more smoothly distributes power across frequencies (e.g. introducing noise directly onto the current driving the laser diode).

Fig. 4.28 compares results of the cropping procedure for a few data sets. The middle surface again shows the measurements from Fig. 4.26. The upper surface shows depletion values obtained using the same procedure applied to conditions very similar to those yielding the best depletion factor on Fig. 4.27(B) ${ }^{14}$. The depletion factor actually improves for this data as we expand the crop area. This gradient directly indicates that spectral broadening in fact expands the velocity distribution that the optical pumping beam addresses. With higher Li-6 suppression at the wings of the fluorescence, we deduce that the EOM has diverted more power to sidebands that efficiently pump velocity classes that more heavily intersect the collection plane adjacent to the peak throughput.

Fig. 4.29 compares the variation in depletion along the fluorescence beam using data corresponding to measurements from Fig. 4.27(B). For every pixel along the direction of the beam, we determine the mean pixel value along the perpendicular direction. Using these mean values, we obtain curves for the depletion factor along the direction of the fluorescence beam. Fig. 4.29(B) shows improving uniformity (orange then yellow) in the depletion across the beam until the depletion becomes higher at the wings (yellow the brown). For these three depletion measurements, the Li-6 suppression improves across the entire beam. As not all trajectories with common incidence angle on the magnet aperture will localize on the collection plane, we expect uniformly better suppression across the beam upon addressing a larger angular distribution of incident trajectories. The spatial extent on the collection plane should be determined by the thermal energy of the atoms, while the apparatus geometry governs the distribution of incidence angles.

\footnotetext{
${ }^{14}$ We used $\sigma^{-}$polarization in this case (defining a quantization axis accordingly) in an effort to truly optically pump atoms into the $\mathrm{F}=3 / 2, \mathrm{~m}_{\mathrm{F}}=-3 / 2$ state.
} 


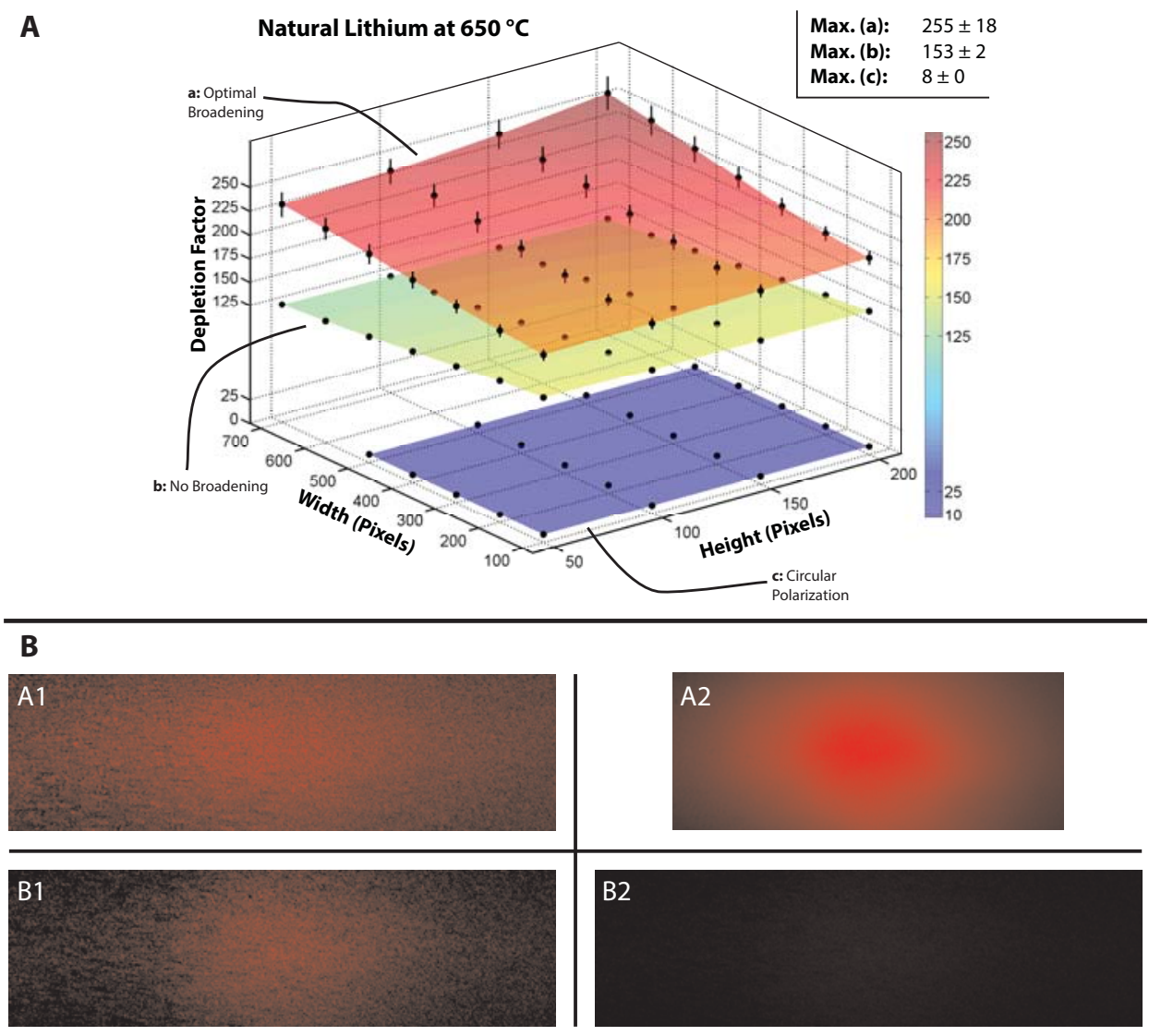

Figure 4.28: Summary of best and worst depletion values obtained from fluorescence measurements. (A) The middle surface corresponds to the same data described by Fig. 4.26. The upper surface shows depletion values extracted from optimized data. The lower surface corresponds to our worst measurements. In this case we try to polarize the Li-6 beam in the $\mathrm{m}_{\mathrm{F}}=+3 / 2$ state. (B) These images highlight the contrast between the fluorescence (with optical pumping applied) for the three data sets shown in (A). B1 and B2 both show the average (background-subtracted) image corresponding to the upper surface in (A) after applying different gains. The gain applied to B1 is the same as that applied to A1 (likewise for A2 and B2). A1 and A2 show average (backgroundsubtracted) images corresponding to the upper and lower surfaces, respectively. We choose gains for $\mathrm{A} 1 / \mathrm{B} 1$ and $\mathrm{A} 2 / \mathrm{B} 2$ that saturate the maximum intensity pixels in the original images corresponding to $\mathrm{A} 1$ and $\mathrm{A} 2$, respectively. 

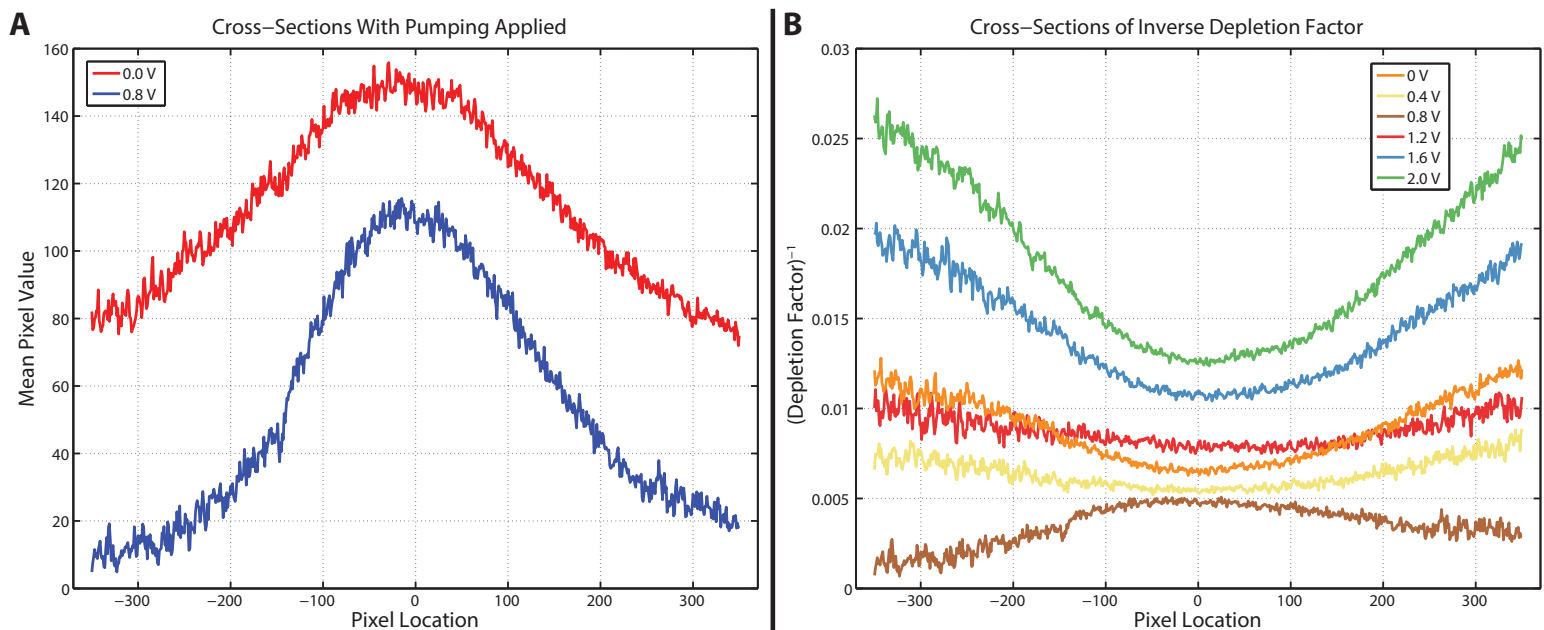

Figure 4.29: Spatial dependence of depletion for various powers applied to EOM. (A) Average pixel values along the fluorescence beam in the presence of optical pumping for a pair of RF powers applied to the EOM. (B) Depletion factors along the fluorescence beam for variety of RF powers applied to the EOM. Error bars not shown for the sake of not over-complicating the figures.

Beyond a certain RF power incident on the EOM, more power will be diverted from the central frequency and first-order sidebands to higher-order sidebands. While this should in principle ensure that we address a larger range of incidence angles, the intensity per unit frequency at certain frequencies will fall below saturation for certain RF powers. At sufficiently high RF power, the intensity will fall below saturation over a large frequency range. In fact, upon sufficiently increasing the incident RF power, Fig. 4.29 confirms that the suppression worsens across the entire florescence beam (brown then red then blue then green). At the highest RF powers the depletion reverts to being highest at the peak fluorescence, suggesting that the intensity at the central laser frequency is again higher than at the sidebands that address those incidence angles that contribute heavily at the wings of the fluorescence. With more intensity contributing to higher-order sidebands, however, the depletion factor around the peak fluorescence is worse than that obtained at lower RF powers (consistent with the hypothesis that the intensity across the relevant spectral extent has fallen too far below saturation).

The lowest surface on Fig. 4.28(A) shows a sample depletion measurement upon tuning the optical pumping beam to actually polarize the atomic beam in the 
$\left|F=3 / 2, m_{F}=-3 / 2\right\rangle$ state. In particular, we used a quarter-wave plate to prepare a circularly-polarized beam ${ }^{15}$. Preparing the quantization axis accordingly using the Helmholtz pairs, $\sigma^{+}$polarization prepared a large fraction of atoms in the $\left|F=3 / 2, m_{F}=+3 / 2\right\rangle$ state. As expected, Li-6 depletion worsened in this case as the $\left|F=3 / 2, m_{F}=+3 / 2\right\rangle$ state - which is high-field seeking - is dark with this selection rule. We still observed suppression because many Li-6 atoms (including some initially in low-field seeking states) will spontaneously decay into the entirely high-field seeking $\mathrm{F}=1 / 2$ state which is high-field seeking. With a repumping beam in the optical pumping chamber, we likely would have observed Li-6 enhancement.

\footnotetext{
${ }^{15}$ We temporarily introduced a polarizing beamsplitter cube in front of the waveplate. Using a power meter, we adjusted the waveplate to balance the powers at the outputs of the cube. We then removed the cube.
} 


\subsection{Extraneous Measurements}

A $\times 10^{14}$ Temperature Corrections for Source (G3)

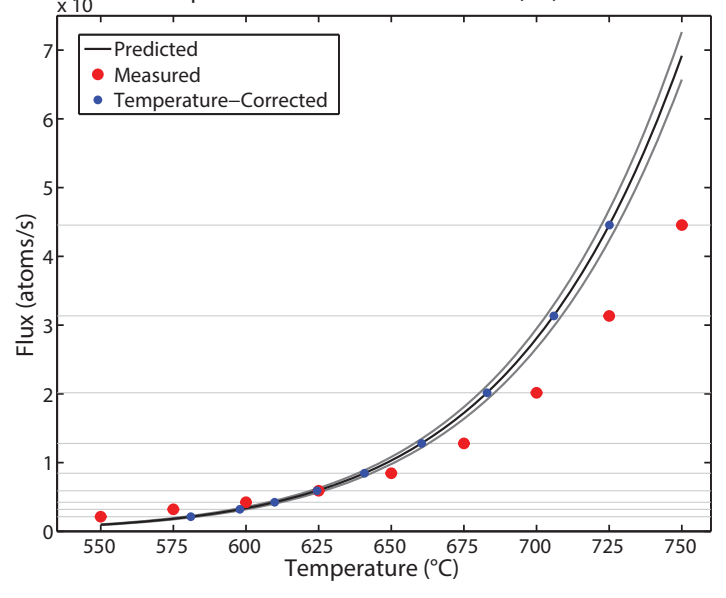

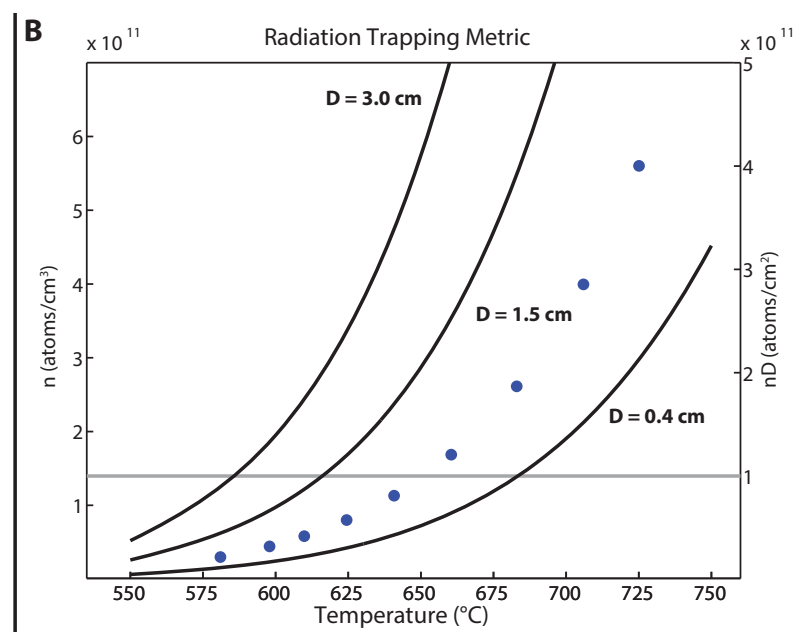

Figure 4.30: Estimates for atomic density in pumping region. (A) Deposition rates that we measure on the thickness monitor (from Fig. 4.24) give atomic flux over the sensor area (red). Comparing the resulting flux to flux predicted by kinetic gas theory (black, with gray curves conveying uncertainty in lithium vapor pressure), we impose corrections to the nominal temperatures that we previously measured via thermocouple. (B) Using the slightly offset temperatures, we can estimate the atomic number density (blue) in the pumping cross (particularly in the volume defined by the sensor diameter for the thickness monitor). Multiplying densities by the relevant atomic beam diameter (defined by intersection between atomic and pumping beams), we obtain a benchmark for determining the effect of radiation trapping on atomic polarization.

While the thickness monitor records deposition rates as film thickness per unit time, we can infer atomic flux per unit area simply by multiplying deposition rates by the atomic number density $n$ for lithium. For our effusive source operating at temperature $T$ and pressure $P$, the flux $I$ emitted through our aperture of area $A$ into solid angle $\Omega$ should be given by

$$
I=\left(\frac{P A}{\sqrt{2 \pi^{3} m k_{B}}}\right) \Omega
$$

where $m$ denotes the average mass of a lithium atom $[86]^{16}$. Fig. 4.30(A) compares the measured atomic flux (shown initially in Fig. 4.24) to that predicted by (4.8). Using

\footnotetext{
${ }^{16}$ The pressure should be given as the pressure at the source aperture which can be related to the pressure in the source using geometrical factors [87].
} 
the curve generated by (4.8), we applied corrections to the nominal source temperatures where we measured flux.

While the discrepancies between data and theory might be due to real phenomena (such as collisional effects in the source), we apply this correction in order to estimate the atomic density in the pumping region. Using these slightly offset temperatures, we infer the atomic density (via the Maxwell-Boltzmann distribution) by integrating over velocities $v$ to obtain

$$
n=2 I \int_{0}^{\infty} v^{2} \exp \left(-v^{2} / \alpha^{2}\right) d v /\left(\alpha^{4} A\right)
$$

where

$$
\alpha \equiv \sqrt{\left(2 k_{B} T / m\right)}
$$

and here $A$ denotes the cross-sectional area for the atomic beam in the pumping volume [56]. As shown in Fig. 4.30(B), the density in the pumping cross varies between $5 \times 10^{10}$ and $6 \times 10^{11}$ atoms $/ \mathrm{cm}^{3}$. Fig. 4.30(B) also shows curves for the product of $n$ with several diameters $D$ that physically denote the diameter of the intersection between the atomic and pumping beams. Prior work has shown that when the product $n D$ exceeds $10^{11}$ atoms $/ \mathrm{cm}^{2}$, atomic polarization begins to worsen. In fact, for the curve in Fig. 4.30(B) given by the lowest value for $D$ (corresponding to the relevant beam diameter that is incident upon the magnets), this threshold occurs close to where we observe worsening Li-6 depletion in Fig. 4.24.

In Chapter 2, we argued against optical pumping on the Li-6 D2 line due to the prominent transition between the $F= \pm 3 / 2$ and $F^{\prime}= \pm 5 / 2$ states. As shown in Fig. 4.31, we rapidly confirmed that working on the D2 line yields substantially worse Li-6 suppression than operating on the D1 line. In fact, due to this cycling transition, we suppressed Li-6 throughput in this case to just $\sim 75 \%$ of its throughput in the absence of pumping. Radiation pressure likely contributed substantially to this suppression.

For all measurements of Li-6 suppression, we imaged fluorescence in the pumping region onto a CCD in order to gain physical insight. As expected, we always generated a gradient in fluorescence across the laser along the atomic beam direction as the Li-6 


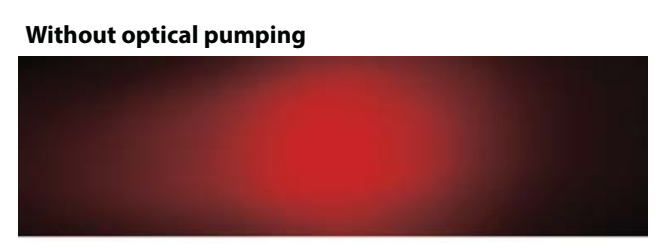

With optical pumping
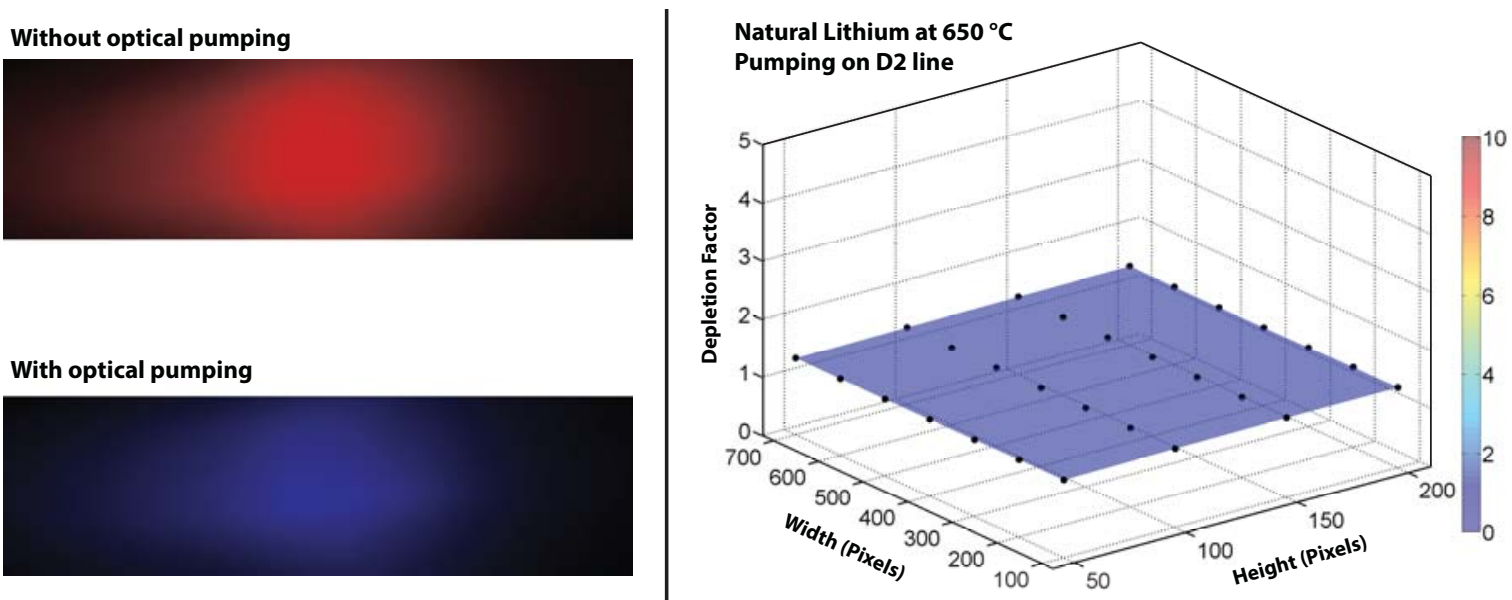

Figure 4.31: Sample depletion when pumping on the D2 line. Due to the very strong transition on the D2 line between the $F= \pm 3 / 2$ and $F^{\prime}= \pm 5 / 2$ states, we observe almost no Li-6 suppression. The images on the left (taken at identical exposures) show marginal Li-6 depletion by eye.

population in the $F=1 / 2$ ground state increased. Fig. 4.32 shows sample (backgroundsubtracted) fluorescence that we measured while recording data for the point at $750{ }^{\circ} \mathrm{C}$ in Fig. 4.24. A single-pass through the pumping region generated half the fluorescence shown in the image (symmetric about the center-line for the atomic beam). Each half of the fluorescence shows a pair of bright features that correspond to transitions from the $F=3 / 2$ ground state to the $F^{\prime}=1 / 2$ and $F^{\prime}=3 / 2$ excited states (with the outer feature corresponding to the former).

Using the CCD, we aligned the retroflection for the optical pumping beam in order to achieve symmetric fluorescence. Cross-sections along various directions on these fluorescence images provide further evidence for our hypotheses concerning the limiting factors for Li-6 depletion. For example, cross-sections along the atomic beam direction yield higher pixel values at the tail of the fluorescence as we increase source temperature. By looking at cross-sections perpendicular to the atomic beam, we can infer the Doppler width that the pumping beam addresses. Images clearly indicate that spectral broadening (applied via the EOM) enhances the angular distribution that interacts appreciably with the laser. Upon increasing by broadening, however, the contrast in the fluorescence along the atomic beam direction worsens, indicating poorer atomic polarization. 


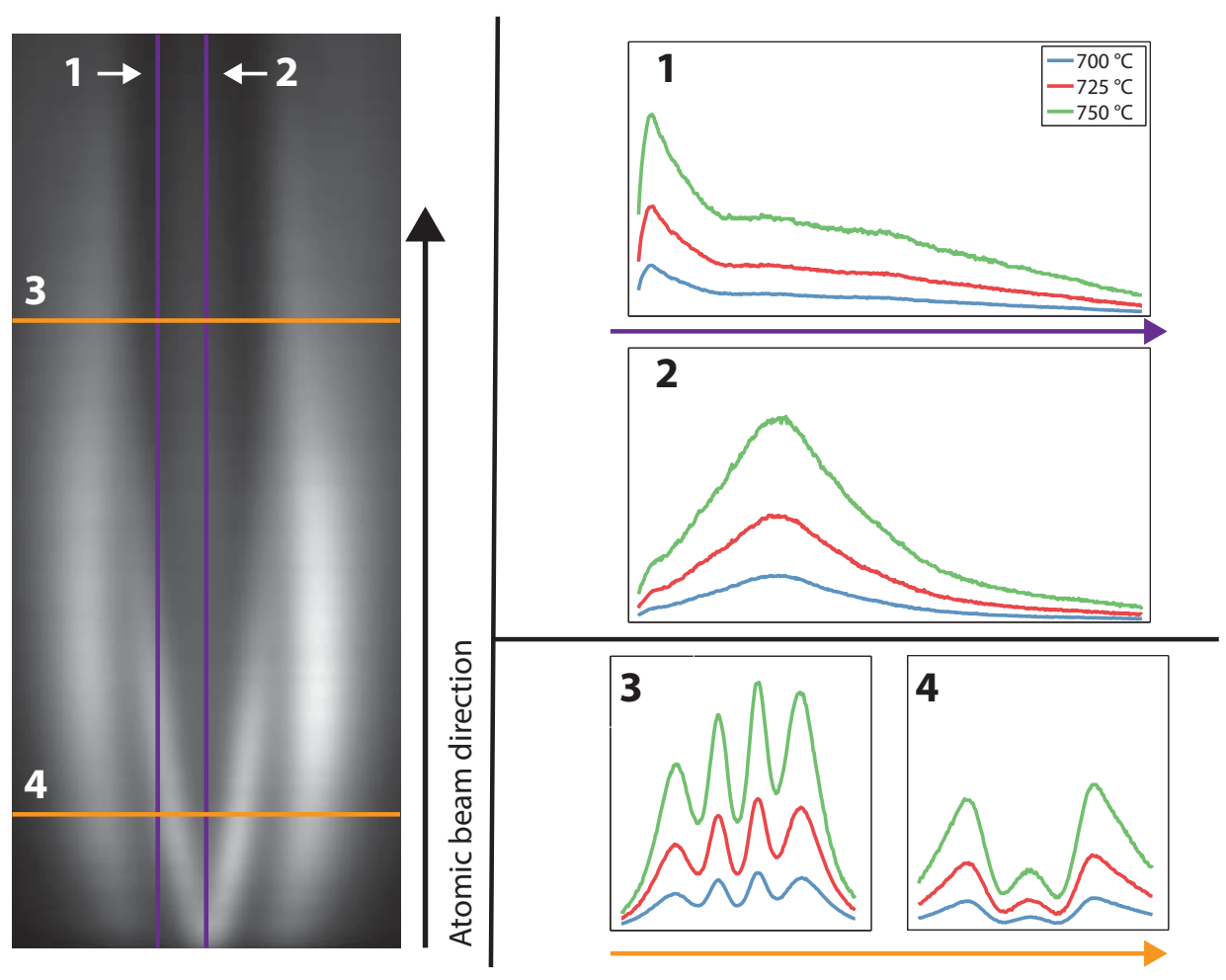

Figure 4.32: Sample fluorescence measured from above the optical pumping cross on a CCD. We use fluorescence images taken in the pumping cross as diagnostics for corroborating hypotheses. We captured the image on the left when measuring Li- 6 suppression for the data point in Fig. 4.24 at $750{ }^{\circ} \mathrm{C}$. Cross-sections along various directions show effects like worsening atomic polarization at higher operating temperatures. 


\section{Chapter 5}

\section{Apparatus Scaling, Beyond Lithium, and Conclusions}

Fig. 5.1 summarizes key results of our proof-of-principle experiment. Using a single, 1.5 in tall magnet array we measured fluxes of $(7.2 \pm 0.4) \times 10^{11}$ and $(1.34 \pm 0.02) \times$ $10^{12}$ atoms $/ \mathrm{mm} \cdot \mathrm{s}$ at nominal source temperatures of $650 \mathrm{C}$ and $700{ }^{\circ} \mathrm{C}$, respectively. By extending the height of this array, this guide design should yield $0.8 \pm 0.1$ and $1.6 \pm 0.2$ grams per vertical meter of guide entrance per continuous year of operation. To thus yield Li-7 throughput on par with the figure-of-merit for calutron flux $(\sim 0.1$ mol per day weighted by relative isotope abundance), a commercial apparatus would require 50 of these guides with each guide being $3 \mathrm{~m}$ tall. While these numbers appear daunting, the permanent magnets are inexpensive and require no energy input. As shown previously in Figs. 3.15, 3.18, 3.19, and 3.20, we have investigated several viable methods for arranging the magnets including mechanical fastening and epoxying. Bruce Klappauf has investigated the prospects for using superconducting coils in place of permanent magnets (in order to make more compact assemblies). In addition, recent research -

including the development of thermomagnetically patterned micromagnets - has shown promising alternatives to bulk rare-earth permanent magnets $[88,89]$.

The source on a commercial apparatus will be engineered to both maximize usable flux and minimize power requirements. While we hastily built the source for this work, a plethora of literature exists on the subject of constructing beam sources for metallic atoms [87]. Rather than pivoting the source, guides can pivot individually about points on a radius defining the source-to-guide distance. Optics between guides including prisms should allow a single laser to be used for the optical pumping of atoms incident on all guides. On a commercial apparatus we will position targets accordingly (either beyond guides or along the magnet surfaces) for collecting enriched material. Detectors like those that we used for characterizing lithium throughput can be implemented for dynamically 
optimizing the apparatus performance.

Both our RGA and fluorescence measurements indicate Li-6 suppression beyond a factor of 200 (at source temperatures up to $650{ }^{\circ} \mathrm{C}$ ) which in turn implies Li-7 enrichment to better than $99.95 \%$. In both cases, we improved Li-6 suppression by applying spectral broadening to the optical pumping beam using an electro-optic modulator. We observed maximum suppression (corresponding to a factor of close to 250) when broadening the spectrum to between 20 and $30 \mathrm{MHz}$ (full-width at half-maximum) ${ }^{1}$. Upon broadening further, Li-6 suppression worsened with fluorescence measurements suggesting uniform worsening across the entire collection plane. We therefore believe that Li-6 suppression was limited due to having inadequate power per unit frequency for addressing the Doppler spread of the portion of the atomic beam incident on the magnet aperture. As long as an optical pumping beam has a sufficiently high saturation parameter over adequate spatial and frequency extents, the resulting atomic polarization incident on all guides of a commercial apparatus should be comparable. For typical saturation intensities, fractional power losses due to absorption should be small between channels.

Li-6 suppression likely worsened at the highest source temperatures that we considered due to radiation trapping. Between nominal source temperatures of 550 and 750 ${ }^{\circ} \mathrm{C}$, flux measurements in the pumping region indicate that the atomic density $n$ in this region varied approximately between $1 \times 10^{10}$ and $5 \times 10^{11}$ atoms $/ \mathrm{cm}^{3}$. Prior theoretical work showed that when the product $n D$ - with $D$ denoting the atomic beam diameter determined by the intersection of the optical pumping laser and the atomic beam - exceeds $10^{11}$ atoms $/ \mathrm{cm}^{2}$, radiation trapping begins to contaminate atomic polarization [56]. Given the relevant diameter for the atomic beam in the pumping region, our apparatus reaches this criterion between 650 and $700{ }^{\circ} \mathrm{C}$, exactly where we first notice worsening of Li-6 suppression. We likely can mitigate this effect by performing optical pumping further away from the source where atomic density has lessened at a particular temperature $^{2}$. Moving away from the source, however, introduces technical complexity

\footnotetext{
${ }^{1}$ See Fig. 3.12 for measurements of the spectral broadening using a Fabry-Perot interferometer.

${ }^{2}$ The density will lessen faster than the atomic beam diameter grows.
} 


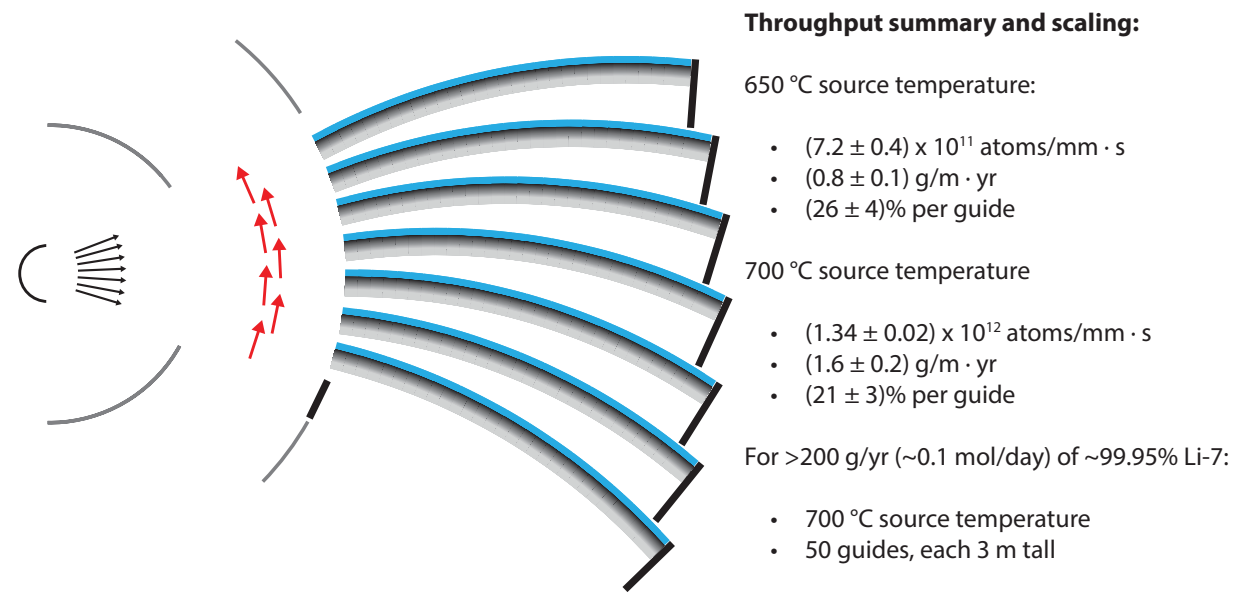

Figure 5.1: Throughput summary and apparatus scaling. To produce higher throughputs, we simply extend the height for the magnetic guide. We then position identical guides around the source.

as the height for the pumping beam will need to increase in order to match the vertical extent of the atoms.

Application of MAGIS to other elements will proceed similarly to the experiment described in this work. The principle underlying the design for the guide curvature will likely remain the same for other elements. By using an aperture and curved guide, atoms will have no line-of-sight from the source to a collection plane as long as the source is positioned accordingly. By tailoring the guide curvature such that all trajectories originating at a point source impinge on the guide at the same angle, the geometry determines a maximum speed for trajectories that can be deflected to beyond the magnets. The aperture width selects the transverse velocity distribution incident on the guide, and in turn sets the minimum length for the guide (for a particular source-to-guide distance and curvature). Tuning the guide curvature and choosing other parameters accordingly, we can compromise apparatus performance and scale for a given element.

As the source temperature determines the velocity distribution incident on the magnet arrays, a given curvature will work better for lower temperature elements. For example, while the magnet panel used in this work enabled $50 \%$ guiding efficiency for low-field seeking lithium atoms, the same panel should reflect the entire velocity distribution for mercury whose vapor pressure is $1 \mathrm{~Pa}$ at just $200{ }^{\circ} \mathrm{C}$. Fig. 5.2 gives operating 


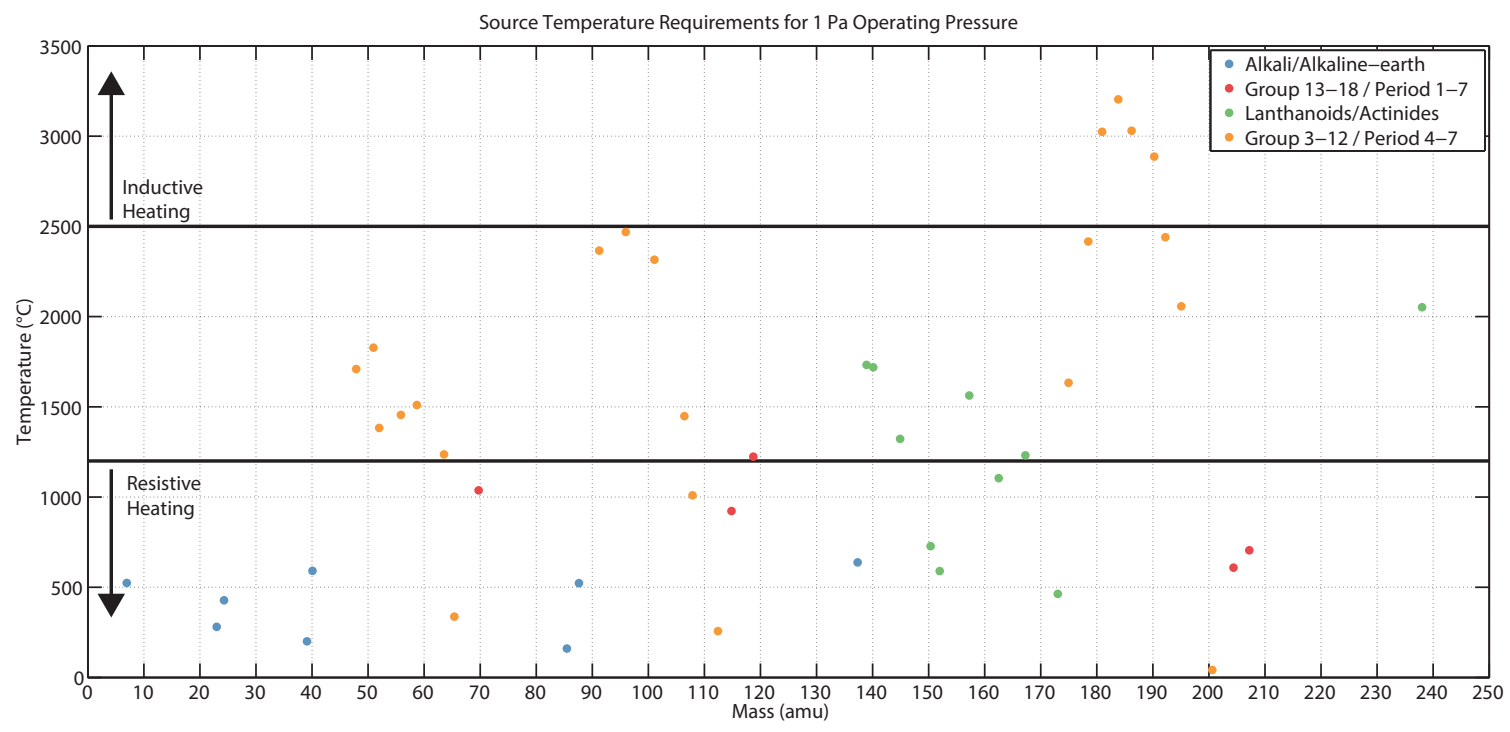

Figure 5.2: Comparison of vapor pressures among metallic elements. Data points show necessary temperatures for producing $1 \mathrm{~Pa}$ vapor pressures.

temperatures necessary (among candidate elements) for achieving $1 \mathrm{~Pa}$ vapor pressures in a source. Lithium requires one of the higher operating temperatures among both the alkali and alkaline-earth metals. Nearly all of these elements will operate at temperatures below $1000{ }^{\circ} \mathrm{C}$. With care, resistively heated sources work effectively and efficiently at temperatures up to $1000{ }^{\circ} \mathrm{C}$. The highest temperature elements correspond to refractory metals - notably including tungsten, tantalum, rhenium and osmium - that will operate beyond $2500{ }^{\circ} \mathrm{C}$. These metals will need to be heated directly by either electron bombardment or induction in a crucible like graphite or boron nitride [87]. To compensate for the faster speed distributions in the case of refractory metals, the guide might need to be lengthened for accommodating a shallower curvature.

While the guide design will translate to other elements, the exact layout for the guide will depend on the isotope of interest. For elements having more than two isotopes, the apparatus used for this work will not be sufficient for enriching a single isotope unless performing optical pumping on atoms of all undesired isotopes. Fig. 5.3 shows a more general field configuration - combining two arrays (identical in design to that used in this work) in series - that can be applied to any isotope. By properly 


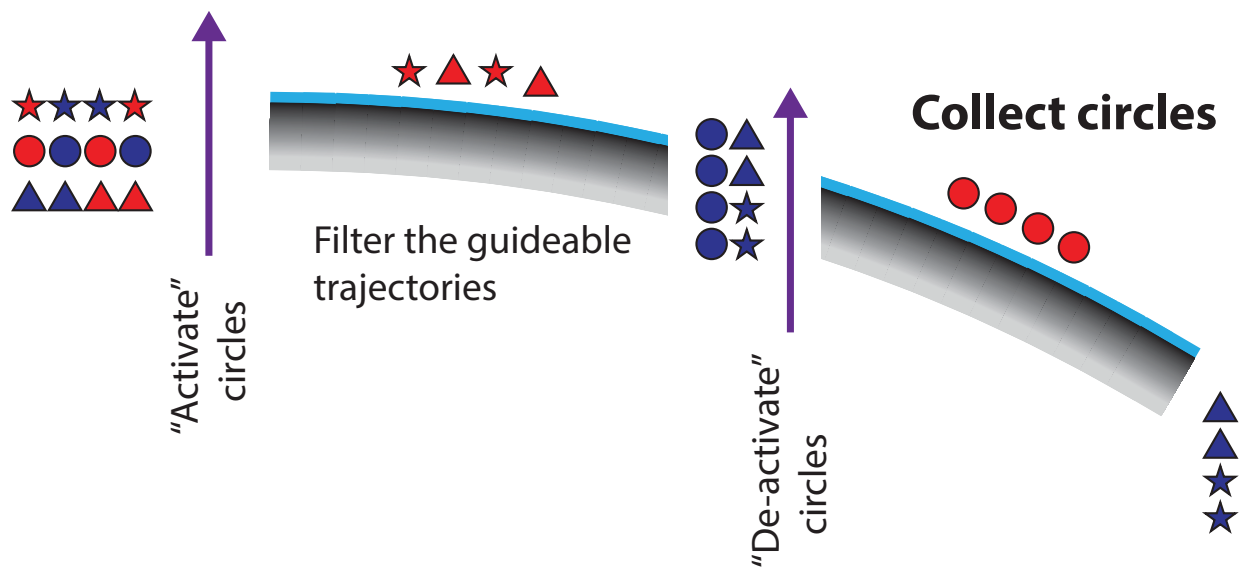

Figure 5.3: More general magnetic field configuration for enriching arbitrary isotopes. For elements with more than two isotopes, two guides in series can extract atoms of just one isotope. The first guide selects all guideable trajectories. By pumping atoms of the isotope of interest into a high-field seeking state between the guides, those atoms will collect on the second guide.

configuring the arrays, any trajectories that passes beyond the first array should reach beyond the second array. By pumping atoms of the targeted isotope into a high-field seeking state in the gap between the arrays, these atoms should collect on the face of the second array. Throughput for the isotope of interest can be enhanced by first pumping these atoms into a low-field seeking state prior to the first array. This setup introduces technical complexity as the optical pumping beam between arrays will need to be large enough to match the vertical extent of the atomic beam. Bruce Klappauf simulated the performance for this arrangement using several isotopes - including Ca-48 and Ni-64 and confirmed that the overall efficiency for collecting material should be comparable to the guiding efficiency for a single guide.

All isotopes will require unique optical pumping schemes. Optical pumping for all isotopes of a given element, however, should proceed among the same set of electronic states for that element. As summarized in Fig. 5.4 and Table 5.1, to date we have identified optical pumping schemes for 129 isotopes of 27 elements ${ }^{3}$. For isotopes with no nuclear spin (corresponding to most even-mass isotopes), optical pumping will drive atoms into a stretch state for a particular level within the fine structure. For certain iso-

\footnotetext{
${ }^{3}$ This list is not exhaustive. We expect to expand the list upon further investigating other elements.
} 
topes of various elements (like lithium and other alkali metals), pumping atoms between hyperfine states will be sufficient. For other elements, however, the presence of hyperfine structure might require auxiliary beams for sustaining the atomic population within a particular hyperfine level for optical pumping into a stretch state of that level. These additional frequencies, however, will most often be derivable from a single laser using frequency shifters. Most of the elements in Fig. 5.4 requiring multiple wavelengths - notably including the alkaline-earths, zinc, mercury, and ytterbium - have zero electronic magnetic moment in their ground state. An additional laser will be used to drive atoms of these elements into a long-lived metastable state along a narrow intercombination line. Optical pumping will then polarize atoms of a given isotope in a magnetic substate within this metastable state.

Literature provides a wealth of relevant spectroscopic information including saturation intensities (which in turn give transition cross-sections) and isotope shifts. Known saturation intensities for the transitions given in Table 5.1 range from below $1 \mathrm{~mW}$ to close to $100 \mathrm{~mW}$ (see references in Table 5.1). A lot of spectroscopic information provided by the references in Table 5.1 was derived from laser cooling experiments. In contrast to optical pumping, laser cooling requires many $\left(\sim 10^{5}\right)$ cycles along a given transition per atom. To achieve this cycling, these transitions typically require that $\Delta F=+1$ between ground and excited states. While using $\sigma^{+}$polarization enables cycling in this case, finite branching ratios will sometimes require additional lasers for re-pumping atoms into the ground state for the cooling line. Optical pumping will therefore benefit more by using transitions with $\Delta F=0,-1$ where atoms can more readily be polarized in a dark state using suitable laser polarization. For example, the most practical line for laser cooling iron (372 nm) decays to a metastable state that is no longer resonant with this transition after several hundred spontaneous emission events on average. Without using multiple lasers to circumvent this leakage, laser cooling is curtailed. In contrast, while optical pumping on this transition should be possible, a nearby transition at $368 \mathrm{~nm}$ with $\Delta J=0$ might be better suited for MAGIS.

Commercial solid-state laser systems are available that will provide at least 100 $\mathrm{mW}$ for almost all of the transition wavelengths given in Table 5.1. This power is gen- 
erally sufficient to separate approximately 5 moles per year of a desired isotope. Most commercial systems consist of readily available laser diodes and amplifiers ${ }^{4}$. An alternative option to this combination, particularly further in the UV, is an optically pumped semiconductor laser (OPSL). OPSLs achieve very high powers $(>1 \mathrm{~W})$ by optically pumping a solid-state gain region, typically using a high power pump at around $800 \mathrm{~nm}$. Controlling the fabrication of the gain region, emission wavelengths can be tailored to be between 900 and $1200 \mathrm{~nm}[90,91]$. Using a frequency doubling cavity (commercially available), over $500 \mathrm{~mW}$ between 450 and $600 \mathrm{~nm}$ can be produced. With a second stage of frequency doubling over $200 \mathrm{~mW}$ between 225 and $300 \mathrm{~nm}$ can be achieved [92, 93].

In conclusion, we have demonstrated Li-6 suppression in a thermal beam beyond a factor of 200. We likewise measured enriched Li-7 flux that naturally scales to commercially-relevant quantities simply by extending the apparatus dimensions. A machine that fits within several cubic meters should enable tens to hundreds of moles of material (depending on relative abundances of feedstock) to be enriched per year. In contrast to the calutron, MAGIS requires no prohibitive energy expense. The magnetic field gradient requires no power consumption, and optical pumping uses only low-power $(<1 \mathrm{~W})$ lasers. The atomic source and vacuum pumps will run continuously, but these are negligible energy expenses in comparison to that required for maintaining the static magnetic field for calutron operation ${ }^{5}$. Because of its broad applicability, combined with its attainable enrichment, throughput, and efficiency, we believe that MAGIS will help to mitigate the loss of isotope production due to the shutdown of the calutrons. In the near future, we think that MAGIS will be able to produce small quantities of isotopes particularly having medical applications. Looking further ahead, perhaps MAGIS will even evolve into operating plants providing isotopes like Li-7 for next-generation nuclear reactors or Hg-196 for more efficient fluorescent lighting.

\footnotetext{
${ }^{4}$ For example, Toptica Photonics provides systems across large wavelength range. See http://www.toptica.com.

${ }^{5}$ Moreover, these are shared expenses.
} 


\begin{tabular}{|c|c|c|c|c|c|c|c|c|}
\hline 茥 & $=\frac{0}{2}$ & Ф文 & $\because ‡$ & $\dot{n} \ddot{x}$ & 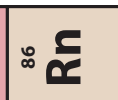 & & ב & د دٌ \\
\hline \multirow{5}{*}{ 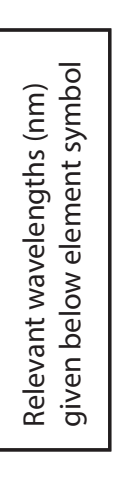 } & वL L & $\therefore \bar{U}$ & $\cong \bar{m}$ & $\stackrel{n}{n}-$ & 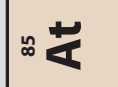 & & $\therefore \frac{0}{2}$ & $\stackrel{0}{2}$ \\
\hline & $\infty 0$ & $\because \backsim$ & m. & $\tilde{n} \mathscr{U}$ & \pm 0 & & $: \varepsilon$ & $=\frac{0}{2}$ \\
\hline & $-\mathbf{Z}$ & $\because 0$ & m & in & $\approx \bar{\infty}$ & & : & 토 \\
\hline & $\cdot U$ & $\pm \bar{n}$ & $\approx$ & in & $\approx \frac{0}{\alpha}$ & & 웅 & ริ \\
\hline & $n$ & $m$ & $\bar{m}$ & q $\subseteq$ 害 & $\bar{\infty} \boldsymbol{F}$ & & : & œせ \\
\hline \multirow{5}{*}{ 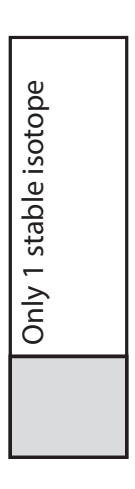 } & \multirow{5}{*}{$\begin{array}{l}\tilde{0} \\
0 \\
0 \\
0 \\
0\end{array}$} & \multirow{5}{*}{ 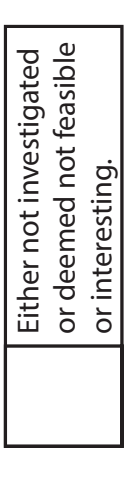 } & 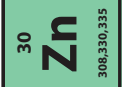 & \% ర్ & 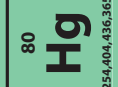 & & $\because \frac{0}{1}$ & مू \\
\hline & & & $2 \mathbf{3}$ & $=8$ & $\therefore \frac{2}{4}$ & E & × $\mathrm{O}$ & : \\
\hline & & & $\approx \overline{\mathbf{Z}}$ & ס & $\stackrel{2}{2}$ & $\stackrel{\circ}{\cong}$ & ¿ & ถู $\frac{\varepsilon}{<}$ \\
\hline & & & $\approx 0$ & ${ }^{8} \frac{\text { व }}{\alpha \simeq}$ & $\approx$ 느 & $\stackrel{+}{\Sigma}$ & $\frac{E}{n}$ & ะ \\
\hline & & & 닌 & $\ddagger \underset{\boldsymbol{\alpha}}{\boldsymbol{\alpha}}$ & ㅇ̆ & $\stackrel{n}{\circ} \frac{n}{1}$ & $-\frac{\xi}{Q}$ & 을 \\
\hline \multirow{6}{*}{ 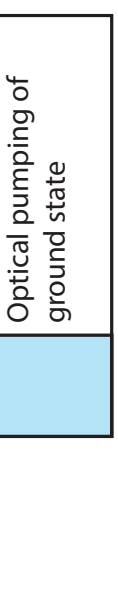 } & \multirow{3}{*}{ 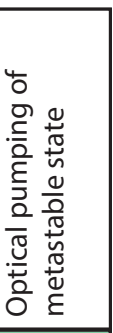 } & \multirow{4}{*}{ 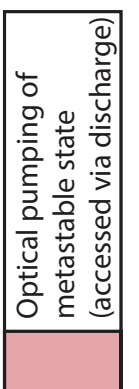 } & ${ }^{n} \frac{\Sigma}{\Sigma}$ & ${ }^{7} \boldsymbol{\sim}$ & ${ }^{n} \underset{\mathscr{x}}{\boldsymbol{\mu}}$ & 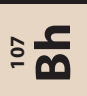 & $: \frac{\mathbf{0}}{\mathbf{Z}}$ & כ \\
\hline & & & ¿さう & 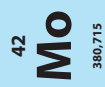 & \pm 3 & $\stackrel{\circ}{\circ}$ & in & $\bar{\sigma}$ \\
\hline & & & $\approx>$ & $=\frac{0}{Z}$ & ${ }^{n} \mathbb{0}$ & $\stackrel{0}{\circ}$ & U & ع \\
\hline & & & $\therefore \mathrm{F}$ & ì̀ & $\therefore$ 蒫 & 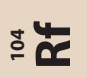 & is & D \\
\hline & & & $=\tilde{n}$ & $i>$ & & & & \\
\hline & + & $\simeq \sum^{\circ} \frac{n_{2}^{2}}{2}$ & : & ๓ & : & ฌ & & \\
\hline -I & ב" ב"m & $=\frac{\pi}{Z}$ & $\simeq \simeq$ & in & $\because \tilde{y}$ & œ亡 & & \\
\hline
\end{tabular}

Figure 5.4: Summary of isotopes identified thus far as candidates for MAGIS. See Table 5.1 for more details for each element, including references providing relevant spectroscopic information. 


\begin{tabular}{|c|c|c|c|c|c|}
\hline & Element & Stable Isotopes & Target State & $\lambda(\mathbf{n m})$ & Refs. \\
\hline 1 & $\mathrm{Li}$ & 6,7 & $2 s{ }^{2} \mathrm{~S}_{1 / 2}$ & 671 & {$[41,42]$} \\
\hline 2 & $\mathrm{Mg}$ & $24,25,26$ & $3 s 3 p{ }^{3} \mathrm{P}_{2}(2050 \mathrm{~s})$ & $457,383,384$ & {$[94-97]$} \\
\hline 3 & Ar & $36,38,40$ & $3 s^{2} 3 p^{5}\left({ }^{2} \mathrm{P}_{3 / 2}\right) 4 s{ }^{3} \mathrm{P}_{2}(60 \mathrm{~s})$ & 811 & {$[51,98]$} \\
\hline 4 & $\mathrm{~K}$ & $39,40,41$ & $4 s{ }^{2} \mathrm{~S}_{1 / 2}$ & 770 & {$[42,99]$} \\
\hline 5 & $\mathrm{Ca}$ & $40,42,43,44,46,48$ & $4 s 4 p{ }^{3} \mathrm{P}_{2}(7000 \mathrm{~s})$ & $657,443,445$ & {$[94-96,100,101]$} \\
\hline 6 & $\mathrm{Cr}$ & $50,52,53,54$ & $3 d^{5}\left({ }^{6} \mathrm{~S}\right) 4 s a^{7} \mathrm{~S}_{3}$ & 425 & {$[102,103]$} \\
\hline 7 & $\mathrm{Fe}$ & $54,56,57,58$ & $3 d^{6} 4 s^{2} a^{5} \mathrm{D}_{4}$ & 372 & {$[104,105]$} \\
\hline 8 & $\mathrm{Ni}$ & $58,60,61,62,64$ & $3 d^{8}\left({ }^{3} \mathrm{~F}\right) 4 s^{2}{ }^{3} \mathrm{~F}_{4}$ & 323 & {$[106]$} \\
\hline 9 & $\mathrm{Cu}$ & 63,65 & $3 d^{10} 4 s{ }^{2} \mathrm{~S}_{1 / 2}$ & 327 & {$[107]$} \\
\hline 10 & $\mathrm{Zn}$ & $64,66,67,68,70$ & $3 d^{10} 4 s 4 p{ }^{3} \mathrm{P}_{2}(>100 \mathrm{~s})$ & $308,330,335$ & {$[108]$} \\
\hline 11 & $\mathrm{Ga}$ & 69,70 & $4 s^{2} 4 p^{2} \mathrm{P}_{3 / 2}(-)$ & 403,294 & {$[109-111]$} \\
\hline 12 & $\mathrm{Kr}$ & $78,80,82,83,84,86$ & $4 s^{2} 4 p^{5}\left({ }^{2} \mathrm{P}_{3 / 2}\right) 5 s{ }^{3} \mathrm{P}_{2}(85 \mathrm{~s})$ & 811 & {$[51,112]$} \\
\hline 13 & $\mathrm{Rb}$ & 85,87 & $5 s{ }^{2} \mathrm{~S}_{1 / 2}$ & 795 & {$[42,113]$} \\
\hline 14 & $\mathrm{Sr}$ & $84,86,87,88$ & $5 s 5 p^{3} \mathrm{P}_{2}(1000 \mathrm{~s})$ & $689,679,688,707$ & {$[94-96,101,114,115]$} \\
\hline 15 & Mo & $92,94,95,96,97,98,100$ & $4 d^{5}\left({ }^{6} \mathrm{~S}\right) 5 s a^{7} \mathrm{~S}_{3}$ & 380,715 & {$[116-118]$} \\
\hline 16 & $\mathrm{Ag}$ & 107,109 & $4 d^{10}\left({ }^{1} \mathrm{~S}\right) 5 s{ }^{2} \mathrm{~S}_{1 / 2}$ & 328 & {$[119]$} \\
\hline 17 & $\mathrm{Cd}$ & $106,108,110,111,112,113,114,116$ & $5 s 5 p{ }^{3} \mathrm{P}_{2}(>10 \mathrm{~s})$ & $326,346,361$ & {$[120]$} \\
\hline 18 & In & 113,115 & $5 p^{2} \mathrm{P}_{3 / 2}(-)$ & 410,451 & {$[121,122]$} \\
\hline 19 & $\mathrm{Xe}$ & $124,126,128,129,130,131,132,134,136$ & $5 p^{5}\left({ }^{2} \mathrm{P}_{3 / 2}\right) 6 s{ }^{3} \mathrm{P}_{2}(150 \mathrm{~s})$ & 881 & {$[51,123]$} \\
\hline 20 & $\mathrm{Ba}$ & $130,132,134,135,136,137,138$ & $6 s 5 d{ }^{3} \mathrm{D}_{2}(60 \mathrm{~s})$ & $326,347,361$ & {$[30,124]$} \\
\hline 21 & $\mathrm{Nd}$ & $142,143,144,145,146,148,150$ & $4 f^{4} 6 s^{2}{ }^{5} \mathrm{I}_{4}$ & 472 & {$[125-127]$} \\
\hline 22 & Gd & $152,154,155,156,157,158,160$ & $4 f^{7}\left({ }^{8} \mathrm{~S}\right) 5 d 6 s^{2}{ }^{9} \mathrm{D}_{6}$ & 423 & {$[128]$} \\
\hline 23 & Dy & $156,158,160,161,162,163,164$ & $4 f^{10} 6 s^{2}{ }^{5} \mathrm{I}_{8}$ & 421 & {$[129-132]$} \\
\hline 24 & Er & $162,164,166,167,168,170$ & $4 f^{12} 6 s^{2}{ }^{3} \mathrm{H}_{6}$ & 401 & {$[132-134]$} \\
\hline 25 & $\mathrm{Yb}$ & $168,170,171,172,173,174,176$ & $4 f^{14}\left({ }^{1} \mathrm{~S}\right) 6 s 6 p{ }^{3} \mathrm{P}_{2}(12 \mathrm{~s})$ & $556,458,494$ & {$[94,135,136]$} \\
\hline 26 & $\mathrm{Hg}$ & $196,198,199,200,201,202,204$ & $5 d^{10}\left({ }^{1} \mathrm{~S}\right) 6 s 6 p{ }^{3} \mathrm{P}_{2}(7 \mathrm{~s})$ & $254,404,436,365$ & {$[137,138]$} \\
\hline 27 & $\mathrm{Tl}$ & 203,205 & $6 s^{2} 6 p^{2} \mathrm{P}_{3 / 2}(0.15 \mathrm{~s})$ & 378,352 & {$[139,140]$} \\
\hline
\end{tabular}

Table 5.1: Optical pumping details for 27 elements, corresponding to 129 isotopes. Target states correspond to the ground states of the chosen optical pumping transitions. In cases where this is not the ground state of the atom, the state lifetime is given in parentheses (if found in literature). References point to relevant spectroscopic data including isotope shifts, hyperfine splittings, and cross-sections. Note that excitation to metastable states for noble gases requires a discharge. 


\section{Bibliography}

[1] T.R. Mazur, B. Klappauf, and M.G. Raizen. Demonstration of magnetically activated and guided isotope separation. Nature Physics, 10:601, 2014.

[2] E.A. Symons. Lithium Isotope Separation: A Review of Possible Techniques. Separation Science and Technology, 20:633, 1985.

[3] D.P. Jackson, W.N. Selander, B.M. Townes, T.C. Leung et al. A Review of Fusion Breeder Blanket Technology. Canada Fusion Cells Technology Project (Report No. CFFTP-G-84033), 1985.

[4] Managing Critical Isotopes: DOE's Isotope Program Needs Better Planning for Setting Prices and Managing Production Risks. United States Government Accountability Office (http://www.gao.gov/products/GAO-12-591), 2011.

[5] Isotopes for the Nation's Future: A long range plan. Nuclear Science Advisory Committee (http://science.energy.gov//media/np/nsac/pdf/docs/nsaci_ii_report.pdf), 2009.

[6] James Howard Hinderer. Radioisotopic Impurities in Promethium-147 Produced at the ORNL High Flux Isotope Reactor. Masters thesis, University of Tennessee, 2010 .

[7] J.E. Lawler and M.G Raizen. Enhanced escape rate for Hg $254 \mathrm{~nm}$ resonance radiation in fluorescent lamps. Journal of Physics D: Applied Physics, 46(41):415204, 2013.

[8] M.J. Rivard, L.M. Bobek, R.A. Butler, M.A. Garland et al. The US national isotope program: current status and strategy for future success. Applied radiation and isotopes: including data, instrumentation and methods for use in agriculture, industry and medicine, 63(2):157, 2005. 
[9] R. Van Noorden. Radioisotopes: The medical testing crisis. Nature, 504(7479):202, 2013.

[10] A. Al-Nahhas, Z. Win, T. Szyszko, A. Singh et al. Gallium-68 pet: A new frontier in receptor cancer imaging. Anticancer Research, 27:4087, 2007.

[11] J.P. Norenberg. The Impact of the Ge-68/Ga-68 on Molecular Imaging. MolecularImaging, Winter 2013.

[12] J.M. Connett, C.J. Anderson, L.W. Guo, S.W. Schwarz et al. Radioimmunotherapy with a ${ }^{64} \mathrm{Cu}$-labeled monoclonal antibody: a comparison with ${ }^{67} \mathrm{Cu}$. Proceedings of the National Academy of Sciences, 93(13):6814, 1996.

[13] S.M. Bilenky and C. Giunti. Neutrinoless Double-Beta Decay: A Brief Review. Modern Physics Letters A, 27(13):1230015, 2012.

[14] V. Lozza. Neutrinoless double beta decay search with SNO+. EPJ Web of Conferences, 65:01003, 2014.

[15] B. Andreas, Y. Azuma, G. Bartl, P. Becker et al. Counting the atoms in a ${ }^{28} \mathrm{Si}$ crystal for a new kilogram definition. Metrologia, 48:S1, 2011.

[16] B. Andreas, Y. Azuma, G. Bartl, P. Becker et al. Determination of the Avogadro Constant by Counting the Atoms in a ${ }^{28} \mathrm{Si}$ Crystal. Physical Review Letters, 106:030801, 2011.

[17] E. Lawrence and M. Livingston. The Production of High Speed Light Ions Without the Use of High Voltages. Physical Review, 40:19, 1932.

[18] A.L. Yergey and A.K. Yergey. Preparative scale mass spectrometry: A brief history of the calutron. Journal of the American Society for Mass Spectrometry, 8(9):943, 1997.

[19] L.O. Love. Electromagnetic Separation of Isotopes at Oak Ridge: An informal account of history, techniques, and accomplishments. Science, 182:343, 1973. 
[20] E. Newman. The stable isotope enrichment program at Oak Ridge National Laboratory. In Separated Isotopes: Vital Tools for Science and Medicine. National Academy Press, 1982.

[21] W.A. Bell and J.G. Tracy. Stable isotope separation in calutrons: Forty years of production and distribution. Oak Ridge National Laboratory (ORNL/TM-10356), 1987.

[22] D. Brown and S. Harrison. Production Techniques of Stable Metal Isotopes: Current Status and Future Trends. In Isotope Production and Applications in the 21st Century. World Scientific Publishing Company, 2000.

[23] J.W. Terry. Alternative isotope enrichment processes. Oak Ridge National Laboratory (CONF-8309127-1), 1983.

[24] J. Norenberg, P. Staples, R. Atcher, R. Tribble et al. Workshop on the nation's need for isotopes: Present and future. U.S. Department of Energy (DOE/SC0107), 2008.

[25] J.A. Paisner. Atomic vapor laser isotope separation. Applied Physics B Photophysics and Laser Chemistry, 46:253, 1988.

[26] J. Dawson, H. Kim, D. Arnush, B. Fried et al. Isotope Separation in Plasmas by Use of Ion Cyclotron Resonance. Physical Review Letters, 37:1547, 1976.

[27] M.G. Raizen and B. Klappauf. Magnetically activated and guided isotope separation. New Journal of Physics, 14:023059, 2012.

[28] A. Kastler. Quelques suggestions concernant la production optique et la détection optique d'une inégalité de population des niveaux de quantifigation spatiale des atomes. Application à l'expérience de Stern et Gerlach et à la résonance magnétique. Journal de Physique et le Radium, 11:255, 1950.

[29] Z. Xiwen, H. Guilong, M. Ganghua, and Y. Delin. Laser isotope enrichment of lithium by magnetic deflection of a polarized atomic beam. Journal of Physics B: Atomic, Molecular and Optical Physics, 25:3307, 1992. 
[30] W. van Wijngaarden and J. Li. Laser isotope separation of barium using an inhomogeneous magnetic field. Physical Review A, 49:1158, 1994.

[31] H.R. Noh, J.O. Kim, D.S. Nam, and W. Jhe. Isotope separation in a magnetooptical trap. Review of Scientific Instruments, 67:1431, 1996.

[32] K. Kok, editor. Nuclear Engineering Handbook. Mechanical Engineering Series. CRC Press, 2009.

[33] D. LeBlanc. Molten salt reactors: A new beginning for an old idea. Nuclear Engineering and Design, 240:1644, 2010.

[34] Managing Critical Isotopes: Stewardship of Lithium-7 Is Needed to Ensure a Stable Supply. United States Government Accountability Office (http://www.gao.gov/products/gao13-716), 2013.

[35] G.M. Bruce, S.M. Flack, T.R. Mongan, and T.E. Widner. Mercury Releases from Lithium Enrichment at the Oak Ridge Y-12 Plant - a Reconstruction of Historical Releases and Off-Site Doses and Health Risks. Tennessee Department of Health (https://health.state.tn.us/ceds/OakRidge/Mercury1.pdf), 1999.

[36] E.W. Rothe, B.P. Mathur, and G.P. Reck. Isotope fractionation in two-step photoionization of $\mathrm{Li}_{2}$. Chemical Physics Letters, 53:74, 1978.

[37] T. Arisawa, Y. Maruyama, Y. Suzuki, and K. Shiba. Lithium isotope separation by laser. Applied Physics B Photophysics and Laser Chemistry, 28:73, 1982.

[38] I.E. Olivares, A.E. Duarte, E.A. Saravia, and F.J. Duarte. Lithium Isotope Separation With Tunable Diode Lasers. Applied Optics, 41:2973, 2002.

[39] M. Saleem, S. Hussain, M. Rafiq, and M.A. Baig. Laser isotope separation of lithium by two-step photoionization. Journal of Applied Physics, 100:053111, 2006.

[40] W.M. Haynes, editor. CRC Handbook of Chemistry and Physics. CRC Press, 2014. 
[41] G. Noble, B. Schultz, H. Ming, and W. van Wijngaarden. Isotope shifts and fine structures of ${ }^{6,7} \mathrm{Li} \mathrm{D}$ lines and determination of the relative nuclear charge radius. Physical Review A, 74:012502, 2006.

[42] E. Arimondo, M. Inguscio, and P. Violino. Experimental determinations of the hyperfine structure in the alkali atoms. Reviews of Modern Physics, 49:31, 1977.

[43] A. Corney. Atomic and Laser Spectroscopy. Clarendon Press, 1977.

[44] G. Breit and I. Rabi. Measurement of Nuclear Spin. Physical Review, 38:2082, 1931.

[45] H.A. Bethe and E.E. Salpeter. Quantum Mechanics of One- and Two-electron Atoms. Dover Publications, 2008.

[46] D. Budker, D. Kimball, and D. DeMille. Atomic physics: An exploration through problems and solutions. Oxford University Press, 2008.

[47] K. Viering. Experiments to Control Atom Number and Phase-Space Density in Cold Gases. PhD thesis, The University of Texas at Austin, 2012.

[48] D. Medellin. Towards the creation of high-fidelity Fock states of neutral atoms. PhD thesis, The University of Texas at Austin, 2013.

[49] P.W. Milonni and J.H. Eberly. Lasers. Wiley, 1988.

[50] W. McAlexander, E. Abraham, and R. Hulet. Radiative lifetime of the $2 \mathrm{P}$ state of lithium. Physical Review A, 54:R5, 1996.

[51] H.J. Metcalf and P. Straten. Laser Cooling and Trapping. Springer, New York, NY, 1999.

[52] W. Happer. Optical Pumping. Reviews of Modern Physics, 44:169, 1972.

[53] P. Tremblay and C. Jacques. Optical pumping with two finite linewidth lasers. Physical Review A, 41:4989, 1990. 
[54] G. Baum, C.D. Caldwell, and W. Schröder. Dual-frequency optical pumping for spin-polarizing a lithium atomic beam. Applied Physics, 21:121, 1980.

[55] J. Gillot, A. Gauguet, M. Büchner, and J. Vigué. Optical pumping of a lithium atomic beam for atom interferometry. The European Physical Journal D, 67:263, 2013.

[56] D. Peterson and L. Anderson. Effect of radiation trapping on the polarization of an optically pumped alkali-metal atomic beam. Physical Review A, 43:4883, 1991.

[57] G. Brown, K. Halbach, J. Harris, and H. Winick. Wiggler and undulator magnets - A review. Nuclear Instruments and Methods in Physics Research, 208:65, 1983.

[58] J. Mallinson. One-sided fluxes - A magnetic curiosity? IEEE Transactions on Magnetics, 9:678, 1973.

[59] K. Halbach. Design of permanent multipole magnets with oriented rare earth cobalt material. Nuclear Instruments and Methods, 169:1, 1980.

[60] M. Jerkins, I. Chavez, U. Even, and M.G. Raizen. Efficient isotope separation by single-photon atomic sorting. Physical Review A, 82:033414, 2010.

[61] M. Jerkins. From Atoms to Astronomy: New Approaches in Neutrino Physics. PhD thesis, The University of Texas at Austin, 2010.

[62] U. Even, J. Jortner, D. Noy, N. Lavie et al. Cooling of large molecules below $1 \mathrm{~K}$ and He clusters formation. The Journal of Chemical Physics, 112:8068, 2000.

[63] B. Ghaffari, J. Gerton, W. McAlexander, K. Strecker et al. Laser-free slow atom source. Physical Review A, 60:3878, 1999.

[64] J.P. Beardmore, A.J. Palmer, K.C. Kuiper, and R.T. Sang. A hexapole magnetic guide for neutral atomic beams. The Review of scientific instruments, 80:073105, 2009.

[65] L.V. Hau, J.A. Golovchenko, and M.M. Burns. A new atomic beam source: The "candlestick". Review of Scientific Instruments, 65:3746, 1994. 
[66] C.A. Stan and W. Ketterle. Multiple species atom source for laser-cooling experiments. Review of Scientific Instruments, 76:063113, 2005.

[67] D. Raimi-Zlatic. Storing and Focusing Atomic Hydrogen to Measure the Rate of Reaction of Three Body Association. Undergraduate thesis, The University of Texas at Austin, 2014.

[68] G.C. Bjorklund, M.D. Levenson, W. Lenth, and C. Ortiz. Frequency modulation (FM) spectroscopy. Applied Physics B Photophysics and Laser Chemistry, 32:145, 1983.

[69] U. Schünemann, H. Engler, R. Grimm, M. Weidemüller et al. Simple scheme for tunable frequency offset locking of two lasers. Review of Scientific Instruments, $70: 242,1999$.

[70] D.A. Steck. Quantum Chaos, Transport, and Decoherence in Atom Optics. PhD thesis, The University of Texas at Austin, 2001.

[71] K.B. MacAdam. A narrow-band tunable diode laser system with grating feedback, and a saturated absorption spectrometer for Cs and Rb. American Journal of Physics, 60:1098, 1992.

[72] J.H. Moore, C.C. Davis, M.A. Coplan, and S.C. Greer. Building Scientific Apparatus. Cambridge University Press, 2009.

[73] T.P. Meyrath. Experiments with Bose-Einstein Condensation in an Optical Box. PhD thesis, The University of Texas at Austin, 2005.

[74] B. Anderson and M. Kasevich. Loading a vapor-cell magneto-optic trap using light-induced atom desorption. Physical Review A, 63:023404, 2001.

[75] E Rasel, F. Pereira Dos Santos, F. Saverio Pavone, F. Perales et al. White light transverse cooling of a helium beam. The European Physical Journal D, 7:311, 1999. 
[76] H. Ishimaru. Fast pump-down aluminum ultrahigh vacuum system. Journal of Vacuum Science 8 Technology A: Vacuum, Surfaces, and Films, 10:547, 1992.

[77] J.R. Chen. Thermal outgassing from aluminum alloy vacuum chambers. Journal of Vacuum Science \& Technology A: Vacuum, Surfaces, and Films, 3:2188, 1985.

[78] R.E. March and R.J. Hughes. Quadrupole Storage Mass Spectrometry. Wiley Interscience, New York, 1989.

[79] R. Delhuille, A. Miffre, E. Lavallette, M. Buchner et al. Optimization of a Langmuir-Taylor detector for lithium. Review of Scientific Instruments, 73:2249, 2002.

[80] M.J. Watts. A tensioning spring for high temperature ribbon filaments. Journal of Physics E: Scientific Instruments, 5:1044, 1972.

[81] A.A. Libson. General Methods of Controlling Atomic Motion: Experiments with Supersonic Beams as a Source of Cold Atoms. PhD thesis, The University of Texas Austin, 2012.

[82] R. Cote, M.J. Jamieson, Z.C. Yan, N. Geum et al. Enhanced cooling of hydrogen atoms by lithium atoms. Physical Review Letters, 84:2806, 2000.

[83] R. DeCarvalho, N. Brahms, B. Newman, J.M. Doyle et al. A new path to ultracold hydrogen. Canadian Journal of Physics, 83(4):293, 2005.

[84] C.-S. Lu. Investigation of film-thickness determination by oscillating quartz resonators with large mass load. Journal of Applied Physics, 43:4385, 1972.

[85] V.M. Mecea. Is quartz crystal microbalance really a mass sensor? Sensors and Actuators A: Physical, 128:270, 2006.

[86] H. Pauly. Atom, Molecule, and Cluster Beams I. Springer-Verlag, Berlin, 2000.

[87] K.J. Ross and B. Sonntag. High temperature metal atom beam sources. Review of Scientific Instruments, 66:4409, 1995. 
[88] F. Dumas-Bouchiat, L.F. Zanini, M. Kustov, N.M. Dempsey et al. Thermomagnetically patterned micromagnets. Applied Physics Letters, 96:102511, 2010.

[89] M. Kustov, P. Laczkowski, D. Hykel, K. Hasselbach et al. Magnetic characterization of micropatterned Nd-Fe-B hard magnetic films using scanning Hall probe microscopy. Journal of Applied Physics, 108:063914, 2010.

[90] J.L. Chilla, S.D. Butterworth, A. Zeitschel, J.P. Charles et al. High-power optically pumped semiconductor lasers. In Proceedings of SPIE, volume 5332, page 143. SPIE, 2004.

[91] V. Ostroumov, C. Simon, H. Schwarze, R. von Elm et al. 1 W 488 nm cw aircooled optically pumped semiconductor laser. In Proceedings of SPIE, volume 6871, page 687118. SPIE, 2008.

[92] Y. Kaneda, J.M. Yarborough, L. Li, N. Peyghambarian et al. Continuous-wave allsolid-state $244 \mathrm{~nm}$ deep-ultraviolet laser source by fourth-harmonic generation of an optically pumped semiconductor laser using $\mathrm{CsLiB}_{6} \mathrm{O}_{10}$ in an external resonator. Optics Letters, 33:1705, 2008.

[93] J. Paul, Y. Kaneda, T.-L. Wang, C. Lytle et al. Doppler-free spectroscopy of mercury at $253.7 \mathrm{~nm}$ using a high-power, frequency-quadrupled, optically pumped external-cavity semiconductor laser. Optics Letters, 36:61, 2010.

[94] T. Loftus, J. Bochinski, and T. Mossberg. Magnetic trapping of ytterbium and the alkaline-earth metals. Physical Review A, 66:013411, 2002.

[95] A. Derevianko. Feasibility of Cooling and Trapping Metastable Alkaline-Earth Atoms. Physical Review Letters, 87:023002, 2001.

[96] A.A. Celikov, A.M. Akulshin, V.L. Velichansky, and A.S. Zibrov. Doppler-free spectroscopy of the $5^{1} \mathrm{~S}_{0}-5^{3} \mathrm{P}_{1}$ strontium intercombination transition. Laser Physics, 5:739, 1995. 
[97] U. Sterr, K. Sengstock, J.H. Müller, and W. Ertmer. High-resolution isotope shift measurement of the MgI ${ }^{1} \mathrm{~S}_{0}-{ }^{3} \mathrm{P}_{1}$ intercombination transition. Applied Physics B Photophysics and Laser Chemistry, 56:62, 1993.

[98] W. Williams, Z.-T. Lu, K. Rudinger, C.-Y. Xu et al. Spectroscopic study of the cycling transition $4 s[3 / 2]_{2}-4 p[5 / 2]_{3}$ at $811.8 \mathrm{~nm}$ in ${ }^{39} \mathrm{Ar}$ : Hyperfine structure and isotope shift. Physical Review A, 83:012512, 2011.

[99] R.S. Williamson III. Magneto-optical trapping of potassium isotopes. PhD thesis, University of Wisconsin - Madison, 1997.

[100] A.K. Mollema. Laser cooling, trapping and spectroscopy of calcium isotopes. PhD thesis, University of Groningen, 2008.

[101] C. Lorenzen, K. Niemax, and L. Pendrill. Isotope shifts of energy levels in the naturally abundant isotopes of strontium and calcium. Physical Review A, 28:2051, 1983.

[102] P.O. Schmidt. Scattering properties of ultra-cold chromium atoms. PhD thesis, University of Stuttgart, 2003.

[103] B. Furmann, A. Jarosz, D. Stefańska, J. Dembczyński et al. Isotope shift in chromium. Spectrochimica Acta Part B: Atomic Spectroscopy, 60:33, 2005.

[104] S. Krins, S. Oppel, N. Huet, J. von Zanthier et al. Isotope shifts and hyperfine structure of the Fe I 372-nm resonance line. Physical Review A, 80:062508, 2009.

[105] B. Smeets, R.W. Herfst, L.P. Maguire, E. Te Sligte et al. Laser collimation of an Fe atomic beam on a leaky transition. Applied Physics B, 80:833, 2005.

[106] A. Steudel, U. Triebe, and D. Wendlandt. Isotope shift in Ni I and changes in mean-square nuclear charge radii of the stable $\mathrm{Ni}$ isotopes. Zeitschrift für Physik A Atoms and Nuclei, 296:189, 1980.

[107] J.J. Kim and N. Sung. Stimulated emission in optically pumped atomic-copper vapor. Optics Letters, 12:885, 1987. 
[108] G.F. Hately and T.A. Littlefield. Isotope Shift in the Arc Spectrum of Zinc. Journal of the Optical Society of America, 48:851, 1958.

[109] O.M. Maragò, B. Fazio, P.G. Gucciardi, and E. Arimondo. Atomic gallium laser spectroscopy with violet/blue diode lasers. Applied Physics B: Lasers and Optics, $77: 809,2003$.

[110] S. Rehse, K. Bockel, and S. Lee. Laser collimation of an atomic gallium beam. Physical Review A, 69:063404, 2004.

[111] S.J. Rehse, W.M. Fairbank, Jr., and S.A. Lee. Measurement of the hyperfine structure of the $3 d^{2} D_{3 / 2,5 / 2}$ levels and isotope shifts of the $4 p^{2} \mathrm{P}_{3 / 2} \rightarrow 4 d^{2} \mathrm{D}_{3 / 2}$ and $4 p^{2} \mathrm{P}_{3 / 2} \rightarrow 4 d^{2} \mathrm{D}_{5 / 2}$ transitions in gallium 69 and 71. Journal of the Optical Society of America B, 18:855, 2001.

[112] C.Y. Chen. Ultrasensitive Isotope Trace Analyses with a Magneto-Optical Trap. Science, 286:1139, 1999.

[113] G.P. Barwood, P. Gill, and W.R.C. Rowley. Frequency measurements on optically narrowed Rb-stabilised laser diodes at $780 \mathrm{~nm}$ and $795 \mathrm{~nm}$. Applied Physics B Photophysics and Laser Chemistry, 53:142, 1991.

[114] X. Xu, T.H. Loftus, J.L. Hall, A. Gallagher et al. Cooling and trapping of atomic strontium. Journal of the Optical Society of America B, 20:968, 2003.

[115] R. Hughes. Isotope Shifts in the Spectra of Strontium. Physical Review, 105:1260, 1957.

[116] C. Hancox, M. Hummon, S. Nguyen, and J. Doyle. Evaporative cooling of magnetically trapped atomic molybdenum. Physical Review A, 71:031402, 2005.

[117] P. Aufmuth, H.-P. Clieves, K. Heilig, A. Steudel et al. Isotope shift in molybdenum. Zeitschrift für Physik A Atoms and Nuclei, 285:357, 1978.

[118] W. Whaling and J.W. Brault. Comprehensive transition probabilities in Mo I. Physica Scripta, 38:707, 1988. 
[119] G. Uhlenberg, J. Dirscherl, and H. Walther. Magneto-optical trapping of silver atoms. Physical Review A, 62:063404, 2000.

[120] R. Kloch, P.E.G. Baird, M.G. Boshier, M.J. Macpherson et al. Isotope shifts in $\lambda 326.1 \mathrm{~nm}$ of CdI. Zeitschrift für Physik D Atoms, Molecules and Clusters, 6:315, 1987.

[121] B. Klöter, C. Weber, D. Haubrich, D. Meschede et al. Laser cooling of an indium atomic beam enabled by magnetic fields. Physical Review A, 77:033402, 2008.

[122] C.-J. Lorenzen, K. Niemax, and K.-H. Weber. Level isotope shifts of indium 113 and 115. Optics Communications, 52:178, 1984.

[123] M. Walhout, H. Megens, A. Witte, and S. Rolston. Magneto-optical trapping of metastable xenon: Isotope-shift measurements. Physical Review A, 48:R879, 1993.

[124] S. De. Laser cooling and trapping of barium. PhD thesis, University of Groningen, 2008.

[125] V.N. Gorshkov, V.A. Komarovskii, A.L. Osherovich, and N.P. Penkin. Lifetimes of excited levels of Nd I and Nd II. Oscillator strengths of the spectral lines of Nd I. Astrophysics, 17:437, 1982.

[126] E.A. Den Hartog, A.J. Fittante, and J.E. Lawler. Radiative lifetimes of neutral neodymium. Journal of Physics B: Atomic, Molecular and Optical Physics, 44:225001, 2011.

[127] M.H. Stockett, M.P. Wood, E.A. Den Hartog, and J.E. Lawler. Atomic transition probabilities of Nd I. Journal of Physics B: Atomic, Molecular and Optical Physics, 44:235003, 2011.

[128] K. Blaum, B.A. Bushaw, S. Diel, Ch. Geppert et al. Isotope shifts and hyperfine structure in the transitions of gadolinium. The European Physical Journal D, 11:37, 2000. 
[129] N. Leefer, A. Cingöz, B. Gerber-Siff, A. Sharma et al. Transverse laser cooling of a thermal atomic beam of dysprosium. Physical Review A, 81:043427, 2010.

[130] N. Leefer, A. Cingöz, and D. Budker. Measurement of hyperfine structure and isotope shifts in the Dy $421 \mathrm{~nm}$ transition. Optics Letters, 34:2548, 2009.

[131] M. Lu, S.H. Youn, and B.L. Lev. Spectroscopy of a narrow-line laser-cooling transition in atomic dysprosium. Physical Review A, 83:012510, 2011.

[132] W.-G. Jin, H. Ono, and T. Minowa. Isotope Shifts in High Lying Levels of Dy I and Er I by High-Resolution UV Laser Spectroscopy. International Journal of Spectroscopy, 2011:1, 2011.

[133] J. McClelland and J. Hanssen. Laser Cooling without Repumping: A MagnetoOptical Trap for Erbium Atoms. Physical Review Letters, 96:143005, 2006.

[134] H.Y. Ban, M. Jacka, J. L. Hanssen, J. Reader et al. Laser cooling transitions in atomic erbium. Optics Express, 13:3185, 2005.

[135] R. Maruyama. Optical trapping of ytterbium atoms. PhD thesis, University of Washington, 2003.

[136] K. Pandey, A. Singh, P. Kumar, M. Suryanarayana et al. Isotope shifts and hyperfine structure in the 555.8-nm ${ }^{1} S_{0} \rightarrow{ }^{3} P_{1}$ line of Yb. Physical Review A, 80:022518, 2009.

[137] P. Villwock, S. Siol, and Th. Walther. Magneto-optical trapping of neutral mercury. The European Physical Journal D, 65:251, 2011.

[138] W.G. Schweitzer, Jr. Hyperfine Structure and Isotope Shifts in the 2537-A Line of Mercury. Journal of the Optical Society of America, 51:692, 1961.

[139] C.J. Schuler, M. Çiftan, L.C. Bradley, III, and H.H. Stroke. Hyperfine Structure and Isotope Shift in the $7^{2} \mathrm{~S}_{1 / 2}-6^{2} \mathrm{P}_{1 / 2}$ Transition of Natural Thallium by Atomic Beam Absorption. Journal of the Optical Society of America, 52:501, 1962. 
[140] I. Fan, T.-L. Chen, Y.-S. Liu, Y.-H. Lien et al. Prospects of laser cooling in atomic thallium. Physical Review A, 84:042504, 2011. 